

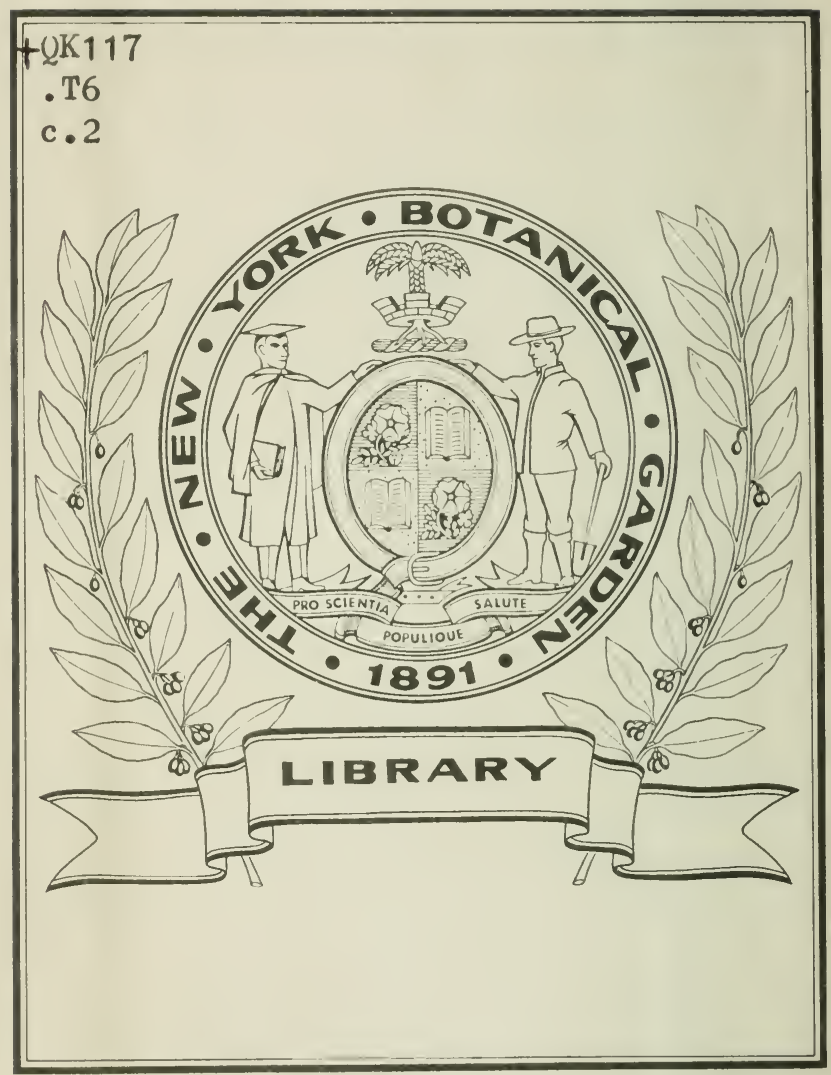








\section{GOMPENDIUM OF THE FLORA}

OF THE

\section{- Tortyerit and fetiarle states.}

IONTAINING GENERIC AND SPECIFIC, DESCRIPTIONS OF ALL TH PLANTS, EXCLUSIVE OF THE CRYPTOGANJA, HITHERTO FOUND IN THE UNITED STATES, NORTH OF THE POTONAR.

\section{BY JOHN TORREY, M.D.}

PROFESSOR OF CIENISTRY IN THE WEST-POINT MILITARY ACAIl FNY, PRESIDENT OF THE LYCEUAI OF NATURAL HISTORY

OF NEW-YORK, MEMBER OF THE PHYSIOGRAPHICAI. SOCIETY OF LUND, SWEDEN, OF THE WERNERIAN SOCIETY OF EDINBLRGH, \&C.

\section{VEW-YORK :}

STACY B. COLIINS, 65 FULTON-STRELT

I. \& I. Harper, Printerp

1826. 


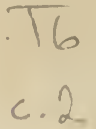

\section{SOUTHERN DISTHICT OF NEW-YURK, ss.}

BE IT REMEMBERED, that on the 16th day of September, in the fifty-first year of the Independence of the United States of Amer. ica, Stacy B. Collins, of the said District, hath deposited in this office the title of a book, the right where of he clains, as Froprietor, in the words following, to wit:

"A Compendium of the Flora of the Northern and Middle States. Containing Generic and Specific Descriptions of all the Plants, exclusire of the Cryptogamia, hitherto found in the United States, north of the Potomac. By John Torrey, M.D. Professor of Chemistry in the West-Point Military Academy, President of the Lyceum of Naturál History of New-York, Member of the Physiographical Society of Lund, Sweden, of the Wernerian Socicty of Edinburgb, \&c."

In conformity to the Act of the Congress of the United States, entitled "An Act for the encouragement of Learning, by securing the copies of Maps, Charts, and Books, to the authors and proprietors of such copies, during the times therein mentioned." And also to an Act, entitled, "An Act, supplementary to an Act, entitled an Act for the encouragement of Learning, by sccuring the cojies of Maps; charts, and Books, to the authors and proprietors of such copies, during the times therein mentioned, and extending the be nefits thereof in the arts of designing, engraving, and etching historicul and other prints."

JAMES DILI, 
'TO

WILLIAM COOPLR, ISSQ.

OF NEW-YORK.

\section{THIS VOLUME}

IS DEDICATED, AS A TESTIMONY

OF RESPECT FOR HIS

A'T'AINMENTS AS A NATURALIST,

$$
\text { AND } \triangle S
$$

AN EXPRESSION OF

HIGH ESTEEM FOK HIS VIRTUES, BY HIS FRIEND,

THE AUTHOR.

$-32433$ 



\section{ADVER'TISEMEN' .}

'I'HIs little Work, which was announced about two years since, in the Preface to the Author's Flora of the Northern and Middle States, is at length offered to the Public. His appointment to the Chair of Chemistry, in the Military Academy of WestPoint, shortly after the first volume of that work was published, unavoidably interrupted his botanical pursuits; but he has now resumed them with unabated zeal. He trusts that with his present means of research and accumulated materials, the remainder of the work will at least equal that which has already been published, and received with so much indulgence by our botanists. Considerable progress has been made in preparing it for the press, and it will be completed with all the speed that is consistent with its faithful execution.

This Compendium, which is after the model of Smith's Compendium Flora Britannica, it is hoped will be found useful to students of Botany in the Northern and Middle States, and also to those who have made greater progress in the science, as its small size will enable them to use it without inconvenience in their herborizations. It contains synoptical descriptions of all the plants described in 
the author's larger work, together with the hatitat, or place of growth of each plant, its time of flowering, duration, and such other of the more important properties as are not contained in the specific character. An Index of the principal synonyms with the names to which they correspond in this work, will be found at the end of the. volume.

West-Point, Sept. 1st, 1826. 


\section{NO'TICE.}

THE Abbreviations will probably be understood by most of those who will use this work. For the benefit of younger students, however, those which might not be readily known are here explained.

alt. alternate. in. inch. sol. solitary.

anth. anther. lob. lobe. wh. white.

Ger. berry. num. numerous. $\odot$. annual.

cal. calyx. leg. legume. o. biennial.

cor. corolla. pet. petal, 4 . perennial.

fl. Hower. per. peduncle. $h_{2}$. shrub or tree.

fi. fruit. sizt. small. \$. naturalized.

jt. ivot. tub. tubular. t. doubtful.

gri. green. sej. segment. 



\section{MONANDRIA.}

\section{MONOGYNIA.}

1. SALICORNIA. Cal. turbinate, entire, somewhat ventricose, succulent. Cor. 0. Stam. 1-2. Style bifid. Seed 1, covered by the inflated calyx.

2. HIPPURIS. Cal. entire, very small. Cor. 0. Style received into a groove of the anther. Stig. simple. Seed 1.

\section{DIGYNIA.}

3. CALLITRICHE. Cal. inferior 2-leaved. Pet. 0. Cap. 2-celled, 4 -seeded, compressed.

4. BLITUM. Cal. 3-cleft. Cor. 0. Seed 1, covered by the calyx, which becomes a berry.

\section{MONOGYNIA.}

1. SALICORNIA. Atriplices. Glasswort.

1. S. herbacea : annual; stem erect or branched; joints compressed, notched at the summits; spikes pedunculate ; calyx truncate.

HAB. Salt-marshes. Aug.-Sept. ๑. 6-10 in. high, fleshy, leafless; style very short.

2. S. ambigua: perennial, procumbent, branching: joints crescent-shaped, small ; spikes opposite and alternate ; calyx truncate.

HAB. Salt-marshes. Jul.-Aug. $\delta$ or Ћ. Procumbent and assurgent; anth. purplish-yellow.

2. HIPPURIS. Haloragec. Mare's tail.

H. vulgaris: leaves verticillate in eights, linear-lanceolate, acute.

IIAB. Ponds and lakes. Aug. 4. Erect, simple, 1-2 fi.; flowers axillary, sessile, minute. 


\section{DIGYNLA.}

3. CALLITRICHE. Haloragece. Water-Chickweei.

1. C. verna $\beta$. intermedia: upper leaves spathulateobovate, inferior ones lined, obtuse, and emarginate ; flowers polygamous; margin of the capsule obtuse. $\mathrm{HAB}$. Floating in shallow waters. Apr.-Aug. $\odot$. Up permost leaves stellate, 3-nerved; flowers axillury; superior ones stamenif., middle ones perfect, lowest ones pistilif.

9. C. autumnalis: leaves all linear, 1-nerved, truncate, or emarginate; capsule with an acute margin; flowers all perfect.

HAB. Floating. Aug.-Sept. $\odot$. Somerhat branched; superior leaves a little broader, and 3-nerved.

2. C. terrestris: procumbent, diffuse; leaves oblong, uniform, rather obtuse; capsule grooved on the margin.

HAB. On wet ground. Jun.-Aug. ๑. Creeping, small; leaves crowded; flowers polygamous; capsule very short, and broad.

\section{BLITUM. Atriplices.}

1. B. capitatum: procumbent; leaves triangular-hastate; heads alternate, in a terminal, leafless spike.

HAB. Fields and waste places. $\odot$. Jun. Stem branched, $1 \mathrm{ft}$.; heads round, sessile; flowers crowded, inconspicuous; calyx red when ripe.

9. B. virgatum: leaves triangular-hastate; hearls scattered, lateral.

HAB. In similar situations as No. 1. ๑. June. Heads all lateral; calyx red when ripe.

3. B. maritimum : calyx membranaceous ; clusters axillary, spiked, naked; leaves lanceolate, attenuate at each extremity, incisely toothed.

HAB. Salt marshes. Aug. ○. Erect, much branched, 1-2 ft.; leaves succulent; teeth fero; calyx not becoming red; seed lenticular, vertical.
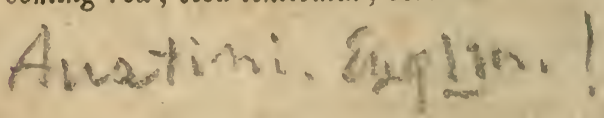


\section{DIANDRIA.}

1. MONOGYNIA.

* Flowers complete, inferior; 1 petalled.

$\uparrow$ Fruit a drupe or nut.

). LIGUSTRUM. Cal. 4-toothed. Cor. 4-cleft. Berry 2-celled; sells 2-seeded.

6. CHIONANTHUS. Cal. 4-parted. Cor. deeply 4 parted; segments long and linear. Drupe 1 -seeded.

Nut striate.

¿. ORNUS. Cal. 4-parted. Cor. 4-parted; petals lons and ligulate. Fil. 4. Nut winged. Fruit a capsule.

8. VERONICA. Cal. 4-parted. Cor. rotate, 4-lobed, unequal; the lower segrnent narrower. Caps. 2celled, obcordate; seeds few.

9. LEPTANDRA . Cal. 5-parted; segments acuminate. Cor. tubular-campanulate; border 4 -lobed, a little ringent; the lower segment narrower. Stam. and at length the pist. much exserted. Caps, ovate, acuminate, opening at the summit.

10. GRATIOLA. Cal. 5-parted, oflen with 2 bracts at the base. Cor. irregular, resupinate, 2-lipped; the upper lip 2 -lobed; the lower equally 3-cleft. Stig. 2-lipped. C'aps. 2-celled, 2-valved.

11. 1.INDERNIA. Cal. 5-parted. Cor. resupinate, tubular, 2-lipped; upper lip short, reflexed, emarginate; the lower lip trifid and unequal. Fil. 4 ; the two longer forked and sterile. Caps. 2-celled, 2-valved; dissepiment parallel with the valves.

12. HEMIAN'THUS. Cal. tubular, cleft on the under side; border 4 -toothed; upper lip of the cor. obsolete; lower 3-parted; intermediate segment ligulate, much longer, closely incurved. Stam. 2 ; fil. bifid; lateral fork antheriferous. Caps. 1-celled, 2-valved, many-seeded.

13. CATALPA. Cal. 2-parted. Cor. campanulate; tube ventricose; border 4-lobed, unequal. Stam. 2 fertile; $2-3$ sterile. Stig. bilamellate. Caps. long and cylindric, 2-valved; dissepiment opposite to the valves.

14. IUS'TICIA. Cal. 5-parted, often with 2 bracts at the 
base. Cor irregular, bilabiate ; upper lip emarginate; the lower 3-cleft. Anth. $1-2$ on each filament. Caps. attenuated, 2-celled, 2-valved ; dissepiment growing from the centre of each valve.

15. UTRICULARIA. Cal. 2-leaved, equal. Cor. personate or ringent; upper lip erect ; lower lip spurred at the base; palate subcordate. Fil. incurved. bearing the anthers within the apex. Stig. 2-lipped. Caps. 1-celled.

\section{$\dagger \dagger \dagger$ Seeds 4, naked.}

LABIATE.

16. LYCOPUS. Cal. tubular, 5-cleft, or 5-toothed. Cor. tubular, 4-cleft, nearly equal ; upper division broader and emarginate. Stam. distant. Seeds 4 . retuse.

17. CUNILA. Cal. cylindrical or tubular, 5-toothed, 10-striate. Cor. ringent; upper lip short, flat. Fertile stam. and style much exserted; sterile stam. very short.

18. HEDEOMA. Cal. 2-lipper, gibbous at the base ; upper lip 3-toothed; teeth lanceolate; lower lip of 2 subulate teeth. Cor. ringent. Stam. 2 fertile, as long as the corolla; 2 sterile, short.

19. MONARDA. Cal. 5-toothed, tubular. Cor. ringent; upper lip linear, involving the filaments; lower lip reflexed, 3-lobed.

20. SALVIA. Cal. subcampanulate, bilabiate; upper lip 2-3-toothed; lower lip bific. Cor. ringent. Fil. transversely affixed to a foot-stalk.

21. COLLINSONIA. Cal. bilabiate; upper lip 3toothed. Cor. unequal, somewhat campanulate, unequally 5-lobed; lower lobe divided irito many capillary segments. Seeds 4 ; three of them generally abortive.

\section{* Flowers superior.}

22. CIRCÆA. Cal. 2-leaved, superior. Cor. 2-petidled. Caps. 2-celled, not opening; cells 2 -seeded.

$$
\text { * * Flowers incomplete. }
$$

23. LEMNA. Cal. 1-leaved, entire. Cor. 0. Fruit an utricle. Seed 1, lying horizontally, and affixed by its lower side. 
24. CRYPTA. Cal. 2-leaved, inferior. Cor. 2-3petalled, closed. Style almost wanting. Stig. obtuse. Caps. 2-3-celled, 2-3-valved; cells 4-5seeded.

\section{MONOGYNIA.}

5. LIGUSTRUM. Prim, or Privet. Jasminece.

L. vulgare: leaves elliptical-lanceolate, smooth ; racemes compound, crowded.

HAB. Hedges and open woods. May. T. $6 \mathrm{ft}$. kigh; leaves opposite; flowers zehite, panicled; berries black.

6. CHIONANTHUS. Fringe-tree. Jasminece.

C. virginica: leaves terminal; peduncles 3 -flowered; leaves acute.

a. montana: leaves oval-lanceolate, coriaceous, smooth; panicles dense ; drupe oval.

ß. maritima: leaves obovate-lanceolate, membra. naceous, pubescent ; panicles very loose; drupe elliptical.

HAB. $\alpha$. On mountains ; $\beta$. on the sea-const. MayJune. h. 6-10-20 feet high; leav. oppos.; flowers in panicles.

7. ORNUS. Flowering-ash. Jasmincae.

O. americana: leaves broad-ovate, serrate; the terminal one obcordate.

JiAB. Shady woods. Z. $^{\mathrm{Fl}}$. as in Chionantuls; seeds small, riinged.

3. VERONICA. Speedwell. Rhinanthacexe.

1. V. officinalis: spikes lateral, peduncled; leaves obovate, or roundish, serrate, hairy ; stem procumbent.

HAB. Dry woods and meadows. June-July. 4 . Stern diffire; spikes erect, subradical; fl. blue.

$\therefore V$. serpyllifolia: raceme elongated, many-flowered; leaves ovate, crenate, smooth; capsule as long ae the style ; stem ascending. 
HAB. Meadows. May. 4. Stem procumbent at base, f. pale blue.

3. V. Beccabunga; racemes opposite ; leaves elliptical, obtuse, on short petioles, subserrate, glabrous ; stem procumbent, rooting at the base.

HAB. In water; rare. June. $4.1 \mathrm{ft}$. high, terete; racemes axillary, many-flow.; corol. blue; caps. inflat.

1. V. Anagallis: racemes opposite; leaves lanceolate, serrate; stem erect.

HAB. In water. June-Aug. 2 . $1 \frac{1}{2} \mathrm{ft}$. high, subgradrang.; peduncl. subpubescent; $f$. pale blue.

5. V. scutellata : racemes lateral and alternate ; (rarely opposite) pedicels divaricate ; leaves linear, somewhat toothed; stem nearly erect.

HAB. In water. May-June. 24. Root creeping; stem weak, somerohat branched; $f$. pale blue, or flesh-col.

6. V. arvensis: flowers solitary, subsessile; inferior leaves petiolate, cordate, ovate, serrate; stem leaves crenate; floral ones lanceolate, sessile, longer than the pedicels; segments of the calyx unequal ; capsule obcordate, compressed.

HAB. Dry hills and fields. Apr.-Aug. ๑. Stem assurgent, 2-6 in. high; $f$. pale blue; caps. compres.

7. V. agrestis: flowers solitary, pedicellate, leaves petiolate, cordate-ovate, serrate, segments of the calyx equal, ovate, hairy; stem procumbent, pubescent HAB. Sandy fields. May. ○. Branched, diffuse; fl. blue, veined; capsule didymous, subventricose.

3. V. peregrina: flowers solitary, sessile; leaves oblong. serrate, rather obtuse; stem erect.

HAB. Wet, clayey soils. May-July. $\odot$. Smooth; stem branched at the base; rad. leaves subpetiolate; $f l$. small, white; caps. obcordate.

7. V.hederifolia: flowers solitary ; leaves as long as the petioles, roundish-cordate, 5-lobed; the upper ones 3-lobed; segments of the calyx cordate, ciliate, acute; stem procumbent.

HAB. Shady rocks. A pril. $\odot$. Sterns diffuse, pubescent; leaves somerohat fleshy; caps. ventricose, with two seeds in each cell.

10. V. reniformis: stem procumbent, hispid; leaves ses- 
sile, reniform, entire, hairy, villous ; flowers axillary, solitary, subsessile.

HAB. Sandy soils. Summer. $\odot$. Stems few, terete, vitlous; fl. zehite or pale blue.

\section{LEPTANDRA. Antirrhinece.}

L. virginica: leaves verticillate, in fours or fives, lanceolate, serrate, petiolate.

HAB. Woods and fertile valleys. July-Aug. 24. Stem 3-4 ft. high; $f$. white, in a long terminal spike.

10. GRATIOLA. Hedge-hyssop. Scrophularic.

1. G. aurea: smooth ; leaves linear-oblong; half embracing the stem, obscurely toothed; leaves of the calyx equal; sterile filaments minute.

$\mathrm{HAB}$. Wet, sandy places, and in woods. Aug.-Sept. 4. Root creeping; stem 8-12 is.; fl. yellow.

9. G. virginica : stem pubescent, assurgent, terete; leaves smooth, lanceolate, sparingly dentate-serrate, alternate and connate at the base; leaves of the calyx equal; sterile filaments wanting.

HAB. Wet meadows. July-Aug. 24. 6-8 in., branched at the base; leaves smooth; cor. white; tube yellore.

3. G. megalocarpa: leaves lanceolate, serrate, pubescent; peduncles opposite, longer than the leaves; scgments of the calyx linear, as long as the globose capsule.

$H A B$. Ditches and pools. July-Aug. 4. Cor. paie yellow; caps. large.

11. LINDERNIA. Scrophularic.

1. L. dilatata: leaves dilated at the base, amplexicaul, remotely toothed; peduncles longer than the leaves. IIAB. Overflowed places. July-Sept. ๑. Stem assurgent; pedunc. altern. and oppos., spreading; cor. pale purple.

2. L. attenuata: leaves lanceolate and obovate, narrowed at the base; peduncles shorter than the leaves, erect.

HAB. Overflowed places. July-Sept. ๑. Stem erect or procumb. ; leaves serrate or dentate; cor. pale purple. 3. I. monticola: stem slender, dichotomous; radical 
leaves spathulate; cauline ones linear, small and renote; peduncles very long, at length reflexed.

$1 \mathrm{AB}$. On mountains. 4 . Leaves denticulate; $f$. pale blue.

\section{HEMIANTHUS. Lysimachice.}

11. micranthemoides.

IJ AB. Overflowed banks of rivers. Aug.-Sept ๑. Stem creeping; leaves oppos., crowded; fl. axill.; cor. bilab. tube gibbous; stam. inserted on the lower lip; style declined, bifid.

13. CATALPA. Catalpa. Bignonice.

C. cordifolia : leaves simple, cordate, entire; flowers in panicles.

MAB. Fields and about houses. July. A large tree; leaves in threes; $f$. in large term. panic.; caps. linear, slender; seeds caudate.

\section{JUSTICIA. Acanthacea.}

I. pedunculosa: spikes axillary; peduncles elongated. alternate; flowers crowded; leares lanceolate.

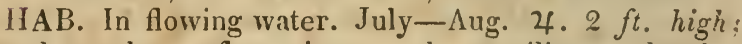
leaves long; fl. capitate, on long axillary peduncles. pale purp.; anth. 2, altern.

15. UTRICULARIA. Bladder-wort. Lentibularice.

1. U. inflata: floating; scape many flowered; radical leaves verticillate, inflated, pinnatifid at their extremities; lower lip of the corolla 3-lobed; spur deeply emarginate.

IIAB. Ponds. Aug. 4. Rad. leaves spongy; scape 5-8-fl.; cor. large, yellow.

?. U. vulgaris: floating; stems submerged, dichotomous ; leaves many-parted, vesiculose ; scape 5-9-flowered; upper lip of the corolla entire, broad-ovate: spur conical, incurved.

IIAB. In ponds of deep water. Aug. 24. Stems $1-3 \mathrm{ft}$.; leazes dichot.; fl. large, yellow; lower lip entire; spur entire.

3. U. minor: spur carinate; upper lip emarginate; palate equal : throat open; leaves 3-parted, dichotomous. 
HAB. Swamps and ditches. Aug. 2f. Stems decumbent; lower lip of the corol. obovate.

4. U. setacea: : scape filiform rooting, 2-3-flowered; upper lip of the corolla ovate; lower distinctly 3 lobed; spur sub-conical, shorter than the lower lip.

IIAB. Overflowed swamps. June. 24. Scap. 4-6 in., rooting in the mud; flowers mostly two, very small; spur rather obtuse.

5. U. gibba: floating; scape generally 2-flowered; spur shorter than the lower lip of the corolla, obtuse, gibbous in the middle.

IIAB. In ponds. July. 24. Roots dichot, with fow utric.; scape 2-3 in.; lips of the corol. roundish.

i. U. cornuta : scape rooting, erect, rigid; flowers $2-3$, subsessile ; inferior lip of the corolla very wide, 3lobed; spur very acute, porrected.

HAB. On wet calcareous rocks. Aug. - Sept. 4. Scape $1 \mathrm{ft}$., rooting in the mud, bracteolate; $f$. approx. large; palate very prominent.

i. U. striata: floating; scape 2-3-flowered; upper lip of the corolla ovate-round, sub-emarginate, waved on the margin; lower lip 3-lobed, reflexed at the sides ; spur straight, obtuse, shorter than the lower lip.

IIAB. In swamps. June. 24. Stems with ferw submerged branches; cor. yelloze, inuch expanded, striutc with red.

3. U. purpurea: scapes axillary, generally 1 -flowered; involucrum 0 ; lower lip of the corolla 3-lobed: lateral lobes cucullate on the under side; spur compressed, half as long as the corolla.

HAB. Ponds on mountains. Aug. 24. Stcms very long; scapes often by pairs; corolla purple!

16. LYCOPUS. Water Horehound. Labiate.

1. L. europeus $\beta$. angustifolius: smooth; stem acutely quadrangular; leaves narrow-lanceolate, with large acute teeth ; lower ones somewhat pinnatifid ; segments of the calyx acuminate, terminating in short spines.

HAB. Wet meadows and ditches. Aug. $\odot$. Root creeping ; stem 1-2 ft., much branched; leav. puncticul.: fl. verticill. crozeded, white.

จ. L. virginicus: leaves broad-lanceolite, serrate, nar- 
row and entire at the base; calyx shorter than the seed, spineless.

HAB. Shady, wet places. Aug. 24. Stem 1-1 $\frac{1}{2} f t$; nearly simple, with obtuse angles; leaves coarsely serrate, purplish beneath.

3. L. uniflorus: plant small ; root tuberous ; stem simple ; leaves oval, obtuse, obsoletely toothed; axils 1 -flowered.

HAB. - Aug. 24. Root tuberous; stolans leafy; stem. 2-3 in.; leaves petiolate.

\section{CUNILA. Labiutce.}

C. mariana: leaves ovate, sessile, serrate; corymbaxillary and terminal ; dichotomous.

HAB. On hills. Aug. - Sept. 4 Leav. oppos., punctute: cor. pink: stam. exserted.

\section{HEDEOMA. Wild Pennyroyal. Labiato.}

11. pulegioides: leaves oblong, remotely serrate: peduncles axillary, numerous.

HAB. Dry hills and woods. July-Aug. ๑. Plant aromatic; fl. subverticillate, pale blue.

\section{MONARDA. \\ * Culyx nearly equal.}

1. M. didyma: leaves ovate, acuminate, subcordate, somewhat hairy; flowers in simple or proliferous hearls; exterior bracts large, coloured, lanceolate. HAB. River banks. July-Aug. 4. Stem quadrang. soinewhat pubescent; flowers large, scarlet.

6. angustifolia: leaves ovate-lanceolate, acuminate, and with the stem, pubescent.

IIAB. Boggy woods. July. 24. Stem more slender; heads rarely proliferous; leaves often attenuate at the base.

2. M. oblongata: pubescent; heads simple; exterior bracts ovate; calyx short, with the throat bearded, teeth divaricate; stem obtuse-angled, hairy above ; leaves oblong-lanceolate, rounded at the base.

HAB. Rocky situations. July. 24. Stem 2-3 ft. much branched; flowers flesh-colour; bracts coloured.

3. M. clinopodia: smooth; heads small, simple, terminal; exterior bracts broad-ovate, acute, entire, almost smooth ; calyx short, ciliate ; corolla pubes- 
cent, slender; leaves ovate-oblong, acuminate, serrate, a little hairy; stem obtuse-angled, smooth. HAB. Drygravelly soil. July - Sept. 2f. Fl. yellow, with purple specks.

1. M. fistulosa: nearly smooth; heads simple or proliferous, leafy; exterior bracts oblong, acute, glabrous ; calyx long, bearded; corolla hirsute ; leaves ovate, acuminate, serrate; petioles long, ciliate; stem smooth, obtuse-angled.

HAB. Among rocks, on river banks. Aug. 4 . Stem 2 ft., branched; flowers small, in dense heads, purplish, sometimes proliferous.

5. M. punctata : somewhat smooth ; flowers verticillate; bracts lanceolate, coloured, longer than the whorl; leaves lanceolate-oblong, remotely serrate; stem obtuse-angled, white, downy.

HAB. In sandy fields. Sept. 24 . ô. Stem branched, $2-3$

$f t$; leaves punctate; $f$. yellow, dotted with brown.

$$
\text { * Calyx bilabiate. }
$$

6. M. hirsuta: whole plant very hairy; flowers small, verticillate; bracts attenuated into awns; upper teeth of the calyx very short, the others setiform; leaves ovate, on long petioles, serrate.

HAB. On mountains. August. 4. Much branched, and almost woolly; fl. pale blue, spotted with purple: upper lip very short.

\section{SALVIA.}

1. S. urticifolia: viscous and villous; leaves ovateoblong, toothed, narrowed into a petiole at the base : calyx 3-cleft; the upper division 3-toothed.

HAB. On mountains. June. 4 . Leaves punctate beneath : spikes naked; whorls remote; fl. blue.

3. S. lyrata: radical leaves lyrate-sinuate; stem nearly leafless, retrorsely hairy; upper lip of the corolli: very short.

IIAB. Fields and borders of woods. June. 4. Stem a foot high, very hairy; whorls about 6-fl.; cor. blue.

3. S. Claytoni: leaves cordate-ovate, sinuate, toothed, rugose ; teeth of the upper lip of the calyx conni vent.

HAB.—June-Oct. $\delta$ and 4 . Fl. violet. 
21. COLLINSONIA. Horse-weed. Labialce.

C. canadensis : leaves broad-cordate, ovate, glabrous ; teeth of the calyx short, subulate; panicle terminal, compound.

HAB. Among rocks, in rich soil. Aug. 24. Smooth; leaves on long or short foot-stalks; fl. dull yellore.

22. CIRC艮A. Enchanter's nightshade. Onagrce.

1. C. lutetiana $\beta$. canadensis : stem erect ; leaves ovate. remotely toothed, opaque, nearly smooth.

HAB. Shady woods. July-Aug. 24. Stem 1-2 feet high, simple; $f$. in racemes, pedicellate, reddishrehite; fruit hispid.

2. C. alpina: stem branched, very smooth, often procumbent; leaves broad-cordate, membranaceous, acutely toothed, shining.

HAB. On barks of trees and wet mossy rocks. Aug. 2f. Very smooth, 6-8 in high; leaves very broad, subdiaphanous; fruit pubescent.

23. LEMNA. Duck's-meat. Naiades.

1. L. trisulca: fronds thin, elliptical-lanceolate, caudate at one extremity, at the other serrate, ; roots solitary. HAB. Pure stagnant waters. $\odot$. Fronds laterally proliferous, and appearing cruciate; root a solitary fibre, caiyptrate at the extremity.

2. L. minor: fronds nearly ovate, compressed; roots sulitary.

HAB. Stagnant waters. $\odot$. Fronds a line or a line and a half long, succulent, aggregated.

3. L. gibba: fronds obovate, nearly plane above, hemispherical beneath; roots solitary.

HAB. Stagnant waters. $\odot$. Fronds as large as the preceding, gibbous, pellucid and reticulated beneath.

4. L. polyrrhiza : fronds obovate-roundish, compressed : roots numerous, fasciculate.

HAB. On stagnant waters, often with No. 2. ๑. 3-4 lines long, rarely proliferous.

C. minima.

\section{CRYPTA. Portulacece.}

$\mathrm{HAB}$. Borders of ponds, and banks of rivers. $\odot$ ? Aug: minute, Prostrate and creeping, with the branches assurgent; leaves oppos. cuneate; fl. minute, axill. 


\section{TRIANDRIA.}

\section{MONOGYNIA.}

* Flowers superior.

$\dagger$ Complete.

25. FEDIA. Cal. 1-leaved, 3-6-tonthed. Cor. monopetalous, equally 5-cleft. Caps. crowned with the persistent calyx, 3-celled, only one of the cells usually fertile.

\section{$\dagger \dagger$ Incomplete.}

26. IRIS. Cor. 6-parted; 3 of the segments reflexed, the others erect or connivent. Style short, or 0. Stig. 3, petaloid, covering the stamens. Caps. 3-celled, manyseeded.

27. LACHNANTHES. Cal. superior, petaloid, externally hirsute, deeply 6-parted, nearly equal. Style de. clining; stig. minutely 3-lobed. Caps. globose, 3 -celled; cells $3-6$-seeded.

$$
\begin{gathered}
\text { * Flowers inferior. } \\
\text { † Complete. }
\end{gathered}
$$

28. COMniEliNA. Cal. 3 leaved. Cor. 3-petalled. Stam. $6 ; 3-4$ sterile, and furnished with cruciform glands. Caps. 3-celled, 3-valved, one of the valves often abortive.

29. XYKIS. Fl. in a roundish or oblong head. Cal. glumaceous, cartilaginous, 3-valved. Cor. 3-petalled, equal. Stig. 3-cleft. Caps. 1-celled, 3-valved.

\section{$\dagger \dagger$ Incomplete.}

30. SCHOLLERA. Spath 1 -flowered, Anth. of 2 forms. Cur. tube very long; limb deeply 6-parted. Caps. 1-celled.

31. Heterant HeRA. Cal. a spath. Cor. tube long and slender; border 6-parted, equal. Anth. of 2 forms. 
Caps. 3-celled, many-seeded, opening at the angles dissep. contrary.

32. SISYRINCHIUM. Spath. 2-leaved. Cor. 6-petalled, flat, equal. Stam . cohering below. Stig. 3-cleft. Caps. 3-celled.

*** Flowers glumaceous.

33. KYLLINGIA. $F l$. distinct, disposed in a roundish, sessile, subimbricated spike. Cal. 2-valved, 1 -flowered. Cor. 2-valved, longer than the calyx.

34. SCIRPUS. Glum. 1-valved, 1-flowered, imbricated on all sides. Cor. 0.

35. SCHCENUS. Glum. fascicled into a spike, paleaceous; the inferior ones empty. Cor. 0. Style deciduous. Seed 1, (mostly naked at the base.)

36. RHYNCHOSPORA. Glum. fascicled into a spike; the inferior ones empty. Cor. 0 . Seed 1 , crowned with the persistent style; base surrounded with bristles.

37. MARISCUS. Fl. distinct, in a somewhat imbricated spike. Cal. 2-valved, unequal, 3-flowered. Cor. 1-valved. Style 3-cleft. Seed triquetrous.

38. DULICHIUM. Spikes somewhat racemose, axillary ; spikelets linear-lanceolate, rather compressed. Glum. distichous, sheathing. Cor. 0 . Style very long, bifid; base persistent. Seed with bristles at the base.

39. CYPERUS. Spikelets compressed, distinct. Glum. imbricated in two rows. Cor. 0. Style deciduous. Seed 1. naked.

10. ERIOPHORUM. Glum. 1-valved, imbricated on all sides into a spike. Cor. 0. Seed surrounded with long dense wool.

11. FUIRENA. Glum. awned, imbricated on all sides into a spike. Cor. 3-valved, petiloid; valves cordate, awned, unguiculate.

12. CENCHRUS. Involuc. laciniate, echinate, including about 2 spikelets. Cal. 2-ralved, 2 -flowered; the inferior fl. sterile, the other perfect. Cor. awnless. Style 2-cleft.

13. SPARTINA. $F \%$ in unilateral spikes, imbricated in 2 rows. Cal. 2-valved, compressed, unequal. Cor. 2-ralved, awnless, unequal. Nect. collateral. 
44. ORYZOPSIS. Cal. 1-flowered, 2-valved; valves membranaceous, nearly equal, loose, obuvate, awnless. Cor. 2-valved, coriaceous, cylindrical-ovate, hairy at the base; the inferior valve awned at tip. Nect. linear, elongated.

\section{DIGYNIA.}

\section{A. Flowers all perfect. \\ $\dagger$ Spikelets 1-firwered. \\ * Call. 0.}

15. LEERsia. Cal. 0. Cor. 2-valved, closed; valves compressed, boat-shaped. Nect. obovate, entire, collateral.

$$
\text { * Cal. 2-valved. }
$$

a. Cor. zeithout abortive rudiments at the base.

1. Glumes and corolla of dissimilar texture; the inferior valve involving the superior.

\section{a. Cor. unarmed.}

16. PASP ALUM. Fl. in unilateral spikes. Cal. 2-valved, membranaceous, equal, nearly orbicular. Corr. cartilaginous, of the size and form of the calyx. Stig. plumose, coloured. Nect. collateral.

17. MILIUM. Cal.2-valved, herbaceous. Cor. 2-valved, coriaceous, oblong. cincave, shorter than the calyx, awnless. Seed 2-horned. Nect. collateral.

ß. Cor. arined or brintled at the tip.

18. PIPTATHERU \%. Cal. membranaceous, longer than the corollit. Cor. Cartilaginous, elliptical ; inferior valve awned at the tip. Nect. ovate, entire. Seed coiterl.

19. STIPA. Cal. 2-valverl, membranaceous. Cor. 2valved, shorter th:n the calys, coriaceous, involute. subcylindric ; awn terminal, contorted near the base. Seed coated.

50. ARISTIDA. Cul. 2-valved, membranaceous, unequal. Cor. 2-valved, pedicellate, subcylindric ; in. 
ferior valve coriaceous, involute, 3-awned at the tip ; superior valve very minute, or obsolete. Nect. collateral.

2. Glumes and corolla of nearly similar texture, often carinate.

a. Panicle more or less spreading.

51. MUHLENBERGIA. Cal. very minute, 2-valved, truncate, unequal. Cor. 2-valved, hairy at the base; inferior valve terminating in a slender bristle.

52. TRICHODIUM. Cal. 2-valved; valves nearly equal, serrulate on the keel. Cor.1-valved, smaller than the calyx. Stig. nearly sessile.

53. AGROSTIS. Cal. 2-valved, 1-flowered, com. pressed, herbaceous. Cor. 2-valved, membranaceous, generally larger than the calyx, often hairy at the base. Nect. collateral. Seed coated.

54. CINNA. Cal. 2-valved, compressed, nearly equal. Cor. linear, compressed, shortly stipitate, naked at the base ; inferior valve enclosing the superior, with a short awn near the summit.

55. POLYPOGON. Cal. 2-valved, 1-flowered; valves nearly equal, terminating in a bristle. Cor. 2-valved, shorter than the calyx; the inferior valve terminating in a bristle.

56. TRICHOCHLOA. Cal. 2-valved, 1-flowered; glumes very minute. Cor. much larger than the calyx, 2-valved, naked at the base; inferior valve convolute at the base, terminating in a long awn not articulated.

5\%. ARUNDO. Cal. 2-valved, unequal, membranaceous, surrounded with hair at the base; inferior valve mucronate or slightly awned; superior valve sometimes with a pencil-form rudiment at the base.

3. Panicle more or less contracted into a spike.

j8. PSAMMA. Cal 2-valved, awnless. Cor. shorter than the calys, surrounded with hairs at the base. Nect. linear-lanceolate, longer than the seed. Style 3parted ; stig. 3.

59. CRYPSIS. Cal. valved, membranaceous, carinate. Cor. longer than the calyx, which it resembles in texture and form. Stam. 2-3. Nect. 0. 
v0. ALOPECURUS. Cal. 2-valved, equal; glum. generally connate at the base. Cor. 1-valved, utriculate, cleft on one side, awned below the middle. Styles often connate.

61. PHLEUM. Cal. 2-valved, much longer than the corolla; glumes equal, boat shaped, rostrate or mucronate. Cor. 2-valved, included in the calyx, awnless, truncate.

C. Cor. with 1 or 2 abortive rudiments of florets at the base.

1. Glumes and corolla of dissimilar texture.

6?. CYNODON. Spikes digitate and fascicled. Fl. unilateral, in a simple series. Cal. 2-leaved; glum. nearly equal, spreading, acute. Cor. 2-valved; sup. valve very narrow, surrounded by the inferior one, Rudiment minute, pedicellate. Nect. collateral.

63. GYMNOPOGON. Cal. 2-valved, carinate, nearly equal, longer than the flower; valves of the cor. nearly equal; the inferior with a straight bristle a little below the tip. Rudiment aristiform.

2. Glumes and corolla of nearly similar texture.

6i. PHALARIS. Cal. 1-flowered, 2-valved, nearly equal, membranaceous, gibbous on the back, carinate. Cor. 2-valved, coriaceous, hairy at the base, shorter than the calyx. Rudiments opposite, sessile, resembling valves. Nect. collateral.

65. ANTHOXANTHUM. Cal. 2-valved, 1-flowered. Cor. 2-valved, with 2 abortive, 1 -valved rudiments at the base; one of them awned from near the base, the other from near the tip. Stam. 2.

1;6. BRACHYELY'TRUM. Cal. very minute; inferior glume scarcely perceptible. Cor. with the inferior valve terminated by a long bristle; superior valre. with a clavate rudiment it the base.

$\dagger \dagger$ Spikelets many-flowered.

* Florets all perfect.

a. Floziers panicled. 


\section{Corolla unarmed.}

67. PHRAGMITES. Cal. 5-7-flowered. Florets on villose pedicels, except the lowest, which is sessile and naked at the base; inferior valve elongated, acuminate and involute; superior valve somewhat conduplicate.

68. GLYCERIA. Spikelets terete, elongated. Cal. many-flowered, shorter than the florets; inferior valve of the corolla herbaceo-membranaceous ; superior valve somewhat conduplicate. Nect. collateral, connate. Stig. decompound.

39. UNIOLA. Spikelets compressed, ancipital, manyflowered. Cal.2-valved, shorter than the florets. Cor. 2-valved, awnless; inferior valve boat-shaped; superior valve smaller, concave on the back. Nect. collateral, emarginate.

70. BRIZA. Spikelets cordate-ovate, many-flowered. Cal. paleaceous, shorter than the distichous florets. Cor. ventricose; inferior valve cordate; superior nearly orbicular, very short.

71. POA. Spikelets oblong or linear, compressed, manyflowered. Cal. shorter than the florets. Cor. herbaceous, awnless, often arachnoid at the base: inferior valve scarious on the margin.

2. Corolla more or less setigerous or mucronate.

$$
\text { a. Seed free. }
$$

12. KCELERIA. Cal. 2-4-flowered, shorter than the florets. Inferior valve of the corolla mucronate, or with a short bristle a little below the tip.

73. URALEPIS. Cal. 2-3-flowered, much shorter than the florets, which are stipitate and distinct. Cor. Q-valved; valves very unequal, distinctly villose on the margin; the inferior tricuspidate; the central cusp produced into a short bristle; superior valve concave on the back, and incurved. Seed gibbous, coated.

74. TRICUSPIS. Spikelets terete,tumid, many-flowered. Calyx 2-valved, carinate, shorter than the florets. Inferior valve of the Cor. tricuspidate by the pro. jecting carina and margins; base and sides villous: superior valve emarginate. Nect. obtuse, entire. 


\section{ß. Seed adnate.}

75. FESTUCA. Spikelets often more or less terete, at length compressed, many-flowered. Cal. unequal, carinate, shorter than the florets. Cor. somewhat terete; superior valve acute, mucronate, or with a short bristle at the tip; superior valve bidentate.

76. CERATOCHLOA. Spikelets lanceolate, subancipital, many-flowered. Cal. shorter than the imbricated florets. Inferior valve of the corolla com. pressed, mucronate below the tip ; superior valve somewhat conduplicate. Style 2-3-parted.

77. DIARRHENA. Cal. 2-valved, very unequal, 2-5flowered, rigid; glumes acuminate, mucronate. Cor. cartilaginous; inferior valve entire, broader ; superior valve much shorter, emarginate. Seed coated, as long as the superior valve of the corolla. Nect. ovate, ciliate.

78. DACTYLIS. Spikelets aggregated in an unilateral head, many-flowered. Cal.shorter than the florets; one of the glumes larger, carinate and pointed. Inferior valve of the cor. carinate, emarginate, mucronate; superior valve somewhat conduplicate.

3. Corolla more or less bifid, armed between the divisions, a little below the tip.

79. DANTHONIA. Cal. 2-5-flowered, longer than the florets; glumes cuspidate. Cor. bearded at the base; inferior valve 2-toothed, with a twisted awn between the teeth; superior valve obtuse, entire.

P.0. TRISETUM. Cal. 3--5-flowered, membranaceous, as long as the florets. Inferior valve of the corolla with 2 bristle-form teeth at the tip, awned below the division of the teeth.

31. BROMUS. Spikelets oblong, distichous, many, nowered. C'al. shorter than the florets. Inferior valve of the corolla bifid, with a bristle between the teeth a little below the tip; superior valve subcon. duplicate, ciliate.

1. Inferior valve of the corolla azined on the back.

82. ARRHENATHERUM. Cal. membranaceous, 2. Howered, rather sborter than the florets. Inferior 
floret antheriferous; inferior valve 2-cleft, awned below the middle. Superior floret perfect; inferior valve cleft, unarmed, or with a short awn a little below the tip.

83. AVENA. Cal. membranaceous, many-flowered, generally longer than the florets. Cor. mostly bearded at the base : inferior valve bilaciniate, with a twisted awn on the back. Seed coated.

84. AIRA. Cal. 2-3-flowered, longer than the florets. Inferior valve of the Cor. eroded or many-toothed, awned on the back below the middle.

\section{b. Flowers spiked. \\ 1. Calyx 1-valved.}

35. LOLIUI. Spikelets sessile on a rachis, many-flowered. Calyx of 1 glume. Inferior valve of the cor. herbaceo-membranaceous, mucronate, or with a short bristle at the tip.

\section{Calyx 2-valved.}

36. ELEUSINE. Spikes digitate, unilateral. Cal. membranaceous, 4-6-flowered; glumes obtuse, unequal, Cor. membranaceous, unequal, unarmed; inferior valve carinate, the superior concave on the back. Seed triangular, transversely rugose.

87. TRITICUM. Spikelets sessile on the teeth of the rachis, 3-4-flowered; the 2 inferior florets opposite, fertile; the upper ones often abortire. Glum. broad, boat-shaped, mucronate or armed. Inferior valve of the cor.terminating in a bristle; superior ralve subconduplicate.

38. AGROPYRON. Spikelets sessile on the teeth of the rachis, 3-9-flowered. Glum. lanceolate, carinate. Inferior valve of the cor. mucronate, or terminating in a subulate bristle.

39. SECALE. Spikelets sessile on the teeth of the rachis, 2-3-flowered; the terminal floret abortive. Cal. 2-valved; glumes subulate, opposite, shorter than the florets. Inferior valve of the Cor. terminating in a long bristle.

90. ELYMUS. Spikelets 2 or more at each joint of the rachis, 3-9-lowered. Gbum.(involacrum) geminate, 
subulate. Inferior valve of the Cor. entire, mucro. nate, or with a short bristle at the tip.

* Terminal florets abortive, or mere rudiments.

91. MELICA. Cal.2-4-flowered,loose,membranaceous. Florets as long as the glumes; one or more of the superior ones abortive and incomplete. Cor. unarmed, coriaceo-membranaceous. Nect. connate. Seed free.

92. ATHEROPOGON. Spikes in an unilateral raceme. Cal.2-valved, membranaceous, 2-flowered; inferior glume setiform. Perfect floret 2-valved; inferior valve 3 -toothed, or 3-bristled; superior valve bifid. Abortive floret pedicellate, 2-valved, 3-bristled.

\section{B. Flowers polygamous. \\ $\dagger$ Panicled.}

93. PANICUM. Cal. 2-valved, 2-flowered; the inferior glume often very small. Florets dissimilar; the lower one abortive or antheriferous, 1 -2-valved; the inferior valve resembling in texture the glumes; superior membranaceous. Perfect floret with cartilaginous valves, unarmed.

34. HIEROCHLOA. Cal.2-valved, 3-flowered; glumes scarious. Lateral florets antheriferous, triandrous. Central floret perfect, diandrous, (rarely triandrous.) 95. HOLCUS. Cal. 2-flowered; glumes membranaceous, somewhat boat-shaped, mucronate. Florets polygamous; the inferior perfect, sessile, unarmed ; superior antheriferous or neuter, pedicellate, awned on the back near the middle.

$$
\begin{gathered}
\text { † Spiked. } \\
\text { * With an involucrum. }
\end{gathered}
$$

96. SETARIA. Spikelets with an involucrum of 2 or more bristles at the base. Cal.2-flowered; glumes unequal, herbaceous. Inferior floret abortive, 1-2. valved, herbaceous. Superior floret perfect, with cartilaginous glumes.

\section{* * Without an involucrum.}

97. DIGITARIA. Spikes linear, unilateral, generally 
digitate or fasciculate. Spikelets by pairs, on short bifid pedicels. Cal.1--2-valved, 2-flowered; inferior glume often inconspicuous. Inferior floret abortive, 1-valved, herbaceous. Superior floret perfect; valves coriaceous.

98. ANDROPOGON. Spikelets by pairs, polygamous; the inferior one abortive, (antheriferous or neuter,) on a bearded pedicel; glum. and cor. frequently very minute or wanting. Superior spikelet sessile. 1.flowered, perfect; glumes subcoriaceous; cor. 2-valved, shorter than the calyx, membranaceous ; the inferior valve generally awned.

99. HORDEUM. Spikelets 3 at each joint of the rachis, 1 -flowered, all perfect, or the lateral ones abortive. Glum. lateral, subulate.-Perfect $f$.: Cor. 2-valved; inferior valve terminating in a bris. tle. Seed coated.

\section{TRIGYNIA.}

100. HOLOSTEUM. Cal. 5-leaved, coloured within. Pet 5, 2-parted. Caps. 1-celled, bursting at the summit.

101. MOLLUGO. Cal. 5-leaved, coloured within. Cor. 0. Capsule 3-celled, 3-valved.

102. LECHE.A. Cal 3-leaved. Pet. 3. Style 0; stig. plumose. Caps.3-celled, 3-ralved, with as many interior valves opposite the others; cells 1 -seeded.

103. PROSERPINACA. Cal. superior, 3-parted, persistent. Cor. 0. Nut bony, triquetrous, 3-celled.

\section{TRIANDRIA.}

\section{MONOGYNIA.}

\section{FEDIA. Vuleriance.}

F. radiata: leaves spathulate-oblong, nearly entire; fruit pubescent, somewhat 4 -sided, naked at the top. HAB. In fields. May. ๑. Stem dichotomous, mostly erect; $f$. blue, in fastigzate corymbs. 


\section{IRIS. Flag. Flower-de-lace. Iridece.}

1. 1. versicolor: flowers beardless; stem terete, more or less flexuous; germen somewhat triangular; leaves ensiform.

«. sulcata: stem slightly compressed ; interior petals longer than the stigmas; germen with the angles distinctly sulcate; the sides concave; capsule oblong, ventricose ; angles indistinctly furrowed.

3. communis : stem erect, distinctly flexuous; leaves narrow-ensiform; interior petals a little shorter than the stigmas; angles of the germen not grooved when young; sides deeply concave ; capsule cylindrical-oblong.

HAB. In water. May-June. 4. Root large, creeping; 2-3 ft. high; $f$. b!ue, vnriegated with yell.

3. 1. virginica : flowers beardless; stem solid, terete; leaves very long and narrow; capsule elongated, prismatic, acute at each end.

IAB. Swamps. June. 24. Stem 3-5 ft. high, slender; leav. half an in. hroad; angles of the germ. deeply grooved.

3. I. lacustris : flowers without a bearded crest; leaves ensiform, much longer than the 1 -flowered scape ; petals nearly equal, attenuated on the tube; capsule turbinate, 3-sided, margined; roots tuberous.

HAB. Gravelly shores of likes. June. Leares $3-5 \mathrm{in}$. long; scape very short, compressed; pet. emarginate.

\section{LACHNANTHES. Homodoracex.}

L. tinctoria.

HAB. Sandy swamps, in pine barrens. July. 24. Root red, fibrous; stem 2-ft. high simple, woolly above; $f$. corymbose, woolly, yellow within.

\section{COMMELINA. Day-Hlower. Commelinece.}

11. C. angustifolia: assurgent, slender, rather smooth ; petals unequal, (one very minute ;) leaves linearlanceolate; bracts peduncled, short-cordate.

HAB. Borders of swamps. June. 4. Leaves sheathing. ciliate at the throat; bracts subfalcute; fl. blue; fcrile stam. 2.

3. Cirginica: petals nearly equal; stem erect, a little 
pubescent; leaves long-lanceolate, subpetiolate ; throat bearded.

HAB. Shady woods. July. 2f. Stem $2 \mathrm{ft}$. high; leaves sometimes ovate-lanceolate; fertile stam. 3.

29. XYRIS. Yellow-eyed grass. Juncece.

1. X. caroliniana: leaves linear, grass-like; scape ancipitous; head ovate, rather acute; scales round. HAB. Swamps, and wet sandy places. July-Aug. 4. Scape erect simple, $1 \mathrm{ft}$. high; $f$. yell. in a small scaly head.

9. X. brevifolia : leaves subulate-ensiform, short; head nearly globose; interior valves of the calyx shorter than the exterior, slightly notched.

HAB. Wet places. 4. Scape 12-18 in. high, com. pressed above; leaves twisted.

30. SCHOLLERA. Narcissi.

\section{S. graminea.}

HAB. In flowing water. July-Aug. 24. Floating; stem slender, dichotomous; leaves gramineous; $f$. small, yellowe, solitary; stam. 4 , one of them abortive.

31. HETERANTHERA. Narcissi.

H. reniformis: leaves orbicular-reniform; spath oblong, acuminate, 2-3-flowered.

HAB. Overflowed banks of rivers. July-Aug. 4 . Stem partly floating; leaves petiolate; fl. zohite, evanescent.

32. SISYRINCHIUM. Blue-eyed grass. Iridec.

1. S. anceps: scape ancipitous, winged, simple, nearly leafless ; spath about 4-flowered, unequal, shorter than the flowers; petals mucronate.

HAB. In wet meadows. May-June. 4. Stem slender: leaves grass-like; fl. small. blue; caps. globose.

S. S. mucronatum : scape simple, winged ; scape coloured; one of the valres extended into a long marcescent point.

HAB. In sandy fields. June-July. Cespitose: spath unequal, coloured: Al. blue. 


\section{KYLLINGIA. Cyperoidea.}

K. monocephala: stem filiform, 3-angled; head globose, sessile; involucrum 3-leaved, very long. HAB.- 4. Root creeping; clum 3-12 in. high; head solitary.

\section{SCIRPUS. Club-rush. Cyperoidece.}

- Seed surrounded with bristles at the base.

* Style articulated to the seed; base dilated and persistent. Seed often lenticular. (Spike solitary.)

1. S. tenuis : culm very slender, quadrangular; spike elliptical, acute at each end; glumes ovate, obtuse ; stamens 3 ; style 3-cleft; seed rugose.

HAB. Wet places. July-Aug. 4. Culm naked, 8-12 in. high; head brown; bristles 2-3, sometimes wanting.

2. S. glaucus: culm many-angled, glaucous; spike ovate, acute; glumes ovate, obtuse, or emarginate; stamens 3 ; style 2-cleft.

HAB. Borders of salt marshes. July. 4 . Culm slender, $1 \mathrm{ft}$. high; spike brozenish; bristles 4.

3. S. palustris: culm terete, inflated; spike oblong. lanceolate; glumes somewhat obtuse ; seed roundish, punctate and rugose; bristles scabrous.

HAB. Wet places. June. 4. Cespitose; culm 1-2 ft. high, sheathed at the base, leafless; seed crowned with a conical tubercle.

4. S. capitatus : stem terete, or somewhat compressed; spilse ovate, obtuse ; seed oval, compressed, smooth. HAB. Wet places. July-Aug. 24. Cespitose; culm attenuated below the spike; spike often globose; bris. tles 6.

7. S. acicularis : culm setaceous, quadrangular; spike ovate, acute, 3-6-flowered; glumes somewhat obtuse; stamens 3 ; style bifid; seed obovate.

HAB. Border of ponds. June-July. 24. Culm almost hair-like; inf. glume empty, large; seed striate; bristles 4.

f. S. pusillus: culm compressed and a little angular; spike ovate, compressed; seed obovate; stamens 3 ; style $2-3$-cleft. 
HAB. In salt marshes. July-Aug. 4. An inch high, growing in patches, like moss.

i. S. intermedius : culms cespitose, quadrangular, sulcate; spike ovate-oblong, acute; glumes rather acute; stamens 3 ; style 2 -cleft; seed broad-obovate, compressed; tubercle distinct.

HAB. Muddy banks of rivers. Sept. 4 . Ascending, 3-4 in. high; bristles 6 ; tubercle very minute.

8. S. planifolius: culm triquetrous; radical leaves flat; nearly equalling the culn; spike terminal, oblong, compressed, shorter than the cuspidate bracts at the base.

HAB. Bogs and wet woods. May-June. 4. Cespitose; leaves carinate; spike 6-fl. yellowish; inf. glumes very large, bracteiform.

9. S. subterminalis : culm floating, sulcate, inflated, leafy below; spike solitary, somewhat terminal, lanceolate; style 2-cleft; seed triquetrous.

IIAB. Ponds and rivulets. Aug. 4. Whole plant, except the spike, under water; culm, when dry, much roughened by the contracting medulla; leaves channelled.

10. S. cespitosus, $\beta$. callosus: culm cespitose, terete ; spike ovate, few-flowered; glumes thickened and cartilaginous at the tips; the inferior ones bracteiform, as long as the spike; sheaths with rudiments of leares.

HAB. On high mountains. July. 4. A span high; culm reith imbricated sheaths at the base; spikes yellowish ; bristles 6 .

\section{$\dagger \uparrow$ Style filiform, not bearded, deciduous.}

11. S. americanus: culm nearly naked, triquetrous; sides concave; spikes lateral, $(1-5$,) ovate, conglomerate, sessile; glumes round-ovate, mucronate; seed triquetrous, acuminate.

$\mathrm{HAB}$. In salt marshes, and on the banks of rivers. July-Aug. 4. Culın 3-5 feet high, sometimes leafy at base.

12. S. debilis: culms cespitose, deeply striate; spikes about 3, lateral, ovate, sessile; glumes ovate, obtuse, mucronate. 
HAB. Borders of ponds. Aug.-Sept. 24. A foot high; leaves ferw, subulate; spikes turgid; seed obovate; bristles $4-5$.

13. S. lacustris: culm terete, attenuated above, naked; panicle subterminal; spikes peduncled, ovate.

HAB. Ponds and river marshes. June. 24. Culm 4-8 ft. high, sheathed at the base; spike subpanicled; glum. ovate, subpubescent; seed obovate.

14. S. acutus: culm terete, (not attenuated,) spotted; peduncles numerous, sublateral; spikes oblong; glumes pubescent, mucronate.

HAB. Ponds and wet swamps. June-July. 4. Culm 4 ft. high, with oblong brown spots; panicle proliferous; glum. dilated, carinate.

15. S. atrovirens: culm triangular, leafy ; cyme terminal, compound, proliferous; involucrum 3-leaved; spikes conglomerate, ovate, acute; glumes ovate, mucronate, pubescent.

HAB. Wet meadows. June-July. 4. Culm obtusely triang. 2 ft.high; involuc. long; spikes dark green, in heads of 10-12.

16. S. brunneus : culn leafy, obtusely triangular; cyme decompound; involucrum 3-4-leaved; spike round-ovate, clustered in about sixes; glumes ovate, obtuse.

HAB. In water. Aug.-Sept. 4. Culm 2-3 ft. high; leaves long; spike brozen; seed longer than the bristles.

17. S. macrostachyos: culm triquetrous, leafy ; corymb clustered; involucruin about 3 -leaved, very long ; spikes oblong; glumes ovate. 3-cleft ; the middle segment subulate and reflexed; style 3-cleft.

HAB. In salt marshes and ditches. July-Aug. 4 . Culm 3-4 ft. high; spikes 6-10, very large.

$1 \dagger \uparrow$ Style filiform, deciduous. Bristles much longer than the seed.

18. S. Eriophorum: culm obtusely triangular, leafy; panicle decompound, proliferous, nodding; spikes all pedunculate; bristles surrounding the seed exserted.

IIAB. Swamps. Aug. 4. Culm 4-5 ft. high ; in- 
voluc. leafy, very long; panic. much divided; spikes small, ovate, woolly when ripe.

19. S. lineatus : culm triquetrous, leafy ; panicle terminal and lateral, decompound, at length nodding; involucrum 1-2-leaved; spikes ovate; glumes lanceolate, somewhat carinate; bristles longer than the seed.

HAB. Swamps. Aug. 24. Culm 2-3 ft. high; leares subdistichous; pan. at first erect; bristles inter. roven.

* Seed naked at the base.

† Style simple at the base, not articulated to the seed, deciduous.

20. S. subsquarrosus: culm triquetrous, nearly naked; incurved ; spikes 3, nearly terminal, ovate; glumes somewhat squarrose at the tip; involucrum 2. leaved, very long, unequal:

HAB. Banks of rivers. Aug. ○ ? Cespitose, about 2 in.high, almost setaceous; spikes turgid, crowded; glum. ovate, carinate.

31. S. capillaris: culm nearly naked, triquetrous, ca= pillary; spikes ovate; $2-3$ of them pedunculate, with an intermediate sessile one.

HAB. Sandy fields. Aug. $\odot ?$ ? span high, cespitose; leaves setaceous, short, serrulate; spikes brownish, subumbellate.

29. S. autumnalis: culm compressed, ancipitous ; umbel compound; involucrum 2-leaved; spikes lanceolate, acute, somewhat 4-sided.

HAB. Boggy grounds. July-Oct. 2f. Culm 8-12 in. high; leaves long; umbel loose; glumes ovate, mucronate, carinate.

$\uparrow+$ Style bulbous and compressed at the base, ciliate on the margin.

33. S. ferrugineus: culm compressed, scabrous above ; involucrum unequally ciliate; spikes lanceolate, acute, nearly terete; glumes pubescent.

$\mathrm{HAB}$. In wet or dry situations. Aug. Culm $2 \mathrm{in}$. -2 ft. high; leaves channelled, long; umbel mostly simple, with elongated rays; spikes brown. 
21. S. spadiceus: culm compressed, nearly naked; umbel terminal; involucrum rigid, 2-leaved, one of the leaves longer than the umbel; leaves subterete; spikes ovate-oblong, terete.

HAB. Salt marshes. Aug. 4. Cespitose, tenacious ; 2 ft. high; culın compressed above; spikes subsylind, brown.

\section{SCHOENUS. Bog-rush. Eyperoidece.}

\$. mariscoides: culm terete, or a little sulcate, leafy ; leaves channelled, semiterete ; umbel terminal ; fascicles of spikes 3 on each peduncle : seed naked, rounded at the base.

IIAB. Bogs. July. 4. Culm 2 ft. high; fascic. about 12-spiked; spikelets lanceolute; glum. ovate.

\section{RHYNCHOSPORA. Cyperoidea.}

1. R. alba: spikes in corymbose fascicles; culm triangular above; leaves setaceous; seed somewhat lenticular; bristles about 10 .

11ムB. Bogs and swamps July-Sept. 4. Fasc. axill. and terminal, bracteatc; gium. white; seed substipitate.

2. R. fusca: culm triquetrous ; leaves linear, carinate , fuscicles of spikes alternate, pedunculate; spikes. ovate; glumes ovate, brown; seed ovate; with an. acute black tubercle.

UAB. Bogs. 4. Culnn 2 ft. high; glum. mucronate, seed brown, rugose.

3. R. glomerata: spikes in corymbose fascicles, very distant, by pairs ; culm obtusely triangular; leaves flat ; seed obovate-cuneiform, very smooth, as long as the tubercle.

IIAB. Swamps and bogs. July-Sept. Culm leafy; leav. carinate; spik. lat. and term.; glum. brozen..

1. R. capillacea: spikes $3-5$, nearly terminal; culm. triquetrous, somewhat leafy; leaves setaceous; seed stipitate, a little rugose.

HAB.. Sandy swamps. July. 4. A span high , glum. brown; bristles 6 , longer than the seed.

5. R. sparsa: culm triquetrous, leafy; corymbs axillary and terminal, compound, loose ; peduncles capillary, 
seed obovate, rugose, much longer than the iubercle.

HAB. Bogs. July 24. Panicles spreading; the upper one larger; glam. brown; seed white.

i. R. cymosa : culm triquetrous, leafy ; cymes terminal and axillary; the terminal one much larger; seed round-ovate, compressed, transversely rugose ; tubercle conical.

HAB. Swamps. July. 24. A foot or 18 in. high; involucr. long, 2-3-leaved; tubercle white; bristles short.

\%. R. laxa: culm triquetrous; corymbs leafy, decom. pound,loose ; spikes subulate; seed obovate, pointed with the long:persistent style.

HAB. River swamps. July. 4. Culm 3-6 ft. liigh; corymbs very large.

\section{MARISCUS. Cyperoidec..}

1. MI. retrofractus: umbel simple; rays long; spikes obovate, retrorsely imbricate; spikelets subulate, at length bent backward; involucrum 3-leaved.

HAB. Wet meadows. Aug.-Sept. 2f. Culm nearly naked, pubescent; umb. 6-8 rayed.

¿. M. ovularis : umbel simple; rays short; spikes globose ; flowers spreading.

HAB. Boggy grounds. July-Aug. 2f. Root bulbous; culm 6-18 in. high; spikes $3-5-1$; middle one sessile.

ß. tenellus : culm acutely triangular, slender; invo. lucrum 3-5-leaved; 3 of the leaves many times longer than the umbel.

IIAB. Sea-coast. Aug. \& foot high; heads of 10 12 spikelets.

\section{8: DULICHIUM. Cyperoidea.}

D. spathaceum: culm terete; leaves spreading in 3 directions; spikelets spreading.

HAB. Wet places. Aug.-Sept. 4. Culm very leafy, terete below; ochrea cylind.; racemes axillary.

39. CYPERUS. Cyprus-grass. Cyperoidea.

1. C. inflexus: umbel 2-3-rayed, or conglomerate and simple; involucrum 3-leaved, very long; spikelets . 
collected into ovate heads, oblong, 8-flowered; glumes squarrose at the tip.

HAB. Banks of rivers. Aug.-Sept. of ? $2-3 \mathrm{in}$. high; odorous; heads many-fl.; glum. striate, yellow. ish.

9. C. flavescens: spikes linear-lanceolate, in fascicles of 3-4; glumes obtuse; style 2-cleft; seed lenticular; involucrum 3-leaved, longer than the spikes.

HAB. Boggy grounds. Aug.-Sept. 4. A span high ; spikelets 14-20-fl., yellowish.

3. C. Nuttallii: culm acutely triangular; umbel radiate, or nearly sessile, loose; rays short; involucrum 4-leaved; 2 of the leaves very long; spikelet. linear-lanceolate, compressed, acute ; stamens 2 ; style 2-cleft ; seed oblong, obtuse, compressed.

HAB. Borders of salt-marshes. Aug.-Sept. 4 . Cespitose, 5-12 in. high; spik. very acute; glum. green and brown; seed smooth.

1. C. diandrus : culm slender, obtusely triangular;; umbel sessile, or 1-2-rayed; involucrum 3-leaved ; 2 of the leaves much longer than the umbel; spikelets lanceolate-oblong, much compressed, many-flowered $(14-16 ;$ ) glumes margined, rathes acute, keeled; stamens 2 ; style 2 -cleft; seed oval compressed.

IIAB. Salt marshes. Sept. 4.. Culm reak, not cespitose; glum. much compressed, with a broven margin.

5. C. dentatus : umbel compound, (6-10 rayed;) invo. lucrum 3-leaved, longer than the umbel; spikelets 3 on each ray, alternate, ovate, compressed, 8. flowered; glumes acute, nervose, spreading at the points ; seed triquetrous.

HAB. Banks of rivers and sandy swamps. Sept. u. Root tub.; spik. appearing dentate, brozenish; soine. times viviparous.

i. C. erythrorhizos: umbel decompound; involucrum 3-6-leaved, very long; spikes alternate, linear, horizontal, about 12-flowered, nearly terete; style 3-cleft; seed triquetrous.

II $\mathrm{AB}$. Wet places. Aug.-Sept. 4 . Root fib. red; partial umb.naked; spik. at length terete.

7. C. strigosus: spikelets linear, compressed, acute; 
alternate; glumes lanceolate, nervose ; umbel simple, or compound; involucrum about 6-leaved. pubescent on the margin; root globose.

HAB. Wet grounds. Aug.-Sept. 4 . 1-2 ft. high; umb. ochreate; spik. 10-12 fl. yellowish.

8. C. tenuis: spikes lanceolate, acute, 10-17-flowered; umbel simple; involucrum 3-4-leaved, longer than the rays; root tuberous.

HAB. Culm a span high, slender; spik. yellow; glum. nervose.

3. C. phymatodes: umbel simple or decompound; involucrum 3-9-leaved; 3 of the leaves very long; peduncles compressed; spikelets distıchous, linear, the lower one branched, about 15-flowered; sides rather convex; glumes oblong, obtuse; radicles tuberous at the extremity.

IIAB. Wet sandy places. Aug. 4 . Root creeping : leav. mostly rad. spreading, carinate; umb. ochreate.

10. C. compressus: spikes digitate, somewhat by fours ; lanceolate; glumes mucronate, broad, membranaceous.

HAB. Low meadows. 4. Culm 3-8 in. high, obtus. triang.; spik. subsessile, 16-27 $f$.

11. C. virens: culm acutely triangular; umbels decompound; spikelets ovate-lanceolate, in compact heads; involucrum very long.

HAB. Wet meadows and woods. 4. 1-2 ft. high : spik. 16-fl., laterally compressed; glum. acute.

19. C. inariscoides: umbel simple, or with $1-2$ rays: spikelets collected into globose heads, lincar ${ }_{9.7}^{17}$ 8-flowered; glumes loose, obtuse.

IIAB. In dry situations: July-Sept. 4. Root tub.; culm 8-12 in.long; head sometimes solitary , spik. at first terete.

13. C. odoratns: spikelets corymbed, subulate, remote, distichous; glumes somewhat distant; smaller umbels spreading widely, about the length of their: involucels.

HAB. Banks of rivers. +

14. C. flavicomis: culm triquetrous ; umbel compound spikes linear-lanceolate, 7-flowered; glumes ob. tuse ; involucrum very long, reflexed. 
HAB. Boggy woods. 4. Involucr. 5-leav.; spikelets distichous, compressed, yellowish; seed black.

40. ERIOPHORUM. Cotton-grass. Cyperoidece. * Spike solitary.

1. E. alpinum: culm triquetrous, somewhat scabrous, naked; leaves very short, subulate, ; spike oblong; woolly hairs crisped, few.

HAB. High mountains and bogs. July. 4. Culm 8-10 in. high, sheathed; glum. oblong-lanc.: hairs white, very long.

2. E. vaginatum: culm terete below, obtusely triangular above, smooth and rigid; sheaths inflated; spike oblong-ovate; glumes scarious; wool straight, dense.

HAB. Sphagnous swamps. July. 4. Cespitose, a foot high; leaves numerous; glum.livid; wool white.

* * Spikes numerous.

3. E. polystachyon: leaves flat, triquetrous at the extremity; culm nearly terete; spikes on scabrous peduncles, nodding.

HAB. Swamps. July. 4. Culm 1-2fi. high, leafy; leav. very long; spik. 9-12, subterm.; wool rehite or reddish.

t. E. virginieum: culm nearly terete helow, obtusely triangular above; leaves flat, very long; spikes clustered, erect, nearly sessile; involucrum 2-3leaved.

HAB. Swamps. July. Culm 2-4 ft. high; spik. ovate, somerohat umbelled; wool reddish.

B. gracile: culm very slender; leaves almost filiform. HAB. Cedar swamps. Aug.

5. E. angustifolium: culm somerwhat triangular; leaves channelled and triquetrous; peduncles very smooth.

HAB. Wet meadows. 2f. A foot or more high, leav. very narrow; spik. $3-5$; wool white.

\section{FUIRENA. Cyperoidece.}

F. squarrosa: spikes clustered, $(5-6)$ ovate ; culm smooth; leaves ciliate; sheaths hairy; valves of the corolla cordate-ovate. 
HAB. Bog-meadows. Aug. 4. A foot and a halj high, angul.; leav. pubesc.; spik. lateral anu term.; brist. 3-4.

3. pumila : culm pubescent above; leaves smooth; throat of the sheaths hairy; spikes $1-3$, terminal. HAB. Wet sandy places. Aug. Culm 3-6 in. high: spikes mostly 3 ; pet. ovate-lanceol. ; brist. 3-4.

42. CENCHRUS. Burr-grass. Graminea.

C. echinatus: spike with the flowers approximated; involucrum subglobose, villose; culm compressed. HAB. Sandy arid soils. Aug. ○. Erect, or procumb., 1-2 ft. long; involucrum spiny, cartilaginous.

3. tribuloides: spikes and margin of the sheaths white, pubescent.

HAB. Sea-coast and near salt-water. Aug. $\odot . A$ foot high; leav. conduplicate; rachis and involucr. very white.

\section{SPARTINA. Marsh-grass. Graminece.}

1. S. cynosuroides: spikes numerous, $(10-40$, pedunculate, panicled, spreading; leaves broad, flat, at length convolute; calyx with a short awn on one of the glumes; style 2-cleft at the summit.

HAB. Salt marshes. Aug. 4 . 4-9 ft. high; leaves 2-4 ft. long; $f$. closely imbricate; style bifid.

2. S. juncea : leaves distichous, convolute, spreading ; spikes few, 1-3, pedunculate; peduncles smooth; corolla rather obtuse; styles 2 .

HAB. Sea coast, and salt marshes. July-Aug. 4. Root creeping; culm 18 in. high, slender, rigid; cal. very unequal; anth. purple.

3. S. glabra: leaves concave, erect; spikes alternate, sessile, erect, appressed; corolla nearly smooth on the keel; style cleft about half way down.

HAB. Borders of salt-marshes. Aug.-Sept. 4. Very smooth, 3-5 ft. high; spikes 8-14; anth. yellow.

44. ORYZOPSIS. Graminece.

o. asperifolia: culm nearly naked; leaves erect. 
rigid, pungent at the point ; flowers in a racemose panicle.

HAB. Mountain meadows. Apr.-May. i. Culm 18 in. high; rad. leaves long; culm leaves few, very short; pan. very simple; cor. white, hairy; seca large, rhite.

\section{DIGYNIA.}

45. LEERSIA. Rice-grass.

1. L. virginica: panicle simple; the lower branches diffuse; flowers appressed, monandrous, sparingly ciliate on the keel.

HAB. Wet woods and swamps. Aug. 4 . Culm 2-4 ft. high, genicul.; sheaths scabr.; flowers clasping the pedicels, punctate.

2. L. oryzoides: panicle diffuse, sheathed at the base; flowers triandrous, spreading; keel of the glumes conspicuously ciliate.

HAB. Ditches and swamps. Aug.-Sept. 4. Culm $3-5 \mathrm{ft}$. high; leav. and sheaths very scabr.; $f$. whitish.

\section{PASPALUM.}

1. P. ciliatifolium : culm decumbent; leaves hairy and ciliate; sheath hairy; spikes 1-2, rather loose, indistinctly 3-rowed.

IIAB. Sandy fields. Sept. 4. A foot and a half long, simple; term. spik. on a long pedunc.; fl. plano-convex, on bifid pedicels; rach. flex.

2. P. setaceum: culm erect; leaves and sheaths villose; spike generally solitary; flowers in 2 rows.

HAB. Sandy fields. Sept. 4 . Culm 1-2 ft. high. simple; rachis convex on the back.

3. I'. lave: erect, very smooth; leaves short; spikes numerous, $(3-6$,$) alternate ; flowers 2-rowed,$ smooth.

HAB. Banks of rivers. Sept.-Oct. 4. $2 \mathrm{ft}$. high ; leaves broad-linear; spik. a little spreading; pedicels 1-fl. subovate, large.

1. P. stoloniferum: spikes in elongated racemes, some 
what verticillate,spreading; flowers serrulate-ciliate, transversely rugose; leaves short, subcordate; culm procumbent at base.

HAB. Cedar swamps. July-Aug. 4. Sioloniferous; spik. 3-50-fl.; fl. alternate.

47. MILIUM. Millet-grass.

*. M. effusum: panicle diffuse, compound; branches horizontal; glumes ovate, very obtuse; corolla awnless, smooth and shining; leaves broad-linear.

HAB. Woods. July. 4. 5-8ft. high, smooth; panic. alternate, loose.

9. M. amphicarpon: leaves linear-lanceolate, hairy, ciliate; panicle simple, contracted, bearing perfect flowers ; fertile flowers in solitary, elongated, radical scapes at length subterraneous.

HAB. Sandy swamps. Aug.-Sept. 4. Cespitose, 1 -2 ft. high, assurgent; panic. appress., rad. $f$. at length ventricose; glum. acuminate.

3. M. pungens: culm erect; leaves lanceolate, very short, pungent, at length involute; panicle contracted; branches generally by pairs, 2-flowered; flowers awnless, ovate; corolla hairy.

HAB. Rocky hills. May. 4 . 12-18 in. high, rigid; culm leaves very short; panic. fero-fl.; style 2-parted; nect. lanceolate.

\section{PIPTATHERUM.}

P. nigrum : panicle simple; flowers racemose, ovatelanceolate ; corolla black, hairy ; awn as long again as the valves.

HAB. Mountains and rocky places. Aug. 4. Culm 2-3 ft. high, leafy; panic. ferv-fl. flexuous; nect. ovate-lanceolate.

49. STIPA. Feather-grass.

\. avenacea: leaves setaceous; panicle spreading: somewhat secund; branches mostly by pairs, a little divided; calyx as long as the seed; awn naked.

HAB. Sandy woods. June. 4. 2-ft. high; leaves mostly rad. narrow"; cor. stipit. brozenish; aren 23 in. long. 


\section{ARISTIDA.}

1. A. dichotoma : cespitose; culm dichotomous; flower: racemose-spiked; lateral awns very short; the intermediate one contorted.

HAB. Fields and dry hills. Sept. ๑. 8-12 in. high, slender; cor. 1-valved; lat. awns straight.

2. A. stricta : culm and leaves straight, erect; leaves pubescent ; raceme long, somewhat spiked, crowded; awns twice as long as the corolla, spreading.

HAB. Sandy soils. 4. Cespitose, 2-3 ft. high; leav. long, convolute at the extrem.; cor. hairy at base.

3. A. purpuracens: culm filiform, erect, simple; leaves very narrow, flat; flowers in a long spiked panicle; awns nearly equal, twice the length of the corolla, divaricate.

HAR. Sandy fields and woods. Sept.-Oct. 4 . 2 ft. high; leav. filiform at the ext.; panic. many-fl., purple.

\section{MUHLENBERGIA.}

II. diffusa: culm diffuse, (decumbent;) leaves linearlanceolate; panicle branched, appressed; awns as long as the corolla.

IIAB. Dry hills and fields. Aug.-Sept. 4. Culm genic., slender; pan. lat. and term.; cal. scarcely! apparent.

\section{TRICHODIUM.}

1. T. laxiflorum: culm erect; leaves setaceous, and with the sheaths somewhat scabrous; panicle diffuse, capillary, with trichotomous branches; calyx uneqnual.

IIAB. Dry swamps and fields. May-June. 24. A gaot and a half high, filif.; pan. at length pyramidal. purplish.

$\therefore$ 'T. scabrum: culm geniculate at the base, assurgent, brauched; leaves linear-lanceolate, flat, striate, scabrou: sheaths mostly smooth ; panicle verticillate and divaricate; calyx unequal; corolla ovate, acute. 3-nerved.

HAB. Dry woods. Aug.-Sept. H. 12-18 in. High ; 
leaves broad, pale green; panic. concealed ai thic base.

3. T. elatum : culm erect, firm; leaves narrow-linear, flat, scabrous; sheaths smooth ; panicle verticillate, a little spreading; glumes nearly equal.

HAB. Sandy swamps. Aug. 4 . Cespitose, $3 \mathrm{ft}$.high, firm; panic. purp.; cor. shorter than the calyx.

4. T. montanum: culm cespitose, erect; leaves involute, filiform, and as well as the sheaths, scabrous; panicle capillary, loose, a little spreading; glume equal.

HAB. Nountains. July. 4. 8-12 in. high, filiform; panic. elongated, semiverticill., flex. hispid; glum. nerveless; cor. longer than the calyx.

\section{AGROSTIS. Bent-grass.}

1. A. stricta : panicle elongated, straight; calyx equal : corolla smaller than the calyx; valves unequal, with an awn at the base of the inferior valve, longer thar: the flower.

HAB. Fields. June. Culm erect, with black nodes; aren trice as long as the flower.

2. A. vulgaris: branches of the panicle smoothish, at length divaricate; inferior valve of the corolla 3 nersed; stipule very short and truncate.

HAB. Meadows and pastures. June-Aug. 4. Root creeping; culm 12-18 in. high; pan. cupill.; $f$ !. purp.

3. A. alba: branches of the panicle hispid, spreading, loose; inferior valve of the corolla 5 -nerved; stipule oblong.

HAB. Fields and pastures. June-Aug. 4. Root creeping; culin assurgent, ofien sobolif.; panic. capillary.

4. A. lateriflora: culm erect, branched above, soboliferous at the base; panicles lateral and terminal, contracted, dense; calyz acuminate ; rorolla shorter than the calyx, equal, pubescent at the base, awnle-s.

HAB. Swamps and wet meadows. Aug.-Sept. 4. Root creeping; culm $2 \mathrm{ft}$. high, with swelling nodes: ponicles spitie-form. 
3. filiformis: panicles very slender; corolla nearly equalling the calyz.

HAB. Swamps and wet places. Sept.

j. A. soboliferr: culm erect, branched; panicle contracted, fliform, simple; branches appressed; corolla longer than the eqmil calyx, hury at the base; valves equal, awn!ess, mucronite at the tip.

HAB. Rocky hilis and woods. Aug.-Sept. 4. Culm sobolif., sometimes decu nb., $2 \mathrm{fl}$. high; nodes not swelling.

"j. A. tenuiflora: culm nearly simple, pubescent about the joints; branches (if any) appr.ssed; stipules obsolete; panicle contracted, filiform; corolla longer than the calyx; inferior valve with an awn twice as long is the flower.

HAB. Rocky woods. July-Aur. 4. Ront creeping; culm $3 \mathrm{ft}$. high; shearhs mosily muiescent.

\%. A. sylvatica: culm erect, much branched, difiuse, smooth ; stipules lacerate ; panicle biliform : corolla longer than the calyx; awns three times as long as the flower.

HAB. Rocky situations. Aug. 4 . Root creeping; culm 2--3 fi. high; sheruths open and smonth.

3. A. compressa: whole plant very smooth; culm erect, compressed, simple; pauicle nhlong. subcontr:icted, with capillary branches; calys equal, shorter than the corolla, acute; corolla rather obtuse, smooth at the basc.

HAB. Sandy swamps. Sep̣t. 4. Root creepingr ; culm sobolif.; leav. Lnear, long, compress.; sheaths carinate; pan. purp.

2. A. serotina: culm filiform, much compressed; leaves very narrow, carinite, erect; pamicle attenuate; caly $x$ unequal, half as long as the awnless rurolla.

HAB. Sancly swamps. Sept. 4. 19-18 in high; leav. almost filif.; pun. slender. flex.

10. A juncea: leaves strlight and erect, convolutely setaceous ; panicle nulong-py ramidal, verticillate ; flowers awnless; calyx half the length of the corolla.

HAB. Barren Eandy places. Oct. 4 . Culn 1-2 ft. high, terete; $h$. purp.; cal. unequal; nect. obo. zale. 
11. A. virginica: culms numerous, procumbent at the base, assurgent; leaves subdistichous, involute, rigid; panicles lateral and terminal, spike-form; the lateral ones concealed; calyx equal, about as long as the corolla.

HAB. Sandy soils. Sept.-Oct. $\odot$ ? A foot high; leav.hairy at base; sheaths swelled; stip. 0 ; anth. purp.

12. A. longifolia: panicle contracted, spiked, generally concealed; corolla much longer than the calyx, subequal, smooth and spotless, without awns; leaves very long, filiform and recurved at the apex. HAB. Sandy hills and fields. Sept.-Oct. 24. Culm 2-4 ft. high; simple, terete; leaves $2 \mathrm{ft}$. long; pan. sometimes exserted; fl. compress.

13. A. clandestina: panicle spiked, partly concealed; corolla unequal, much longer than the calyx, hairy and spotted, slightly awned; leaves very long.

HAB. Dry hills. Sept. 4. Culm $2 \mathrm{ft}$. high, terete; pan. often sooty; glum. carinate.

\section{CINNA.}

C. arundinacea: panicle large, capillary, loose ; leaves broad-linear; culm smooth.

HAB. Swamps and wet woods. Aug.-Sept. 4. Culm 2--5 ft. high; leav. pale green; fl. green.

\section{POLYPOGON.}

$P$. racemosus: panicle dense, conglomerate, interrupted; bristles of the calyx scabrous; corolla unarmed, hairy at the base; culm branched.

HAB. Bog-meadows. Aug.-Sept. 2f. Culm 3-4 ft. high, compress.; branch. appress.; leav. flat; pan. 2 in. long.

\section{TRICHOCHLOA.}

T. capillaris: leaves convolute, filiform, smooth; panicle diffuse, capillary, very slender; pedicels longer than the awns; awns $3-4$ times the length of the flower.

HAB. Sandy pine woods. Sept.-Oct. 4. Cespitose, 2 ft. high, slender; pan. long, purple, very slender. 


\section{ARUNDO.}

1. A. canadensis: panicle oblong, loose; glumes scabrous, pubescent, as long as the corolla; corolla awned on the back; hairs at the base equaling the valves; culm and leaves smooth.

HAB. Bog-meadows. Aug. 4. Culn 3-4 ft. high, mostly simple; inf. sheaths putesc.; pan. purple.

2. A. conrctata: panicle somewhat spiked, conglomerate; calyx a little longer than the corolla, carinate; corolla longer than the pappus, awned a little below the tip.

HAB. Bog-meadorvs. July-Aug. 4. Culm 3-5 ft. high; pan. struight and erect, never spreading.

3. A. brevipilis: pinicle diffuse, capillary ; calyx unequal, shorter thin the corollid, bearded at the base ; corolla equal, awniess, hairy; pappras very short.

HAB. Sandy swamps. Sept. 2f. Culm 3-4 ft. high, terete; pan. pyram. loose; cal. hairy at base.

\section{PSAMMA.}

P. arenaria: panicle spiked; calyx acute; corolla three times as long as the pappus; leaves involute. HAB. Sea-coast. Aug. 24. Roors long, creeping; culm 2-3 ft. high, rigid; pan. cylind. zehite; $f$. compress.

\section{CRYPSIS.}

C. virginica: spike oblong-cylindrical, thick and loberl; culm procumbent and geniculate ; leaves at length involute, rigid, pungent.

HAB. Sandy ficlds. Aug.-Oct. Culm branched, spreading; leav. flat, divaric.; spik. lat. and term.

\section{ALOPECURUS. Fox-tail grass.}

1. A. pratensis : culm erect, smooth; spilse cylindrical, obtuse, lobed; calyx ciliate, somewhat villose, connate below the middle; corolla as long as the callyx.

HAB. Ficlds. June-July. 24. Culm 2-4 ft. high; leav. flut; spik. $1 \frac{1}{2}$ in. long; wwn troice as long as the flower; styl. connate.

2. A. geniculatus: culn ascending, geniculate; spike E ? 


\section{TRIANDRIA.-DIGYNIA.}

cylindrical; glumes a little connate at the base, hairy on the back and margin; corolla truncate; styles free.

HAB. Wet meadows. June. 4. Root creeping; culm 18 in. high, rooting below; awn as long again as the corolla.

3. aristulatus: awn scarcely exserted.

HAB. Wet meadows. June.

\section{PHLEUM. Cat's-tail grass.}

P. pratense: spike cylindric, calyx mucronate-awned; keel ciliate; awn shorter than the calyx; culm erect.

HAB. Fields and pastures. June-Aug. 4. Culm 2-3 ft. high; leav. glauc., smooth; anth. purp.

62. CYNODON. Bermuda-grass.

C. dactylon : culm creeping; spikes digitate, $(4-5$, spreading; keel of the calyx scabrous ; leaves hairy on the margin and towards the base ; sheaths hairy. HAB. Sandy soils. Aug. 4 . Root long, creeping; culm stolonif.; leav. subdistich.; spik. slender.

\section{GYMINOPOGON.}

G. racemosum.

HAB. Sandy fields. Aug. 4. Culm 1-2 ft.high assurgent, leafy ; leav. ovate-lan., spreading, distich. : pan. large, pyram. ; fl. appress., sess.

\section{PHALARIS. Canary-grass.}

1. P. americana: panicle oblong, spiked; glumes boatshaped, serrulate; corolla unequal; rudiments hairy. HAB. Bog-meadows. July. 4. Culm 2-5 ft. high, terete; leaves broad-linear; pan. a little spreading rchen old.

3. P. canariensis: panicle subspiked, ovate; calyx boatshaped, entire at the apex; rudiments smooth. HAB. Pastures. July. ๑. $18 \mathrm{in.} \mathrm{high;} \mathrm{sheaths} \mathrm{in-}$ flated.

65. ANTHOXANTHUM. Sweet-scented rernal-grass. A. odoratum: spike ovate-oblong; flowers pubescent, subpeduncled, shorter than their awns. 
HAB. Meadows, dry woods, \&c. May-Aug. 4. A foot high; leav. pubesc.; pan. spiked; seed black.

66. BRACHYELYTRUM.

B. aristatum.

HAB. Rocky hills. June-July, 24. Root creeping : culin erect, simp., 2-3 ft.high; pan. racemose; arens twice as long as the $f$.

67. PHRAGMITES. Reed-grass.

P. communis: calyx about 5-flowered; florets longex than the calyx.

HAB. Borders of ponds, and swamps. Aug. 2. Culm 6-12 ft. high, thick; leav. 1-2 in. broad; pan. large nodding; $A$. silky.

\section{GLYCERIA.}

1. G. fluitans: panicles secund, slightly branched : spikelets linear, terete, appressed, 3-12-flowered: florets very obtuse, 7-nerved; leaves long, flat.

HAB. In water. June-July. 4. Root creeping : culm 3-5 ft. high, compress., assurg.; pan. long. racemose.

2. G. acutiflora: panicle simple, elongated, appressed : spikelets linear, terete, 4-12-flowered; florets attenuate, acute, indistinctly nerved; leaves short, erect.

HAB. Wet meadows. June. Culm 2-4 ft. high, subcompress.; stip. elongat.; pan. long, concealed at the base.

69. UNIOLA. Spike-grass.

1. U. latifolia: leaves broad and flat; panicle loose, nodding; spikelets all on long peduncles; florets somewhat falcate, monandrous; the lowest one abortive.

HAB. Mountains and rocky woods. Aug. 24. Culm $4 f t$. high; pan. very large; spik. an inch long, flat, ovate.

2. U. gracilis : panicle elongated, racemose, appressed; spikelets 3-flowered; Horets spreading, straight, monindrous; the lowest one abortive.

IIAB. Sindy swamps. Aug. 4. Culm 3-t ft. high, 
very slender; punic. with solitary branches; spik. broad-cuneate; anth. and stig. dark purple.

3. U. spicuta: panicle spiked, straight ; leaves involute, distichous, spreading; spikelets 5-9-flowered ; florets triandrous.

HAB. Salt-marshes, and on the sea-coast. Aug.Sept. 4. Root long, creeping; culm 12-18 in. high; spik. ovate, yellowish-green; nect. obovate, minute.

70. BRIZA. Quaking-grass.

B. media: panicle erect; spikelets at length cordate, about 7-flowered; calyx smaller than the florets.

HAB. Meadows. May. 4. Culm 18 in. high; stip. obtuse; panic. capill.; spik. tumich, purplish ; cor. nerveless.

\section{POA. Meadow-grass.}

1. P. annua: panicle subsecund, divaricate; spikelets ovate-oblong, 5 -flowered; florets free; culm oblique, compressed; root fibrous.

HAB. Fields, pastures, \&c. April-Aug. ○. Cespitose, 6-8 in. long; panic. rather croweded, at length spreading; cur. pubescent, 5-nerved.

2. P. fasciculata: panicle expanding; branches straight, fasciculate, crowded; spikelets oblong, 3-flowered; florets free; calyx minute, unequal; culm oblique, terete; root fasciculate.

HAB. Salt-marshes. Aug. 4. Culm 1-2 ft. high. firm; panic. at first appressed; spik. subracemose; cor. smooth.

3. P. dentata: panicle loose, somewhat spreading; branches capillary, virgate; spikelets lanceolate, 5 flowered; calyx free; florets unequal ; inferior glume obtuse, 3 -nerved; inferior valve of the corolla 5-nerved, 5-toothed at the aprex when old.

HAB. Shady swamps. June-July. 4. Root creeping; culm $3 \mathrm{ft}$. high; leav. and spik. pale green; pan. at first nodding.

1. P. aquatica $\beta$. americana: panicle erect, semi-verticillate, diffuse; branches flexuous, smooth; spikelets linear, 6-8-flowered; florets ovate, obtuse, free; leares broad-linear, and as well as the sheaths, sinooth. 
HAB. Wet meadows. Aug. 4 . Culm 4-5, thick, leafy; pan. very large, at first nodding; spik. purp.

j. P. maritima : panicle branched, somewhat crowded; spikelets about 5 -flowered, terete; florets rather obtuse, indistinctly 5 -flowered; root creeping.

HAB. Salt-marshes. June. 4. A foot high; leav. involut.; pan. erect, somerchat flexuous; spik. linear, purplish; $f$. woolly at base.

6. P. brevifolia: panicle loose; branches in pairs, horizontal ; calyx 3-4-flowered; corolla pubescent ; leaves very short; stipules, acuminate.

HAB. Mountains and woods. April. 4. Culm $2 \mathrm{ft}$. high, erect, and obliq.; inf. leav. very short; spikelets loose; $f$. distant.

7 P. pungens: culm compressed; leaves very short, cuspidate; panicle somewhat simple, spreading; spikelets ovate, 3-4-flowered; florets webbed, rather obtuse, obscurely 5 -toothed.

HAB. Rocky situations. April. 24. Culm $18 \mathrm{in}$. high, subancipit.; culm leaves about 2, erect ; pan. semiverticil., capill.

3. P. pratensis: panicle diffuse; upper leaves much shorter than the smooth sheaths; florets acute, 5 . nerved, webbed at the base ; stipule short, truncate; root creeping.

HAB. Meadows and pastures. May-July. 4. Pan. pyramid.; spik. ovate; glum. unequal.

3. P. trivialis: panicle equal, diffuse, spikelets oblongovate, about 3-flowered; florets webbed at the base, 5 -nerved; culm sheaths roughish; stipule ob. long; root creeping.

HAB. Moist meadows. June-Aug. 4 . Culm 2-3 ft. high, stolonif.; pan. pyramidal; stipule elongated.

10. P. compressa: panicle contracted, somewhat secund; spikelets oblong, 3-6-flowered; florets webbed; glumes nearly equal; culm oblique, compressed; root creeping.

HAB. Fields and dry hills. June-July. 24. Culm a foothigh, assurgent; leav. glauc.; panicle sub-spiked. 3. sylvestris : panicle loose, spreading; spikelets 2-3flowered; culm slender, nearly erect.

HAB. In dry woods. July. 
11. P. serotina: panicle elongated, diffuse, at length somewhat secund; spikelets ovate-lanceolate, 23-flowered; florets a little webbed at the base, yellow at the tip, obscurely 5-nerved; root creeping.

HAB. In wet meadows. June. 4. Culm 2-4 feet high; pan. appress. when young; cal. often $2 \AA_{\text {. }}$; anth. yellow.

12. P. nemoraits: panicle attenuated, weak; branches flexuous; spikelets ovate, about 3-flowered; florets loose, slightly webbed, acute, obsoletely nerved; stipule almost wanting.

HAB. Rocky woods. July. 24. Pale green; pan. capillary; florets at length distant.

13. P. nervata: panicle equal, diffuse; branches weak, and at length pendulous; spikelets 5-flowered: florets free, conspicuou-ly 7 -nerved, obtuse.

HAB. In wet meadows and in ditches. June. iq . Culm 3-4 ft. high; pan. large, many-fl.; spik. ovate; $f$. decidunus; stam. mostly 2.

14. P. elongata: panicle elongated, racemose; branches solitary, appressed ; spikelets ovate, obtuse, tumid, 3-flowered; florets free; stipule almost wanting.

HAB. Wet meadows. June-July. 4. Culm 3 feet. high, dark green; pan. nodding; fl. 5-nerved.

15. P. obtusa: panicle ovate; contracted; spikelets ovate, tumid, 5-7-flowered; florets free; glumes scarious ; corolla ovate, smooth, obtuse; inferior valve indistinctly 7 -nerved; leaves as long as the culm, and with the sheaths smooth.

HAB. Swamps. Aug. - Sept. 4. Culm. 3-4 ft. high, firm; leav. long, dark green; pan. dense, many-fl.: spik. large.

16. P. canadensis : p: nicle large, effuse ; spikelets semiverticillate, flexuous, at length pendulous ; spikelets ovate, tumid, 5-8-flowered; florets free; inferior valve somewhat acute, 7 -nerved; superior valve very obtuse ; stamens 2.

HAB. Swamps. July-Aug. 4. Culm 3-4 ft. high. ; leav. long; pan. at first pyramidal, at length much spreading and pendulous.

17. P. capillaris : panicle very large, loose, expanding, 
capillary ; spikelets 3 -flowered, ovate, acute ; florets free ; culm branched at the base : leaves hairy. HAB. Sandy fields, and dry hills. Aug. ๑. Culm 12$18 \mathrm{in}$. high, branch. at base; stip. bearded; pan. pyramidal, branched, not hairy at the base.

18. P. hirsuta: culm erect, compressed, simple; sheaths hairy; panicle very large, capillary; branches expanding, at length roflexed, bearded in the axils ; spikelets oblong, about 5 -flowered ; corolla ciliate on the margin.

HAB. Sandy fields. July-Aug. 4. Culm firm, straight; leav. longer than the culm; fl. purplish.

3. spectabilis: spikelets linear, 10-15-Howered.

HAB. Sandy fields on the sea-shore. Taller than the preceding, spik. purp. $\frac{1}{2}$ in. long.

19. P. pectinacea: culm cespitose, oblique ; leaves hairy at the base ; panicle capillary, expanding, pyramidal, hairy in the axils; spikelets linear, 5-9. flowered ; florets free, acute; superior valve of the corolla persistent.

IIAB. Sandy places-wet or dry. July-Aug. ๑. Culm 8-12 in. high, genic. or decumb. at the base; pan. large; $f$. purplish.

20. P. reptans: dioecious; culm branched, creeping; panicle fascicled ; spikelets linear-lanceolate, 1220-flowered; florets acuminate.

HAB. Swamps and river banks. July-Aug. $\odot$. Culm a span long, slender; leav. subulate; pan. 1$2 \mathrm{in.} \mathrm{long;} \mathrm{glum.} \mathrm{very} \mathrm{acute.}$

21. Eragrostis: panicle equal, spreading; the lower branches hairy in the axils; spikelets linearlanceolate, 9-15-flc:vered; florets obtuse; root fibrous.

HAB. Sandy fields, July-Aug. ๑. Culm $18 \mathrm{in}$. high, genic. at base; spik. large; lead-coloar; $A$. uften verrucose on the lecel.

\section{KOELERIA.}

1. K. pennsyleranica: leaves flat, and with the sheaths coftly pubescent : panicle oblong, slender, rather loose; calyx 2-tlowered; glumes nearly equal; the inferior oblong, obtuse and slightly raucronate: -uperior value of the corolla scabrous. 
HAB. woods. May-June 4. Culm erect, $2 \mathrm{ft}$. high, simple; $f$. shining.

¿. K. iruncata: leaves flat, smooth; panicle oblong, racemose ; calyx 2 -flowered, with a third abortive floret, unequal ; inferior glume a little scabrous, obtuse ; corolla glabrous.

HAB. Dry woods. June 24. Culm $2 \mathrm{ft}$. high, slender; cal. very unequal; inf. glum. obliquely truncate.

3. major : panicle large, a little spreading; leaves hroad-linear, very long.

\section{URALEPIS.}

U. aristulata: lateral panicles concealed in the sheaths of the leaves; terminal ones partly exsert ; calyx 3 -flowered; bristle of the corolla as long as the lateral cusps.

HAB. Sandy sea-shore, and in fields. Aug. ๑. Cespitose, procumb. at base; leaves subulate, pungent, hairy; fl. axill. and term., purple.

\section{TRICUSPIS.}

T. seslerioides: panicle expanding and flexuous; spikelets ali pedunculate, about 6-flowered, lanceolate; inferior valve of the corolla ovate, 5 -toother. $\mathrm{HAB}$. Sandy fields. Aug. 4. Culm 4-5 ft. high, erect. smooth; pan.very large, purp.; glum. unequal, ovate, mucronate.

\section{FESTUCA. Fescue-grass.}

1. F. Myurus : panicle slender, crowded, equal ; spikelets about 4-flowered; florets subulate, awned, hairy, monandrous.

HAB. Sandy soils. June ๑. Culm 6-8 in. high, genic. at base; leav. subulate; aren troice as long as the $f$.

2. F. tenella: panicle spiked, very simple, secund; spikelets mostly 9 -flowered; bristles shorter than the subulate florets; culm filifiorm, angular above; leaves setaceous.

HAB. Sandy soils and on hills. June. $\odot$. Culms numerous, 8-11 in. ligh, straight; sheaths pubescent; cal. decid. 
3. F. duriuscula : panicle secund, oblong, contracted ; spikelets $5-6$-flowered, nearly terete ; florets terminated by short bristles; root fibrous.

HAB. Fields and pastures. June. 24. Culni 12-18 in. high; leav. narrow; stam. 3.

1. F. rubra: panicle secund, erect, spreading; spilkelets somewhat terete; florets longer than the bristle at their tips; leaves. pubescent above; root creeping.

HAB. Dry soils. June. 24. Culn a foot and a half high, erect, pubesc.; leav. setaceous, very long; glum. red after flowering.

$\therefore$ F. elatior : panicle spreading, much branched, loose ; spikelets ovate-lanceolate, 4-5-flowered; florets slightly armed; leaves flat; root creeping.

HAR. Wet meadows. June. 4. Culm 3-4 ft. high, terete, smooth; stip. very short; pan. large, nodding when old.

ن. F. pratensis: panicle spreading, branched; spikelets linear, many-flowered, acute ; leaves linear; root fibrous.

HAB. Meadows and fields. June-July. 2. Culm 1-2 ft. high, erect, smooth; pan. subsecund, nearly simple; spic. 7-8-fl.; fl. not mucronate.

7. F. nutans: panicle diffuse, at length nodding; branches long, geminate, naked below ; spikelets ovate, 3-5-flowered; florets somewhat obtuse, unarmed, nerveless.

IIAB. Woods and shaded rocky places. June. $\psi$. Culm $3 \mathrm{ft}$. high, dark green; stip. very short; pan. few-fl., scab.; cor. coriaceous.

3. F. fascicularis : culm procumbent, geniculate; panicle subsecund ; branches straight, spike-like; spikelets appressed, 8-10-flowered; florets armed; leaves very long.

IIAB. Mearlows and sandly soils, near the sea. Aug. $\odot$. Cubin much branch. froin the buse; leaves atten.; pan. erect, sprcading.

\%. I. clandestina: panicle partly concealed in the sheaths; branchcs solitary, simple; spiliclets 10 Howered; florets awned.

IIAB. New-York. Ciulm a span high; leav. lin.: spik. sess. und pedunc, cor. term. by a short bristle. + 


\section{CERATOCHLOA.}

C. unioloides: panicle nodding, spreading; spikelets oblong-lanceolate, compressed, 6-8-flowered; florets acuminate, unarmed; sheaths bearded at the throat; the lower ones hairy ; root fibrous.

IIAB. Rich bottom lands. July-Aug. $\odot$ and 4. Culm 12-18 in. high; leav. pubesc.; pan. small; branch. geminate, pubesc.

\section{DIARRHENA.}

\section{D. americana.}

HAB. Banks of rivers. 4. Culm erect, nearly naked, slender; leav. rad.; broad; pan. racemose; spik. about 2-fl. ; $f$. diverging ; cor. smooth; stam. $2-3$.

\section{DACTYLIS. Orchard-grass.}

D. glomerata: panicle secund, glomerate; leaves carinate.

HAB. Ficlds, meadows, Sc. May-June. 4. Culm 2-3ft.high; panic. contract. glum. strongly ciliate.

\section{DANTHONIA.}

D. spicata: panicle simple, appressed; spikelets $7-9$, about 7-flowered; inferior valve of the co. rolla hairy; leaves subulate; lower sheaths hairy at the throat.

IIAB. Dry woods and sandy fields. June-July. $\Psi$. Culm cespitose, $18 \mathrm{in.}-2 \mathrm{ft}$. high ; leav. hairy above; pan. spiked.

\section{TRISETUM.}

1. T. palustre: panicle contracted, nodding, somewhat verticillate ; calyx 2-3-flowered; florets smooth, acuminate; the inferior one swnless; inferior valve of the superior floret bicuspidate, awned below the tip.

HAB. Wet meadows. June-July. 4. Culm 1-2 ft. high, erect; leav. flat, smooth; pan. fero fl., pale green.

9. T. purpurascens: panicle very simple, somewhat racemose, few-flowered; calyx 3-flowered; glumes 
very unequal, entire; culm and leares smooth: stipule very short, truncate.

HAB. Mountain meadows. June. 4. Culm $2 \mathrm{ft}$. high, leafy; pan. sub-racemose; spik. terete; cal. purple.

\section{BROMUS. Brome-grass.}

1. B. secalinus: panicle spreading, a little branched; spikelets oblong-ovate, compressed; florets about 10 , distinct, rather remote, longer than the flexuous bristles, leaves somewhat hairy.

IIAB. Cultivated grounds. June. ๑. Culm 2-3 ft. high; nodes swelled and pubesc. ; leav. dark green ; pan. ovate, flat when old.

2. B. mollis : panicle erect, contracted; spikelets oblong-ovate, somewhat compressed, pubescent ; bristle straight, as long as the corolla; leaves very softly pubescent.

IIAB. Fields and pastures. June. o. Culm $2 \mathrm{ft}$. high, mostly pubesc.; leav. pale green; spik. 5-10-fl.

3. B. purgans: panicle nodding; spikelets lanceolate, terete ; florets hairy ; bristle straight ; leaves smooth ; sheaths hairy.

HAB. Wet meadows and banks of rivers. Aug. 4 . Culm 4 ft. high, sinooth; sheaths retrorsely hairy; spik. 6-8-fi.

1. B. ciliatus: panicle nodding; spikelets oblong, terete, 8-10-flowered; glumes acute, (not mucronate, ) ciliate; corolla hairy; margin villosely ciliate: bristle short.

HAB. Banks of rivers. June. 4. Culm $3 \mathrm{ft}$. high, pubesc. at the joints; leav. pale green, hairy both sides; pan. with 2 liracts at base.

5. B. pubesccus : panicle at length nodding; spikeletlanceolate, terete; florets pubescent; leaves and lower sheaths pubescent.

HAB. Woods. June. H. Culn $4 \mathrm{ft}$. high, hairy below; leaves smooth beneath.

\section{ARRHENATHERUM.}

1. A. avenaceum: panicle equal, notding; awn as long again as the flower; culm geniculate, smooth; root nodose.

IIAB. Fields. June. 4. Root crceping : culm $3 f$. 
high; pan. loose ; fl. brownish, hairy at base; awr contorted.

2. A. pennsylvanicum: panicle attenuated; awn twice as long as the florets; seed villous.

HAB. Fields and open woods. July. 4 . Culm smooth; branches of the pan. short; awn genic.

\section{AVENA. Oat-grass.}

A. precox: panicle in a dense raceme; florets as. long as the calyx; awn exserted; leaves setaceous. $\mathrm{H} \Lambda \mathrm{B}$. Sandy fields. June. $\odot$. Cespitose, 3-4 in. high; sheaths angular; pan. oblong; spik. 2-fl. cal. longer than the $f$.

\section{AIRA. Hair-grass.}

1. A. fexuosa: panicle spreading, trichotomous; branches flexuous; calyx a little shorter than the florets, and about the length of the awn; leaves setaceous; culm nearly naked.

HAB. Rocky hills. June. 4. Culm $1 \frac{1}{2}-2$ ft. high; leav. mostly rad.; pan. capill.; cor. hairy at base.

2. A. cespitosa: panicle diffuse ; fiorets about the length of the calyx; awn short, straight; leaves flat.

IIAB. Swamps. June. 4. Culm cespit., 18 in. high; leav. very narrow; pan. capill.; cal.blueish.

3. A. aristulata: panicle capillary, spreading; branches verticillate and flexuous ; calyx 2 -flowered, shorter than the Horets; superior floret pedicellate; inferior valve of the corolla truncate, laciniate, with an awn a little below the middle scarcely exserted.

1. A. pumila: panicle small, fastigiate, few-flowered; pedicels short; florets awnless, obtuse, twice the length of the calyx; valves with membranaceous. margins; leaves flat, smooth; culm erect, scarcely longer than the leaves.

IAB. In barren clayey soil. June. 4. An inch high, grozing in tufts.

\section{LOLIUM. Darnel.}

1. I. perenne: florets much longer than the calyx, unarmed, linear-oblong, compressed ; root perennial. II AB. Meadows and road-sides. May-June, H. 
Root creeping; culm $18 \mathrm{in}$. high, erect; leaves smooth; spike 6 in. long ; rach. flex.

2. L. temulentum: florets shorter than the calyx, as long as the bristle at their extremity ; root annual; culn scabrous above.

HAB. Meadows. July. Root fib.; culm 2 ft. high: spikelets much compress., 5-7-f.

\section{ELEUSINE.}

F. indica : spikes straight, erect, in pairs or quarternate; rachis linear ; spikelets lanceolate, about 5 flowered; culm compressed, declined.

$H A B$. Cultivated grounds, and road-sides. JulyNovem. ๑. Culn 8-12 in. high; leav. distichous; spil. gen. in pairs; rach. compress.

\section{TRITICUM. Wheat.}

I. ustivum: spike parallel, compressed; glumes 4How ered, ventricose; bristles generally much longer than the florets.

IIAB. In neglected tields. June. $\odot . \quad \mathrm{Culm} \mathrm{3-4} \mathrm{fi.}$ high; rach. articulat.; brist. 2-3 in. long.

\section{AGROPYRON.}

1. A. repens: spikelets oblong, 5-flowered; glumes subulate, many-nerved; florets acuminate ; leaves flat; root creeping.

IIAB. Fields and cultivated grounds. July. H. Root artic., white, very long; culm $2 \mathrm{ft}$. high ; fl. sometimes with a short bristle.

9. A. caninum: spikelets about 5 -flowered, compressed; glumes 3-nerved, and, as well as the florets, armed with a bristle at the tip; root fibrous.

IIAB. Delaware. 4. Culm 2-3ft. high; spik, sometimes 6. l. $_{\text {; }}$ bristle longer than the flowers.

\section{SECALE. Rye.}

S. cercale: glumes and bristles scabrous-ciliate; corolla smooth.

IIAB. Neglected fields. June. $\odot$ or \$ . Culm 3--1 ft. high; glum. ciliate, subulate; bristle long. F 2 


\section{ELYMUS. Lyme-grass.}

1. E. virginicus: spike erect, coarctate; spikelets in pairs, 2-3-flowered; florets smooth; glumes lanceolate, nerved.

HAB. Banks of rivers. July-Aug. 4. Culm 3-4 ft. high; leav. bright-green; spik. stiffy erect.

Q. E. canadensis : spike nodding at the extremity, patulous ; spikelets 3-5 flowered; florets hairy; glumes linear-lanceolate.

HAB. Banks of rivers. Aug. 4. Root creeping culm 3-4 ft. high; leav. dark-green; spik. gen. pendulous at the extrem.

ß. glaucifolius : leaves glaucous.

HAB. Rocky shores. Aug.

3. E. villosus: spike a little nodding at the extremity, patulous; rachis and florets hispid-pilose; spikelets geminate, 2-3-flowered; glumes linear, pilose-ciliate, 2-nerved, shorter than the florets.

HAB. Dry hills. July. 4. Culm 2-3. ft. high; learcs long, 4--5 lin. broad; spike at first erect.

1. F. kystrix: spike erect; spikelets diverging; calys 0 .

HAB. Rocky hills. July. 4. Culm 3 ft. high; leav. often glauc.; rach. flex.; spik. 3 at each joint; cal sometimes 1-2-leaved, or a rudiment.

91. MELICA. Melic-grass.

M. speciosu: smooth; panicle loose, erect, few-flow ered; branches simple; florets obtuse.

HAB. Mountains. June. 4 . Culm 3-4 ft. high lear. flat, pubes. beneath; pan. subsecund.

\section{ATHEROPOGON.}

A. culudoides : spikes racemose, distant, pendulous : perfect floret with the inferior valve tricuspidate: lateral bristles of the abortive floret half the length of the terminal one.

IIAB. Rocky hills. Aug. 4. Culm 18 in. high, assur. gent; leav. at length involute; spik. 20-40, oblong: sess.; anth. red.

93. PANICUM, Panic.grass.

1. P. Crus galli: racemes alternate and in pairs, com 
pound; rachis 5-angled; glumes terminating in hispid bristles; sheaths glabrous.

HAB. Along ditches, and in cultivated grounds. Aug. -Sept. $\odot$. Culm 2-4 ft. high; leav. broad and flat; pan. dense; bristles sometimes roanting.

2. P. hispidum: panicle compound, nodding; racemes alternate; glumes terminating in hispid bristles; sheaths hispid.

HAB. Salt-marshes. Sept.-Oct. ๑. Culm 3-4 ft. high, thick; $f$. always with bristles.

3. P. clandestinum: culm with short axillary branches; leaves broad-lanceolate, cordate at the base; sheath: hispid, enclosing the short panicles ; abortive floret neuter, 2-valved; superior valve obtuse.

HAB. Shady moist places. July-Aug. if . Culm. 2-3 ft. high, erect and rigid; leaves 1 in. broad; pan. term. and lat.

t. P. pedunculatum: culm dichotomous; leares broadlanceolate, slightly hairy above, attenuate ; sheath: hispid and papillose ; panicle long-pedunculate, compound, smooth ; spikelets ovate, smooth ; abortive floret 2-valved; superior valve half the length of the inferior.

HAB. Moist places in woods. July. H. Culm 3-4 ft. high, much branched above; leav. very acute; pan. terininal.

j. P. latifolium: culm mostly simple, bearded at the joints ; leaves oblong-lanceolate, smooth, or with the sheaths, somewhat pubescent ; panicle terminal, a little exsert, simple, pubescent ; spikelets oblongovate ; abortive floret antheriferous, 2-valved; superior valve subherbaceous, nearly as long as the inferior, acute.

IIAB. Woods and shady thickets. June-July. 4 . Culm 1 ft.high; leav. 1 in. broad; pan. about $15-f$. downy.

ij. P. scoparium: whole plant softly villous; leaves lanceolate ; panicle erect, compound, setaceous, much branched; spikelets turgid, ovate, pubescent.

HAB. New-Jersey. 4. Culm 2 fi. high, mostly simple; leav. somewhat waved; fl. largest of our species.

7. P. nervosum: culm simple; nodes smooth; leares broad-lanceolate, smooth, a little ciliate on the mar 
gin ; panicle much branched, smooth, many-flowered; spikelets oblong; abortive floret antheriferous, with the superior valve subherbaceous, shorter than the inferior.

HAB. Boggy meadows. July. 4. Culm 3-4 ft. high, smooth; leav. subcoriaceous; pan. pedunc. or sessile; fl. large.

8. P. macrocarpon: culm erect, simple; leaves linearlanceolate, erect, a little hairy beneath; joints naked; sheaths hispid; panicle rather compound, smooth; spikelcts ovate-globose; abortive floret neuter.

IIAB. Banks of rivers. July. 4. Culm $3 \mathrm{ft}$. high; pan. few-fl., flexuous; fl. strongly nerved, subglobose; inf. glume very broad.

7. P. pubescens : erect, much branched, leafy, softly pubescent; leaves lanceolate, ciliate; panicle small, few-flowered, free; spikelets subglobose-obovate, pubescent.

HAB. Woods and fields. June. 4. Culm $18 \mathrm{in.} \mathrm{high;}$ nodes hairy; sheaths retrorsely ciliate; pan. with horizon. branches.

10. P. involutum: culm cespitose, simple, or a little Lranched at the base; leaves erect, somewhat rigid, very narrow, at length involute; panicle simple. few-flowered; florets acuminate; superior valve of the neuter floret very small.

IIAB. Massachusetts. 4. Culm $1 \mathrm{ft}$. high; leav. $\alpha$ little hairy; pan. 10-20-fl.; fl. large.

1. P. depauperatum: culm cespitose, hairy at the joints; leaves linear-lanceolate, smooth or hairy; sheaths pubescent; panicle few-flowered; branches in pairs, one of them 2-lowered, the other 1-flowered.

MAB. Barren sandy soils. May-June. 4. Culm 1 ft. high; lower leav. short; pan. term.; branches tortuous.

19. P. dichotonum: culm much branched and dichotomous above; branches fasciculate; leaves very numerous, lanceolate, smooth, panicle simple, capillary, lax ; abortive floret neuter; superior valve minute, bifid. 
IIAB. Dry woods. July-Sept. 4. Culm erect, 8-18 in. high ; joints hairy; leav. spreading; fl. minute. «. curvatum : culm very tall, rather rigid; branches few and a little curved.

3. fasciculatum : culm low, erect or decumbent ; branches and leaves densely fasciculate; panicles very small, concealed among the leaves.

$\%$ gracile: culm tall, slender; leaves membranaceous.

HAB. $\propto$. In moist meadows. $\beta$. In sandy fields. $\gamma$. In swamps.

13. P. nitidum : culm slender, simple, erect, smooth; sheaths bearded at the throat; leaves very few, broad-linear; panicle capillary, rather crowded, compound, remote, smooth; spikelets minute, obtuse, ovate, slightly pubescent; inferior glume very small. HAB. Meadows and woods. June-July. 4. Culm 18 in. -2 ft. high, mostly simple; nodes annulate; leav. a little shining.

\%. ciliatum: culm hairy; leaves linear-lanceolate, (the lowest ones broader,) sparingly hirsute, ciliate on the margin; panicle with the branches and flowers pubescent.

3. ramulosum: culm more branched; panicle contracted; branches smooth.

\% gracile : culm very slender, smooth; leares rery narrow, and with the sheaths smooth; panicle nearly simple, few-flowered, smooth; superior valve of the abortive floret minute, entire.?

d. pilosum : culm simple, very hairy ; lower leaves approximate, and broad, lanceolate ; upper ones linear, rather rigid, somewhat hairy on the upper surface, ciliate at the base ; sheaths villose and minutely papillose ; panicle sub-contracted; branches virgate, and with the flowers, pubescent.

s. glabrum : smooth on every part, except the basc of the leaves, nearly simple; lower leaves short, approximate, sub-cartilaginous; panicle brancherl, almost verticillate; spikelets large; superior valve. of the abortive floret entire.

ל. barbatum: culm simple, smooth; nodes hairy; leaves linear-lanceolate; sheaths smooth, except on the margin; flowers minutely pubescent. 
HAB. $\propto$. In pine-barrens.. . Sandy swamps. $\gamma$. In

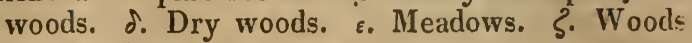
and meadows.

14. P. agrostuides: culm compressed, smooth, erect; leaves very long; panicles lateral and terminal, pyramidal, spreading; branches racemiferous; spikelets appressed; abortive floret neuter, with the valves nearly equal.

HAB. Wet meadows. July-Sept. 4 . Culm 2-3 ft. high; leaves numerous at the root; pan. dark purp.

15. P. virgatum: whole plant very smooth; panicle diffuse, very large ; flowers acuminate; valves of the abortive floret nearly equal.

HAB. Margins of salt marshes. July-Aug. 4 . Culm 3-4 ft. high; leav. long, flat; pan. virgate; spik. large.

16. P. anceps: culm compressed; sheaths ancipitous, pilose; panicle with nearly simple branches; spikelets sub-racemose, much acuminate; abortive floret neuter, with the superior valve bifid.

HAB. In fields. July-Aug. 4. Culm $3 \mathrm{ft}$. high; leav. very long; pan. pyram.; spik. appress.

17. P. rectum: panicle solitary, shorter than the terminal leaf; branches simple, flexuous; spikelets alternate, peduncled, obovate, turgid; glumes striate, acute; leaves linear, straight, tapering to a sharp point, striate and scabrous above, hairy beneath ; sheaths with very long hairs.

HAB. Banks of rivers, July. 4 .

18. P. verrucosum : culm slender, decumbent and geniculate, branched below, and with the leaves smooth; panicle much spreading, few-flowered; flowers verrucose; abortive floret 1 -valved.

HAB. Swamps. Aug. 4. Culm $18 \mathrm{in.} \mathrm{high;} \mathrm{nodes}$ smooth; leuv. bright green; pan. lat. and term.; fl. verrucose.

19. P. proliferum: culm assurgent, geniculate at base, very smooth; panicles terminal and axillary; smooth; spikelets racemose; abortive floret 1 . valved.

HAB. Wet meadows. Sept. 4. Whole plant very 
very smooth; culm 2-4 ft. long, succul.; leav. broad; pan. large; anth. orange.

20. P. capillare: culm nearly simple; sheaths very hairy ; panicle large, capillary, expanding, loose ; spikelets on long peduncles, acuminate, smooth; abortive floret 1 -valved.

HAB. Cultivated grounds. Aug.-Sept. 4. Culm erect, 1-2 ft. high; sheaths hispid; branch. of the pan. reflex. when old.

B. sylvaticum: culm branched at the base, very slender; leaves linear.

HAB. In dry woods. Aug.

21. P. longifolium: whole plant very smooth; culn compressed, erect, simple, slender; leaves very long and narrow ; panicle simple, elongated, racemose ; spikelets acuminate ; abortive floret neuter, 2.valved.

IIAB. Pine barrens. Sept.-Oct. 4. Culm $2 f t$. high ; pan. small, fere-fl.; inf. glume acuminate.

\section{HIEROCHLOA.}

1. H. borealis : panicle subsecund, a little spreading; peduncles smooth; florets unarmed; inferior valve of the corolla ciliate on the margin; root creeping. IIAB. Bog-meadows. May. 4. Srweet-scented; culm $18 \mathrm{in}$. high; leav. smooth and shining; pan. fere-fl.; spik. brown and purple.

2. II. alpina : panicle ovate, contracted; spikelets compressed, longer than the branches; glumes lanceolate, almost nerveless; lateral florets triandrous; one of them with an awn about as long as the valves : sides almost smooth; margin ciliate.

11.AB. High mountains. June. 4. Culm $8 \mathrm{in.} \mathrm{high,}$ smooth, firm, fl. not tumid, larger than in the prereding.

\section{HOLCUS. Soft grass.}

11. lanatus: panicle equal; florets shorter than the calyx, the superior with a recurved awn; root fibrous.

II.1B. Wet meadows. Aug. 4. Whole plant hoarypuliesccnt; culm $18 \mathrm{in}$.high; pan. oblong, contract.; n. whitish. 


\section{SETARIA.}

1. S. viridis: spike cylindrical ; involucrum of $4-10$ fasciculate bristles, scabrous upward; spikelets geminate; perfect floret smooth; sheaths pubescent. HAB. Cultivated grounds. July-Aug. 4. Culm $1 \frac{2}{2}$ ft. high, simp.; leav. smooth; invol. longer than the $f$.

2. S. glauca: spike cylindrical; involucrum of many fascicled bristles, scabrous upward; perfect floret transversely rugose.

HAB. Fields and cultivated grounds. July-Aug. $\odot$. Culm 2 ft. high; invol. yellow zehen old.

3. purpurascens: sheaths hairy; glumes and bristles of the involucrum purple.

3. S. verticillata: spike subverticillate; bristles of the involucrum 2, retrorsely scabrous; spikelets solitary; corolla of the perfect floret nearly smooth.

HAB. Sandy cultivated grounds. July. ๑. Culm 18 in. high, smooth; spike 2 in. long; rach. hispid; invol. purplish.

4. S. italica: spike compound, interrupted at the base, nodding; spikelets conglomerate ; involucrum many times longer than the flowers; rachis tomentose.

HAB. Borders of ditches. July-Aug. ๑. Culm $4 \mathrm{ft}$. high, sub-compress.; pan. 6-8 in. long; inrol. $1-2 \mathrm{in}$. long.

\section{DIGITARIA. Crab-grass.}

1. D. sanguinalis: spikes numerous, fasciculate, a little spreading; leaves and sheaths somewhat hairy ; spikelets oblong; florets pubescent on the margin. HAB. Cultirated grounds and waste places. Aug. 4. Culm decumb. and assurg. ; spik. 4-6 ; rach. flex. ; spikelets by pairs.

2. D. glabra: spikes digitate, sub-alternate, $(3-4$, spreading; leaves and sheaths smooth; spikelets ovate, crowded; calyx equal to the abortive floret, both hairy.

HAB. Sandy fields. Aug.-Sept. ๑. Culm mostly decumbent; spik. much spreading, 2 in. long.

3. D. serotina: decumbent; leaves and sheaths very 
pubescent ; spikes numerous, setaceous; spikelets all pedicellate; inferior glume very minute.

HAB. Fields. 4. Root creeping; culm 12-18 in. long.

4. D. filiformis : culm erect, filiform; leaves somewhat glabrous ; spikes $2-4$, filiform, erect ; spikelets in threes, all perlicellate; calyx 1 -valved, as long as the abortive floret, pubescent.

HAB. Gravelly soils and sandy woods. Aug. $\odot$. Culm simp., 12-18 in. high; leav. short; spik. mostly 2.

\section{ANDROPOGON. Beard-grass.}

1. A. scoparium: spikes simple, lateral and terminal, pedunculate, in pair's ; rachis hairy ; abortive floret neuter; valves awned.

HAB. Barren soils. Aug. 4. Culm $3 \mathrm{ft}$. high; lozver sheaths horry; spik. slend., flexuous.

2. A. virginicum: culn compressed; superior leaves and sheaths smonth; spikes short, $2-3$ fiom each sheath, partly concealed at the base; rachis subterete; abortive flower a mere pedicel without valves; perfect flower monandrous.

HAB. Dry swamps. Sept. 24. Culm 3 ft. high; inf. leav. hairy; rachis pilose.

3. A. macrourum: spikes fisciculate, in dense, lateral, and terminal fastigiate panicles; flowers monandrous ; awn straight; abortive floret without valves. HAB. Swamps. Sept.-Oct. 24. Culı much branched above; spikes vaginate, partly concealed.

1. 1. furcatum: spilses digitate, generally by fours; abortive flower's staminiferous, awnless, resembling the perfect one, the awn of which is subcontorted. JAB. liocky banks. Aug. -Sept. ï: Culm $4 \mathrm{fl}$. high; rad. leav.very long; cal. brownish.

. A. nutans: panicle oblong, hranched, nodding; spike. lets by pairs; calyx hairy, rufescent; awn contorted.

HAB. Fields and hills. Sept.-Oct. 4. Culın 3-t ft. high; jun.large; abort. fl. a rulliment withont valves.

99. HORDEUM. Birley.

H. jubatum : Jateral flowers abortive, neuter; bristleo 
of the calyx and corolla three times as long as the flowers.

HAB. Marshes. §. Culm $2 \mathrm{ft}$. high, slend. sim . bristles of the fl. capillary.

\section{TRIGYNIA.}

100. HOLOSTEUM.

Caryophyllece.

II. succulentum: leaves elliptical, fleshy. HAB. Coldenham, New-York. +.

01. MOLLUGO. Indian-chickweed. Caryophyllea.

M. verticillata: leaves verticillate, cuneiform, acute ; stem branched, depressed; peduncles 1-flowered.

IIAB. Fields. July-Sept. ๑. Stem prostrate, spreading, dichot.; pedunc. axill.; $f$. small, rohite.

102. LECHEA.

Caryophyllec.

1. L. major: plant hirsute on every part; leaves oblong-lanceolate, mucronate ; panicle leafy, pyramidal; branches floriferous towards their extremities : flowers in fasciculate racemes, secund, on short pedicels.

HAB. Dry woods and hills. Aug. 4. Stem erect, often surculose; $f$. minute, round; stam. 3-9.

2. L. minor: whole plant nearly glabrous ; stem assur. gent; leaves linear-lanceolate, acute ; panicle leafy ; branches elongated; flowers on short pedicels.

HAB. Dry woods. Aug.-Dept. Stem slender, 8 in. high; $f$. twice as large as the preceding.

3. L. racemulosa: plant covered with an appressed pubescence; stem erect, with slender, paniculatc branches above; leaves linear, acute, ciliate; flowers rather remote, alternate; raceme naked.

HAB. Sandy fields. +.

4. L. thymifolia: whitish-villose on every part; stem erect; leaves linear, acute ; panicle leafy, elongated; branches short; flowers minute, in lateral and terminal fiscicles; pedicels very short.

HAB. Sandy sea-coast. Aug. 4. Stem a foot high, erect, firm, much branched; leav. vill. at base.

103. PROSERPINACA.

Haloragece.

1. P. paiustris: lcaves linear-lanceolate, serrate; the. lower ones pinnatifid. 
HAB. In water. July-Aug. 4. Root creep.; stem partly submerg., red; fl. sm., axillary.

3. P. pectinacea : leaves all pectinately pinnatifid.

HAB. Sandy swamps. Aug. 4. Leav. finely pectinate; angles of the cap. rather obtuse.

\section{TE'IRANDRIA.}

\section{MONOGYNIA.}

A. Flowers superior.

$\dagger$ Monopetalous.

104. CEPHALANTHUS. Common cal. 0 ; proper minute, angular, 4-cleft. Cor. tubular, slender, 4cleft. Style much exserted; stig. globose. Cap. 2-celled, 2-seeded, (mostly 2-partile.) Recept, globose, hairy.

105. DIPSACUS. Flowers in an ovate or roundish capitulum.-Common cal. (involucrum) many-leaved. foliaceous; proper superior, of one leaf. Cor. tubular, 4-cleft. Seed solitary. Recept. conic, paleaceous. Pappus cyathiform.

106. GALIUN. Cal. 4-toothed. Cor. monopetalous: rotate, 4-cleft. Seeds 2, globose.

107. SPERMACOCE. Cal. 4-toothed. Cor. infundibuliform, 4-cleft. Cap. 2-celled, not 2-parted; cells 1 -seeded, bidentate. Seeds with inllected margins.

108. DIODIA. Cal. deeply 2-cleft, persistent. Cor. tubalar-infundibuliform, 4-cleft. Cap. 2-celled; cells one-seeded.

110. HOUSTONIA. Cal. 4-toothed. Cor. infundibuliform, 4-cleft. Cap. half-superior, 2-celled, 2valved, many-seerled, opening transversely.

111. MITCHELLA. Flowers by pairs upon the same germen. Cal. 4-toother. Cor. infundibuliform; tube cylindric; limb 4-parted, spreading, villous on the inner side. Stam. scarcely exserted. Stig. 4-cleft. Berry, by the union of 2 germens, didymous, 4-secded. 
112. LINNÆA. Cal. double; that of the fruit 2-leaved, inferior; of the flower 5 -parted, superior. Cor. turbinate, subcampanulate, 5 -lobed, equal. Stam. somewhat didynamous. Stig. globose. Berry dry, 3-celled, (only one of the cells bearing a perfect seed.)

$\dagger \dagger$ Polypetalous.

113. SANGUISORBA. Cal. 2-leaved. Cor. 4-cleft, rotate. Caps. quadrangular, between the calyx and corolla, 1-2-celled.

114. CORNUS. Flowers sometimes aggregated in a 4 leaved involucrum.-Cal. 4-toothed. Pet. 4, Drupe with a 2 -celled nut.

115. LUDWIGIA. Cal. 4-parted, persistent. Cor. 4petalled or 0 . Caps. quadrangular, 4-celled, in. ferior, many-seeded.

\section{$\dagger \dagger \dagger$ Apetalous.}

116. ISNARDIA. Cal. campanulate, 4-cleft. Cor. 0. Caps. 4-celled, quadrangular, surrounded by the base of the calyx.

B. Flowers inferior.

$\dagger$ Monopetalous.

117. Plantago. Cal. 4-cleft. Cor. 4-cleft; limb reflexed. Stam. mostly exserted, very long. Caps. 2-celled, opening all round transversely.

118. BARTONia. Cal. 4-parted, appressed. Cor. subcampanulate, 4-parted; segments somewhat erect. Stig. thick, glandular, somewhat bifid. Caps. 1-celled, 2-valved, many-seeded, surrounded by the persistent calyx and corolla.

119. EXACUM. Cal. deeply 4-parted. Cor. 4-cleft; tube globose. Caps. bisulcate, 2-celled, manyseeded, opening at the summit; cells 2 -seeded.

120. SWERTIA. Cal. flat, 4-5-parted. Cor. 4parted; segments spreading, with 2 nectariferous ciliate pores at the base of each. Style short; stig. 2. Caps. 1-celled, 2-valved, acuminate.

121. FRASERA. Cal. deeply 4-parted. Cor. 4-parted, spreading; segments oval, with a bearded, orbicular gland in the middle of each. Caps. com. 
pressed, partly margined, 1-celled. Seeds few, imbricate, elliptic, with a membranaceous margin. 122. OBOLARIA. Cal. 2-parted, bracteiform. Cor. campanulate, 4-cleft ; segments entire (or crenulate.) Stam. subdidynamous, proceeding from the clefts of the corolla. Stig. bifid. Caps. ovate, 1-celled, 2-valved, many-seeded.

\section{$\dagger \dagger$ 4-pctalled.}

123. AMMANNIA. Cal. 1.leaved, campanulate, plicate, 8-toothed, inferior. Cor. 4-petalled or 0 , inserted upon the calyx. Caps. 4-celled, manysecded.

124. PTELEA. Cal. 4-parted. P'et. 4, spreading. Stig. 2. Samara compressed, orbicular, 2-celled, \%sceded.

\section{†† Apetalous.}

125. RIVINA. Cal. 4-parted, persistent. Pet. 0. Berrij 1-seeded. Seed lentiform, scabrous. Stam. 81-12.

12ก. ALCHEMILLA. Cal. 3-cleft; segments spreading, alternately smaller. Cor. U. Style lateral, from the base of the germen. Fruit surrounded by the calyx.

127. SYMPIOCARPUS. Spath ventricose, ovate, acuminate. Spadix subglobose, covered with perfect flowers. Cal. deeply 4-parted, persistent; segments cucullate, truncate, becoming thick and spongy. Pet. 0. Style pyramidal, 4-sided; stig. imple, minute. Seeds solitary, immersed in the spongy receptacle.

\section{DIGYNIA.}

198. HAMAMELIS. Cal. 4-cleft, with 3 bracts (involucrum) at the base. Pet. linear, very long. Nut coriaceous, 2-celled, 2-horned, cleft at the top. Sced 1 in each cell.

\section{TETRAGYNIA.}

124. ILLX. Cal. minute, 4-5-toothed. Cor. rotate, 4parted, or 4-petalled. Style 0 ; stig. 4. Berry 4. - rested. 
130. SAGINA. Cal. 4-leaved. Pet. 4. Caps. 4-celled, 4-valved, many-seeded.

131. TILLÆA. Cal. 3-4-parted. Pet. 3-4, equal. Caps. 3-4, 2 or many-seeded. Stam. sometimes 8,4 sterile.

132. POTAMOGETON. Cal. 4-leaved. Cor. 0. Anth. sessile, alternating with the divisions of the calyx. Nuts 4, 1-seeded, sessile.

133. RUPPIA. Cal. and cor. 0. Seeds 4, pedicellate.

\section{TE'TRANDRIA.}

\section{MONOGYNIA.}

104. CEPHALANTHUS. Button-bush. Rubiacea.

C. occidentalis : leaves opposite or ternate.

HAB. Swamps. July-Aug. Ђ. Shrub $4-5 \mathrm{ft}$. high; leav. ovate-oblong; $f$. wh., in glob. heads.

\section{DIPSACUS. Teasel. Dipsacece.}

D. sylvestris: leaves rarely connate; scales of the receptacle straight; involucrum curved upward.

HAB. Old fields. July. of. Stem 3-4 ft. high, ang. and prickiy; $f$. pale blue, in oval heads.

106. GALIUM.

Bed-straw. Rubiacec.

* Fruit smooth.

1. G. verum: leaves about 8 in a whorl, linear, grooved, scabrous; flowers in dense panicles.

HAB. Pastures. June-July. 2\%. Stem erect, slender; leav. reflexed; fl. yellow.

2. G. trifidum: stem procumbent, scabrous downward; leaves linear, obtuse, scabrous on the margin and mid-rib; those of the stem in fives; of the branches in fours; flowers in terminal fascicles; pedicels short ; corolla mostly 3-cleft.

HAB. Wet places. June-Aug. 24. Stem much branch.; leav. broad-linear; $f$. rh., minute.

3. latifolium: leaves oborate-cuneate.

HAB. Wet places.

3. G. tinctorium : stem diffuse, smoothish; leaves linear, somewhat acute, slightly scabrous; those of the stem in sixes, of the branches in fours; peduncles elongated, mostly 3-flowered. 
HAB. Low grounds. July. 4. Stem a foot high: reak; $f$. white, mostly 4-cleft.

t. G. asprellum: stems diffuse, much branched, retrorsely aculeate; leaves in fives and sixes, lanceo. late, acuminate, aculeate on the nerves and margin ; pedicels short.

IIAB. Shady wet places. June-July. 4. Stem 1-2 ft. high, very leafy; $f$. terminal, white.

* Fruit hispid.

5. G. Aparine: leaves 6 or 8 in a whorl, obovate-lanceolate, hispid above; margin and keel prickly ; stem flaccid, retrorsely aculeate; fruit with uncinate bristles.

HAB. Moist thickets. May-June. ๑. Stem 3-4 ft. long, procumb. or inclin.; fl. white; fruit uncinately lispid.

b. G. brachiatum: stem flaccid, elongated, brachiateramose, hispirl; branches short ; leaves in sixes, oblong-lanceolate, acuminate, smooth; margin and keel setaceously ciliate; flowering branches longer than the whorls, divaricate and dichotomous; pedicels 2-flowered; fruit with uncinate bristles.

IIAB. Meadows and woods. June-Aug. + .

7. F. micranthum: stem much branched, divaricate. retrorsely aculeate; leaves short, lanceolate, mucronate, smooth, margin and keel aculeate ; flowering branches divaricate; pedicels 2 -flowered.

IIAE. Mountain swamps. July. 4. Fl. white, very numerous and sinall; fruit mostly 1 -seeded.

$\therefore$ G. triflorum: stem procumbent, smoothish; leare: in fives and sixes, obovate-lanceolate, mucronate. smooth, scarcely ciliate on the margin; flowering branches elongated, 3-flowered at the extremity: llowers pedicellate; fruit small, hispicl.

HAB. Moist thickets. July-Aug. 4. Stem reak, 3-5 ft. long; leav. membran.; flow. branch. axill and tcrm.

4. (i. pilosum : stem nearly simple, elongated, ascending, with remote joints, hispid ; leaves in fours, oval. mucronate, very hairy on every part, nerveless ; Howering branches elongated, nearly simple, 3Howered at the extremity ; fruit hairy. 
HAB. Dry woods. July-Aug. 24. Stem $1 \mathrm{ft}$. high; leav. obtuse, punctate; $f$. purp.; segments acute.

10. G. circcezans : stem erect, smooth ; leaves in fours, oval, obtuse, smooth; margin and nerves ciliate; peduncles divaricate, few-flowered; flowers remote, subsessile; fruit nodding, with hooked bristles.

HAB. Rocky woods. June-July. 4. Stem $1 \mathrm{f}$. high; branch. at base; leav. 3-nerved; fl. purp.

11. $G$. lanceolatum: stem erect, very smooth; leaves in fours, lanceolate, generally acute, smooth, 3nerved; margin subciliate; peduncles divaricate : fruit sessile, nodding, covered with hooked bristles. HAB. Rocky woods. July. 2f. Stem $1 \mathrm{ft}$. high: leav. 2 in. long, membran. ; $\Omega$. purple.

12. G. bermudianum: stem much branched, scarcely pubescent; leaves in fours, ovate, obtuse: smooth, with pellucid dots; margin and nerves pubescent; flowering branches elongated; fruil uncinately hispid.

HAB. Pennsylvania. June. 24. Leav. linear wehn young, 3-nerved; $f$. panicled; seg. of the cor. lanceol. + .

13. G. boreale: stem straight and erect, branched, very smooth ; branches short; leaves in fours, linearlanceolate, generally obtuse, 3-nerred; margin involute and scabrous; flowers in a terminal panicle. livaricate; fruit minutely and uncinately hispid. HAB. Sandy pine woods. Aug.-Sept. 4. Stem $13 \mathrm{in.} \mathrm{high;} \mathrm{pan.} \mathrm{pyramidal,} \mathrm{crowded;} f$. white.

\section{SPERMACOCE. Rubiacer.}

$\therefore$. diodina: stem diffuse, terete, hairy ; leaves linearlanceolate, nearly smooth; margin and keel serrulate; stipules with numerous long bristles; flower: axillary, sessile, solitary, alternate; fruit hairy.

HAB. Sandy soils. Aug. $\odot$. Stem procunb., 6-8 in. long; leav. oppos.; $f$. white or pale purp.

108. DIODIA.

Rubiaceœ.

D. virginica: smooth; stem procumbent, nearly terete; leaves lanceolate; corolla smooth within: fruit oblong, smooth. 
HAB. Sandy wet places. Sept 4. Stem purp.; leav. oppos.; fl. solitary, oppos., zelite ; cal. subulatc.

109. HEDYO'TIS. Rubiacea.

H. glomerata: stem assurgent; leares lanceolate, pubescent, attenuate at the base; flowers fascicled, axillary and terminal.

HAB. Moist shady places. Aug. ๑. Stem 1-3 in. high, simp. or branch., pubes.; fl. white, minute.

\section{HOUSTONIA. Gentiance.}

H. carulea: stem erect, setaceous, dichotomous ; radical leaves spathulate-ovite; peduncles elongated, 1-flowered; segments of the corolla acute.

HAB. Moist rocks. Apr.-May. 4. Stems numerous, 4-8 in. high; stem leav. narrow; fl. blue.

9. H. longifolia: leaves narrow-lanceolate, tapering at each extremity ; flowers terminill, subsessile ; stem branched, smooth.

HAB. Mountains. June. 4. Stem subfastig. ; $f$. by threes, purp.; segments ovate, acnte.

3. H. purpurea: stem erect, branched above, pubescent at the joints ; leaves sessile, ovate or lanceolate; fascicles of flowers terminal, corymbose.

HAB. Dry woods. July. 4. Leaves broad, rounded at base, 3-nerv.; fl. purp.

1. H. ciliolat $a$ : radical leaves ovate, obtuse, attenuate at the base; margin ciliate; stem-leaves ovatespathulate, sessile; flowers in terminal corymbs, pedicellate; peduncles trichotomous; segments of the calyx linear-lanceolate; stem smooth, branched above.

HAB. Moist rocks. 4. Stem 4-5 in. high; rad. leav. crowedled; $f$. pale purple.

3. H. pubescens : leaves cuneiform, acute, pubescent; the inferior ones semi-petiolate, lanceolate; superior ones semi-oval, sessile; panicle trichotomous. terminal.

HAB. Pennsylvania. +

111. MitChelia. Partridge-berry. Rubiacer.

M. repens. 
HAB. Woods. June-July. 4. Evergreen, creeping, branched, leav. oppos., roundish, petioled. smooth ; fl. term., white, fragrant.

\section{LINNÆA. Caprifolia.}

\section{L. borealis.}

HAB. Mountain woods. July. 4. Evergreen, creeping; leav. oppos., ovate-round; pedunc. erect; $f .2$. drooping, wh. or pale rose-col.

113. SANGUISORBA. Great Burnet. Rosacee.

1. S. canadensis: spikes cylindrical, very long; stamens much longer than the corolla.

HAB. Bog-meadows. Aug.-Oct. 24. Stem $2 \mathrm{ft}$. high, smooth; leav. pinnate; leafl. ovate, serrate; $f$. white.

9. S. media: spikes cylindrical; stamens longer than the corolla; (calyx subciliate.)

HAB. Wet meadows. July-Aug. 4. Leaft. ovatelanceol.; spik. ovate, red. +.

114. CORNUS. Dog-wood, \&c. Caprifolia.

* Flowers capitate, surrounded by an involucrum.

1. C. canadensis: herbaceous; upper leaves verticil. late, veined; leaves of the involucrum ovate, acuminate; drupe globose.

HAB. Mountain meadows and swamps. May-June. 4. Stem 6 in. high; head term., pedunc.; invol. white.

2. C. florida: arborescent; leaves ovate, acuminate; involucrum large, with obcordate leaflets.

HAB. Woods. May-June. Tree 15-30 ft. high ; leav. oppos.; invol. very large, walite or pale rosecol.

$$
\text { * Flowers naked, cymose. }
$$

3. C. sericea: branches expanded; leaves ovate, acuminate, silky-ferruginous beneath; cymes depressed. woolly.

HAB. Shady moist places. June. Shrub $8-12 \mathrm{ft}$. high ; cymes pedunc.; fl. sehite; drupe ovate, blue.

4. C. sanguinea: branches straight; leaves ovate, green on both sides; cymes expanding. 
HAB. Around lakes. June-July. Shrub 8-12 ft. high; leav. broad; drupe dark brown. +.

5. C. circinata: branches verrucose; leaves broad? oval, (orbicular) acuminate, white-downy beneath ; cymes depressed.

HAB. Banks of rivers, woods. June. Shrub $6-8 f t$. high ; cyme crowded, nearly smooth; drupe blue.

6. C. alba: branches recurved, smooth; leaves ovate, acute, pubescent, hoary beneath ; cymes depressed. HAB. Wet woods. - A small tree; brunch. slendler; cymes small; drupe white.

7. C. paniculata : branches crect; leares ovate, acuminate, hoary beneath ; cymes panicled.

HAB. Swamps. June. Shrub 8-12 in. high; branch. punct.; cyme loose; drupe nearly glob., white.

8. C. stricta: branches straight, fastigiate; leaves ovate, green on both sides, somewhat naked; cymes panicled.

HAB. River banks. June. Shrub 8-15 ft. high, stolonif.; bran. red, punct.; drupe blue.

3. C. alternifolia: leaves alternate.

HAB. Shidy woods and swamps. June.-A small tree; branch. mostly verruc.; drupe purp., globose.

115 LUDWIGIA. False Loose-strife. Onagre.

1. L. macrocarpa: stem erect, branched, nearly smooth; leaves attenuate, narrow-lanceolate, pale beneath; peduncles axillary, 1-flowered; capsule globose-quadrangular, winged.

HAB. Swamps. July-Aug. 2f. Stem $2 \mathrm{ft}$. high, purp.; fl. on short pediunc, yellow ; pet. caducous.

3. L. pilosa : stem erect, branched, hairy ; leaves alternate, oblong, sessile; peduncles 1-flowered, axillary ; capsules globose, quadrangular, winged.

HAB. Ditches and pools, in sandy soils. July-Aug.

4. Stem 2 ft. high; leav. 1 in. long; fl. yellow.

3. I. uniflora: stem straight, simple; leaves alternate, lanccolate, acute, glabrous ; flower terminal ; petal.s longer than the calyx.

IIAB. New-Jersey. +. Probably L. nacrocurpa.

116. ISNARDIA. Onagre.

1. palustris : creeping and shining; leaves opposite, 
ovate-lanceolate, petiolate; flowers axillary, sol.tary, sessile; capsule subovate, slightly angled.

HAB. Stagnant waters. June. 4 . Srimming or creeping, succul.; fl. minute, quadrang.

\subsection{PLANTAGO. Plantain. Plantagines.}

1. P. cordata: leaves ovate, cordate, very broad, subdentate, smooth ; spike very long; flowers subimbricated; the inferior ones scattered; bracts ovate, obtuse.

HAB. Wet meadows. June. 4. Leav. 6 in. long, $3-4$ broad; spike 13 in. long; cells of the cap. 2seed.

3. P. major: leaves ovate, smoothish, subdentate, generally shorter than the petioles; scape terete; spike cylindrical, slender; flowers closely imbricate ; capsules many-seeded.

HAB. Fields, waste grounds, \&c. May-Aug. $\boldsymbol{\Psi}$. Leav. spreading; scape 8-12 in. long, pubes.; dissep. of the cap. plane.

3. P. media: leaves orate, pubescent, longer than the petioles; scape terete; spike short, cylindric; cells of the cap-ule 1 -seeded.

IIAB. Fields. July. 4. Leaves spreading; petioles short; spike 1-2 in. long, dense.

^. P. virginica: hiiry-pubescent; leaves lanceolateovate, subdenticulate; spikes cylindrical, with remote flowers : scape angular.

IIAB. Sandy soils. May-June. f. Plant gray, 23 in. high; spike yellowish; cap. 2-sceded; dissep. plane.

5. P. Ianceolata: leaves lanceolate, acute at each end; spike short, ovate-cylindrical; scape angular ; capsule 2 -seeded.

IIAB. Fields, paštures, Sc. May-Sept. 4' Scape 12-13 in. long; spike brownish; stan. very long.

6. P. maritima: leaves linear, grooved, fleshy, hairy near the base; scape terete; spike cylindrical, bracts rather acute.

IIAB. Salt marshes. Aug.-Sept. 4. Scape as long as the leaves; $f$. rather remote; caps. 2-sceded.

¡. $\longmapsto$. pusilla: minutely pubescent ; leaves linear-subulate, flat, entire, acute; scape terete, slender: 
longer than the leaves; spike cylindrical, loose : lower flowers distant ; bracts ovate, acute, as long as the calyx.

HAB. Sandy hills and fields. June. ๑. Scape 2-3. in. long; segments of the cal. subul.; caps. 2-seeded.

8. P. maxima: leaves ovate, subdenticulate, 9-nerved: spike cylindrical, imbricate; scape terete.

HAB. Wet rocky situations. July-Aug. P. cordata?

\section{BARTONIA. Gentiance.}

B. tenella: culm somewhat branched; peduncles opposite, the lower ones branched; segments of the corolla oval, acute, scarcely longer than the calyx ; style very short.

HAB. Swamps. Aug. - Sept. ๑. Stem 3-8 in. high . very slend.; leav. bract-like; fl. small, greenish-wohite.

119. EXACUM. Gentiance.

E. pulchellum: calyx 4-parted; segments subulate: panicle corymbose; peduncles filiform.

HAB. Near the sea-coast. Aug. Flowers rose-colour.

120. SWERTIA. Gentiance. Felwort.

1. S. deflexa: corolla campanulate, with deflexed horn: at the base; leaves ovate; branches short.

HAB. Borders of lakes. Aug. f. Stem $18 \mathrm{in.} \mathrm{high.}$ nearly simp.; leav. oppos., sess.; fl. greenish-yell.

2. S. pusilla : corolla rotate, twice as long as the calyx : stem simple, 1-flowered ; leaves oblong.

HAB. High mountains. June. 4. One in. high; lear:. 1-2 pairs; 7 . large, blue.

121. Frasera. Gentiance. American Colombn.

F. caroliniensis.

IIAB. Borders of lakes. July. 8. Stem 3-5 ft. high: erect; leav. oppos. and verticill.; fl. grcenish-ycll.. punct.

\section{OBOLARIA. Gentiance.}

0. virginiaca.

IIab. Shady forests. Apr.-May. 4. Stem 3-4 in. high. cespit.; leav. oppos., obovate; Al sublerm., whith or reddish, marcescent. 


\section{AMMANNIA. Salicarice.}

1. A. ramosior: stem erect, thick, subterete; leaves lanceolate, dilated at the base ; lower flowers com pactly verticillate.

HAB. Brackish meadows. Aug-Sept. ๑. Stem 4-8 in. higk, simp. or somerehat branched; pet. purp.

9. A. humilis: stem procumbent at the base, branched, slender, quadrangular; leaves lanceolate, alternate at the base; flowers solitary.

HAB. Borders of ponds. Aug. $\odot$ and $\hat{\jmath}$. Stem 4-7 in. high, rarely simp.; fl. small, pale purp.

124. PTELEA. Terebintaceœ.

P. trifoliata: leaves ternate; flowers in panicles: polygamous.

HAB. Shady moist places. June. Shrub $6-8 \mathrm{fl}$. high, spreading; leav. petiol.; fl. greenish-rohite.

125. RIVINA. Atriplices.

R. laevis: racemes simple; leares ovate, acuminate. glabrous, flat; stem tercte.

HAB. Pennsylvania? A shrub with the habit of Phytolacca decundra. Fi. in axill. racemes.

126. ALCHEMILLA. Rosacece. Lady's martle.

A. alpina: leaves digitate, serrate at the extremity. white and sattiny beneath.

HAB. High mountains. 2\%. Fl. white.

127. SYMPLOCARPUS. Aroidece. Skunk-cabbage.

S. foetida.

HAB. Swamps and low grounds. Feb.-Apr. 4. Plant fetid; leaves large, ovate-cord. ; spath. purp., spotted, cucull.; spadix pedunc.

\section{DIGYNIA.}

128. HAMAMFLIS. Berberides. Witch-hazel.

H. virginica: leaves obovate, acute, dentate, cordate, with the sinus small.

HAB. Hoist woods. Oct.-Nov. Shrub 6-12 ft. high; leav. altern., large; fl. axill., clustered, yell. 
13. parvifolia : leaves oblong-ovate, upper part undulately and coarsely crenate; under surface pubescent, somewhat hirsute; segments of the calyx oblong; stamens and perigynous filaments ofter nearly equal.

HAB. Mountains. Smaller than the preceding; $f$. bright yellow.

\section{TETRAGYNIA.}

\section{ILEX. Rhamii. Holly.}

1. 1. opaca: leaves ovate, spinous, acute, smooth, flat ; fascicles of flowers loose, on the base of the younger branches ; peduncles compound.

HAB. Sandy woods. June. An evergreen tree. 10-40 ft. high ; leav. coriaceous, shining; fl. yell.white; berries red.

2. I. canadensis : leares deciduous, ovate, very entire ${ }_{z}$ smooth ; peduncles subsolitary, (or fasciculate, ) very long, 1-flowered; fruit obtusely subquadrangular.

HAB. Rocky hills and mountains. May-June. Shrub 3-5 ft. high; leav. mucron.; fl. dioec., minute; berries red.

\section{TILLEA.}

'T. simplex : stem erect and simple; leaves connate, oblong-linear and somewhat acute; flowers alternate, sessile ; petals erect, twice as long as the calyx.

HAB. Muddy banks of rivers. $\odot$. Plant minute.

131. SAGINA. Caryophyllea. Pearl-wort.

1. S. procumbens: perennial; stem procumbent, smooth; petals very short.

HAB. Near springs. July. Spreading, 2-4 in. long; leav. linear-subul.; pet. sometimes wanting.

2. S. apetala : annual; stem erest, pubescent; leaves cuspidate: flowers alternate; petals very minute or 0 .

HAI3. Sandy fields. May-June. Stems numerons, filif.; lcav. subul.; $f$. on long pedunc. 
132. POTAMOGETON. Naiades. Pond-weed.

* Upper leaves floating.

1. P. natans: upper leaves floating; coriaceous, oblong-ovate, on long petioles, (subcordate;) lower ones membranaceous, lanceolate, tapering to a footstalk.

HAB. Lakes and slow streams. July-Aug. 2f. Upper leav. 2-3 in. long, 2 broad, nerved; spike greenish.

2. P. Aluitans: upper leaves floating, coriaceous, oblong-lanceolate, petiolate, tapering at the base, lower ones linear, sessile.

HAB. Ponds and slow streams. July-Aug. 4. Upper leav. 3 in. long, 1 broad, olive-green; pedunc. thick.

3. P. heterophyllum: upper leaves floating, petiolate, elliptical ; lower ones sessile, crowded, linear.

HAB. Ponds and slow streams. Aug. 11. Half the size of the preceding; submerg. leav. very narroze.

1. P. diversifolium: upper leaves Aloating, elliptical, petiolate, 5-nerved; inferior ones filiform; spike axillary, almost sessile, few-flowered.

HAB. Ponds and slow streams. June. 4. Stems filif.; upper leav. not an in. long; spik. 4-6 fl.

* Leaves all submerged.

5. P. perfoliatum: leaves amplexicaul, cordate, ovate.

HAB. Rivers and lakes. Aug. Stem dichot.; leaves 1 in. long, subpelluc.; spike ferw-fl.

6. P. lucens: leaves ovate-lanceolate, petiolate.

HAB. Rivers and lakes. Aug. 4 . Stem terete; leav. 2-3 in. long, pelluc. reticul. ; spik. cylind.

7. P. crispum: leaves lanceolate, tapering, sessile, undulate and serrate.

HAB. Lakes. Aug. 4. Stem subcomp.; leav. $1 \frac{1}{2}$ in. long; spik. \&-10 fl.

3. P. compressum: leaves linear, obtuse, sessile; stem compressed.

HAB. Slow, streams. July-Aug. 4. Stean much 3ranch., thick; pedunc. compress.; spik.1-6 fl. 
3. P. pauciflorum : leaves sessile, narrow-linear, flat ; spike capitate, 4-flowered; stem slender, terete. HAB. Ponds and rivers. July-Aug. 4. Stem almost filif., much branch.; spik. on short pedunc.

10. P. pectinaceum: leaves setaceous, distichously approximate, sheathing; stipules almost wanting; spike few-flowered, interrupted.

HAB. Ponds. June. 4. Stem flif., dichot.; leav. numerous; 4--6 in. long; pedunc. elongat.

133. RUPPIA. Naiades.

R. maritima.

HAB. Salt marshes, ditches. July. 4. Floating; stem long; leav. setac. flut; spadix naked, 2-fl,; anth. large.

\section{PEN'TANDRIA.}

\section{MONOGYNIA.}

\section{A. Seeds naked. (Asperifolix.)}

* Seeds fixed to the bottom of the calyx.

134. PULMONARIA. Cal. prismatic, pentagonal, 5toothed. Cor. infundibuliform; border 5-lobed; orifice naked. Seeds imperforate at the base.

135. LITHOSPERMUM. Cal. 5-parted; segments acute, carinate. Cor infundibulitorm ; border 5 lobed; orifice naked. Stcm included within the tube of the corolla. Stig. obtuse, bifid. Seeds imperforated at the base, indurated, shining.

136. ONOSMODIUM. Cal. decply 5-parted; segments linear. Cor. subcampanulate; border ventricose, half 5-cleft ; segments connivent, acute; orifice naked. Siyle much exserted. Seeds ovate, shining, imperforate at the base.

1.37. LCHIUM. Cal. 5-parted; segments subulate, erect. Cor. subcampanulate; tube very short; horder unequally 5 -lobed, the lower segment acute and reflexed; orifice pervious. Stig. bifid. Seeds tuberculate, imperforate at the base. 
138. MYOSOTIS. Cal.5-parted, or 5-cleft. Cer. hypocrateriform; segments very obtuse; orifice closed with connivent scales.

139. LYCOPSIS. Cal. 5-cleft. Cor. infundibuliform; orifice closed with ovate, connivent scales. Seeds perforate at the base.

* Seeds fixed to a central column.

140. CYNOGLOSSUM. Cal. 5-parted. Cor. short, infundibuliform; orifice closed with connivent scales. Seeds depressed.

141. ROCHELIA. Cal.5-parted. Cor. hypocrateriform; orifice closed with connivent scales. Seeds echinate, compressed.

B. Flowers 1-petalled, inferior. Seeds in a pericarp. * Fruit a capsule.

๙. Capsule 1-celled.

49. ANAGALLIS. Cal. 5-parted. Cor. rotate, 5lobed. Fil. hirsute at the base. Caps. globose, bursting all round transversely, many-seeded.

143. LYSIMACHIA. Cal. 5-cleft. Cor. rotate, (and subcampanulate,) 5-cleft. Caps. 1-celled, 5-10valved.

14. PRIMULA. Flowers in an involucrate umbel. Cal. tubular, 5-toothed. Cor. hypocrateriform; tube cylindrical ; orifice naked; border 5 -lobed; lobes emarginate. Caps. 1-celled, opening with 10 teeth.

145. DODECATHEON. Cal. 5-cleft. Cor. 5-parted; segments reflexed. Fil. very short; anth. con. nivent. Caps. 1-celled, oblong, opening at the apex.

146. MENYANTHES. Cal. 5-parted. Cor. infundibuliform ; border spreading, 5-lobed, equal, hairy within. Stig. capitate, sulcate. Caps. 1-celled, 2-valved; valves seminiferous on the sides.

14. VILLARSIA. Cal. 5-parted. Cor. rotate, 5-lobed; segments bearded at the base, with their margins inflexed. Glands (nectaries,) 5, alternating with the stamens. Stig. 2-lobed. Caps. 1-celled, 2ralved, many seeded. 
148. HOTTONIA. Cal.5-parted. Cor. hypocrateriform, 5-lobed. Stam. seated upon the tube of the co. rolla. Stig. globose. Caps. 1-celled, globose, acuminate.

149. SAMOLUS. Cal. 5-cleft, half superior. Cor. hypocrateriform, 5 -lobed, with 5 intermediate scales (or sterile stamens.) Caps. half inferior, 1-celled, 5 -tootbed, many-seeded; receptacle unconnected.

150. SABBA'TIA. Cal. 5-12-parted. Cor rotate, 5-12. parted. Stig. 2, spiral. Anth. at length revolute. Caps. 1-celled, 2-valved.

151. HYDROPHYLLUM. Cal.5-parted. Cor.subcam. panulate, 5-cleft, with 5 longitudinal, margined, melliferous grooves on the insiste.

B. Capsule 2-(3-) celled.

152. PHACELIA. Cal. 5-parted. Cor. subcampanulate, 5 -cleft, with 5 longitudinal margined melliferous grooves on the inside. Stam. exserted. Style filiform; stig. 2. Caps. 2-celled, 2-valved, 4-seeded; each of the valves septiferous in the centre.

153. SPIGELIA. Cal. 5-parted. Cor. infundibuliform; border 5-cleft, equal. Anth. convergent. Caps. didymous, 2-celled, 4-valved, many-seeded.

154. VERBASCUM. Cal. 5-parted. Cor. rotate, 5-lobed, unequal. Stam. declined, generally bearded. Stig. simple. Caps. 2-celled, with inflexed valves, many'-seeded.

155. NICOTIANA. Cal. tubular, 5-cleft. Cor. infundibuliform; border plicate, 5-cleft. Stam. inclined. Stig. capitate. Caps. 2-celled, 2-4-valved.

156. HYOSCYAMUS. Cal. tubular; border 5-cleft. Cor. infundibuliform, 5-lobed, irregular; lobes entire. Stam. inclined. Caps. 2-celled, operculate.

257. CONVOLVULUS. Cal. 5-parted, naked, or with 2 bracts at the base. Cor. campanulate, plicate. Stig. 2. Caps. 2-3-celled, with as many valves; cells 1 -2-seeded.

158. IPOMÆA. Cal.5-cleft, naked. Cor. infundibuliform, campanulate, 5-plaited. Stig. canitate ${ }_{2}$ Caps. 2 -3-celled, many-seeded. 


\section{r. Capsule 3-5-celled.}

159. PHLOX. Cal. deeply 5-cleft; segments connivent. Cor. hypocrateriform; border 5-lobed, flat ; tube more or less curved. Stam. very unequal; fil. inserted into the tube of the corolla above the middle. Caps. subrotund, 3-celled; cells 1-seeded. Seeds oblong, concave externally.

160. POLEMONIUM. Cal. campanulate, 5-cleft. Cor. campanulate; border 4-5-lobed, erect; tube short, clused by 5 staminiferous valves. Fil. bearded at the base. Caps. subrotund, 3-celled; cells many-seeded; valves membranaceo-crustaceous. Seeds oblong, somewhat triangular.

161. DIAPENSIA. Cal. deeply 5-parted, with 3 bracts at the base. Cor. hypocrateriform; border 5 cleft. Fil. compressed, inserted into the summit of the tube, and alternating with the segments of the corolla. Caps. 3-celled, 3-valved, manyseeded.

169. DATURA. Cul. tubular, angular, deciduous; base orbicular, persistent. Cor. infundibuliform, plicate. Caps. ovate, 2-celled, 4-valved; cells 2 parted.

163. AZALEA. Cal.5-parted. Cor. short, campanulate, 5-cleft. Stam. equal, shorter than the corolla: anth. opening longitudinally. Style straight, included. Caps. 5-celled, 5-valved, opening at the top.

$$
\text { * Fruit a berry. }
$$

64. PHYSALIS. Cal. 5-toothed. Cor. campanulaterotate. Stam. connivent. Berry 2-celled, covered by the inflated calyx.

165. SOLANUM. Cal. 5-10-parted, persistent. Cor. rotate, 1-petalled, 5-6-lobed. Anth. oblong, opening at the top by 2 pores. Berry $2-6$-celled.

C. Flowers 1-petalled, superior.

* Fruit a capsule.

166. CAMPANULA. Cal. mostly 5 -cleft. Cor. campanulate, 5-cleft. Fil. dilated at the base. Stig. 3-5cleft. Capsule 3-(rarely 5-) celled, open by lateral pores. 
167. LOBELIA. Cal. 5-cleft. Cor. irregular, 5-parted, cleft on the upper side nearly to the base. Anth. united into a tube. Stig. 2-lobed. Caps. inferior, or semi-superior, 2-3-celled, 2-valved at the apex.

168. DIERVILLA. Cal.oblong, 5-cleft, bracteate at the base. Cor. infundibuliform, 5-cleft, spreading. Stig. capitate. Stam. a little exserted. Caps. naked, oblong, 4-celled, many-seeded.

* Fruit a berry.

169. LONICERA. Cal. 5-toothed. Cor. with the tube elongated; border 5-cleft, generally unequal. Stam. exserted. Stig. globose. Berries distinct, 3 celled, many-seeded.

170. XYLOSTEUM. Flowers by pairs on the summit of the same peduncle. Cal. 5-toothed, with 2 connate bracts at the base. Corr. 5-cleft, subequal, or bilabiate. Berries by pairs, more or less connate, 2-celled, many seeded.

171. SYMPHORIA. Cal. minute, 4-toothed, with 2 small bracts at the base. Cor. subcampanulate, short, 5-cleft, nearly equal. Stam. nearly exserted. Stig. globose. Berry crowned with the persistent calyx, 4-celled, 4-seeded; 2 of the cells sometimes abortive.

172. TRIOSTEUM. Cal. 5-cleft; segments linear, nearly as long as the corolla. Cor. tubular, 5. lobed, subequal, gibbous at the base. Stam. in. cluded. Stig. capitate, lobed. Berry 3-celled, 3seeded, crowned with the calyx.

D. Flowers 5-petalled, inferior.

* Fruit a capsule.

173. ITEA. Cal. minute, 5-cleft. Pet. linear, reflexed。 spreading, inserted upon the calyx. Stig. capitate, 2-lobed. Caps. 2-celled, 2-valved, many-seeded. Seeds attached to the inflexed margins of the valves.

174. IMPATIENS. Cal. 2-leaved, deciduous. Cor. irregular. Nect.cucullate, calcarate. Anth. cohering at the apex. Caps. 5-valved, bursting elastically. 
175. VIOLA. Cal. deeply 5-cleft, produced at the base。 Cor. 5-petalled, irregular; upper petal corniculate at the base. Anth. connivent, cohering. Caps. 1-celled, 3-valved.

176. CLAYTONIA. Cal. 2-leared. Pet. 5, emarginate, Stig. 3-cleft. Caps. 1-celled, 3-ralved, 3-5seeded. Seeds reniform.

177 CEANOTHUS. Cal. turbinate, 5-cleft. Pet. 5. saccate and arched, with long claws. Stig. 3. Caps. tricoccous, 3-celled, 3-seeded, 3-parted, opening on the inner side.

178. EUONYMUS. Cal. 5-pacted, or 5-cleft, its base internally bearing a peitate disk. Pet. 5 , spreading, inserted on the outside margin of the glandular disk. Stam. 5. Caps. 5-angled, 5-celled, 5valved, coloured; cells 2 -lobed. Seeds arillate.

179. CELASTRUS. Cal. 5-lober, flat. Cor. 5-petalled. Stam. seated around a 5 -toothed glandulous disk. Caps. or theca obtusely triangular, 3-celled, 3valved; valves septiferous in the centre; cells 1-2-seeded. Seeds covered with a 4 -cleft coloured arillus.

\section{* Fruit a berry.}

180. RHAMNUS. Cal. urceolate, $4-5$-cleft. Pet. $4-5$, opposite the stamens, (sometimes abortive.) Stam. 4-5. Stig. 2-4 cleft. Berry 3-4-seeded.

181. VITIS. Cal. minute, 5 -toothed. Pet. 5, cohering at the apex, deciduous. Stig. sessile, obtuse, capitate. Berry 1-celled, 5 -seeded. Seeds subcordate.

182. CISSUS. Cal. minute, 4-5-toothed. Pet. 4-5, unconnected above, spreading, deciduous. Germ. surrounded with a glandulous disk. Berry 2-4seeded.

E. Flowers 5-petalled, superior.

183. RIBES. Cal. superior, campanulate, 5-cleft. Pet. and stam. inserted upon the calyx. Style 2-cleft. Berry many-seeded.

F. Flowers incompletc.

184. HAMILTONIA. Polygamous. Perfect Fl. Cal. turbinate, campanulate, 5-cleft. Cor. (). Nect. 
with the disk 5 -toothed. Style 1 ; stig. 2-3, sublenticular' germ. immersed in the nectary. Drupe pyriform, 1 -seeded, enclosed in the adbering base of the calyx. STAM. FL. resembling the perfect, except in wanting the pistil.

185. THESIUM. Cal. 1-leaved, tubular-campanulate, 4-5-cleft. Anth. attached to the calyx by a tuft of filaments. Nut 1-seeded, covered with the persistent calyx.

186. ANYCHIA. Cal.5-parted; segments oblong, connivent, callous, and subsaccate at the apex. Cor. 0 . Fil. 3-5, distinct, without intermediate setæ. Stig. subcapitate. Utricutus 1-seeded, covered by the calyx.

187. GLAUX. Cal. campanulate, 5-lobed, coloured. Cor. 0. Caps. globose, acuminate, 1-celled, 5. valved, 5-seeded.

\section{DIGYNIA.}

A. Flowers monopetalous, inferior

* Follicles 2.

188. APOCYNUR. Cal. very small, 5-cleft. Cor. campanulate; border with 5 short spreading or revolute lobes. Anth. saggitate, connivent, cohering to the stigma by the middle. Glandular teeth 5 , acute, alternating with the stamens, and opposite the segments of the corolla.

189. PERIPLOCA. Cal. 5-cleft. Cor. rotate, flat; orifice surrounded with an urceolate, 5 -cleftcrown; segments awned. Anth. bearded on the back; pollinia dilated at the apex and united to the corpuscles of the stigma, solitary, composed of four confluent grains.

190. GONOLOBUS. Cor. rotate, deeply 5-parted. Staminial crozan (lepanthium or nectary) scutelliform, pentangular. Anth. opening transversely, terminated by a membrane. Pollinia 5-pairs, not separating into grains. Stig. depressed. Seeds comose. 
191. ASCLEPIAS. Cal. small, 5-parted. Cor. rotate, 5-parted, mostly reflexed. Staminial crown sim. ple, 5-leaved; leaflets opposite the anthers, with a subulate averted process at the base. Sig. with the 5 angles (corpuscles) opening by longitudinal chinks. Pollinia 5 distinct pairs.

$$
\text { * Fruit capsular. }
$$

192. GENTIANA. Cal. 4-5 parted, or cleft. Cor. tubular at the base, campanulate; border $4-5$ cleft; segments ciliate or entire, spreading, erect, or connivent. Stam. included. Styles, 0 , or very short; stig. 2. Caps. 1-celled, 2-valved; recept. 2, longitudinal.

193. CUSCUTA. Cal. $4-5$ cleft. Cor. $4-5$ cleft, subcampanulate, marcescent. Caps 2-celled, opening all round transversely; cells 2 -seederl.

\section{B. Corolla 5-petalled, inferior.}

194. HEUCHERA. Cal. 5-cleft. Pet. 5, small, inserted into the margin of the calyx. Caps. birostrate, 2-celied, many-seeded.

C. Corolla 5-petalled, superior.

195. PANAX. Polygamous. Flowers in a simple umbel. Perfect. Cal. 5-toothed. Pet. 5. Styles 2-3. Berry subcordate, 2-3-seeded. STAminif. Cal. entire.

D. Flowers incomplete.

196. ATRIPLEX. Polgamous. Penfect rl. Cal. 5-parted, inferior. Cor. 0. Stam. 5. Style 2parted. Fruit depressed. Pistinif fl. Cal. 2-parted. Stam. 0. Fruit compressed. Seed ventical.

197. CHENOPODIUM. Cal. 5-parted, obtusely pentangular. Cor. 0. Style deeply bifid. Seed lenticular, horizontal, partly covered by the closing calyx.

198. SALSOLA. Cal. 5-parted. Cor. 0. Style bifid. Seed 1, horizontal, covered by the connivent ca. lyx. Embryo cochleate. 
199. ULMUS. Cal. campanulate, 4-5-cleft. Cor. 0. Samara compressed, with a broad membranaceous border.

200. CELTIS. Polygamous. Perfect FL. Cal. inferior 5-parted. Cor. 0. Styles thick, divaricate. Drupe 1-seaded, Staninif. Cal., 6-parted. Stam. 6.

\section{E. UHBELLIFER IE. \\ ๙. Umbels subcapitate.}

201. ERYNGIUM. Flowers capitate. Recept. paleaceous. Involucrum many-leaved, suhspinous. Pet. rellexed.

202. SANICULA. Umb. nearly simple, capitate. In. voluc. few-flowered. Fl. polygamous. Cal. 5 . parted, persistent. Fruit oblong, solid, not rib. bed, armed with uncinate bristles.

b. Unubels imperfect. Involucrum obsolcte or 0. (Leaves confluent with the petiole, subsimple.)

?03. HYDROCOTYLE. Umbel simple. Fruit with the back and commissure narrow, laterally compressed, subrotund, 3-ribbed, generally with reticulate reins.

¿. Uimbel perfect. Universul involverum, and sometimes the partial anting. Fruit orate, solid, 5 costute. (Leaves decompound.)

204. SISON. Fruit ovate, solid, 5-ribbed on the back; intervals conrex; sides contracted; raphis excavated.

305. CNIDIUM. Iniolucr. 1-leaved, or 0. Fruit ovate. solid. Ribs 5, acute, somewhat winged; intervols sulcate, striate.

1. Unibels perfect. Involucrum generally reanting. Fruit corticute, solid or compressed, utriculatc.

206. SMYRNIUM. Involucr. few-leaved, or 0. Fruit solid, ovate; cortex black, 3-ribbed. Sides contracted from the commissure. Albumen white.

207. CICUTA. Inoolur. almost wanting. Fruit ovate. solid, 5-riburd; interials promineut. 


\section{e. Fruit hispid.}

908. DAUCUS. Involucr. pinnatifid. Fl. somewhat radiant; those of the disk abortive. Fruit oblong, with 5 aculeate ribs; intervals angular, hispid.

f. Fruit pyramidal, rostratc. Involucrum 0 .

209. MYRRHIS. Fruit crowned with the pistils; rib: 5 , acute.

g. Froit subovate, costate, sulcate. Universal and partial involucra various.

210. SIUM. Fruit somewhat prismatic, with 5 obtusr. ribs ; intervals subconvex. Involucr. entire.

11. CONIUM. Fruit ovate, solid, with 5 obtuse ribs. crenulate when immature; intervals flat. Umbel with universal and partial involucra.

21:. LIGUSTICUM. Fruit ovate-oblong, with 5 acute ribs; intervals sulcate. Universal and partial involucra various.

11. Fruit compressed, flat, often rvinged. Involucra various.

213. HERACLEUM. Fruit with a membranaceous margin; ribs 3, dorsal, obtuse ; intervals and commissure with clavate spots. $F$. subradiant. Involucr. 0.

214. PASTINACA. Fruit oval, margined; ribs absolete; intervals striate; comrissures 2 . Universal and partial involucra 0 .

215. ANGELICA. Fruit subcompressed, with 3 acute winged ribs; intervals sulcate; margin membra naceous.

216. THAPSIA. Fruit narrow, slightly compressed, nearly ribless, with 2 dorsal and marginal winge. Involucra 0.

\section{TRIGYNIA.}

* Flowers superior.

¿17. VIBURNUM. Cal. minute, 5-toothed, superior Cor. 5-cleft ; lobes spreading or reflexed. Stigrmas nearly sessile. Berry l-seoder. 
13. SAMBUCUS. Cal. minute, 5-cleft. Cor. subrotate, 5-cleft. Stigmas minute, sessile. Derry globose, 1-celled, 3-seeded.

$$
\text { * Flowers inferior. }
$$

219. RHUS. Cal. 5-parted. Pet. 5. Berry small, suliglobose, with one bony seed.

220. STAPHYLEA. Cal. 5-parted, coloured. Petals 5 , inserted upon the margin of a glandular, pentangular disk. Caps. 2-3, inflated, partly united. Seeds 1-2 in each capsule, subglobose. with a lateral cicatrix.

\section{TETRAGYNIA.}

221. PARNASSIA. Cal. 5-parted, persistent. Pet. 5: inferior. Nect. of 5 scales, inserted into the claws of the petals, fringed with capitate cilix. Stig. 4, sessile. Caps. 1-celled, 4-valved; valves bearing incomplete dissepiments in the middle. Seeds membranaceously margined.

\section{PEN'IAGYNIA.}

222. ARALIA. Flowers umbelled. Cal. 5-toothed, superior. Pet. 5. Stig. sessile, subglobose. Bcrry 5-celled, 5-seeded.

-23. ST $\Lambda$ TICE. Cal. inferior, 1-leaved, entire, plaited, scarious. Pet. 5. Caps. 1-seeded, without valves. covered with the persistent calyx.

224. LINUM. Cal. deeply 5-parted, persistent. Pet. 5, unguiculate. Fil. united at the base. Caps. superior, subglobose, 10-valved, 10-celled. Seed: solitary, ovate, compressed.

325. SIBBALDIA. Cal. 10-cleft, with the alternate segments narrower. Pet. 5, inserted in the calyx. Styles proceeding laterally from the germen. Sceds 5, clustered in the bottom of the calyx.

\section{HEXIGYNIA.}

320. DROSERA. Cul, deeply 5-cleft, persistent. Pel. 5. Anth. adnate. Styles 6. Caps. superior. of relled, 3-5-valved, many-seeded. 


\section{POLYGYNYA.}

297. AANTHORHIZA. C'ul. 0. Pet. 5. Niect. 5, obovate, pedicellate. Caps. 5-8, 1-seeded. lialf 2-ralved.

\section{MIONOGYNIA.}

134. PULIIONARIA. Lung-wort. Borasinea.

P. virginica: smooth; stem erect; calyx much shorter than the tube of the corolla ; radical leaves obovate-oblong, obtuse; stem leaves narrower.

HAB. Banks of rivers. May. 24. Leav. smooth, subglauc.; fl. in term. racem., large, blue.

135. LITHOSPERHUM. Gromwell. Boraginea.

1. L. arvense: stern erect, branched; leaves linearlanceolate, rather acute, veinless, hairy; calyx nearly as long as the corolla, with spreading seg. ments; seeds rugose.

HAB. Fields. Apr.-Hay. ○. Hispidly pilose, 8$12 \mathrm{in.} \mathrm{high;} \mathrm{fl.} \mathrm{solitary,} \mathrm{axill.,} \mathrm{small,} \mathrm{white.}$

2. L. officinale: stem erect, much branched; leaves lanceolate, acute, nerved, scabrous above, hairy beneath; calyx as long as the tube of the corollia; seeds smooth.

HAB. Massacluusetts. May. 4. Stem. $2 f t$. hight scabr.; fl. axill., solit., piedicell., pule yellow.

3. L. maritimum: very smooth; stem procumbent, branched ; leaves oval, spathulate, fleshy ; corolli scarcely twice the length of the calyx.

HAB. Sea shores. July. 4. Stem diffuse; pedunc. lat, 1-лl.; cor. subcampan., blue.

t. L. canescens: stem erect, simple, villous; leaves oblong, obtuse, sillky above, subvillose beneath; tube of the corolla as long again as the calyx.

HAB. Fairfield, New-York. June. 4. Ruot large. red; fl. subterm., bright orange.

j. L. denticulatum: stem erect; leaves nervose, subglabrous, acute; margin scabrous, with minute teeth; radical ones ovate; those on the stem oblong; segments of the calyx denticulate on the mar. gin. 
HAB. New-York. June. 4. Stem. 6-10 in.high; pedunc. many-fl.; cor. purple.

1j. L. hirtum : plant hirsute; floral leaves ovate; stem. leaves oblong; calyx with long, lanceolate segments.

HAB. Pennsylvania. 4. Stem 8-12 in. high: $f$. in a term. raceme, orange.

\section{ONOSMODIUM. Boraginece.}

1. O. hispidum: plant hispid; leaves obovate-lancenlate, papillose-punctate; segments of the corolla subulite.

HAB. Dry hills. Aug. 4. Steni 1-2 ft. high; leav. subtriply nerved; fl. in leufy racemes, white.

2. O. molle: whole plant white villose ; leaves oblongoval, somewhat triply nerved; segments of the corolla semi-oval

HAB. Western countries. 4. Resembles the preceding, but has broader segments to the corolla.

\section{ECHIUM. Viper's bugloss. Boraginec.}

F. vulgare: stem tuberculite hispid; lcaves linearlanceolate, hispid; spikes lateral; stamens longer than the corolla.

$\mathrm{HAB}$ Hill sides. May-July. ot. Stem 2-3 $f t$ high; leav. very hispid; spik. secund, recurved; $A$. blue.

\section{MYOSOTIS. Scorpion-grass. Boragince.}

1. M. pulustris : leaves lanceolite, a little scabrous with appressed hairs; peduncles divergent when in fruit. twice as lung as the 5-toothed spreading calyx; border of the corollit flat, ionger than the tube.

IIAB. Ditches and rivulets. Hay-July. 4. Stem a ft. high, slend.; racemes long; $f l$. small, bright blue.

$\because$ M. aricnsis: leaves oblong-lanceolate, hairy ; racemes long; pedicels short, sprearling when in fruit; calys 5-cleft, closed; limb of the coroll: crect-sprearling, alsout as long as the tube.

IIAB. Sindy woods. June. $\odot$. Plunt gray, simp. or liranch.. 1-i) in. high; $A$. small, white. 


\section{LYCOPSIS. Small Bugloss. Borngine«.}

L. aroensis: leaves lanceolate, semiamplexicaul, te. pand-denticulate, very hispid; racemes leafy ; calyx erect.

HAB. Sandy fields. July. ○. Very hispid; fl. blue; seeds reticulate.

140. CYNOGLOSSUM. Hound's tongue. Boraginex.

1. C. officincle: lcaves broad-lanceolate, attenuate at thic base, sessile, downy; stamens included.

HAB. Road sides. June-July. ô. Dull green, $1 \frac{1}{2}-2$ ft. high ; $f$. panic., purp.-red; seeds rough.

$\therefore$ C. virginicum: leaves oval-oblong, acute, hairy; upper ones cordate and amplexicaul at the base; corymb on a long, naked peduncle.

IIIB. Moist wouds. May. 4. Slem $2-3 \mathrm{ft}$. high. retrorsely hairy; leazes lurge; $\Re$. Ulue and while.

\section{ROCH:LLI. Boragineu.}

3. J. Luppula: stem brarched above; leaves lancealate, with incumbent hairs; corolla longer than the: calyx; border erect-spreading; seeds with 2 row: of hooked prickles on the margin.

HAB. Road-sides, Sic. July-Aug. ๑. Stem panic. above, $1 \mathrm{ft}$. hight fluwers minute, blue.

2. R. virginiuna: leaves oblong-lanceolate, acuminate. sciabrous above; racemes divaricate; flowers nodding when in fruit; fruit densely covered with hovised prichles.

HAE. Rocky hills. July. of. Stem 2 ft. high, huiry; leuves large; fl. very smali, wilite.

\section{4\%. ANAGALLIS. Pimpernel. Liysinachice.}

1. aruensis: stem procumbent ; leaves ovate, sessile, dotted beneath; segrients of the corolla dilated, crenate-glandular.

HAB. Sandy fieli.s, Lc. June-July. ○. Stemi quadrang.; lear. oppos.; fi. solitary, axill. scarlet.

143. LYSIMACIIA. Loosestrife. Lysimachic.

1. L. stricta: raceme terminal, very long, lax; teave: opposite, linceolate, sessilc. 
HAB. Low grounds. July-Aug. 4. Very smooth; 18 in. high; axils often bulbif.; fl. yell.

2. L. quadrifolia : leaves verticillate, in fours and fives, ovate-lanceslate, acuminate ; peduncles axillary, 1 . flowered, by fours; segments of the corolla oval. entire, often obtuse.

HAB. Low grounds. June-July. 4. Stem 12-18 in. high, a little hairy; leav. punct. ; fl. yell.; stam. uneq.

3. L. ciliata: stem nearly smooth; leaves opposite, on long petioles, subcordate-ovate, acuminate; petioles ciliate ; peduncles subgeminate ; flowers nodding.

IIAB. Low grounds. July. 4. Stem 2-3 ft. high; leav. large; stam. nearly equal, with intermed. teetit.

4. L. hybrida: stem smooth ; leaves opposite, petiolate, lanceolate, acute at each extremity ; petioles ciliate : llowers nodding; peduncles axillary; corolla crenulate.

HAB. Swamps. July-Aug. 4. $18 \mathrm{in.} \mathrm{high;} \mathrm{inf.}$ leav. ovate-lun.; stam. nearly equal, with intermed. teeth.

3. L. longifulia: stem branched, smooth; leaves opposite, sessile, linear, very lung; upper ones in fours : peduncles by fours, subterminal, 1 -flowered; segments of the corolla ovate, acuminate, serrulate.

HAB. Wet woods. 4. Stem 2-3 ft. high; leav. 2 lin. broad, flat; floral ones verticill.; stam. subequal.

v. L. capitata: stem subsimple, punctate ; leaves opposite, scssile, broad-lanceolate, punctate; peduncles axillary, elongated; flowers in dense subglobose heads, 6-7-parted.

HAB. Swamps. Junc. H. Stem $18 \mathrm{in}$. high, terete: leav. punct. ; heads pedunc. ; stam. 6-7, much exserted.

7. L. revoluta: stem quadrangular, branched; leaves opposite, sessile, long-linear; margin revolute; pcduncles $\mathbf{1}$-flowered, subterminal, nodding.

IIAB. Wet calcareous rocks. Aurg. 4 Verysmoath, 12-18 in. high; leaves $3 \mathrm{in.}$ long, a lin. broad: anth. zery lurge.

14. PRIMULal. Primrose. Lysiniachior.

P. forinose 3. americana: leaves cubovate spathu. 
late, repandly crenite-dentate, pulverulent beneath; umbel many-flowered; peduncles spreading; border of the corolla flat, as long as the tube. with obtuse, obcordate segments.

IIAB. Shores of lakes. 4. Leaves all rad. with " yellowish-green powder beneath; $f$. pale purp.

\section{DODECATHEON. American Cowslip.} Lysimachio.

1. D. meaaia: leaves oblong-oval, repandly toothed; umbel many flowered, lax; bracts oval.

HAB. Rocky river siles. Hay. 4. Leav. rad., 4 in. long; scape 8-10 in. long; fl. nodding, purp.

$\therefore$ D. integrifolium: leaves oblong-oval, subspathulate; umbel few-flowered, straight; bracts linear.

IIAB. Mountains. 4. Fl. pale blue, smaller than the preceding.

146. MENYANTHES. Buck-bean. Gentianc.

II. trifoliata: leaves ternate.

IIAB. Swamps. May. 4. Stern a span high; leaflets ócrate; fl. reddish-rohite, in a pyramidal raceme.

\section{VILLARSIA. Gentiance.}

$b^{r}$. lacunosa: leaves reniform, subpeltate, slightiy crenate, lacunose beneath; petioles bearing the flovers; corolla smonth.

IAB. In ponds, floating. Aus. 4. Petioles rery long: leav. 1 is. long; fl. subumbellate, white.

14. HOTTONIA. Water-feather. Lysimaclia.

H. inflata: scape articulinte, with the internodes and lower part inflated; flowers verticillate, pedunculate.

UAB. Swamps, -subaquatic. 4. Sten thick; lear. pectinate; fl. white.

119. SAMOLUS. Brookweed. Lysimachice.

S. valcrandi: stem erect; leares obovate ; raceme: elongated, many-ilowered.

IIAB. Wet places. June-Oct. 4. 8-10 in. highi. amnoth: ji. miaule, white. 


\section{SABBATIA. Gentiance.}

1. S. campanulata : leaves sublinear; calyx as long asthe corolla.

HAB. Wet pastures. A foot high, terete; fl. subsolitary, purp.; seg. of cal. subulate.

2. S. stellaris: stem terete; branches dichotomous; elongated, 1-flowered; leaves lanceolate, acute ; segments of the calyx subulate, half as long as the corolla; segments of the corolla obovate.

HAB. Salt-marshes. Aug. $\tilde{\delta}$. A foot high, subangu!ar; fl. rose-col., with a yell. centre.

3. S. angularis: stem quadrangular, somewhat winged: leaves ovate, amplexicaul; peduncles elongated, corymbed; segments of the calyx lanceolate, mucl shorter than the corolla.

HAB. Moist meadows. Aug. $\odot$ and 8 . I foot high, branched; $f$. rose-col.; seg. obovate.

1. S. calycosa: stem erect, few-flowered; leaves oljlong; flowers solitary, 7-9-parted; calyx foliaceous, longer than the corolla.

HAB. Wet meadows. \&? A foot high, very leafy; segments of the cal. lanceol.; fl. rose-col.

j. S. chloroides: stem weak; leaves lanceolate, crect; branches 1-flowered; flowers 7-12-parted; segments of the calyx linear, shorter than the corollit. HAB. Brackish bog-meadows. Aug. of: Stcm 2ft. high; $f$. mostly 9-parted, 2 in. in diancter. bright rose-colour.

j. S. corymbosa: leaves ovate, closely sessile; sten acutely angled; flowers corymbed; segments of the calyx subulate, much shorter than the corolla.

HAB. Swamps. Aug.-Sept. 4. A foot high ; co. rymb. fere-flo; fl. white, sometimes 4-parted.

151. HYDROPHYLLUN. Water-leaf. Boraginco.

1. H. virginicum : stem nearly smooth; leares pinna tifid and pinnate; segments oval-lanceolate, with deep serratures; fascicles of tlowers conglomerate ; peduncles longer than the petioles.

HAB. Woods. May-June. 4. Stem $1 \frac{1}{2} f_{\text {t. high }}$;

leav. 2-3. large; cor. white, with purp. vcins.

¿. H. canadense: somewhat hairy; leares angelarly 
somewhat 5 lobed, remotely serrate; pedunclu: shorter than the petioles.

HAB. Woods and mountains. June. 4. Leav. on long petiol., suhcordate; $f$. white and purp.

3. H. appendiculatum: very hairy ; radical leaves subpinnatifid; cauline ones angularly lobed; sinuses of the calyx with minute oval ippendages.

HAB. Shady wet rocks. May. of Almost hispid; fl. subpanic., on short pedunc., blue.

\section{PHACELIA. Boraginece.}

1. P. bipinnatifida: stem erect; leaves pinnatifid; segments incisely lobed; racemes mostly bifid, oblong, many-flowered; divisions of the corolla entire.

HAB. Mountainous regions. May-June. \& ? Hairy; fl. in term. subsecund racemes, blue.

$\therefore$ P. fimbriata: stem ascending, hairy; leaves with undivided lobes; segments of the corolla fimbriate. HAB. Western countries. 4, $\odot$. Racemes short, at first revolute; cal. hispid; fl. blue.

3. P. parviftora: stem diffuse, pubescent; leaves subsessile, pinuatifid; neguents oblong, rather ohtuse, entire ; racemes solitary ; perlicels short ; segments of the corolla round, very entire.

IAB. Pennsylvania. May. $\odot$. Stem 6-8 in. high, slender; $f$. pale blue or white; stam. short.

153. SPIGELIA. Carolina Pink-root. Gentiance.

S. marilandica: stem simple, quadrangular; leares all opposite, sessile, ovate-lanceolate.

IIAB. Woods. June. 4. A foot high; fl. fusiform. crimson, in a secund terminal raceme.

154. TERBASCUM. Mullein. Solanece.

1. V. Thapsus: leaves decurrent, woolly on both sides; raceme spiked, dense; two of the stamens glabrous. HAB. Fields, road-sides, \&c. June-Aug. 8. Stent 3 -6 ft. high; fl. bright yellow in very long spikes.

2. V. Blattaria: leaves amplexicaul, oblong, smooth, serrate; peduncles 1 -flowered, solitary

IAB. Old fields, \&c. June-July. of. $2 \mathrm{ft}$. high, ang.; raceme leafy; pericels 1 in. long; stam. uneq.

\%. alba: leaves toothed; flowers white.

3. lutea: leaves doubly serrate; flowers yellow. 
;. V. Lychnitis : leaves oblong-cuneiform, naked above; stem angular. panicled.

HAB. River banks. of. Leaves very woolly bencath : f. pale yellow.

\section{NICOTIANA. Tobacco. Solanece.}

N. rustica: stem terete ; leaves petiolate, ovate, very entire; tube of the corolla cylindrical, longer than the calyx; segments round.

HAB. Borders of lakes. $\odot$ Viscosc-pubes. $1 \frac{1}{2} \mathrm{ft}$ high; fl. in a term. pan., dull greenish-yellow. §

\section{HYOSCYAMUS. Henbane. Solanece.}

H. niger : leaves amplexicaul, sinuate; flowers sub. sessile ; corolla reticulate.

IIAB. Road-sides and rubbish. June. $\odot, \delta$. His pidly-pilose, fotid; $f l$ in recurved spikes, dingy yel low. $\$$

157. CONVOLVULUS. Bind weed. Convolvuli,

1. C. arvensis: stem climbing; leaves sagittate, with the lobes acute; peduncles mostly 1 -flowered; bracts acute, remote from the flowers.

IIAB. Fields. 24. June. Stem a little hairy: lear. obtuse; fl. small, rehite; stig. linear.

$\therefore$ C. Sepium: stem climbing; leaves sagittate; very acute; lobes truncate; bracts close to the flower. acute, longer than the calyx ; peduncles quadrangular, 1 -flowered.

HAB. Hedges and low grounds. June-July. 24. Lcav. cordate-sugittate; pedunc. long; $f$. large, white.

3. C. panduratus: stem climbing ; leaves cordate, entire or lobed, panduriform; peduncles long; flowere fisciculate; calyx smootb; corolla tubular-campanulate.

IIAB. Sandy fields. 4. Aug. Ront very large; stem invstly trailing; pedunc. $1-4 \mu_{0}$; tube of the cor. purp.

1 C. spithanceus: stem erect or trailing; leaves oval or oblong, subcordate, pubescent ; peduncles 1flowered, generally longer than the leaves.

HAB. Dry woods. June. 4. Stem 1-3 ft. lone, pubes.; lecu. obtuse or acum.; bracts large. 


\section{IPOMFA. Convolvuli.}

1. 1. purpurea : leaves cordate, entire ; peduncles $2-$ 3-flowered; pedicels incrassated, nodding; capsule smooth.

IIAB. Cultivated grounds and waste places. JulyAug. $\odot$. Stem hairy, twining; cor. large, blue and purp.

2. 1. lacunosa: stem smooth; leares cordate, acuminate, angular at the base ; peduncles short, generally 1 -flowered; calyx hairy; corolla tubular, short ; capsule hairy.

HAB. Pennsylvania. Aug.-Sept. ○. Flowers white, with a purp. rim.

3. I. Nil: stem hairy; leaves cordate, 3-lobed; peduncles short, 1-3-flowered; calyx very vi!lous ; segments acuminate.

HAB. Hedges and gardens. ๑. Leaves acım., 3.

- lobed when old; cor. infund., rhite at base.

\section{PHLOX. Polemonia.}

1. P. paniculata: stem erect, smooth; leares lanceolate, flat; corymb paniculate; segments of the corolla rounded.

MAB. Moist meadows. 24. June. Stem $2-3 \mathrm{ft}$. high; leav. acum. ; cor. purp.; tube pubes.

$\therefore$ P. pyramidalis: erect, smooth; stem scabrous; leaves cordate-ovate, acuminate; panicle fastigiate, pyramidal ; segments of the corolla cuneate ; truncate; teeth of the caly 8 somewhat erect, lanceo. late, acute.

HAB. Mountain meadows. Aug. 4. Stem 2-3 ft. high, spotted with red!; fl. purp.; teeth of the cal. short.

1. P. maculata: erect; stem scabrous and spotted; leaves oblong-lanceolate, smooth, scabrous on the margin; panicle oblong, many-tlowered; scgments of the corolla rounded; teeth of the cal! $x$ acute, recurred.

HAB. Rich moist meadows. June. 2f. Stem $Q_{\varepsilon}^{I}$ ft. high, simp.; upper leav. ovate; $f$. pale purp.

3. suaveolens: stem not spotted; corolla white.

‥ P. aristata: stem erect, weak, viscid-pubescent; leares linear-linceolate; panirle lax, fistiginte: 
segments of the corolla obovate; tube curved, pubescent; teeth of the calyx very long, subulate. HAB. Wet meadows. June. 4. Stem $1 \frac{1}{2} \mathrm{ft}$. high, villose-pubes.; teeth of the cal. aristate; cor. pale purp. or white.

5. P. divaricata: stem low, pubescent, decumbent; leaves oval-lanceolate; upper ones alternate; branches divaricate, loose, few-flowered; segments of the corolla obcordate; teeth of the calyx subulate-linear.

HAB. Rocky bauks. July. 4. Stems numerous, $9-12$ in. high ; $f$. in term. pan., blue ; cal. erect.

6. P. reptans: pubescent, with creeping stolons; radical leaves spathulate-obovate; stem-leaves oval. lanceolate ; corymb few-flowered, divaricate ; segments of the corolla obovate; teeth of the calyx subulate, reflexed.

HAB. Mountains. June. ì. Stem 6-8 in. high, erect ; corymb 1-6- $f$. ; cal. pubes. ; $f$. blue.

i. P. subulata: cespitose, pubescent; leaves linearsubulate, rigid, ciliate; corymb few-flowered; segments of the corolla cuneate, emarginate ; calyx with subulate teeth about as long as the tube of the corolla.

IIAB. Rocky hills. April-May. 4. Procumb., branching, 3-4 in. high; lower leav. fascic.; $f$. pink.

3. P. setacea : cespitose, pubescent ; leaves fasciculate, subulate, ciliate, rigid; flowers few, terminal, somewhat umbelled; segments of the corolla cuneate, emarginate; teeth of the calyx subulate, much shorter than the tube of the corolla.

IIAB. Rocks and sandy hills. May. 24. Stem assurgent; leav. longer than No. 7.; fl. pale rose-col.

360. POLEMONIUM. Jacob's-ladiler. Polcmonia.

P. reptans: leaves pinnate; leallets about 7 ; 1lowers terminal, nodding.

HAB. Fertile hills. May \& Root crecping; stem erect, 1 ft. ligh; fl. subcorymb., blue. 


\section{DIAPENSIA. Convolvuli.}

1. D. lapponica: cespitose; leaves spathulate, smooth; flowers pedunculate; anthers simple.

HAB. High mountains. June. 4. Evergreen, leav. much crowded, a little recurv.; fl. term., white.

2. D. barbulata : creeping; leaves cuneate-lanceolate, pubescent at the base; flowers sessile; anthers beaked at the base.

HAB. Pine barrens and high mountains. MayJune. Evergreen, forming mats ; fl. solit., term., white.

162. DATURA. Thorn-aprle. Solanea.

D. Stramonium : capsule spiny, erect; leaves ovate, smooth, angular-dentate.

HAB. Waste-places. July-Sept. ○. Stem 2-7 $f t$. high, dichot., green; $f$. solitary, axill., white.

B. Tatula: stem and flowers purple.

163. AZALEA. Rhododendra.

A. procumbens.

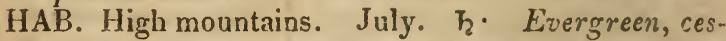
pitose, 3-4 in. long; leav. oppos., ellipt. ; $f$. in smail term. umb., rose-col.

164. PHYSALIS. Ground-cherry. Solanece.

1. P. obscura: pubescent; stems prostrate, divaricate : leaves broad-cordate, subsolitary, unequally and coarsely toothed; flowers solitary, nodding; calyx very hairy.

HAB. Woods and hills. Ang. - ๑. Stcm forked: leav. on long petioles; $f$. pedunc., dull ycll.

2. P. vicosa: leares by pairs, subcordate-oval, repanil. obtuse, subtomentose; stem herbaceous, paniculate above; fruit bearing calyx pubescent.

HAB. Road sides, \&c. $\odot$. Stem ercet, $2-S$ fi. high, dichot.; fl. ycll., spotted at the base.

3. P. pennsylvanica: leaves ovate, subrepand, obtuse. ncarly naked; stem herbaceous, branched; peduncles solitary, a little longer than the petioles.

HAB. Roarl sides and rubbish. July-Sept. 4. Erect or procumb., 1 ft. high; $f$. yell.; not spotte 
k. P. lanceolata: leaves geminate, oval-lanceolate entire, narrowed at the base into a petiole; stem herbaceous, dichotomous; calyx villous.

HAB. Pennsylvania. 4 . Stem 1-2 ft. high. densely pubes. ; fl. pale yell., spotted.

165. SOLANUM. Nightshade. Solanea.

1. S. Dulcamara: stem fruticose, flexuous, withoui thorns ; leaves ovate-cordate ; superior ones hastate; corymbs opposite the leaves.

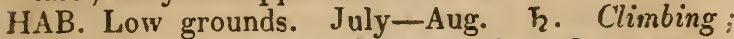
fl. in lateral clusters, violet; berries red.

2. S. nigrum d. virginianum: stem herbaceous, without thorns, angular, toothed; leaves ovate, obtusely toothed and waved; flowers subumbelled.

HAB. Old fields, \&c. July-Aug. ○ . Stem diffuse, slightly winged; $f$. white; berries black.

3. S. carolinense : stem annual, aculeate; leaves ovate. oblong, tomentose, hastate and sinuate-angulạr. acuminate ; racemes simple, lax.

HAB. Fields. June. 4 . Erect, branched, $1 \mathrm{ft}$. ligh, armed with yell. prickles; $f$. white or pals llue; berries yell.

166. CAMPANULA. Bell-flower. Campanula.

1. C. rotundifolia: glabrous; radical leaves reniform. cordate, crenate; cauline ones linear, entire; panicle lax, few-flowered.

HAB. Rocky river banks. June. 2f. Root creeping ; stem erect ; $f$. feiv, drooping, blue.

2. C. amplexicaulis: stem simple, erect; leaves cordate, crenate, amplexicaul ; flowers axillary, sessile, glomerate.

HAB. Fields and dry hills. May-July, ○. Stem 8-12 in. high, hispid; leav. veined; fl. purp.

3. C. americana: leaves ovate-lanceolate, much acuminate, membranaceous, uncinately serrate; lower ones subcordate, with the petioles ciliate ; flowers subsolitary, nearly sessile, in a terminal leafy raceme ; corolla subrotate; style exserted.

HAB. Wet rocks. Aug. 2f. Stem $2 \mathrm{ft}$. high, simp.; pedicels short, clavate; fl. blue, flat. 
4. C. acuminata: nearly smooth; stem straight, erect, leaves lanceolate, attenuated to a long point, subserrate; spike fasciculate, many-flowered; corolla subrotate.

HAB. High mountains. 24. Stem erect, $2-3 \mathrm{ft}$. high; leav. remotely serrate; $f$. by threes, blue.

5. C. aparanoicies: stem slender, branched, acutely subtriangular; angles, with the margin and nerves of the leaves, retrorsely aculeate ; leaves linearlanceolate, smooth above ; perluncles few, terminal, filiform, flexuous, 1-flowered.

HAB. Wet meadows. June-Aug. ○. Erect or diffuse, $1 \mathrm{ft}$. high; leav. dentic.; fl. small, white.

\section{LOBELIA. Lobeliucece.}

ᄂ. L. Dortmanna: leaves linear, fleshy, 2-celled, obtuse ; sc:tpe nearly naked; flowers racemed, remote. HAB. Swamps, - subaquatic. July. 24. Leaves immersed: radical ones spreading; scape long, $3-4-f$; cor. blue.

2. L. Kainii: whole plant smooth; stem slender, erect, branched ; leaves linear, remotely denticulate; ratical ones spathulate; raceme lax, fewflowered, leafy; peduncles longer than the fruit, with 2 minute bracts near the flower; capsule attenuate at the base.

HAB. Rocky bınks of rivers. July-Aug. \& ? Stem 12-18 in. high; pedunc. 1 ì. long; fl. pale blue.

3. L. Nuttallii : stem erect, minutely scabrous, simple, or with filiform branches; leaves oblong-linear, denticulate; flowers in slender ricemes, distant; peduncles coloured, shorter than the flower, with minute bracts near the base; capsule obtuse be. low.

HAB. Borders of sandy swamps. Aug. \&. Stem slender, $2 \mathrm{ft}$. high; seg. of the cal. longer than the caps. ; fl. pale blue.

1. L. Claytoniana: stem erect, simple, pubescent: leaves oblong, pubescent, obtuse, nearly entire; radical ones spathulate; raceme virgate, naked; 
segments of the calyx subulate; nearly as long as the tube of the corolla.

HAB. Dry woods and fields. Aug. 4. Stem $1 \frac{1}{2}-2$ ft. high; rad. leav. nearly entire; fl. pale blue.

j. L. puberula : pubescent; stem erect, simple; leaves oblong-oval, obtuse, repand-serrulate; spike secund, leafy at the base; segments of the calyx longer than the tube of the corolla, ciliate.

HAB. Mountains. Sept. 4. Stem 2 ft. high; rad. leav. obovate; sinuses of the cal. reflexed; $f$. large, blue.

6. L. siphilitica: stem erect, rather hairy; leaves ovate-lanceolate, unequally serrate ; raceme leafy ; calyx hairy, with the sinuses reflexed.

HAB. Wet mearlows. Sept. 4. Stem $1 \frac{1}{2}-2 \mathrm{ft}$. high; $f$. on short pedic., large, bright blue.

7. L. inflata: stem hairy, branched; leaves ovate-lanceolate; racemes leafy, somewhat paniculate ; capsules inflated.

HAB. Fields. Aug. ○. Acrid, a foot or more high ; leav. crenate; $f$. pedunc., small, pale blue.

3. L. Cardinalis : stem erect ; leaves oblong-lanceolate, serrate ; raceme secund, somewhat leafy ; stamens longer than the corolla.

IIAB. Wet grounds. July-Aug. 24. $2 \mathrm{ft}$. high, very smooth; fl. large, bright scarlet.

168. DIERVILLA. Caprifolia.

1). T'ournefortii.

IIAB. Rocky woods. June. h. Shrub 2-3 high, branched; leav. oppos., ovate, acum., serra ft. pedunc. axill., 2-3-fl.; cor. yell.

169. LONICERA. Honey-suckle. Caprifolia.

1. I. hirsuta: spikes verticillate, terminal, subcapitate, glandular-pubescent; leaves broad-ovate and obovate, pubescent and ciliate, glaucous beneath; upper ones connate-perfoliate, nearly smooth. HAB. Rocky woods. June h. Climbing; leav. decid., nearly sess., sometimes almosi smooth; $f$. yell., pubcs.; lerries orange, glandular-pubes. 
3. L. Aava: spikes verticillate, capitate, terminal ; corolla somewhat ringent; segments oblong, obtuse ; leaves ovate, glaucous beneath, with a cartilaginous margin ; upper ones connate-perfoliate.

HAB. Mountains. June-July. h. Climbing : leav. decid., smooth, veined; fl. bright yell.; tube hairy.

3. L. parviflora: spikes verticillate, capitate; leaves deciduous, glaucous beneath, all of them connateperfoliate; corolla ringent, gibbous at the base: filaments bearded.

- HAB. Rucky places. June. Ћ. Climbing; glaucous, leav. ovate or oblong; fl. yell.; berries red.

5. L. grata: flowers verticillate, terminal ; leaves perennial; obovate, glaucous beneath; upper ones connate-perfoliate.

HAB. Swanps and mountains. h. Climbing or erect; fl. inclining to scarlet, ringent; berries red.

5. L. sempervirens: spike somewhat naked, verticillate, terminal ; leaves oblong, glaucous beneath ; upper ones connate-perfoliate ; corolla nearly. equal, with the tube above ventricose.

HAB. Dry stony woods. May-Aug. h. Evergreen; leav. a little hairy above; fl. crimson; berries scarlet.

170. XYLOSTEUM. Caprifolia.

1. X. ciliatum: leares ovate and subcordate, ciliate; the younger ones villous beneath; tube of the corolla calcarate at the base, ventricose above: segments short, acute ; style exserted.

HAB. Mountains. May-June. Ћ. 3-4 ft. high: branch spreading; leav .on short pet.; fl. axill., yell.

3. X. villosum : berries coadunate; leaves oblong and aval, obtuse; the younger ones, with the corolla, villous; peduncles much shorter than the flower. HAB. Mountain swamps. Hay. ${ }_{2} .2-4 f t$. high; young branch. vill.; fl. axill., ycll.; berries red or purp.

171. SYMPHORIA. Peter's-wort. Caprifolia.

1 S. glomerata : racemes axillary, capitate, conglome. rate. 
HAB. Pennsylvania. Aug. h. 3-4 ft. high; bran. erect, pubes.; leav. oppos., ovate, entire; $f$. greenish-red, swall; berries purp.

3. S. racemosa: racemes terminal ; corolla bearded within.

HAB. Rocks. July. Ђ. 2-3 ft. high, branched; A. small, pale red; berries large rathite.

172. TRIOSTEUM. Fever-wort. Caprifolia.

T. perfoliatum: leaves oval, acuminate, abruptly narrowed at the base, connate, pubescent beneath ; axils 1 -3-Howered.

HAB. Rocky woods. June. 4. Stem 2-3 ft. high ; leav.large; fl. sess., dull purp.; berries orunge.

173. ITEA. Saxifragece.

1. virginica.

HAB. Swamps. June. h. 4-8 ft. high; leav, altern., lanceol., acum., serrul.; fl. in simp. term. rucemes, white, pubes. within.

17. IMPATIENS. Balsam. Papaveracece.

1. I. pallida : peduncles solitary, 3-4-flowered; nectary obtusely conic, dilated, shorter than the petals; spur recurved, very short ; flowers sparingly punctate ; leaves rhombic-ovate, mucronately toothed.

IIAB. Wet shady places. Aug. $\odot$. Stem $2 \mathrm{ft}$. high, terbder and succul.; leav. altern.; fl. iyell.; seeds ellip.

2. I. fulva: peduncles solitary, 3-4-flowered; nectary acutely conic, longer than the petals; spur resupinate, emarginate, nearly as long as the galea ; nowers with crowded spots; leaves rhombic-ovate, obtuse, mucronate toothed.

HAB. Wet shady places. Aug. ๑. Plant glauc. and diaph.; caps. 5-ang., 3-5-seeded; seeds prisinat.

17j. VIOLA. Violet. Violacere. * Stemless.

$\dagger$ Flowers blue.

1. V. pedate: leaves pedate, nearly smonth, about i- 
parted; segments linear-lanceolate, entire; stigma large, obliquely truncate, and perforate at the apex.

HAB. Rocky hills and dry woods. May. 4. Leav. with obtuse seg.; stip. ciliate; $f$. large, pale blue.

2. V. palirata: pubescent; leaves cordate, (rarely entire,) palmate, or hastate-lobed; lobes crenate and toothed, the middle one much the largest; 2 lateral petals bearded; stigma margined, depressed.

HAB. Low grounds. May. 4. Leaves never smooth; stip. ian.; fl. middle sized; stig. rostrate.

3. V. sagittata: leaves oblong, acute, cordate-sagittate, incised at the base; flowers inverted; inferior petal bearded, the rest bearded.

HAB. Hills and fields. April-May. 4. Leav. pubes. above; stip. lin.; fl. middle-sized, purp.; spur short.

ß. emarginata: leaves almost triangular, lacerately toothed near the base ; petals emarginate or bidentate.

HAB. Sandy fields and river-banks.

1. Y. ovata: leaves ovate, subcordate, crenate, often lacerately toothed at the base, pubescent on both sides; petiole margined; segments of the calyx oblong-lanceolate, hairy.

HAB. Dry hills. April-May. 4. Leav. gene. rally almost woolly; fl. middle-sized; stig. recurv.: rost.

3. V. cucullata: very smooth; leaves cordate, cucullate at the base, serrate; stipules linear; inferior and lateral petals bearded.

HAB. Wet meadows. April-May. 4. Leav. subrenif., on long petioles; beard of the pet. rigid.

6. V. obliqua: smooth; leaves cordate, acute, flat, crenate-serrate; flowers obliquely bent; lateral petals bearded; inferior ones acute, carinate; stipules lanceolate.

HAB. Wet meadows. 4. May. Scapelong, with minute bracts; Leard of the pctals globose-clavate.

7. V. villosa: leaves reniform-cordate, flat, very pubescent; segments of the calyx auriculate at the base ; stigma deflexed; margin cupuliform.

IIAB. Rocky hills. 4. May. Leav. spreading an the ground. purp. beneath : pet, oblong. 
3. cordata: leaves cordate, rather acute ; segments of the calyx scarcely produced at the base.

HAB. Hills and dry woods. April-May. 4. Leav. thickish, smooth below ; sinus small.

\section{$\dagger \dagger$ Flowers yellow.}

3. V. rotundifolia: leaves orhicular-ovate, cordate, slightly crenate, nearly smooth; sinus closed; petiole pubescent ; lateral pet:ls bearded; segments of the calyx obtuse; spur nearly obsolete.

HAB. Shady rocky woods. May. 4. Leav. ap. press. to the earth, on short pet.; pet. broad-ovate, striate.

$\dagger \dagger \dagger$ Flowers somewhat regular, small, white.

3. V. lanceslara: leaves very smooth, lanceolate, attenuated into a petiole at the hase, rather obtuse, subserrite; petals beardless.

HAB. Wet meadows. April-May. 4. Leav. 2 -4 in. long, very narrow ; fl. inodorous, veined.

10. V. acuta: leaves ovate lanceolate, smooth, abruptly decurrent at the base; bracts lancololate-linear; petals acute, of nerrly equal length, beardless.

HAB. Moist grounds. May. 4. Leav. rarely subcord.; pe!. ovate, lowest one veined.

11. V. primulafolin: leaves oblong, subcordate, abruptly decurrent on the petiole; nerves beneath, and the scape, subpubescent; lateral petals bearded.

HAB. Wet meadows. April-May. 4. Leav. more or less ob'use; $f$. odor'us; pet. obovate.

12. V. blanda: leaves broad-cordate, slightly pubescent above ; sinus rounderl; petiole smooth; flowers beardless.

HAB. Wet grounds. April-May. 4. Leav. nearly flat, meribrun., of ten subrenif.; $f$. oclorous.

13. V. clandestina: leaves orbicular, mather obtuse, smoothish, crenate-serrate, with glandular serratures; sinus closed, cordate; stolons floriferous; petals linear, scarcely longer than the calyx. 
HAB. Mountains. June-Sept. 4. Leaves large, thin; scape very short; $f$. concealed. A var. of No. 12 ?

\section{* Caulescent.}

14. V. canadensis: nearly smooth; leaves cordate, acuminate, serrate; peduncles shorter than the leares; petals oblong, narrow ; stipules ovatelanceolate, entire.

HAB. Moist rocky woods. May-July. 4. Siem 6-18 in. high, erect, simp.; fl. large, blue.

15. V. ochrolenca: stem somewhat erect, branched, smooih ; leaves round-cordate ; upper ones a littie acuminate ; stipules oblong-lanceolate, dentateciliate; stigma tubular, recurved, pubescent at the summit.

HAB. Wet grounds. May. 4. Stem 6-8 in. long; fl. yelloreish-rohite, large; pet. obtuse.

16. V. Niuhlenberghii : stem weak, assurgent; leaves reniform-cordate ; the upper ones a little acuminate, crenate-serrate, smooth ; stipules lanceolate, serrate-riliate; nectary produced; stigma tubular, rostrate.

HAB. Swamps. May. 4. Stem 6-10 in. long, branch. below ; bracts alternate; $f$. blue.

17. V. rostrata: smooth; stem diffuse, erect; leaves cordate, the upper ones acute, serrate; stipules lanceolate, serrate-ciliate; petals beardless; nectary longer than the corolla.

HAB. Wet rocky places. April-May. 4. Stem 6 in. high; pedunc. long; fl. large, blue; stig. subclavate.

18. V. pubescens : villous pubescent; stem erect, naked below ; leaves broad-cordate, toothed; stipules ovate, subdentate.

HAB. Dry stony woods. April-May. Stem simp., 6-8 in. high, bructeate; leav. 2-3; fl. yellow.

ß. eriocarpa: capsule densely villous.

19. V. hastata: smooth; stem simple, leafy above; leaves hastate, on very long petioles; stipules minute, ciliate-denticulate. 
HAB. Mountains. May, 4. Erect, 6-8 in. high; leav. acum.; fl. yell.; inf. pet. large, sub-3-lobed. 30. V. tenella: stem triquetrous ; leaves rotund-spathulate; superior ones lanceolate, entire; stipules large, pinnatifid.

HAB. Dry rocky hills. May. $\odot$. Stem 2-3 in. high; rad. leav. petiolate; fl. small, bluish-zohite.

21. V. cuncolor: stem straight, erect ; leaves cuneatelanceolate; stipules lanceolate-entire; peduncles very short, 2-3-flowered; petals connivent, emarginate; spur 0.

HAB. Wet shady rocks. April-May. 4. Stem simp., ang., $1 \mathrm{ft}$.high; leav. erect ; $f$. greenish, small.

176. CLAYTONIA. Portulacece.

C. virginica: leaves linear-lanceolate; petals obovate, retuse; leaves of the calyx somewhat acute; root tuberous.

HAB. Moist woods. April-May. 4. Smooth, erect or procurnb.; leav. fere, oppos.; fl. racem., rose-col. 3. latifolia: leaves ovate-lanceolate; leaflets of the calyx obtuse.

HAB. Mountainous districts.

177. CEANOTHUS. New-Jersey tea. Rhamni.

1. C. americanus : leaves ovate-oblong, serrate, triplynerred, tomentose-pubescent beneath; panicles axillary, on long peduncles.

HAB. Woods and copses. July. Ћ. Root large, red; stem 2-4 ft. high; leav. alt.; fl. minute, white.

?. C. herbaceus : leaves oval, subserrulate, smooth; panicle thyrsoid, axillary and terminal.

HAB. Rocky banks. Suffruticose; leaves as large as in the preceding.

178. EUONYMUS. Spindle-tree. Rhamni.

i. E. americanus: branches smooth, quadrangular; leaves subsessile, elliptical-lanceolate, smooth,acute, serrate; peduncles 3 -flowered, terete ; flowers 5 cleft; fruit verrucose-muricate.

HAB. Shady woods. June. Shrub 3-4 fi. high: bran. oppos., spread.: A. axill., greenish; fruil scarlet. 
3. sarmentosus: stem sarmentose.

HAB. Rocky moist forests. July. Subsempervirent:

$2 \mathrm{ft}$. high, acutely quadrung.

2. E. atropurpureus: leaves perfoliate, oblong-lanceolate, acuminate, serrate; peduncles livaricate, manyflowered; flowers 4-cleft ; fruit smooth.

HAB. Shady woods. June. Shrub $4-5 \mathrm{ft}$. high; pedunc. 3-6-fl.; pet.dark-purp.; fruit bright-red.

3. E. ohıvatus: stem prostrate, rootıng; surculi erect, obtusely quadrangular, with 4 elevated lines; leaves broar-ovate, obtuse, acute at the base, subsessile, acutely serrulate; peduncles 3 -flowered; stamens $4-5$.

HAB. Shidy fir swamps. June. leav. ofien dilated; fl. purplish-green.

179. CELASTRUS. Staff-tree. Rhamni.

C. scandens: stem climbing, unarmed; leaves oblong, acuminate, serrate; racemes terminal ; flowers diœcious.

HAB. Woods and thickets. June. Ћ. Leav. altern., stipui.; racem. few-fl.; pet.greenish-yel.; fruit red.

180. RHAMNUS. Buck-thorn. Rhamni.

1. R. alnifolius : unarmed; leaves oval, acuminate, serrulate, pubescent on the nerves beneath ; flowers diœcious; peduncles 1-flowered, aggregate; calyx acute; fruit turbinate.

HAB. Rocky hills. May. Shrub small; leav.altern.: fl. small, greenish, in axill. fascic.; fruit black.

2. R. catharticus: spikes terminal; flowers generally 4-cleft, polygamo-diœecious; leaves ovate; stem erect; berries 4 -seeded.

HAB. Mountains and woods. May. A small tree: leav. fascic. and oppos.; fl. clust., axill.; fruit black. ई ?

\section{VITIS. Vine. Vites.}

1. V. Labrusca: leaves broad-cordate, angularly sul)3 -lobed, cinereous-tomentose beneath; racemes small ; berries large.

HAB. IVoods and hedges. June-July. $h_{\text {. Leav. }}$ very large, at first ferrug. beneath; $f$. greenish: frutit purp. 
$\therefore$ V. vulpina: leaves cordate, acuminate, incisely toothed, smooth on both sides; racemes loose, many-flowered; berries small.

HAB. Woods and river-banks. June. $\zeta_{2}$. Leav $3-4$ in. hroad; veins a little pubes.; fruit sm., amber-col.

3. V. cestivalis : leaves broad-cordate, 3-5-lobed, ferruginous-tomentose beneath; sinuses rounded: racemes oblong; berries small.

HAB. Rocky river-banks. June. h. Leav. smoothish when old; fruit deep blue or purp.

ß. sinuata: leaves sinuate-palmate.

4. V. riparia: leaves unequally incis ely toothed, short 3-lobed, pubescent on the margin, nerves and petiole.

HAB. Gravelly shores. $\quad$. $F l$. very sweet scented.

182. CISSUS. Vites.

§. C. hederacea: stem climbing and rooting; leaves quinate-digitate, smooth; ledflets petiolate, oblong, acuminate, toothed; racemes cymose, dichotomous; nectary 0.

HAB. Woods. July. 万. Leav. on long pet.; $f$ greenish; ber. small, durk-blue, acid.

3. hirsutix: leaves pubescent on both sides; leaflet: ovate, acuminate, coarsely toother.

HAB Mountains.

2. C. Ampelopsis : leaves cordate, acuminate, toothed and angular; nerves beneath pubescent ; racemes twice bifid.

HAB. River banks and mountains. $\Upsilon_{2}$. Climbing; leav. petiol.; raceme ferw-fl.; ber. pale-red.

183. RIB.ES. Currant and Gooseberry. Grossularice.

* Steins without thorns.

2. R. floridum: unarmed; leaves punctate both sides, acutely 3-lobed, pubescent; racemes pendulous: calyx tubular; bracts longer than the pedicels.

HAB. Woods and hedges. h. April- "Tav. Shrul 3-4 ft. high; fl. greenish; ber, black. 
פ. R. albinervium : leaves short, acutely lobed, smooth. ish; nerves white; racemes recurved; berries smooth.

HAB. Mountains. April-May. そ. Fl. small, greenish yell.; ber. red.

3. R. trifidum: leaves moderately lobed, smooth above, pubescent beneath; racemes loose, pubescent; flowers rather flat, with the segments of the calyx subtrifid; petals spathulate, obtuse ; berries hairy. HAB. Mountains. April-May. h. Leav. with subacute lobes; pet. purp.; ber. red.

4. R. rigens : branches straight; leaves acutely lobed and dentate, reticulate-rugose, pubescent beneath ; racemes rather loose, many-flowered, becoming stiffly erect; berries hispid.

HAB. Mountains. May. Ђ. Leav. on long pet.; raceme 6-10-fl.; fl. purp.; ber. red.

5. R. prostratum: branches reclined-prostrate; leaves lobed, smoothish; the younger ones pubescent; branches somewhat erect; culyx rather flat; petals deltoid ; bracts minute; berries hispid.

HAB. Mountains. May. Ђ. Shrub small; leav. 5-lob.; raceme erect, 8-10-fl., gland.; ber. red.

* Stems thorny.

G. R. hirtellum: spines subaxillary; branches somewhat hispid; leaves small, half 3-cleft; lobes subdentate; peduncles 1 -flowered; berries smooth.

HAB. Mountains. May-June. h. Berries red.

7. R. gracile: spines subaxillary; leaves on slender petioles, pubescent on both sides; lobes acute. clentate, incised; peduncles slender, erect, about 2-flowered; calyx tubular-campanulate; berries smooth.

HAB. Mountains. May. Ђ. Spines mostly solitary; pet. short, white; ber. green or purp., sometimes with several spines.

8. R. triflorum: spines subaxillary; leazes smooth. 3-5-lobed, incisely toothed; peduncles about 3flowered; pedicels elongated; bracts very short; petals spathulate, undulate; style hairy, exserted. deeply 2 -cleft; berries smooth. 
HAB. Mountains. May. . Branch. spreading; spines sometimes 0 ; fl. purp.-green; ber. small, red.

*. R. oxycanthoides: larger spines subaxillary ; smaller ones scattered over the stem; leaves smooth; lobes dentate; peduncles short, about 2-flowered; berries smooth.

HAB. Rocks. h. Bran. slend.; leav. sub-3-lobed; fl. solit. or in pairs, pale yell.; ber. purp., glob.

10. R. lacustre: subaxillary spines compound; stem on every part hispid-aculeate; leaves lobed beyond the middle ; petioles villous ; berries racemose, hispid.

HAB. Mountain swamps. June. $\eta_{2}$. Stem reddish; leav. 5-lob.; racem. pend., 5-6-fl.; pet. white; ber. browon.

11. R. Cynosbati: subaxillary spines by pairs; leaves with short lobes, incisely toothed, softly pubescent ; racemes nodding, few-flowered; calyx erectcampanulate ; berries aculeate.

HAB. Mountains. Apr.-June. 万. Fl. green; ber. dark browen.

184. HAMILTONIA. Oil-nut. Santalacece.

H. oleifera.

HAB. Mountains, near rivulets. May-June. Shrul 4-6 ft. high, very pubes.; leav. altern., ovate, entire; $f$. greenish, racemose; nut oily.

185. THESIUM. Bastard toad-flax. Santalacece.

'T. umbellatum: stem erect; leaves oval-lanceolate; fascicles of flowers terminal, subcorymbed. HAB. Rocky hills and woods. July-Aug. 24. Stem $1 \mathrm{ft}$. high; leav. altern., entire; fl. white.

\section{ANYCHIA. Illecebreo.}

1. dichotoma: stem erect or spreading, dichotomously branched; leaves lanceolate, smooth, acute ; flowers about as long as the stipules.

HAB. Dry woods and hills. June-Aug. $\odot$. Stem filif., pubes. above; leav. oppos.; f!. solit., very minute.

3. capillacea: very smooth; flowers spreading, longer than the stipules at their base. 
HAB. Pine-barrens. Aug. A span high; leaz. smaller, often obtuse.

GLAUX. Black Salt-wort. Primulacec.

G. maritima.

HAB. Sea-coast. 4 Erect, 4-5 in. high; lear. oppos., ovate, fleshy; $f$. small, solit., axill., rose col.

\section{DIGYNIA.}

188. APOCYNUM. Dog's-bane. Apocinece.

1. A. androsaemifolium: leaves ovate, smooth on both sides; cymes lateral and terminal, smooth; tube of the corolla longer than the calyx.

HAB. Fields and hedges. June-July. 4. Stem. 2-3 ft. high; branch. sprecte.; fl. pink; border spreading.

3. A. pubescens: stem erect; leaves ovate, hoary-pubescent beneath; cymes pubescent ; corolla longer than the calyx ; border erect.

HAB. Borders of fields. June-July. 4. Branch. few, erect; petiol. short.; $f$. few, sm., greenish.

3. A. hypericifolium: leaves oblong, smooth, on very short petioles, mucrnnate, obtuse and subcordate at the base; cymes shorter than the leaves; calyx nearly as long as the tube of the corolli.

HAB. Fields and borders of woorls. June-July. 4. Leav. subsess., very acute; cor. sinall, greenishwhite, erect.

4. A. cannabinum: leaves lanceolate, acute at each end, smooth on both sides; cymes paniculate; "calyx as long as the tube of the corolla.

HAB. Fields and borders of woods. June. 4 . Branch. slend.; cym. many-fl.; cor. sinall, campan.. greenish.

189. PERIPLOCA. Ipocinec.

P. groca: flowers hairy within, terminal. HAB. New-York. Aug. h. Climbing; leav 
oppos., avate, acuminate; fl. corymb. dark purp.; seg. linear. §.?

190. GONOLOBUS. Apocinece.

1. G. obliquus : stem climbing, hairy ; leaves ovatecordate ; villous, acute ; corymbs axillary ; segments of the corolla orate, acuminate, oblique, revolute.

IIAB. Moist rocks. July. 4. Leav. veined; corymbs short; $f$. foctid, dark-purp.

2. G. hirsutus: sarments and petioles very hairy ; leaves cordate-oval, distinctly acuminate, pubescent on both sides; segments of the corvlla oblong, obtuse ; follicles oblong, muricate.

$1 \mathrm{AB}$. Hedges, near rivulets. 4. Trailing or climbing; umb. 4-6-fl.; pet. dark-purp.; fol, with soft spines.

191. ASCLEPIAS. Silk-weed. Asclepiadea.

$$
\text { * Leaves opposite. }
$$

1. A. syriaca: stem subsimple; leares lanceolateoblong, petiolate, tomentose beneath ; urnbels nodding; nectary bidentate; follicles muricate.

IMB. Sandy fields, \&c. July-Aug. 24. Stem 2-4 ft. high; umb. 2-3, 15-20-fl.; fl. large, pale-purp.

2. A. phytolaccoides : stem erect, simple ; leaves broadlanceolate, acuminate, smooth, pale beneath ; umbels inany-flowered, lateral and terminal, solitary, on long peduncles, nodding; nectary bidentate.

HAB. Shady wet places. June-July. 4. Stem 3-4 ft. high; leav. large; umb. 6-10-fl.; $f$. greenish-purp.

3. $\Lambda$. variegata: stem simple, erect; leaves ovate, petiolate, smooth ; umbels lateral and terminal, on short peduncles, crowded; perlicels tomentose; horn broad-falcate.

HAB. Woods. July. 24. Stem 3-4 ft. high. pubes. above; umb. mostly 2, 20-30-fl. zwhite, rarely purplish.

p. A. obtusifolia: leaves amplexicaul, oblong, obtuse, undulate, very smooth, glaucous beneath; umbel.

b. 3 
terminal, long-pedunculate, gererally solitary , nectary slightly 2-toothed; horns exserted. HAB. Sandy fields and hills. June-July. I Stem 2-3 ft.high; leav. remarkably undulate; $f$. palepurp.

5. A. laurifolia: stem erect, smooth ; leaves subsessile, oval-lanceolate, tapering to the summit, very acute, smooth ; umbels on long peduncles, terminal and axillary.

HAB. Pennsylvania. Aug. 4. Stem 2 ft. high ; sunmit pubes.; leav. obtuse at base; $f$. green and purp.

3. A. incarnata : stem erect, branched abore, tomentose, leaves sub:essile, lanceolate, tomentose, umbels erect, generally by pairs; nectary entire; horns subulate, exserted.

HAB, Wet places. 4. Stem 2-3 ft. high; unl. num., crowd.; fl. pale-purp.; nect. truncate.

3. pulchra: stem and leaves very hairy.

i. A. amona: siem with 2 longitudinal pubescent lines; leaves subsessile, oblong-oval, pubescent beneath ; umbels terminal, erect; nectary entire; horn subulate, exserted.

HAB. Swamps and wet cueadows. July-Aug. 4 . Stem $3 \mathrm{ft}$. high, nearly smooth; umb. many-fl.,purp.

3. A. purpurascens : stem simple; lesves ovate, villous beneath; umbels erect; leaflets of the nectary resupinate.

HAB. Wet places. 24. Stem $1_{2}^{\frac{1}{f}}$ ft. hight, hairy above; leav. on short pet. : fl. purp.

1). A. debilis: very smooth; stem erect, weak, simple; leaves petiolate, oval-lanceolate, acute at each extremity, membranaceous ; umbels terminal, loose.

HAB. Shady rocky places. 4. Leaies large: flowers white.

10. A. acnminuta: stem erect, smooth, simple; leaves ovate, subcordate, acuminate, subsessile; umbels lateral, solitary, erect; nectary acute; horn. scarcely exserted.

HAB. Sandy swamps. Aug. 24. Root large, tub.; stem $18 \mathrm{in}$. high; umb. 2; fl. purp. and green. 
11. A. quadrifolia: stem erect, sirnple, smooth ; leaves by foura, ovate, acuminate, petiolate; umbels 2 , terminal, erect, loose; pedicels capillary; nectary bidentete'; horn very short.

HAB. Dry stony woods. June. 4. Stem $18 \mathrm{in.}$ high; leaves mostiy 8 ; 2 mildle pairs approx.: umnb. on 'nng pedunc.; fl. small, white.

12. A. viridiflora: stem erect, simple, huiry; leaves oblong, on short petioles, tomentose-pubescent on both sides, obtuse ; umbels lateral, solitary, subsessile, nodding, dense; horns of the nectar? wanting.

HAB. Sandy fields. July. 24. Stem $2 f t$. high, densely pubes.; leav. thick; unb. subglob.; $f$. green.

ß. lanceoluta: leaves lanceolate, acute.

$\gamma$. oborata: leaves obovate.

13. A. verticillata: stem simple, marked with pubescent lines; leaves mostly verticillate, narrowlinear, revolute; nectaries short, bidentate; horns falcate, much exserted.

HAB. Dry hills. July. \&. Stern about $3 \mathrm{ft}$. high, very slend.; whorles 5-6.leav. ; Al. sinall, rehitish.

* Isaflets alternate.

11. A. tuberosa: stem erect, hairy, with spreading branches; leaves oblong-lanceolate, scssile ; umbels numerous, forming terminal corymbs.

HAB. Simdy fielids. Aug. 4. Root, large, tub.: stem $3 \mathrm{ft}$. high, ; $f$. large, bright orange.

192. GENTIAN.S. Gentian. Gentiunc.

1. G. Suponariu: leaves ovate-lanceolnte, acute, 3 nerved; flowers verticillate-capitate, sessile; corolla ventricose, closed, 10 -cleft ; interior segr ments unequally 3-cleft, as long as the exterior ones; segments of the calyx ovate, shorter than the tube.

HAB. Wet mandows. Sept.-Oct. 4. Stem 18 in. high, simp.; leav. oppos. ; fl. very large, brighe blue. 
3. G. ochroleuca: stem subangular; leaves ovate. lanceolate; flowers in terminal subsessile fascicles; segments of the calyx linear-lanceolate; corolla campanulate-ventricose, 10-cleft; segments acute, slightly connivent; interior ones sub. dentate, short.

HAB. Fields and woods. Sept. 4. Stem 1 ft. high, subsulc.; fl. large, yellowish-white.

3. G. Pneumonanthe: stem terete; leaves linear-lanceolate, obtuse ; flowers few, terminal, (and axillary,) subsessile; segments of the calyx linear-oblong. rather acute ; corolla campanulate, 5 -cleft ; segments acute ; interior plaits short, 1 -toothed.

$\mathrm{HAB}$. High mountains. 4. Stem a foot high, very smooth; fl. large, bright blue.

4. G. angustifolia: stem simple, slencler, 1-flowered; leaves linear, spreading; corolla infundibuliform, 5 -cleft, with 5 interior lacerate segments.

HAB. Sandy fields. Oct.-Nov. 4. Stem $1 \mathrm{ft}$. high, slend.; leav. long; l. 2 in. long, azure-blue.

.. G. linearis: stem somewhat scabrous; leaves linearlanceolate, undulate, ciliate, as are also the segments of the calyx; flowers sessile, in terminal crowded fascicles; corolla campanulate, 5 -cleft ; segments obtuse, with the interior folds denticulate.

HAB. Mountains. 4. Fl. middle-sized, blue; seg. of the cor. very short.

d. G. quinqueflora: stem quadrangular, branched; leaves ovate-lanceolate, acute, 3-nerved; flowerf. somewhat in tives, terminal and axillary, pedicellate; corolla tubular-campanulate, 5 -cleft ; segments setaceously acuminate; calyx very short.

IIAB. Woods and hill sides. Sept.-Oct. \&. Stem 1 ft. high, rarely simp. ; fl. small, pale blue.

$\because$ Cr. crinita: stem terete ; branches elongated, 1 Howered; leaves lanceolate, acute; corolla half 4-cleft; segments incisely ciliate.

IIAB. Wet meadows. Oct.-Nov. §. Stern $18 \mathrm{in.}$ high, quadrang. above; fl. very large, blue.

193. CUSCUTA. Dodder. Convolvuli.

1. C. americana : flowers pedunculate, umbellate, cleft ; stigmas capitate ; corolla tubular-6ampanalate. with the border small and spreading. 
HAB. Low grounds. June-Aug. $\odot$. Stems filif., orange, parasit., treining; $f$. in dense clust.

2. C. europcea: flowers sessile; corolla 4-5-cleft, without scales it the base of the stamens ; stigmas simple.

HAB. Parasitic on flax. July. $\odot$. Stems filif., red, twining; fl. yell., campan.

\section{HEUCHERA. Alum. Saxifragce.}

1. H. americana : viscidly-pubescent; scape and leaves a little scabrous; leaves with rounded lobes, dentate; teeth dilated, obtuse, mucronate; panicle dichotonous; calyx short, obtuse ; petals lanceolate, as long as the callyx.

HAB. Shady rocky places. June-July 4. Leav. rad. on long pet.; scupe $2-3$ ft. long.; fl. purp.

$\therefore$ H. pubescen : pulverulent-pube:cent; scape smooth below; leaves snmewhat acutely lobed, smooth beneath, toother ; teeth rounded, mucronate; peduncles short, with crowded flowers; calyx large, campaulate; petals !onger than the calyx; sta" mens scaicely exserted.

HAR. Mouritains. 4. Leav. with broad teeth; scape $2 f t$. long, scab above; pet. red und yell.

195. PANAX. Ginseng. Iralice.

1. P. trifulium: lraves teruate; leatlets subsessile; styles 3 ; berry tricurcous; rnot globose.

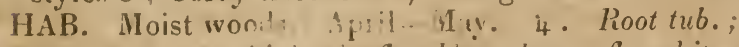
stem 4-8 2 .high; leafl. oblong-lan.; $f$. wohite; ler. grten.

2. P. quinq"efolium: leaves in threes; leaflets quinate. pitiolite; peduncles shorter than the petioles; root fusifnrm.

HАB. Winustains. July. H. Root long, wrinkled; stem $1 \mathrm{ft}$. high; $f$. greenish; ber. renif., scarlet.

196. ATRIPLEX. Orache. Chenopodec.

1. A. hortensis : stem erect, herbaceous ; leaves triangular, dentate, green on both sides; fructiferous ralyx ova'e, reticulate, entire.

HAB. Waste places, Sc. July-Aug. ๑. Stem 3-1 ft. high, green; fl. green, in interrup. spikes. 
2. A. arenaria: stem herbaceous, spreading; leave oblong-ovate, subsessile, silvery-mealy veneath, very entire ; upper ones acute or acuminate ; fructiferous caly $x$ muricate, dentate, retuse.

HAB. Sea-coast. Aug.-Sept. ๑. A foot high, much branch.; leav. 1 in. long; cal. 3-lnbed, large.

3. A. laciniuta $\beta$. omericuna: stem herbaceous, diffuse; leave triangular hastate, deeply toothed, glaucous, a little pulverulent; perfect flowers tetandrous; fructiferous calyx rhomboid, acute, entire.

HAB. Salt marshes. Aug. ๑. Erect or procumb.; leav. petiol, gray; fl. in glom. racemes.

1. A. patula: stem herbaceous, spreading; leaves triangular-hastate, smóth above, irregular!y toothed; the upper ones entire; fructiferous calyx submuricate on the sides.

HAB. New-York. +

3. A. Halimus: stem frutescent; leuves alternate and opposite, subrhomboid, very entire.

HAB New-Jersey.+

197. CHENOPOUIUM. Goose-foot. Chenopodea.

1. C. Bonus Henricus: leaves triangular-hastate, very entire; spikes compound, terminal and axillary, erect, leafless.

HAB New-York, \&c. June. «. Stem. 1 ft.high; leav. large, dark green; fl. green, polyg.

2. C. rhombifolium: leaves triangular-rhnmbic, acute, repandly toothed; racemes axillary, erect, leafless; bracts minute, inflexed.

HAB. Pennsylvania. June. $\odot$. Leav. acutely tooth.; racem. simp., short.

3. C. album: leaves rhomboid-ovate, erose, entire at the base; upper ones oblong, very entire ; racemes branched. somewhat leafy; seed smooth.

HAB. Cultivated grounds, \&c. July-Sept. ○ . Stem erect, 2-4 ft. high; leaves mealy.

$\beta$ viride: leaves greener and more entire; racemes more branched, a little leafy.

b. C. hybridum: leaves ovate-cordate, acuminate, angularly toother ; racemes axillary, paniculate, divaricate, leafless.

HAB. Waste places, \&c. July-Aug. ○. Stem. 2$3 \mathrm{ft}$. high ; leav. large, nearly smooth, bright green: 
э. C. rubrum: leaves rhomboid-triangular, deeply toothed and sinuate; racemes erect, compound, leafy.

HAB. Waste places. July. ๑. Stem. reddish; leav. dark green; glomerules minute.

6. C. ambrosoides: leaves lanceolate, remotely toothed ; racemes simple, axillary, leafy.

HAB. Waste places. Aug.-Oct. ๑. Much bran., often diffuse, strong-scented; leav. small.

7. C. Botrys: leaves oblong, sinuate; racemes naked, much divided.

$H A B$. Sandy waste places. Aug.-Sept. $\odot$. One ft. high, sweet-scented, riscid-pubes; $f$. distinct.

8. C. anthelminticum: perennial ; leaves oblong-lanceolate, toothed; spikes long, interrupted, leafless ; style 3-cleft.

HAB. Sandy fields. Sept. $\odot$ ? Strong-scented, $1 \frac{1}{2}-\mathrm{e}$ ft. high, often reddish; racem, axill. and term.

9. C. glaucuin: leaves oblong, repand, glaucous beneath ; spikes simple, naked, glomerate, axillary and terminal.

HAB. New-York. ๑. Stem diffuse, thick; racemes short, lobed, leafless.

10. C. maritimum: leaves linear, fleshy, semicylindrical ; flowers axillary, glomerate.

HAB. Salt marshes. Aug.-Sept. $\odot$. Stem $1 \frac{1}{2}-2 f t$. high; glomerules small; seed spiral.

198. SALSOLA. Salt-wort. Chonopodere.

1. S. Kali: herbaceous, decumbent; leaves subulitt; canaliculate, spinose ; flowers axillary, solitary ; fructiferous calyx with a scarious margin.

HAB. Sea-coast. Aug. ๑. Stem much bran., pules. ; cal. spreading; leav. subulate.

3. caroliniana: leares dilated; calyx with a broader margin.

HAB. Sandy fields and waste places, near salt water. Cal. depress., reddish.

2. S. Tragus: herbaceous, spreading, smooth; leares subulate, fleshy, mucronate-spinous; flowers subsolitary; calyx subovate; margin flattened, discolnured.

HАB, New.Jersey, \&c. July. + . 
3. S. Soda: herbaceous, smooth; branches ascending; leaves semiterete, rather acute; fructiferous calyx transversely carinate in the middle, somewhat membranaceous.

HAB. New-York. +

\section{ULMUS. Elm. Ulmacece.}

๖. U. americana: branches smooth; leaves somewhat doubly serrate, unequal at the base ; serratures uncinately acuminate; flowers pedicellate; fruit fimbriate.

HAB. Woods. April. A large tree; bran. long, recurv.; leav. altern.; $f$. purplish, small appearing before the leav.

$\therefore$ U. fulva: leaves oval-oblong, much acuminate, pubescent on both sides; buds tomentose; flowers sessile.

HAB. Rocky hills. April. Tree 20-25 ft. high; fl. conglom., ciliate; stam. 7.

3. U. nemoralis: leaves oblong, somewhat smooth, equally serrate, nearly equal at the base; flower: sessile.

HAB. River banks. +.

200. CELTIS. Nettle-tree. Ulmacer.

1. C. occidentalis : leares orate, acuminate, equally serrate, unequal at the base, scabrous above, pubescent beneath ; flowers subsolitary.

HAB. Wouds. May. Tree middle-sized; leav. altern.; fl. small, greenish-zellite; drupe purp.

2. C. crassifolia: leaves ovate, acuminate, unequally serrate, subcoriaceous, scabrous on both sides, unequal at the base; peduncles mostly 2 -flowered.

HAB. River banks. Tree smaller than . No. 1 .

3. C. pumiia: leaves ovate, acuminate, equally serrate, unequal at the base; the younger ones only pubescent; peduncles mostly 3 -Howered ; fruit solitary. HAB. Banks of rivers.

201. ERYNGIUM. Eryngo. U'mbelliferce.

1. E. aquaticum: leaves linear-lanceolate, remotely ciliate-spinous; inferior ones ensiform, those of the flowers lanceolate, dentate ; involucrum shorter than the globose capitulum, entire, as are also the scales of the receptacle; stem subdichotomous. 
HAB. New-Jersey? 4. Root tub.; stem 2-3 fi. high, trichot. above; leav long; fl. woh. or pale blue.

2. E. virginianun: leaves long-lanceolate, serrate; involucrum longer than the heads; chaff tricuspidate. HAB. Shores of rivers. 4. Root tub.; stem $3-4 \mathrm{ft}$. high; leav. 6 in. lnng; heads num.; fl. woh. or biue.

3. E. virgatum: leaves spathulate-ovate, irregularly toothed; leaflets of the involucrum linear, longer than the glohose heads; chaff tricuspidate.

HAB. Wet meadows. 4 ? Stem 2-3 ft. high; leav. 2 in. long, acutely dent.; heads small; fl. blue.

202. SANICULA. Sanicle. Umbelliferce.

S. murilandica: leaves all digitate; leaflets oblong. incisely serrate; staminiferous flowers numerous, pedicellate.

HAB. Woods and thickets. June-Aug. 4. Stem 2 ft. high; $f$. in small capit., umb. white.

3. canadensis: leaves subternate; leaflets ovate. coarsely toothed.

203. HYDROCOTYLE. Marsh penny-wort。 Umbelliferce.

1. H. vulgaris: leaves peltate, orbicular, crenate; umbels capitate, about 5 -flowered.

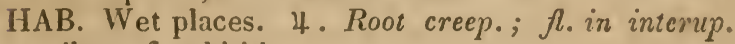
spikes; fl. wohitish.

2. H. umbellata: leaves peltate, crennte, emarginate at the base; umbel of many pedunculate flowers.

HAB. Wet places. 4. Root creep.; leav. subrenif.; umb. 20-30-fl., longer than the leav.; fl. renif.

3. H. americana: smooth; root tuberous; leaves reniform, somewhat 7-lobed, crenate; umbels fewflowered, sessile.

HAB. Wet shady places. June-Aug. 44. Crecping; stem filif.; umb. very small, glomerate.

4. H. ranunculoides: leaves somewhat 5-lobed, cordate, crenate-dentate; umbels capitate, 8-10-Howered. IIAB. Pennsylvania. June. 4. Leav. nearly circular; pedunc. 1 in. long; pediccls very short.

H. lincatu: very smooth, creeping; leaves scssile: cuneate-linear, with transverse nerves; umbel pedunculate. 
HAB. Muddy banks. July-Aug. 4. Leav. $1 \frac{1}{2}$ inv. long, succul., with 5 transv. nerves; umb. 8-10-fl.

6. H ? composita: root tuberous; stem simple, 2-leaved; leaves subternate; leaflets many-cleft; umbel $3-5$ flowered.

HAB. Alluvial soils. April. 4. Roat round; stem ascend., 1-leaved; scapes $4-5 \mathrm{in}$. high; fl. wohite.

204. SISON. - Honey-wort. Umbelliferce.

1. S. aureus: stem nearly simple, sulcate; leaves biternate, shining; leaflets oblong-lanceolate, attenuate at the base, incisely serrate; involucrum 0.

HAB. Rocky hills. June-July. 24. Stem $1 \frac{1}{2}-2$ ft。 high ; umb. comp.; fl. yell.; fruit dark-col.

g. S. integerrimus: stem nearly simple; leaves bi-triternate, glaucous, very entire; leaflets oval, entire; umbel with elongated rays; involucrum 0.

HAB. Meadows and mountains. June. 4. Siem 18 in. high; rays of the umb. filif.; fl.yell.; fruit black.

3. S. capillaceus: leaves decompound, filiform; stem dichotomous, angular; involucrum subpinnatifid.

HAB. Brackish meadows. July-Oct. ○. Much bran.; 1 ft.long; umb. axill., pedunc.; fl. white.

205. CNIDIUM. Umbelliferce.

1. C. canadense: stem angular, flexuons; leaves bipinnate, shining; leaflets many-parted; segments lanceolate.

HAB. Mouths of rivers. +

2. C. atropurpureum: radical leaves subcordate, simple, serrate; cauline ones ternate; leaflets ovate, acute, subcordate ; middle one petiolate; partial involucra dimidiate, 3-leaved.

HAB. Rocky banks of rivers. June. $2 \%$. Stem $2-3$ fi. high; fl. dark-purple.

206. SMYRNIUM. Alexanders. Uinuelliferce.

S. cordatum : radical leaves simple, cordate, crenate; stem-leaves ternate, serrate; umbels terminal.

HAB. Rocky hills. May-June. 24. Stem 2-8 ft. high; rad. leav. on lons pet.; fl. yell.; fruit black. 


\section{CICUTA. Umbellifera.}

1. C. maculala: stem spotted; leaves triternate; leaflets subternate, lanceolate, acuminate, mucronately-serrate; umbels axillary and terminal ; partial involucra setaceous.

HAB. Wet places. July-Aug. 7. Stem 3-6 ft. high, glauc.; umb. large, slend.; fl. white. Poisonous!

2. C. bulbifera: leaves various, ternate and biternate, bulbiferous; leaflets linear and linear-lanceolate, remotely toothed; umbels terminal, solitary.

HAB. Swamps. Ang. 4. Stem $2-3 \mathrm{ft}$. high, slend.; leav. bulbif. in the axils.; umb. small; fl. wh.

208. DAUCUS. Carrot. Umbellifera.

D. Carota : stem hispid; leaves tripinnate; leaflets incised, linear-lanceolate, acute; umbel at length concave ; fruit bristly.

HAB. Old fields. June-Aug. ơ. Stem $2 \mathrm{ft}$. high; leav. pale green; fl. white.

209. MYRRHIS. Umbelliferce.

1. M. procumbens : stem procumbent, somewhat hairy ; leaves bipinnate; segments lanceolate, rather obtuse ; umbels 3-rayed, few-flowered ; fruit smooth. HAB. Shady rocky pláces. May. ๑. Stem 6-8 in. long; fl. all fert., white; fruit hinear.

2. M. Claytoni: stem hairy, (at first hoary-white ;) leaves biternate, pubescent; leaflets incisely lobed, dentate ; umbel 3-rayed ; central flowers abortive ; universal and partial involucra 3-5-leaved, lan. cenlate, ciliate; fruit attenuate at the base, with hispid angles, not rostrate; style very short.

HAB. Shady rocky places. May-June. 4. Root fusif.; stem 2 ft. high; fruit linear-lan., blackish.

3. M. longistylis : stem smooth; leaves biternate; the lower ones on short petioles; leaflets ovate, incisely lobed and dentate; umbel 3-4-rayed; central flowers abortive ; universal and partial involucra 3-5-leaved, oblong, acuminate; fruit attenuate at the base, with hispid angles; styles long, subulate, straight. 
HAB. Wet meadows. June. 4. Stem 2-3 $f i$. high; leav. slightly hairy; purtial petiol. elong. ; fl. $w$.

1. M. canadensis: leaves ternate, smooth; leaflete rhomboid-ovate, acute, incisely toothed, acutely serrate; partial involucra minute, subulate; fruit oblong, very smooth.

HAB. Rocky woods. June. Li. Stem $1 \frac{1}{2}-2 f t$. high, erect, smooth; umb. num.; fr. 3 lin. long.

210. SIUM. Water-parsnip. Umbelliferce.

1. S. latifolium: stem angular; submerged leaves bipinvatifid; upper ones pinnate; leaflets oblonglanceolate, unequal at the base, acutely serrate; umbels terminal.

HAB. Shady swamps. July. 4. Root creep.; stem 17-ang., 18 in. high; leaft. 4 pairs; fl. zhite.

3. S. lineare: stem angular and sulcate; leaves pinnate ; leaflets $4-5$ pars, linear-lancer 1 tr arutely and finely serrate; involucra many-leaved, linear : umbels terminal.

HAB. Swamps. Aug. 4. Stem 7-ang, 2-3 ft. high; leaft. long and narrow; fl. white; cal. obsol.

\section{CONIUM. Hemlock Umbellifera.}

C. maculatum: stem very smooth, spotted; leaves tripinnate; leaflets lanceolate, pinnatifid ; segments lanceolate, nearly entire.

HAB. Road sides, \&c. July. o. Stem 2-4 ft. high; leav. smooth and shin.; fl. zehite. Poisonous !

212. LIGUSTICUM. Lovage. Umbelliferce.

l. L. scoticum: leaves biternate ; leaflets subrhombicovate, coarsely serrate ; involucrum linear-lanceolate.

HAB. Salt marshes. July. 4. Stem $18 \mathrm{in.} \mathrm{high,}$ smooth; leav. retic.; uinb. pedunc.; $f$. wuh.

2. L. actceifolium: leaves biternate; leaflets oval, equally toothed; partial involucra setaceous ; fruit oblong-oval, leaves somewhat wings.

HAB. New-York. 4. Siem very tall, smooth; petioles long; umb. num., subvert. ; fl. woh. 


\section{HERACLEUM: Cow-parsnip. Umbellifere.}

H. lanatum: leaves ternate, petiolate, tomentose beneath ; leaflets petioled, round-cordate, lobed;

- fruit orbicular.

HAB. Wet meadows. June. 4. Stem 3-5 ft. high, thick, pubes.; umb. large; $f$. wh.

\section{PASTINACA. Parsnip. Uimbelliferce.}

1. P. sativa: stem sulcate; leaves pinnate; leaflets subpubescent beneath, oblong, incised; terminal one 3-lobed.

HAB. Fields, \&c. July-Sept. 8. Stem 2 ft. high, smooth; leafl sess.; fl. yell.

2. P. rigida: stem terete striate; leaves pinnate, smooth; leaflets oblong-lanceolate, entire, or with several remote teeth.

HAB. Sivamps. Sept. 4. Stem 2-4 ft. high, rigid; leafl. $4-5$ pairs; fl. white.

3. P. ambigua: stem slightly angular; leaves pinnate, smooth; leaflets linear, entire, subglaucous beneath ; umbels terminal, subsolitary ; partial involucra subulate.

HAB. Swamps. Sept. 4. 3-5 ft. high, slend.; fl. wh.

\section{ANGELICA. Umbelliferce.}

1. A. triquinata: stem terete, pubescent above; leaves ternate, very smooth; the partitions quinate; leaflets oblong-ovate, equally serrate; the inferior ones 2 -lobed at the base.

HAB. Dry woods. Aug. 4. Stem 3-5 high, straight, white above, leav. thick; $f l$. white.

2. A. atropurpurea: stem smooth, coloured; leaves ternate; the partitions subquinate; leaflets ovate, acute, incisely serrate, sublobed; the 3 terminal ones confluent ; petioles very large, inflated.

HAB. Wet meadows. June. 4. Root aromat.; stern 3-5 ft. high, thich, purplish ; fl. greenish.

3. A. lucida: leaflets equal, ovate, incisely serrate. $H \Lambda B$. Shady woods. 4. Stem 1-2 high; rad? leav. tripin.; leafl. dark green, lucid above.

M 2 


\section{THAPSIA. Uinbelliferce.}

T. trifoliata: petioles and nodes of the stem pubescent; leaves biternate and ternate; leaflets cuneate and ovate, unequally and incisely serrate, entire at the base; umbels terminal ; partial involucra 3-leaved, subulate.

HAB. Sandy banks of rivers. June. 4. Stem 3 ft. high, ang. and sulc.; umb. 3-4; fl. yell.

\section{TRIGYNIA.}

\section{VIBURNUM. Caprifolia.}

1.V. prunifolium: smooth; leaves round-obovate, acutely serrate; petioles margined.

HAB. Woods and hedges. June. A small tree, leav. oppos., $1 \frac{\mathrm{t}}{2} \mathrm{in}$. long ; fl. white; ber. oval, dark blue.

$\therefore$ V. pyrifolium: smooth; leaves ovate-oblong, acute, crenate-serrate ; petiole naked; cymes subpedunculate; fruit ovate-oblong.

HAB. Rocky woods. June. Shrub 5-10 ft. high; leav. 2 in. long; bei. red.

3. V. Lentago: smooth; leaves broad-ovate, acuminate, acutely serrate; petioles margined, undulate; cymes sessile.

HAB. Rocky woods. May. Shrub 8-12 ft. high: leav. 3 in. long, often subcord.; ber. black.

1.V. nudum: leaves oval-oblong; margin revolute and obscurely crenulate; petioles naked; cymes pedunculate.

HAB. Swamps. June. Shrub 3-10 fi. high; leav. 4 in. long, punct. and retic. beneath; ber. bluc.

-. V. Lantanoides: leaves orbicular-cordate, abruptly acuminate; nerves and petioles pulverulent-tomentose, unequally serrate; cymes closely sessile.

HAB. Rocky woods. May-June. Slirub $4-8 \mathrm{fl}$. high; leav. large, with the nerv. rusity; cym. often radiate.

ฬ. V. dentatum : nearly smooth; leaves on long petioles, orbicular-ovate, dentate-serrate, plicate; axils of the veins pubescent beneath ; cymes pedunculate : fruit subglobose. 
HAB. Woods and hedges. June. Shrub $8 \mathrm{ft}$. high, with straight bran.; ber. small, blue.

7. V. pubescens : leaves on wery short petioles, ovate, acuminate, dentate-serrate, villous beneath: cymes pedunculate; fruit oblong.

HAB. Mountains. June. Shrub $6 \mathrm{ft}$. high; leav. and cymes swaller than No. 6.

3. V. acerifolium: leaves subcordate, 3-lobed, acutely serrate, pubescent beneath; lobes acuminate, petioles without glands, hairy; cymes on long peduncles,

HAB. Rocky woods. May-June. Shrub 4-6 ft. high; leav. broad; ber. oval, compress., blackish.

9. V. Oxycoccus: leares S-lobed, acute at the base, 3-nerved ; lobes divaricate, acuminate, remotely and obtusely dentate; petioles glandular; cymes radiate.

HAB. Mountain woods. May-June. A small shrub; branch. spread.; ber. large, red, acid.

10. V. edule: leaves 3-lobed, rather obtuse at the base, 3 -nerved; lobes very short, with acuminate-dentate serratures; petioles glandular; cymes radiate.

HAB. Banks of rivers. Shrub smaller and more upright than the last; ber. large, red, acid.

\section{SAMBUCUS. EIder. Caprifolia.}

1. S. canadensis: nerves and petioles very smooth; leaflets oblong-oval, about 3 piirs, acuminate. smooth ; midrib subpubescent ; cymes lax ; stem frutescent.

HAB. Low grounds. May-July. Shrub 6-10 ft. high ; leuv. often bipin.; $f$. zchite, ber. deep purp.

$\therefore$ S. pubens: petioles and leares beneath pubescent, leaflets oval-lanceolate; cymes paniculate; stem fruticose.

IIAB. Mountains. June. Shrub 6-3 ft. high : lear. simply pinnate, 3 pairs; ber. small, red. 
219. RHUS. Sumach. Terebintacer.

* Leaves pinnate.

1. R. glabrum: leaflets lanceolate, smooth, acuminate, acutely serrate, whitish beneath; flowers perfect ; fruit downy.

HAB. Hedges and thickets, July. Shrub 6-12 ft. high, with strag. branch.; leafl. 12-15 pairs; ber. crim.

2. R. typhinum: branches and petioles very villous; leaflets in many pairs, lanceolate-oblong, acuminate, acutely serrate, pubescent beneath.

HAB. Rocky hills. June. Shrub 8-15 high; leafl. 10-15 pairs; pan. dense, oblong; ber. purp. vill.

3. R. copallinum: petiole winged; leaflets in many pairs, oval-lanceolate, very entire, shining on the upper surface; panicle sessile; flowers dioecious. HAB. Dry woods and hill sides. July. Shrub 4-6 ft. high; leafl. dark-green, 4-6 pairs; ber. red, hairy.

4. R. Vernix: very smooth; leaflets in many pairs, oval, abruptly acuminate, entire; panicle loose, flowers dioiecous.

HAB. Swamps. June-July. A small tree; leaft. 5 puirs, subsess.; pan. clust.; ber. smooth, zehitish.

* Leaves ternate.

b. R. Toxicodendron: stem erect; leaflets broad-oval, entire or sinuate-dentate, subpubescent beneath ; flowers dioecıous, in sessile axillary racemes.

HAB. Dry woods. June-July. Shrub 1-3 ft. high, smooth; leav. shin. above; ber. smooth.

$\beta$. radicans : stem climbing.

HAB. Woods and hedges. Stem climbing very high.

6. R. aromaticum: leaflets sessile, ovate-rhomboid, dentate, pubescent beneath ; flowers amentaceous, dioecious; berries hairy.

HAB. Mountains. April. Shrub small; $f l$, in axill. racem. or aments, ; ber. clust., red. 
220. STAPHYLEA. Bladder-nut. Celastrince.

S. trifolia: leaves ternate.

HAB. Rocky hills. May. Shrub 6-10 ft. high; fl. in pend. racem., greenish-zoh.; caps. large.

\section{TETRAGYNIA.}

\section{PARNASSIA. Grass of Parnassus.} Uncertain.

1. P. caroliniana: radical leaves orbiculitr-ovate, cordate; nectaries 3-bristled.

HAB. Boggy woods. Aug.-Sept. 4. Leav. mostly rad., on long pet.; fl. sol., term., yellowish-wh. 2. P. palustris: radical leaves cordate; nectaries: many bristled.

HAB. Bog-meadows. +.

\section{PENTAGYNIA.}

\section{ARALIA. Aralice.}

1. A. nudicaulis: nearly stemless; leaf solitary, triqui. nate; scape naked, shorter than the leaf; umbels few.

HAB. Among rocks. June-July. Root thict. creep.; petiole long; leafl. oval, serrate; scape 1 fl., long, 3-umbelled ; fl. greenish.

2. A. racemosa: stem herbaceous, branclied; petioles 3-parted; partitions ternate and quinate; umbels compound, in axillary panicles.

HAB. Rocky woods. July-Aug. 4. Root thick, aromat. ; stem 3-4 ft. high; umb. num.

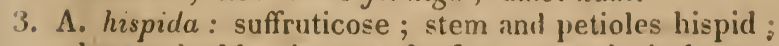
leaves doubly pinnate ; leaflets ovate, incisely serrate; umbels on long peduncles.

HAB. Mountains. July-Aug. Stem $1 \frac{\mathrm{I}}{2} \mathrm{ft}$. high, very hispid below; pedunc. axill. and term.

4. A. spinosa: arborescent; stem and leaves spinous; leaves doubly pinnate; umbels numerous, in compound panicles. 
HAB. Low fertile wonds. Shrub 8-12 jt. high, very prickly; pan. very large, term.

223. STATICE. Thrift. Plumbaginece.

1. S. Armeria: scape simple, terete, capitate ; leaves linear, flat.

HAB. Sea-shore. 4. Leav. all rad, cespit.; scape $1 \mathrm{ft}$. high; fl. rose-col.. in a term. head. +.

2. S. Limonium: scape paniculate, terete ; leaves oblong, undulate, smooth and nerveless, mucronate below the tip.

HAB. Salt marshes. Aug.-Oct. 4. Root large; scape $1 \mathrm{ft}$. high; pan. large; fl. secund, blue.

\section{LINUM. Flax. Lineo.}

1. L. virginianum: stem paniculate at the summit; radical leaves obovate or spathulate ; cauline ones lanceolate; flowers remote, alternate; segments of the calyx acute.

HAB. Rocky hills. July-Aug. ๑. Stem $1 \frac{1}{2}-3$ ft. high, slend.; leaves scat.; fl. very small, pale yell.

L. usitatissimum segments of the calyx ovate, acute, 3-nerved; petals crenate ; leaves lanceolate, alternate; stem subsolitary

HAB. Fields. June-Jaly. ๑. Stem 1-2 ft. high, bran. above; fl. large, blue.

\section{SIBBALDIA. Rosacece.}

S. procumbens: leaves ternate; leaflets cuneate, tridentate, smooth inove, hiry beneath ; flowers corymbed; petals lancenlate, acute, about as long as the calyx.

HAB. High mountains. If Plant small, procumb.; f. yellow.

\section{HEXAGYNIA.}

226. DROSERA. Sun-dew. Droseracece.

3. D. rotundifolia: leaves suborbicular, dilated; petiole elnngated, hairy above; racemes mostly simple, erect. 
HAB. Bogs. July-Aug. 4. Leav. all rad., spreading, covered with brown viscid filam.; scape 4-8 in. long; fl. secund, small, white; seeds lin.

2. D. longifolia: leaves crenate, obovate, tapering below into a long footstalk, ereci-spreading, scape declined at the base; stipules many-cleft, capillaceous; segments of the calyx ovate-oblong, obtuse. HAB. Bogs and sandy swamps. July-Aug. 4. Caudex sometimes elong.; scape bent and ascend.; seeds ovate.

3. D. filiformis : leaves filiform, very long, glandulous the whole length; scape simple or bifid.

HAB. Sandy swamps. Aug.-Sept. 4. Leav. 6-10 in. long; scape $1 \mathrm{ft}$. long: fl. large, purp.

\section{POLYANDRIA.}

227. XANTHORHIZA. Yellow-root. Ranunculacee.

X. apiifolia.

HAB. Banks of rivers. April. Suffruticose; roo large, yell.; stem 2-3 fl. high; lect. bipinn.; fl. in. comp. racem., dark-purp.

\section{HEXANDRIA.}

\section{MONOGYNIA.}

A. Flowers complete, having a calyx and corollü.

328. TRADESCANTIA. Cal. 3-leaved. Pet. is. Fil. villous. Caps. superior, 3-celled, manyseeded.

220. LEONTICE. Cal.6-leaved, caducous. Pet.6, unguiculate, opposite the calyx. $\mathcal{N}$ ect. 5 , inserted upon the claws of the petals. . Anth. adnate to the filam.; 2-celled; cells opening longitudinally. 
Per. rupturing at an early period, leaving the large naked, drupe-form seed elevated on its funic.

230. BERBERIS. Cal. 6-leaved. Pet. 6, with 2 glands on each claw. Style 0 ; stig. umbilicate. Berry 1-celled, 2-4-seeded.

231. PRINOS. Cal. minute, 6-cleft. Cor. monopetalous, subrotate, 6-parted. Berry 6-seeded. 32. FLOERKEA. Cal. 3-leaved. Cor. 3-petalled, shorter than the calyx. Style bifid. Utric. 2-3, united, 1-seeded, superior.

B. Flowers spathaceous.

333. AMARYLLIS. Cor. superior, 6-petalled, irregular. Fil. arising from the orifice of the tube, declined, or straight, unequal.

23\%. ALLIUM. Cor. 6-petalled, spreading. Spath many-flowered. Umb. crowded. Caps. superior, 3-celled, 3-valved, many-seeded.

235. HYPOXIS. Spath. 2-valved. Cor. superior, 6r parted, persistent. Caps. elongated, narrowed at the base, 3-celled, many-seeded. Seeds roundish, naked.

936. PONTEDERIA. Cor inferior, 6-cleft, bilabiate ; under side of the tube perforated with 3 longitudinal foramina; lower part persistent, calycine. Stam. unequally inserted; 3 of them upon the summit of the tube. Utric. muricate, 1-seeded.

C. Flowers naked. (Without a spath; perianth single, petaloid.)

237. CONOSTYLIS. Cor. semi-superior, 6-cleft, persistent, woody. Anth. erect. Style conic, tripartile; stig. simple. Caps. opening at the summit, 3-celled, 3-valved, many-seeded.

238. ALETRIS. Cor. tubulous-ovate, 6-cleft at the summit, rugose, persistent. Stam. inserted upon the orifice. Style triquetrous, tripartile. Caps. semisuperior, many-seeded, opening at the summit.

239. HEMEROCALLIS. Cor. campanulate; tube cylindric. Stum. declined. Stigmas rather small. simple, somerwhat villous. 
210. AGAVE. Cor. superior, erect, tubular or infundibuliform. Staminif. fil. Ionger than the corolla, erect. Caps. triangular, many-seeded.

241. PHALANGIUM. Cor. 6-petalled, spreading. Fil. smooth. Caps. superior, ovate. Seeds angular.

242. NARTHECIUM Cor. inferior, 6-petalled, spreading, persistent. Fil. filiform, hairy. Caps. prismatic, 3-celled, many-seeded. Seeds finely attenuate at each extremity. Cal. 0.

943. ORNITHOGALUM. Cor. 6-petalled, persistent, spreading above the middle. Cal. 0 . Fil. dilated at the base. Caps. superior, roundish, 3celled. Seeds roundish.

244. LILIUM. Cor. 6-petalled, campanulate. Pet. mostly reflexed, marked with a longitudinal nectariferous line. Stam. shorter than the style. Stig. entire. Caps. superior, subtriangular; valves connected by cancellate hairs.

45. ERYTHRONIUM. Cor. 6-petalled, subcampanulate ; petals reflexed; the interior ones with a callous tooth on each side near the base, and a nectariferous pore. Caps. superior, substipitate. Seeds ovate.

246. UVULARIA. Cor. inferior, 6-petalled, erect; petals with a nectariferous cavity at the base of each. Fil. very short; anth. adnate. Stig. reHlexed. Caps. triangular, 3-celled, 3-valved; valves septiferous in the middle. Seeds numerous, subglobose, axillate at the hilum.

247. STREPTOPUS. Cor. inferior, 6-petalled, subcampanulate. Stigmas very short. Berry subglobose, smooth, 3-celled. Seeds few, hilum naked.

948. CONVALLARIA. Cor. inferior, G-cleft. Berry globose, spotted, 3-celled.

249. ASPARAGUS. Cor. inferior, 6-parted. Style very short ; stignas 3. Berry 3-celled, cells 2seeded. 
D. Flowers incomplete. (Perianth single, resembling a calyx.)

250. ORONTIUM. Spad. cylindric, crowded with flowers. Cor. 6-petalled, naked. Style and stig. scarcely any. Utric. 1 -seeded.

251. ACORUS. Spad. cylindric, covered with flowers. Cor. 6-petalled, naked. Stig. sessile, very minute. Caps. 3-celled.

252. JUNCUS. Perian. 6-leaved, glumaceous. Caps. superior, 3-celled, 3-valved; cells many-seeded. Seeds attached to a partition in the middle of each ralve.

253. LUZULA. Perian. 6-leaved, glumaceous. Caps. superior, 3-celled, 3-valved; cells 1-seeded, valves without partitions.

\section{TRIGYNIA.}

254. MELANTHIUM. Polygamous.-Cal. o. Cor. 6-parted, rotate; segments unguiculate, with 2 glands at the base of each; claws staminiferous. Caps. subovate, 3-celled; apex partly trifid. Seeds numerous, membranaceously winged.

255. VERATRUM. Polygamous.-Cal. 0. Cor. 6 parted, expanding; segments sessile, without glands. Stam. inserted upon the receptacle. Cap. 3, united, many-seeded.

256. HELONIAS. Cor. 6-parted, spreading, without glands. Styles 3, distinct. Caps. 3-celled, 3horned, cells few-seeded.

257. XEROPHYLLUM. Cor. subrotate, deeply $\vec{U}$ parted. Fil. contiguous at the base. Stig. 3, revolute, partly uniter below. Caps. subglobose, 3-celled; cells 2 -seeded, opening at the summit.

258. TOFIELDIA. Cal. 3-leaved. Pet. 6. Anth. roundish. Styles vertical, short. Caps. 3, superior, united at the base, many-seeded. 
259. SCHEUCHZERIA, Cal.6-parted. Cor. 0. Anth. linear. Stig. sessile, lateral. Caps. inflated, distinct, mostly 2 -seeded.

960. TRIGLOCHIN. Perian. 6-leaved, deciduous ; leaflets concave; 3 of the leaflets inferior and more calycine. Stam. 3-6, very short; anth. turned outward. Stig. nearly sessile, adnate. Caps. 3-6, united above by a com. receptacle and axis, generally separating at the base, 1 seeded, not opening.

261. GYROMIA. Cal. 0. Cor. 5-parted, revolute. Fil. and anth. distinct. Styles 0 ; stig. 3, filiform and divaricate, united at the base. Berry 3celled; cells 5-6-seeded. Sceds compressed, 3 -sided.

262. TRILliUM. Cal. 3-leaved. Cor. 3-petalled. Stig. sessile. Berry superior, 3-celled; cells many-seeded.

-263. RUMEX. Perian. 6-leaved. Nut triquetrous, covered by the 3 interior valviform leares of the perianth. Stig many-cleft.

\section{TETRAGYNIA.}

264. SAURURUS. Flowers in an ament or crowded spike ; sciles 1 flowered. Cor. 0. Anth. adnate to the filaments. Caps. each 1, or rarely 2.seeded.

\section{POLYGYNIA.}

265. Alisma. Cal. 3-leaved. Pet. 3. Caps. numerous, 1 -seeded, not opening.

228. TRADESCAN'TIA. Spider-wort. Commelinea.

1. 'T. virginica: erect; leaves lanceolate, elongated, smooth, flowers in a crowded umbel, sessile, pubescent.

HAB. Shady woods. May. 24. Stem $1 \mathrm{ft}$. high; leav, ranalic.; umb. crozed.; fl. blue. 
2. T. rosea: erect, simple; leaves linear; peduncleelongated; calyx smooth.

HAB. Wet sandy fields. May. 2f. Stem $1 \mathrm{fi}$. high; leav. narrow; fl. small, rose-col.

229. LEONTICE. Berberideœ.

L. thalictroides : leaves bi-triternate ; leaflets 2-3lobed; flowers paniculate, from the centre of the leaves.

HAB. Rocky woods and mountains. Apr.-May. 4. A ft. high, smooth, mostly 2-leav.; fl. sm., greenish-yell.; seed large, dark blue.

230. BERBERIS. Barberry. Berberidea.

B. vulgaris $\delta$. canadensis: branches punctate, armed with trifid spines; leaves oblong-obovate, distinctly ciliate-serrate; racemes simple, subcorymbose. recurved.

HAB. Rocky hills. May-June. Shrub 3-4 ft. high ; leav. altern.; filam. irritable; fl. yell.; ber. red, acid.

231. एRTNOS. Winterberry. Rhamni.

1. P. verticillatus: leaves deciduous, oval, serrate, acuminate, pubescent beneath ; flowers 6-parted, dioecious; staminiferous axillary, subumbellate ; pistilliferous aggregated.

HAB. Moist woods and swamps. June. Shrub 6-3 ft. high; leav. alt.; $f$. white; ber. scarlet.

B. ienuifolius: leares obovate, membranaceous. smooth beneath ; pistilliferous $4-5$ cleft, sübsolitary.

HAB. Swamps. Leav. viviuse, or slightly acum., midrib pubes.

2. P. minigurs: leaves deciduous, oval, cuminate ai each end; flowers 4-cleft; staminiferous ones crowded on the lower branchlets; pistilliferous solitary, on long peduncles.

HAB. Near Philadelphia. A small trec, bark whitish; leav. smooth, petiolate. 
3. P. lcevigatus: leaves deciduous, lanceolate, with adpressed serratures, smooth on both sides, shining above ; nerves beneath scarcely pubescent ; flowers 6-cleft; pistilliferous axillary, solitary, subsessile ; staminiferous scattered.

IIAB. Sivamps. June. Shrub 6-8 ft. high; lear. $2 \frac{1}{2}$ in. long; stam. $f$. pedunc., ber. red.

t. P. glaber: leaves sempervirent, cuneate-lanceolate, coriaceous, smooth and shining, subdentate at the extremity; pedicels axillary, subsolitary, mostly 3-flowered.

IIAB. Swamps and pine-barrens. July. Shrub 3-4 ft. high; fl. perfect? pedunc.; ber. black.

232. FLEERKEA. Juncece?

F. uliginos $\alpha$.

HAB. River marshes. Apr.-May. ๑ Decumb., slend., smooth, leav. alt.; trif. and pinnatif.; pedunc, axill.; fl. small, white.

\section{AMARYLLIS. Amaryilidece.}

1. Atamasco: spath. bifid, acute; flowers pedicellate; corolla subcampanulate, subequal, erect, short and tubular at the base; stamens declined, equal.

HAB. Swamps. June. 24. Root bulb, leav. lin. concave; scape 6 in. high; fl. large, wh. and pink.

234. AlliUM. Onion, \&.c. Asphodelece.

1. A. canadense: scape naked, terete; leaves linear; head bulbiferous.

HAB. Meadows. May. 24. Root bulb.; leav.long, flat above; $f$. numerous, pale rose-col.

2. A. vineale: cauline leaves rounded, fistulous; umbel bulbiferous; stamens alternately tricuspidate.

HAB. Meadows and pastures. May. 2f. Bulb. ovate, stem $2 \mathrm{ft}$.high; leav. long; $f$. deep rose-col. \$.

3. A. triflorum: scape naked, terete, shorter than the leaves; leares lanceolate, nerred, umbel fer. Anwered. 
HAB. Mountains. May-June.

4. A. cernuum: scape naked, quadrangular, umbelliferous; leaves linear, nearly flat; umbel cernuous ; stamens simple; germen 6-toothed.

HAB. Pennsylvania. July 4. Leav. 8-12 in. long, 3-lin. broad; scape 1-2 ft. high; fl. rose-col. 5. A. tricoccum: scape naked, nearly terete; leaves lanceolate-oblong, flat, smooth; umbel globose. seeds solitary.

HAB. Woods and mountains. June-July. 4. Bulb. large; leav. 1 in. broad; fl. white.

\section{HYPOXIS. Star-grass. Asphodelex.}

II. erecta: hairy; scape 2-4-flowered; leaves linear; segments of the corolla lanceolate-oblong.

HAB. Woods and meadows. May-June. 4. Root bulb. solid; leav. gramin.; $f$. yell.

\section{PONTEDEIRIA. Narcissi?}

P. cordata: leaves oblong-cordate; flowers in crowded spikes.

HAB. In fresh water. Aug. 4. Leav. subrad., 3-4 in. long, thick; spath. oblong; fl. aggreg. blue.

3. angustifolia: leaves elongated-triangular, truncate and subcordate at the base.

HAB. Mountain-lakes.

\section{CONOSTYLIS. Homodoracer.}

C. americana: corolla woolly within; scape corymbose-panicled; leaves gramineous-ensiform, glaucous; filaments equal.

HAB. Sandy swamps. July-Aug. 4. Root creep!: scape $1 \mathrm{ft}$. long; $f$. small, yellow within.

\section{ALETRIS. Star-wort. Asphodeler.}

1. A. farinosa: flowers pedicellate, oblong-tubular, corolla, when decaying, nearly smooth; leaves. broad-lancẹolate. 
HAB. Sandy woods. July. 24. Root prcemorse; leav. rad., spreading; scape $2 f t$. high; fl. white.

2. A. aurea: flowers subsessile, short, subcampanulate; corolla, when decaying, rugose and very scabrous ; leaves lanceolate.

HAB. In pine-barrens. July-Aug. 4. scape 2-3 $f t$. high; pedicels bracteate; $f$. yellow.

239. HEMEROCALLIS. Day Lily. Hemerocallidece.

II. fulva: leaves broad-linear, carinate; interior petals obtuse, undulate; exterior nerves of the petals ramose.

HAB. Wet meadows. June-Aug. 4. Leav. very long; scape 3 ft. high; $f$. large, fulvous.

240. AGAVE. Bastard aloe. Bromelia.

A. virginica: stemless, herbaceous; leaves with cartilaginous serratures; scape simple.

HAB. Banks of rivers. Sept. 4. Root promorse; leav. succul.; scape 4-6 ft. high; fl. yellowish.

\section{PHALANGIUM. Asphodelea.}

P. esculentum : root bulbous ; leaves all radical, linear, carinate; stigma minutely 3 -cleft.

HAB. Banks of rivers and lakes. Sept. 44. Root tunic.; scape simp.; $f$. pale blue.

\section{NARTHECIUM. Juncere.}

N. americanum: raceme sometimes interruptedly spiked, lax; pedicels with a setaceous bract below the flower, and another embracing its base; filaments with very short hair.

HAB. Sandy sw:imps. Aug. 4. Root creep.; leav. rad., narrow ensif.; fl. yellow intern.

94. ORNITHOGALUM. Star of Bethlchem. Asphodelece.

O. umbellatum: curymb. few-flowered; peduncles Innger than the bracts; filaments subulate. 
HAB. Moist meadows. May-June. 4. Root bulb.; leav. rad., lin.; fl. wohite. §.

\section{LILIUM. Lily. Liliacece.}

1. L. philadelphicum: leaves verticillate, linear-lanceolate; stem 1-2-flowered : corolla erect, campanulate, spreading; petals unguiculate.

HAB. Copses. June-July. 2f. Stem 2 ft. high: leav. 3-nerv.; $f$. dark orunge.

2. L. canadense : leaves remotely verticillate, lanceolate; nerves hairy beneath; peduncles terminal, elongated, generally by threes; flowers nodding ; corolla turbinate, campanulate, slightly revolute; petals lanceolate.

HAB. Moist meadows. June-July. 24. Stem 2-4 ft. high; $f$ l. about 3, spotted.

3. L. superbum: leaves verticillate, linear-lanceolate, 3-nerved, smooth; the upper ones scattered; flow ers in a pyramidal raceme, reflexed; petals revolute.

HAB. Wet meadows. July. 4. Stem 4-6 ft. high; fl. 3-20, bright orange, spotted.

4. L. Catesbai: leaves scattered, linear-lanceolate: stem 1-flowered; corolla erect; petals with long claws, undulate on the margin, reflexed at the tip. HAB. Pennsylvania. July. 4. Stem $1 \frac{1}{2} \mathrm{ft}$. high; fl. large, scarlet, spotted.

245. ERYTHRONIUN. Dog's-tooth Violet. Liliacer.

1. E. americanum: leaves lanceolate, punctate; petals oblong-lanceolate, obtuse at the point; interior ones bidentate near the base ; style clavate ; stigma entire.

HAB. Shady moist places. April-May. 4. Leav. 2, rad; fl. solit. nod., yellor.

2. E. albidum: leaves elliptical-lanceolate, not punctate; petals linear-lanceolate, obtuse; inner ones without dentures, subunguiculate; style 3 -cleft ; lobes reflexed.

HAB. River banks. April. 4. Leav. 6 in. leng: fl. large, bluish white. 
246. UVULARIA. Bell-wort. Liliacece.

1. U. perfoliata: leaves perfoliate, elliptic-obtuse; corolla campanulate, granular, scabrous within; anthers cuspidate.

HAB. Moist shady places. May-June. 4. 8-10 in. high; fl. pend. pale yell.

9. U. flava : leaves perfoliate, elliptical-oblong, undulate at the base ; corolla tapering at the base, scabrous within; anthers awned.

HAB. Shady woods. May-June. 4. Fl. larger and brighter yellow than $U$. perf.

3. U. grandiflor $\iota$ : leaves perfoliate, oblong, acute ; petals smooth within; anthers without awns; nectaries subrotund.

$\mathrm{HAB}$. Rocky hills. 4. Fl. large, bright yellow.

t. U. sessilifolia: stem smooth; leaves sessile, ovallanceolate, glaucous beneath; petals flat, smooth within ; capsule stipitate.

HAB. Shady thickets. May-June. 24. Stem 6-, $12 \mathrm{in.} \mathrm{high,} \mathrm{forked} \mathrm{above;} f$. pale yellow.

\section{\&47. STREPTOPUS. Liliace⿰.}

1. S. roseus: smooth and shining; leaves amplexicaul, serrulate ciliate, anthers short, 2-horned.

HAB. Mountains. May-June. 4. Stem 18 in. high, dichot.; $f$. rose-col.

2. S. distortus: smooth ; leaves amplexicaul, smooth on the margin; pedicels distorted and geniculate in the middle; anthers sagittate, acuminate, much longer than the filaments.

HAB. Mountain woods. May. 4. Stem 2 fl. high; fl. solit., greenish yellow.

3. S. lanuginosus: hoary-pubescent; leaves sessile, subcordate at the base, acuminate; pedicels by pairs, on a very short foot-stalk.

HAB. High mountains. May. 4. Leav. abrupt, acum. ; fl. large, greenish.

248. CONVALLARJA. Solomon's seal, sc. Liliacer. 
* Corolla deeply 4-parted, spreading; stamens 4 ; berry 2 -celled. (Flowers in a terminal
raceme.) MaJAN THEMUM.

1. C. bifolia: Stem 2-leaved; leaves on short petioles, cordate oblong, very smooth on both sides; raceme simple, terminal ; flowers tetrandrous.

HAB. Shady woouls. May-June. 24. Stem 4-6 in. high; fl. small, white; ber. spotted with rea.

** Cor. 6-parted, spreading; filaments divergent, attached to the base of the segments. (Flowers in a terminul raceme.) SMILACINA.

2. C. stellata: leaves numerous, alternate, oval-lanceolate, amplexicaul ; rareme simple, terminal.

HAB. Wet meadows. May-June. 4. A foot high, terete; fl. small, white.

3. C. trifolia: stem about 3-leaved; leaves alternate, ovare-lanceolate, contracted at the base; raceme simple, terminal, few-flowererl.

HAB. Mountitin swamips May. 4. A span high: fl. sm. white, on short pedicels.

4. C. racemosa: leaves numerous, alternate, sessile, oblong-oval, acuminate, nerved, pubescent ; flowers in a terminal, racemnse panicle.

HAB. Low grounds. June. 4 Stem $18 \mathrm{in.}-2 \mathrm{ft}$. high, subflex.; fl. small, while; ber. red.

*** Corolla subcampanulate, deeply 6-parted; style elongated; berry 2-celled, nuny-seeded.

5. C. umbellulata: subcaulescent: leaves oblong-oval, ciliate on the margin, scape pubescent; umbel. terminal; pedicels nodring, with minute bracts at the base.

HAB. Mountain bogs. May-June. 4. Leav. very large, about 3 , subrud.; scape $6-8$ in. long; umb. $3-4 f$. ; fl. greenish yellow ; ber. blue.

**** Corolla 6-cleft, cylindric; filoments inserted on the upper part of the tube; berry 3-celled; cells 2 - 


\section{seeded. (Flowers axillary.) PoLX- GONATUM.}

b. C. multiflora: stem terete; leaves alternate, am. plexicaul, oblong-oval ; peduncles axillary, manyflowered.

HAB. Rocky hills. June-July. 2f. Stem 2-3 ft. high, smooth ; $f$. gr. white.

7. C. biflora: stem terete, smooth; leaves alternate elliptical-lanceolate, 3-nerved; peduncles axillary, solitary, 2-flowered.

IIAB. Rocky places. 4 . Fl. yellow white, with green lips. + .

8. C. pubescens : stem nearly terete, furrowed; leaves alternate, amplexicaul, ovate, pubescent beneath ; pedancles axillary generally 2 -flowered.

HAB. Rock. June. 4. Stem $18 \mathrm{in}$. high, smooth; fl. yel. wh. and green.

9. C. canaliculata : stem canaliculate; leaves alternate, amplexicaul, oblong, pubescent on the margin; peduncles axillary, 2-flowered.

HAB. Shady woods. 4. t.

10. C. latifolia: stem angular; leaves sessile, ovate, acuminate ; peduncles one or many-flowered.

HAB. Rocky banks. June-July. Stern $3-5 f t$ 。 high; ped. 3-6 fl.; $f$. gr. wh.

249. ASPARAGUS. Aspuragece.

1. officinalis: unarmed; stem herbaceous, erect terete, very much branched ; leaves setaceous, fasciculate, flexible; peluncles jointed in the middle.

HAB. Rocky and gravelly shores. June. 4. F\%. gr. rchite. $\S$.

250. ORONTIUM. Golden club. Aroidea.

O. aquaticum: leaves linceolate-ovate ; scape cylindrical, spiked.

HAB. In water. May. 24. Leav. rad. large; spadix yellow. 
251. ACORUS. Sweet-flag. Aroideo.

A. calamus: spadix protruding from the side of an ensiform leaf.

HAB. Swamps. June. 4. Root creep., arom.; leav. rush-like; spad. greenish.

252. JUNCUS. Rush. Juncece.

* Scapes naked; flozers lateral.

1. J. effusus: scape minutely striate, (soft ;) panicletloose, very much branched, spreading; leaflets of the perianth lanceolate, acuminate, rather longer than the obovate obtuse capsule.

IIAB. Low grounds. June. 4. Scape $2-3 f t$ high; stam. 3 ; seeds attenuatc.

2. J. setaceus: scape filiform, striate, umbel. lateral, compound, few-flowered; peduncles many-flowered; leaflets of the perianth subulate.

HAB. Swamps. 4. Scape slend., 2 ft. high; pan. small; caps. acute.

3. J. acutus: scape naked, terete, panicle lateral; involucrum 2-leaved, spinous; capsule nearly round, mucronate, as long again as the perianth.

HAB. Sandy sea-coast. 4. Stern in subdistich. fascic., 2-3 ft. high; seeds ang.

* Leaves all radical ; (flowers terminal.)

1. J. squarrosus: leaves setaceous, (rigid,) 'grooved; panicle terminal, elongated, compound; capsules elliptical.

HAB. New-York. + .

5. J. tenuis: stem erect, filiform, a little dichotomous at the summit, nearly terete; leaves setaceous, canaliculate; flowers solitary, approximate, subsessile; perianth longer than the obtuse capsule.

HAB. Wet or dry places. June-July. 4. A foot high, tenacious; pan. subcorymb.

6. J. nodosus: stem somewhat leafy ; leaves nodosearticulate; heads mostly 2 , globose, one of them lateral and pedunculate, the other sessile; leaflets 
of the perianth mucronate, shorter than the acu. minate capsule.

HAB. Wet places. July. 4. Stem 8-10 in. high : heads 8-12 fl., 1 sess.

\section{*** Stems leafy.}

$\dagger$ Leaves nearly plane, grooved above.

\%. J. marginatus: leaves flat, smooth ; corymb terminal. simple, proliferous; heads 5-10-flowered; flowers triandrous; leaflets of the perianth about as long as the obtuse capsule ; the exterior ones and the bracts subaristate.

IIAB. Low grounds. Aug. 4. Stem 12-18 in. high ; leav. mostly rad.; stam. 3.

8. J. trifidus: sheaths ciliate; those at the base of the stem leafless; bracts foliaceous, very long, grooved; heads about 3 -flowered, terminal, stem 1-leaved. HAB. High mountains. Aug. 4. Six in. high; leaf nearly term., setac.

9. J. bulbosus : stem simple leafy, compressed ; leaves linear-setaceous, canaliculate; panicle terminal, compound, subcymose, shorter than the involucrum; leaflets of the perianth incurved, obtuse or acute, generally shorter than the ovate, subglobose capsule.

HAB. Salt marshes. Aug.-Sept. 4. A foot high. wiry; caps. dark browen.

10. J. bufonius: stem dichotomous above, paniculate; leaves filiform, setaceous, canaliculate; flowers subsolitary, sessile, unilateral, leaves of the perianth very acuminate, much longer tban the ellipti cal ovate capsule.

HAB. Wet places. June-Aug. ๑. Stem 3-6 in. high; sheaths membran.

\section{t† Leares rounded or subcompressed.}

11. J. acuminatus: stem leafy, erect; leaves terete. nodose-articulate; panicle terminal, compound ; heads 3-6-flowered, pedunculate and sessile: 
leatlets of the perianth linear-lanceolate, subaristate, shorter than the acute capsule.

HAB. Bogs. Aug. 4. Stem $18 \mathrm{in.} \mathrm{high,} \mathrm{tenac.;}$ caps. triquet.

12. J. polycephalus : stem leafy, erect; leaves compressed, nodose-articulate; panicle decompound; heads globose, many-flowered; flowers triandrous; leaflets of the perianth subaristate, rather shorter than the triquetrous acute capsule.

HAB. Bogs. Aug.-Sept. 4. Stem 13 in.-2fi. high, subcompress. belore; heads 12-15-fl. stam. 3, (rarely 6.)

13. J. subverticillatus: Cauline leaves subulate, nodosearticulate; panicle corymbose; heads about 5flowered, fasciculate-verticillate ; leaflets of the perianth striate, as long as the obtuse capsule.

I1AB. Bogs. July. 4. Stem 8-12 in. long, slend.; heads $3-5 \mathrm{fl}$.

\section{LUZULA. Juncere}

1. L. pilosa: leaves hairy; panicle subcymose; peduncles 1 -Howered, reflexed; leaflets of the perianth acuminate, rather shorter than the obtuse capsule.

HAB. Mountains. Apr.-May. 4. Stem 6 in. high, slend.; pedic. capill.

2. L. campestris: leaves hairy; spikes sessile and pedunculate; leaflets of the perianth acuminate, longer than the obtuse capsule.

HAB. Low grounds and woods. Apr.-May. A foot high; spik. roundish.

3. L. melanocarpa: culm leafy ; leaves sublanceolate, smooth; panicles capillary, loose; fowers solitary, distinctly perlicellate; capsules black.

HAB. High Mountains. July. 2f. A foot high; pedicels solit.; caps. oblong.

1. I. spicata: leaves narrow, hairy at the throat; spike cernuous, compound; leaflets of the perianth acuminate-aristate, about as long as the roundish capsule.

HAB. High Mountains. Aug. 24. A foothigh; spikelets bract. ferrug. 


\section{TRIGYNIA.}

254. MELANTHIUM. Nelanthacere.

1. M. virginicum: panicle pyramidal, petals oval, somewhat hastate, flat ; flowers mostly perfect.

HAB. Wet Meadows. July-Aug. 4. Stem $3 f t$. high; pan.large, loose; fl. polygamo-dioic., greenish wh., at length brown.

2. M. dioicum: root a tunicated bulb ; leaves glaucous, gramineous, márgined ; raceme mostly simple, few-flowered ; flowers perfect ; petals roundish, unguiculate, with two spots ; seeds subulately winged.

IIAB. Banks of Rivers. July-Aug. A foot high, $f$. whitish.

3. M. hybridum: panicle racemose, petals suborbicular, plaited, with long claws ; glands connate.

HAB. Pennsylvania. Stem 2 ft. high; leav. long-lin. fl. not changing.

955. VERATRUM. Green and White Hellebore. Melanthacese.

$\mathrm{V}$ : viride : racemes paniculate ; bracts of the branches oblong-lanceolate; partial ones longer than the subpubescent peduncles; leaves broad-ovate, plicate.

IIAB. Swamps. June-July. Root large; stem 3-4 ft. high; fl.green.

\section{HELONIAS. Miclanthacex.}

1. H. latifolia : scape leafless; spike ovate, crowded; bracts linear-lanceolate, leaves lanceolate, mucronate, nerved.

HAB. Swamps. May. Fl. pale purp.; anth. bluc.

2. H. erythrosperina : scape leafy ; leaves linear, very long; raceme oblong; bracts short; capsule shortened, with divaricate horns; seeds ovate, red.

IIAB. Shadly river banks and mountains. June. If . 
Root bulb.; stem 2 ft. high, fl: wh.; seeds with a scarlet coat.

3. H. angustifolia: scape leafy ; leaves very long and narrow; raceme oblong, lat. ; capsule oblong; seeds linear.

HAB. New-York. Root fibr.; stem $2 f t$. high; racem. simp.; $f$. wh.

1. H. dioica: scape leafy; racemes dioicous, spiked, cernuous; pedicels very short, without bracts : petals linear; stamens exserted; leaves lanceolate.

IIAB. Wet meadows. June. Root promorse, stem 1-2 ft. high; $f$. white.

\section{XEROPHYLLUM. Melanthaceæ.}

X. setifolium: raceme oblong, crowded; bracts setaceous; flaments dilated at the base, as long as the corolla; leaves subulate setaceous.

HAB. Pine barrens. June. 4. Root subbulb.; rad. leav. long, cespit.; fl. white.

\section{TOFIELDIA. Melanthacea.}

T. pubens: flowers on a scape, rachis and pedicels scabrous; spike oblong, interrupted; capsules subglobose, scarcely longer than the calyx.

HAB. Delaware. 4. Leav. narrow-ensif., subradic.: scape $18 \mathrm{in}$. high; spike gr. wh.

259. SCHEUCHZERIA. Juncaginea.

S. palustris.

IIAB. Swamps. 2f. July. Root horiz. stem 3-12 in. high; leav. lin.; racem. $5-7$ f.; $f$. peduncul. gr. yell.; caps. large.

260. TRIGLOCHIN. Arrow-grass. Juncaginea.

1. T. maritimum: fruit ovate-oblong, of 6 united capsules.

HAB. Salt marshes and about salt springs. July- 
Aug. 24. Leav. rad., narrow, rush like; spike very long.

2. T. palustre : flowers triandrous ; fruit of 3 united capsules, nearly linear, attenuate at the base.

HAB. Marshes. July. 4. Leav. very narrow ; scape slend. ; 12 in. high.

961. GYROMIA. Indian Cucumber. Asparagece.

G. virginica.

HAB. Moist woods. May-June. 4. Root tub., stem simp.; leav. verticill.; fl. yell.

262. TRILliUir. American Herb Paris. Asparagece.

1. T. sessile: flower sessile, erect ; petals lanceolate, twice as long as the calyx ; leaves sessile, broad oval, acute.

IIIB. Shady rocks. 4. Root prcemorse, and fib.; $f$. and ber. purp.

2. T. erythrocarpum: peduncles somewhat erect; petals oval-lanceolate, acute, recurved, nearly as long again as the narrow calyx; leaves ovate, acuminate, rounded at the base; abruptly contracted into a short petiole.

HAB. Sphagnous swamps. May. 4. Stem 8 in. high; fl. wh. with purp. veins.

3. T. pusilluun: peduncle erect; petals scarcely longer than the calyx ; leaves oval oblong, obtuse, sessile.

IIAB. Pennsylvania. Plant small; pet. pale flesh col.

1. T. cernuum : peduncle recurverl; petals lanceolate, acuminate, flat, recurved, of the length and breadth of the calyx ; leaves dilated-rhomboid, abruptly acuminate, on short petioles.

HAB. Shaily woods. May. H. Stem 12-18 in. high ; $f$. sh. ; ber. lurge, purp.

$\therefore \mathrm{T}$. erectum: peduncle inclined; flower nodding; petals ovate. acuminate, flit, spreiding, broader and a little longer than the calyx ; leaves broulrhombnirl, acuminate, sessile. 
HAB. Rich soil, among rocks. May. 4. Aft.high : fl. large, dark purp.

B. album: flowers smaller, petals white.

6. T. pendulum: peluncle inclined; flower pendulous ; petals ovate, shortly acuminate, flat, spreading, nearly equal to the ovate acuminate culyx ; leaves roundish-rhomboid; acuminate subsessile.

HAB. Mountains. May. 4. A foot high; leav. acute at base; $f$. wh. veined.

7. T. grandiflorum: peduncle inclined; flower somewhat erect; petals spatbulate-lanceolate, connivent at the base, much longer than the calyx; leaves broadly rhomboid-ovate.

HAB. Rocky woods. 4. May. Stem. 8-10 in. high; leav. sess; fl. large, wh.

263. RUMEX. Dock. Polygonece.

* Flowers all perfect; valves graniferous. LAPATHUM.

$\uparrow$ Valves entire.

1. F. aquaticus: valves ovate, entire, all of them graniferous; leaves lanceolate, all of them cordate at the base.

HAB. IVet places. June. Root large, astrin.; stem $3-4 \mathrm{ft}$. high; grains linear. $\S$ ?

?. R. crispus: valves very large, cordate, entire, reticulate, graniferous ; leaves lanceolate, undulate, acute.

HAB. Meadows. June. Root yell.; stem $\cong-3 f t$. ligh; grains unequal, $\S$.

i. R. sanguineus : valves oblong, small, one of theus graniferous ; leaves lanceolate, subcordate.

HAB. Fields. June. Stern 2-3 ft. high; lear. mostly varieg. reith red, $\S$.

4. R. Britannica: valves all entire and graniferous; whorls of flowers leafless; leaves broad-lanceolate, flat, smooth ; sheaths obsclete.

HAB. Wet places. June. Root large, dark ext., yell. int.; stem 2-3 ft. high.

5. F. rerticillatus : valves cntire, all of them granife- 
rous ; racemes leafless ; leaves lanceolate ; sheaths cylindrical.

HAB. Wet places. June. Root large; stem $2 \mathrm{ft}$. high ; fl. semiverticil.

\section{$\dagger \dagger$ Valves toothed.}

C. R. acutus: valves oblong, somewhat toothed, all of them graniferous ; leaves cordate-oblong, acuminate, whorls leafy.

HAB. Waste places. May. Stem $2-3 f t$. high ; low'er leav. large, §.

7. R. obtusifolius : valves dentate, one of them conspicuously graniferous; radical leares ovate-cordate, obtuse ; stem somewhat scabrous.

HAB. Woods and fields. June-July. Root brown ext., yell. int.; rad. leav. very large, §.

* Floriers diccious; valves grainless. ACETOSA.

3. R. Acelosella: leaves lanceolate-hastate, with the lobes spreading or recurved.

HAB. Fields. May-July. Stem 4-12 in. high; plant acid. §. Pist. Fl. rare.

\section{ITETRAGYNIA.}

264. SAURURUS. Lizard's-tail. Najades?

S. cernuus.

HAB. In water. July-Aug. 4. Stem $18 \mathrm{in.}-2 \mathrm{ft}$. high; leav. alt., petiol. cordate, spike 3-6 in. long, sihite, cal. tub.

\section{POLYGYNIA.}

265. ALISMA. Water-Plantain. Alismacece.

1. Plantago: leaves ovate-cordate, acute or obtuse, 9 -nerved; flowers in a compound verticillate panicle; fruit obtusely triangular.

HAB. In water. July-Aug. 4. Leav. rad. ?nerv. : petiol.; pan. large; $f$. reh.

3. parviflora: leaves oval, 5-7-nerved, acuminate ; Howers very sinall. 


\section{HEPTANDRIA.}

\section{MONOGYNIA.}

266. TRIENTALIS. Cal. 7-leaved. Cor. 7-parted. equal, flat. Berry dry, 1-celled, many-seeded. 367. ESCULUS. Cal. 1-leaved, 4-5-tonthed, ventricose. Cor. 4-5-petailed. Pet. unequal, pu= bescent, inserted upon the calyx. Caps. 3 celled. Seeds large, solitary.

\section{MONOGYNIA.}

266. TRIENTALIS. Chick-weed winter-green. Primulacecr.

T. americana : leaves narrow-lanceolate, acuminate, serrulate ; petals acuminate.*

HAB. Woods and swamps. 4. May-June. Leav. zohorled; fl. sol., woh.

267. ESCULUS. Horse-chesnut. Aceracece.

A. glabra: leaves quinate, very smooth, corolla 4petalled, spreading, with the claws as long as the calyx ; stamens longer than the corolla; fruit spinous.

HAB. Woods. May. Ђ. A large shrub; fl. large, yel. r:h., panicul.

\section{OCTANDRIA.}

\section{MONOGYNIA.}

* Flowers superior.

268. RHEXIA. Cal. urceolate, 4-5-cleft. Pet. 4, inserted upon the calyx. Anth. incumbent, attached to the filaments behind, naked at the base. Caps. setigerous, 4-celled, free in the ven-

* These characters, pointed out by Dr. Bigelow, I am now satistied, will distinguish the N. American Trientalis from the European. 
tricose calyx. Recept. lunulate, pedicellate. Seeds numerous.

269. CENOTHERA. Cal. tubular, 4-cleft; segments deflexed, deciduous. Pet. 4. Stig. 4-cleft. Caps. 4-celled, 4-valved. Seeds naked, affixed to a central, 4-sided receptacle.

270. GAURA. Cal. 4-cleft, tubular. Cor. 4-petalled, ascending. Nut quadrangular 1 -seeded.

271. EPILOBIUM. Cal 4-cleft, tubular. Cor. 4-petalled. Caps. oblong, inferior. Seeds comose.

272. OXYCOCCUS. Cal. superior, 4-toothed. Cor. 4-parted; segments sublinear, revolute. Filam. connivent; Anth. tubular, 2-parted. Berry many-seeded.

* Flozers inferior.

273. MENZIESIA. Cal. 1-leaved. Cor. monopetalous, ovate. Filam. inserted into the receptacle; anth. awnless. Caps. superior, 4-celled; dissepiments formed by the inflexed margins of the valves. Seeds numerous, oblong.

274. ACER. Flowers mostly polygamous. Cal. 5cleft. Pet. 5 or 0 . Sumarce 2, winged, united at the base, by abortion 1 -seeded.

275. DIRCA. Cal. 0. Cor. tubular; border obsolete. Stam. unequal, exserted. Berry 1 -seeded.

276. JEFFERSONIA. Cal. 4-5-leaved. Cor. 8-petalled. Caps. obovate, substipitate 1-celled, opening below the summit by a semicircular foramen. Seeds numerous, arillate at the base.

\section{TRIGYNIA.}

277. POLYGONUM. Perianth 5-parted, petaloid, inferior. Nut 1-seeded, mostly angular.

\section{MONOGYNIA.}

268. RHEXIA. Melastomacea.

1. R. virginica: stem with winged angles, somewhat hairy; leaves sessile, ovate-lanceolate, serrite- 
ciliate, sprinkled with appressed hairs on both sides.

HAB. Wet meadows. July-Aug. 4. Stem quad. $1 \mathrm{ft}$. high ; fl. large, purp.

2. R. mariana: very hairy; leaves lanceolate-acute at each end, 3-nerved, subpetiolate ; calyx tubular, nearly smooth.

HAB. Bogs and wet wonds. Aug. 4. A foot high; stem subterete; fl. purp.

3. R. ciliosa: stem subquadrangular, smooth; leaves subpetiolate, oval, smooth beneath, slightly hispid above; margin serrulate-ciliate; flowers involucrate.

HAB. Delaware. A foot high, slend. ; leav. small ; $f$, purp.; pet. roundish.

269. OENOTHERA. Night willow-herb. Onagrarice. * Capsule elongated, sessile.

1. E. biennis: stem villous and scabrous; leaves ovate-lanceolate, flat, dentate; flowers somewhat spiked, sessile: stamens shorter than the corolla. HAB. Fields. June-Oct. $\odot$ s. Stern $3-5 \mathrm{ft}$. high; leav. alt. ; fl. racem., yellow.

2. OE. muricata: stem purplish, muricate; leaves lanceolate, flat; stamens longer than the corolla.

HAB. Fields. Fl. smaller.

3. E. parviflora: stem smooth, subvillous; leaves ovate-lanceolate, flat; stamens longer than the corolla.

$\mathrm{HAB}$. Fields and woods. $8 .+$

4. E. grandiflora: stem nearly smooth, branched; leaves ovate-lanceolate, smooth; stamens declinate.

IIAB. Fields. July-Aug. đ. Stem 3-5 ft. high, tercte; $f$. large, ycllow.

5. QE. sinuata: stem diffused, pubescent; leaves ovaloblong, sinuate-dentate ; flowers axillary, villous'; capsule prismatic.

6. minima: stem humble, simple, 1-flowered: leares entire. 
HAB. Sandy fields. ๑. 1-3 in. high, 1-2 fl. small, sess.; caps. lin.

** Capsule ventricose, angular, mostly pedicellate.

6. E. fruticosa : somewhat villous; leaves linear-lanceolate, subdentate; acute; petals broad-obcordate; capsules oblong-clavate, pedicellate, quadrangular; raceme naked below.

HAB. Hills and woods. June. ๑. Stem 12-18 in. high, purp. ; leav. punc.

3. ambigua: more or less pilose; stem simple; leaves lanceolate or ovate-lanceolate, acute, subdenticulate; petals obcordate, longer than broad; points of the calyx very short; capsules subsessile, always smooth, oblong and 4-winged; raceme naked below.

HAB. Hills and dry woods. July. Leaves $2 \frac{1}{2}$ in. long.

7. CE. incana: stem slender, erect; leaves flat, hoary and tomentose, very entire, elliptic-ovate, acute; raceme few-flowered, naked; capsules subsessile, oblong and quadrangular.

HAB. Dry Woods. Stem 6-8 in. high; $f$. bright yellow. +.

8. QE. pumila: smooth; stem ascending; leaves lanceolate, very entire, obtuse; capsule subsessile, elliptical-obovate, angular.

IIAB. Dry fields. June. 4. A span high: $A$. small; pet. obcordate.

9. E. chrysantha: stem slender, pubescent; leaves lanceolate, rather obtuse, flat, entire; segments of the calyx as long again as the tube; capsule clavate, sessile, acutely angular.

IIAB. Mountains. A foot high; $f$. small, bright yellow.

10. C. pusilla: subpubescent; stem nearly simple; leaves lanceolate-oblong, somewhat obtuse, entire; flowers axillary at the summit; capsule sessile, clavite-turbinate, almost equally 8 -angled. IIAB. Mountains. Stem 4-6 in. high, erect. 


\section{GAURA. Onagrarice.}

G. biennis : leaves lanceolate, dentate ; spikes crowded ; fruit roundish, subquadrangular, pubescent.

HAB. Banks of rivers. Aug. ¿ै. Stem $1 \frac{1}{2}-2 f t$. high, hairy; $f$. num. rose-col.

\section{EPILOBIUM. Willow-herb. Onagrarice.}

1. E. spicatum: leaves scattered, veined, smooth; flowers subspicate; stamens declinate.

HAB. Swamps. Aug. iे. Stem $3-5 \mathrm{ft}$. high, terete; cal. col.; fl. large purp.

2. E. coloratum : stem terete, pubescent ; leaves lanceolate, serrulate, petiolate, opposite, smooth, with coloured veins; the upper ones alternate.

HAB. Wet places. July-Aug. 4. Stem 3-4 $f$. high; upper bran. subquadran.; fl. axill. purp.; caps. $2-3$ in. long.

3. E. rosmarinifolium : stem terete, pubescent, branching above ; leaves linear, tery entire; those on the stem opposite ; on the branches alternate ; flowers pedunculate; petals bifid; stigma entire. HAB. Swamps. Aug. 4. Root bulb. and scaly; $f$. very small, pale purp.

1. E. molle: densely and softly pubescent; stem terete; leaves oblong linear, sessile, very entire ; the lower ones opposite; upper ones alternate ; flowers pedicellate, subterminal, regular; petals 2-lobed ; stigma entire.

IIAB. Wet grounds. Aug. 4. Stem $18 \mathrm{in.} \mathrm{high,}$ !. branch. above; silky pubes.; fl. small, pale purp.

272. OXYCOCCUS. Cranberry. Vaccinece.

1. O. macrocarpus: creeping; branches ascending; leaves oblong, nearly flat, obtuse, with distant obsolete serratures, glaucous beneath; pedicels elongated; segments of the corolla lincar-lancenlate. 
HAB. Sphagnous swamps. June. 4. Stem long, filif.; fl. red; ber. scarl.

\$. O. vulguris: leares ovate, entire, revolute; segments of the corolla oval ; stem filiform, creeping, naked.

HAB. Mountain bogs. 4. +.

\section{MENZIESIA. Ericece.}

1. M. ccrulea: leaves scattered, crowded, linear, toothed ; pedicels terminal, aggregate, 1 -flowered; flowers campanulate, decandrous, 1-flowered, calyx very acute.

HAB. High mountains. July. 万. Evergreen, branch.; fl. large, purp.

2. M. globuluris : leaves lanceolate, glaucous beneath: except the nerves, pubescent; calyx 4-cleft ; flowers globose, octandrous.

HAB. Mountains. June. $h_{2}$. Stem 4 in. high; $f$ t yell. brown.

\section{ACER. Maple. Aceracece.}

1. A. rubrum: leaves palmately about 5-lobed, cordate at the base, unequally and incisely toothed, glaucous beneath; the sinuses acute; flowers aggregated in about fives, on rather long pedicels; germens glabrous.

HAB. Wouds. April. A large tree; precocious; $f$. red; stam. 5-6.

2. A. dasycarpon: leaves palmately 5-lobed; truncate at the base, incisely toothed, smooth and whitish beneath ; sinuses obtuse ; pedicels short ; germens tomentose.

HAB. Banks of rivers. April. Alarge tree; $f . g r$. yel.: pet. 0 ; fruit large.

3. A. barbatum: leaves ovate-cordate, with 3 short lobes, unequally serrate, glaucous beneatl, and pubescent on the nerves; pelluncles hairy; those of the staminiferous flower branched: of the pistilliterous simple ; wings of the samaræ erect. HAB. Cedar swamps. A small tree; leav. small; $A$. pale green. + 
4. A. saccharinum: leares palmately 5-lobed, subcordate at the base, acuminate, glaucous beneath; peduncles corymbose, nodding.

HAB. Woods. April. A large tree; $f$. yellowish on filif. ped.; wings narrow.

5. A. nigrum: leaves palmately 5-lobed, cordate, with the sinus closed; lobes divaricate, sinuate-subdentate, pubescent beneath ; flowers corymbed ; samaræ turgid, subglobose.

HAB. Mountainous regions. April. A large tree; $f$. yellowish on filif. ped., apet.

i. A. pennsylvanicum: leaves with 3 acuminate lobes, rounded at the base, acutely dentate, smooth; raceme simple, pendulous.

HAB. Mountains. May. Shrub ten ft. high; bark striped; fl. gr. yel., large.

7. A. montanum: leaves somewhat 5-lobed, acute, dentate, pubescent beneath; racemes compound, erect.

HAB. Mountains. May. Shrub 6-10 ft. high; lear. sinall ; $f$. greenish.

+ Flowers dicecious. Calyx minute, 5-toothed. Petals 0. stamens 5 ; anthers linear, sessile, acuminate. Samarce as in Acer.-NEGUNDIUM.

8. A. Negundo: leaves pinnate and ternate, unequally serrate.

HAB. River banks. April. A large tree; leaflets 5-7; racem. pend.

275. DIRCA. Leather-wood. Thymelea.

D. palustris :

HAB. Woods. April. Shrub 2 fl. high, with tought bran.; $f$. and bark yellow.

276. JEFFERSONIA. Podophyllacece.

T. diphylla.

HAB. Fertile woods. 24. Leaflinate, petiol.; scape $1 \mathrm{ft}$. high, fl. sol. term., large, white; caps. large, coriac. 


\section{TRIGYNIA.}

277. POLYGONUM. Persicaria, \&c. Polygonece.

* Floxers axillary.

1. P. aviculare: stamens 8 , styles 3 , leaves lanceolate, scabrous on the margin ; nerves of the stipules distant ; stem procumbent, herbaceous.

HAB. Fields, \&c. May-Oct. ๑. Much bran.; f. very small, wh. or reddish: seed striate.

2. P. glaucum: flowers octandrous; styles 3 ; leaves lanceolate, thick and glaucous, revolute on the margin ; stipules lacerate ; pedicels exserted; stem diffuse, procumbent ; seed acutely angular, smooth and shining.

HAB. Sandy sea-shore. Aug. $\odot$ ? Stem long, sublig. ; fl. larger then No. 1, rose-col.

3. P. tenue: stem slender, erect, branched, acutely angular; flowers alternate, subsolitary; leaves linear, acuminate, straight ; stipules tubular, lacerate, with the segments finely attenuate at the extremity.

HAB. Rocks and sandy fields. July-Sept. ๑ Stem 6-10 in. high; ang. scab.

\section{** Flowers spiked.}

1. Spike solitary, terminal; stamens; stigmas 3 , nut triangular. Bistortan

4. P. viviparum: stem simple; spike linear, solitary ; leaves linear-lanceolate, revolute on the margin; the lower ones elliptical, petiolate.

HAB. High mountains. Aug. 4. Stem 6-8 in. high; stip. tub., smooth; fl. red.

I† Spikes axillary or terminal; stamens $5-8$; stigmas mosily 2 ; nut ovate. Persicaria.

j. P. punctatum: flowers octandrous, glandular-punctate; styles 3-parted; stipules slightly hairy, ciliate ; spike tiliform, at first cernuous ; leaves lanceolate, with pellucid punctures, scabrous on the margin and midrib. 
HAB. Wet places. Aug.-Oct. 4. Stem $1 \frac{1}{2} \mathrm{ft}$. high; $f$. wh.-plant acrid.

6. P. mite: flowers octandrous, somewhat crowded ; styles 3-parted; leaves narrow-lanceolate, somewhat hairy ; stipules hairy, with long ciliæ.

HAB. Wet places. Aug.-Sept. 4. Stem $18 \mathrm{in.}$ high, fl. pale red or white; plant not acrid.

\%. P. virginianum: flowers 4-cleft, unequal, remote, pentandrous; styles 2 ; spike very long, virgate; leaves oval-lancenlate.

HAB. Moist sbady places. Aug.-Sept. 4. Stem 2-4ft. high, $f$. white; fr. birost.

8. P. amphibium: flowers pentandrous; styles bifid; spike oblong or ovate; leaves petiolate, oblong or lanceolate, subcordate.

a. terrestre : stem nearly erect; leaves oblong-lanceolate, often cordate at the base, smooth above, slightly pubescent beneath; spike ovate-oblong.

HAB. Borders of ponds. Aug. 4. Stem assurg., 8 in. high; spike bright rose-col.

3. aquaticum: leaves floating, ovate-lanceolate; spike cylindrical-oblong.

HAB. Floating in lakes. Aug. 4. Stem 3-10 ft. long, branch.; ff. rose-col.

3. P. pennsylvanicum: flowers octandrous; style 2cleft; spike oblong, crowded; peduncles hispid; leaves lanceolate, slightly hairy; stipules smooth and naked.

$\mathrm{HAB}$. Fields, and along ditches. July-Oct. $\odot$ Stem 2-4 ft. high; leav. pale gr. ; fl. red.

10. P. lapathifolium: flowers hexandrous; styles 2 ; spikes oblong, rather crowded, erect; peduncles. scabrous; leaves ovate-lanceolate, on short petioles, hoary.

HAR. Wet plares. Aug. ๑. Stem 2-4 ft. high, leav. pale green.

11. P. Persicaria : flowers hexandrous: styles bifid; spikes ovate-oblong. erect; peduncles smooth; leaves lanceolate; stipules smooth, ciliate.

HAB. Low grounds. July-Aug. ○. Stem 1--2 ft.high; spikes dense, rose-col. 
12. P. orientale : flowers heptandrous, digynous, leaves ovate; stem erect; stipules hairy, hypocrateriform.

HAB. Road sides, \&c. Aug.-Sept. ○. Stem 35 ft. high, pubes.; spik. subpend, rad.

*** Flowers in paniculate spikes; perianth 5-leaved. Polygonella.

13. P. articulatum: flowers perfect, octandrous, trigy. nous, nordding; spikes paniculate, filiform; pedicels solitary, articulate near the base; bracts im. bricate; leaves linear; nut triquetrous.

HAB. Barren sandy woods. Sept. $\odot$. A foot high, branched; bracts trunc. ; $f$. rose-col.

***** Flozers in racernose panicles; (leaves subcordate or sagiticte.) FAGOPYRUM.

14. P. Convolvulus : flowers octandrous; styles 3-cleft ; leaves oblong, hastate-cordate; stem climbing, angular, somewhat scabrous ; segments of the perianth obtusely carinate.

HAB. Sandy fields, \&c. July-Sept. ๑. Leav. petiol.; raceme interrupt.; $f$. reddish.

15. P. cilinode: flowers octandrous; styles 3-cleft; leaves cordate; stipules rither acute, ciliate at the base; stem angular, climbing or prostrate, pubescent; segments of the perianth obtusely carinate.

HIAB. Hills. Aug. ๑. Plant minutely pubes.;

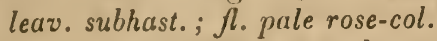

16. P. scandens : flowers octandrous, trigynous; leaves broadly cordate; stipules truncate, naked; stern climbing, smooth; segments of the perianth winged.

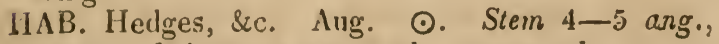
purp.; fl. large, aggreg. wh. or rose-col.

67. P. sagittatum: llowers octandrous, capitate; styles 3-cleft; leaves sagittate, stem retrorsely aculeate. HAB. Wet thickets. July-Aug. ๑. Stem slend., prost.; $f$. in sm. heads, white.

18. P. arifolium: flowers hexandrous, distinct; styles bifid; spikes few-flowered, leaves hastate; stem retrorsely aculeate. 
HAB. Wet thickets. July-Sept. ○. Stem slend., prost., remotely acul.; fl. rose-col.

\section{ENNFANDRIA. MONOGYNIA.}

278. LAURUS. Cal. mostly 6-parted, petaloid. Neci. consisting of 3 bisetose glands surrounding the germen. Stam. 12; 6 of them interior, 3 of which are sterile and glanduliferous.

\section{MONOGYNIA.}

LAURUS. Sassafras, \&c. Laurinece.

1. L. carolinensis: leaves perennial, oval-lanceolate, coriaceous, glaucous beneath; peduncle simple. terminated by a fascicle of few flowers; exterior segments half as long as the interior.

HAB. Swamps of Cupressus disticha.-A small tree; leav. ent.; $f$ l. clust., yellore.

\section{* Euosmus.}

Flowers polygamous or diøecious. Cal. 6-parted. Nect. 0. Stam. 9, fertile; 6 exterior naked; the 8 interior augmented by 6 infertile ones attached by pairs; anth. of the sterile stam. glanduloid. Berry 1-seeded. (Leaves deciduous.)

2. L. Benzoin : flowers in conglomerate umbels, diœcious ; buds and pedicels smooth; segments cuneate-oboval, entire, whitish and subpubescent beneath.

HAB. Shady wet places. April. An arom. shrul 4-10 ft. high, fl. yellow; berry scarl.

3. L. Sassafras; flowers in conglomerate corymbs, diœcious ; buds, younger branches, and under sur-

- face of the leaves pubescent ; leaves entire, or 23-lobed; under surface prominently veined.

HAB. Woods. April. A middle sized tree; ft. gr. yellow; ber. blue, on red ped. 


\section{DECANDRIA.}

\section{MONOGYNIA.}

* Flowers menopetalous.

279. ARBUTUS. Cal minite, 5-parted. Cor ovate, diaphanous at the base; border small, 5-cleft, revolute. Filam. hairy. Berry superior, 5celled.

280. GAULTHERIA. Cal. 5-cleft, with 2 bracts at the base. Cor ovate ; border small, 5-cleft, revolute. Filam. hairy. Recept. 10-toothed. Caps. superior, 5 -celled, covered by the calyx, which becomes a berry.

91. VACCINIUM. Cal. superior, 4-5-toothed. Cor. urceolate or campanulate, 4-5-cleft. Filam. inserted upon the germen. Berry 4-5.celled。 many-seeded.

982. ANDROMEDA. Cal. 5-parted. Cor ovate, or subcylindrical; border 5-cleft, reflexed. Anth. 2-horned. Caps. 5-celled. 5-valved; dissepiments from the middle of the valves.

283. KALMIA. Cal. 5-parted. Cor. hypocrateriform; border on the under side, producing 10 cornute protuberances, in which the anthers are concealed. Caps. 5-celled, many-seeded; dissepiments marginal.

984. RHODODENDRON. Cal. 5-parted. Cor. subinfundibuliform, 5-cleft. Stam. 5-10, declinate; anth. opening by 2 terminal pores. Caps. 5-celled, 5-valved, opening at the summit.

285. RHODORA. Cal. 5-toothed. Cor. 3-petalled; petals unequal, slightly united at the base; the upper one thrice broader, and 3-lobed. Stam. and style declinate. Caps. 5-celled, 5valved, opening at the top; dissepiments formed of the inflexed margins of the valves.

286. EPIGÆA. Cal. 5-parted, with 3 bracts at the base. Cor. hypocrateriform; border 5-parted, spreading; tube villous within. Caps. 5-celled. receptacle 5-parted. 
287. PTEROSPORA. Cal. 5-parted. Cor. monopetalous, ovate, margin 5-toothed, reflexed. Anth. excentrically peltate, 1 -celled, bisetose. Caps. 5-celled, imperfectly 5-valved; dissepiments from the middle of the valves; septa and valves uniting toward the base, and coalescing with the receptacular axis. Seeds very minute and numerous, each furnished with a terminal wing.

\section{** Flowers polypetalous, regular.}

288. MONOTROPA. Cal. 3-5-parted, or 0. Cor. 5-petalled, cucullate at the base. Anth. 1-celled, bilabiate. Caps. 5-celled, 5-valved. Seeds namerous, invested with a long arillus.

289. PYROLA. Cal. small, 5-cleft. Pet. slightly united at the base, deciduous. Stam. opening with 2 pores. Cups. 5-celled, 5-valved. Seeds invested with a long arillus.

290. LEDUM. Cal. minute, 5-toothed. Cor. 5-petalled, spreading. Stam. exserted; anth. opening by 2 terminal pores. Caps. subovate, 5-celled, 5 -valved, opening at the base; valves with the margins inflexed and approximate; recept. 5lobed; colum. 5-angled, pedicellate. Seeds numerous, flat, linear, scabrous, with a membranaceous wing at each extremity.

291. LEIOPHYLLUM. Cal. deeply 5-parted, persistent. Cor. 5-petalled. Stam. longer than the corolla; anth. lateral, opening on the inside longitudinally. Caps. roundish, 5-celled, 5valved, opening at the top; valves ovate, with the margins inflexed, remote and straight; colum. subovate, terete, rugose. Seeds small, smooth, not winged.

292. CLE.THRA. Cal. 5-parted, persistent. Cor. 5petalled. Style persistent; stig. short, 3-cleft. Caps. 3-celled, 3-valver, covered by the calyx,

*** Flowers polypetalous, irregular.

993. CASSIA. Cal. 5-leaved. Pet. 5, subequal. Stam. unequal; 3 superior anth. sterile; 3 inferior. rostrate, with longer and incurved filaments. Legume membranaceous, $2 \cdot$ valred. 
294. BAPTISIA. Cal. half 4-5-cleft, bilabiate. Cor. papilionaceous; pet. nearly equal in length; vexill. with the sides reflexed. Stam. deciduous. Legume ventricose, pedicellate, many-seeded.

995. CERCIS. Cal. 5-tonthed, gibbous at the base. Cor. papilionaceous; wings larger than the vexill.; keel 2-petalled. Legume compressed, seminiferous suture margined.

\section{DIGYNIA.}

296. HYDRANGEA. Cul. superior, 5-tonthed, Pet. 5. Caps. 2-beaked, opening by a foramen between the horns.

297. SAXIFRAGA. Cal. 5-parted. Pet. 5. Caps. superior or inferior, or half inferior, 2-beaked, 2-celled, many-seeded, opening between the beaks.

998. CHRYSOSPLENIUM. Cal. superior, 4-5-cleft, coloured. Cor. 0. Caps. 2-beaked, manyseeded.

299. TIARELLA. Cal.5-parted, persistent. Pet. 5, inserted into the calyx, unguiculate. Caps. 1celled, 2-valved; valves unequal.

300. Mitella. Cal. 5-cleft, persistent. Pet. 5, pinnatifid; inserted into the calyx. raps. 1-celled, 2-valved; valves equal.

301. SAPONARIA. Cal. 1-leaved, tubular, 5-toothed, naked at the base. Pet. 5, unguiculate. Caps. oblong, 1-celled.

302. DIANTHUS. Cal. 1-leaved, tubular, 5-tonthed, with about 4 imbricate opposite scales at the base. Pet. unguiculate. Caps. cylindric, 1celled.

303. SCLERANTHUS. Cal. 1-leaved, 5-cleft. Cor. 0 . Stam. inserted into the calyx. Caps. 1-seeded, covered by the calyx.

\section{TRIGYNIA.}

304. CUCUBALUS. Cal. 1-leaved, inflated, 5-toothed. Pet. 5, unguiculate, naked at the orifice. Caps. S-celled. 
305. SILENE. Cal. 1-leaved, tubular or conic, 5-toothed. Pet. 5, unguiculate, mostly crowned at the orifice. Caps. 3-celled, 6-toothed, many-secded. 306. STELLARIA. Cal.5-leaved. Pet. 5, deeply cleft. Caps. 1-celled, opening with 6 teeth, manyseeded.

307. ARENARIA. Cal. 5-leaved. Pet. 5, undivided. Caps. 1-celled, many-seeded.

\section{PENTAGYNIA.}

308. SPERGUla. Cal. 5-leaved. Pet. 5, undivided. Caps. ovate, 5-celled, 5-valved.

309. Cerastium. Cal. 5-leaved. Pet. 5, bifid or emarginate. Caps. 1-celled, bursting at the summit with 10 teeth.

310. AGROSTEMMA. Cal. 1-leaved, tubular, coriaceous, 5-cleft. Pet. 5, unguiculate ; limb obtuse, undivided. Caps. 1-celled, opening with 5 teeth.

311. OXALIS. Cal. 5-parted, persistent. Pet. 5, slightly connected at the rlaws. Caps. pentangular, 5celled, bursting at the angles; cells 2, or manyseeded. Seeds covered with an elastic arillus.

312. PENTHORUM. Cal. 5-10-cleft. Pet. 5 or 0. Caps. 5-pointed, 5-celled; cells dividing transversely, many-seeded.

313. SEDUM. Cal. 5-cleft. Pet.5. Caps. 5, with a nectariferous scale at the base of each.

\section{DECAGYNIA.}

314. PHYTOLACCA. Cal. 5-cleft, petaloid. Bérry superior, 10-celled, 10-seeded.

\section{MONOGYNIA.}

279. ARBUTUS. Bear-berry. Ericece.

A. Uva ursi : stem procumnent; leaves cuneate-obovate, very entire, coriacenus; margin convex; flowers fasciculate ; berries 5 -seeded.

HAB. Sandy woods and mountains. Apr.-May. $\zeta$. Evergreen; $f$. rose-col.; ber. red. 
980. GAULTHERIA. Mountain-tea, \&c. Ericea.

G. procumbens: stem procumbent, with the flowering branches erect; leaves obovate, cuneate at the base, ciliate-denticulate; flowers few, terminal, nodding.

HAB. Wet or dry woods. July-Aug. h. Root creep.; leav. sempervir. $A$. woh.

981. VACCINIUM. Whortle-berry. Vaccinec.

* Leaves deciduous.

$\uparrow$ Corolla campanulate.

1. V. stamineum: leaves oval, acute, very entire, glaucous beneath; pedicels solitary, axillary, filiform; corolla spreading-campanulate; segments oblong, acute; anthers exserted, arned; berries somewhat pyriform.

HAB. Dry woods and hills. May-June. Shrub 3-4 ft. high; fl. wh.; ber. large, wh.

3. album: leaves subpubescent beneath; berries globose.

HAB. Pine barrens.

2. V. dumosum: younger branches, leaves, and racemes sprinkled with resinous atoms ; leaves obovate, cuneate at the base, mucronate, very entire, green on both sides; racemes bracteate; pedicels short, axillary, subsolitary ; corolla campanulate, segments rounded; anthers included.

HAB. Pine barrens and swamps. June. Shrub 12 -13 in. high; fl. large, woh.; ber. large, depress. black.

3. V. frondosum: leaves obovate-oblong, obtuse, very entire, and sprinkled with resinous atoms beneath, glaucous; racemes loose, bracteate; pedicels long, filiform; corolla ovate-campanulate; anthers included.

HAB. Sandy woods and swamps. July. Shrul 3-5 fl. high, ber. large, blue, pleasant. 


\section{$\dagger+$ Corolli urceolate.}

a. Flowers rucemose, or fasciculate.

4. V. resinosum: leaves petiolate, oblong-oval, mostly obtuse, very entire, sprinkled with resinous atoms beneath; racemes lateral, secund, bracteate; corolla ovate-conic, pentangular.

HAB. Woods and hills. May-June. Shrub $2 \mathrm{ft}$. high; fl.reddish; ber. black shining.

5. V. corymbosum: floriferous branches nearly leafless; leaves oblong-oval, acute at each extremity, nearly entire; the younger ones pubescent; racemes short, sessile, bracteate ; corolla cylindrical ovate.

HAB. Low grounds. May-June. Shrub $4-8 \mathrm{ft}$. high; fl. wh. or purplish; ber. black.

i. V. fuscatum: racemes somewhat naked; leaves elliptical, acute, very entire, with the nerves beneath somewhat villous; corolla cylindrical-ovate; calyx acute.

HAB. Pennsylvania. +

7. V. pennsylvanicum: branches angular, green; sessile, oval-lanceolate, mucronate, serrulate, shining on both surfaces; fascicles of Howers crowded, subterminal, corolla ovate.

HAB. Rocky hills. May-June. Shrub 12-18 in. high; bark and fl. greenish; ber. dark blue.

3. V. tcrellum: racemes bracteate, sessile; corolla ovate-cylinirical; leaves oblong-elliptic,subcuneiform, serrulate. nearly smooth.

HAB. Mountains. April.

9. V. ligustrinum: branches angular; leaves subsessile, erect, lanceolate, mucronate, serrulate ; fascicles gemmacenus, sessile; flowers nearly sessile ; corolla oblong, ovate.

HAB. Dry woods. Shrub small, slend.; leaves $\frac{3}{4}$ in. long; fl. purp.

B. Flowers solitary.

10. V. aliginosum: leaves obovate, very obtuse, en- 
tire, smooth above, veined and glaucous beneath ; flowers subsolitary, octandrous; corolla shortovate, 4-cleft ; anthers awned at the base.

HAB. High Mountains. Shrub procum. and strag. : leav. and fl. small; ber. obl.

* * Leaves sempervirent.

11. V. Vitis idcea: stem creeping; branches erect; leaves obovate, punctate beneath; nearly entire; racemes terminal, nodding; corolla oblong, campanulate.

1AB. High mountains. June. Shrub low, strag.; leav. subcoriac. rev.; fl. mostly 4-cleft, palc red; ber. red, acid.

\section{ANDROMEDA. Ericece.}

* Leaves sempervirent.

1. A. hypnoides: leaves imbricate, subulate, smooth; peduncles solitary, terminal, 1 -flowered, corolla nodding, globose campanulate.

HAB. High mountains. A small creeping shrub; $f l$. small, reh. and red.

2. A. polifolia : leaves linear lanceolate, convex; revolute, glaucous beneath; flowers in short terminal racemes.

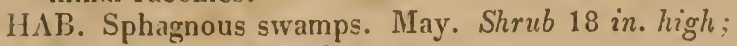
f. wh. urceol., mouth contr.

3. A. calyculata: leaves lanceolate-oblong, rather obtuse, obsoletely serrulate, ferruginous beneath; racemes terminal, leafy, subsecund; calyx bibracteate; corolla oblong-cylindrical.

IIAB. Bog-meadows. Apr.-Nay. Shrub 3-4ft. high; leaves squamulose-punct.; $f$. wh.

* Leaves deciduous.

4. A. mariana: leaves oral, somewhat acute, very entire, smooth, subcoriaceous; paler beneath; Howering branches nearly naked; pedicels fasri. 
culate; corolla ovate-cylindric; anthers simple at the summit.

HAB. Sandy woods. June-Oct. Shrub 2-4 ft.; branch. erect; fl. large, wh. or rose-col.

5. A. racemosa: leaves oval-lanceolate, acute, serrulate, membrinaceous, pubescent beneath; racemes terminal, secund, simple, (or rarely branched ;) corolla oblong-cylindrical ; anthers 4 -awned at the summit.

HAB. Swamps. June-July. Shrub 4-6 ft. high; racem. long; $A$. nod.; wh. odorous.

6. A. arborea: branches terete; leaves oblong, acuminate, acutely serrate, smooth; panicles terminal, many-spiked; corolla ovate-oblong, pubescent; anthers unawned.

HAB. Mountains. A middle sized tree; leav. shin., acid; pan. large; $f$. wh.

i. 1. ligustrina: pubescent; leaves obovate-lanceolate, acuminate, minutely serrulate, floriferous branches terminal, paniculate, naked; corolla subglobose ; anthers unawned.

HAB. Swamps. June-July. Shrub 4-8ft. high ; pan. dense; fl. small, wh.; cap. gloh.

983. KALMIA. American Laurel. Rhododeudracec.

1. K. latifolia: leaves on long petioles, scattèred and ternate, coriaceous, green on both sides ; corymbs terminal, viscidly pubescent.

HAB. Rocky hills. June-July. Shrub 4-15 ft. high; leav. semperv.; fl. large, rose-col.

2. K. angustifolia: leaves ternate, petiolate, obtuse, slightly ferruginous beneath ; corymbs linear; peduncles and caly $x$ glandular-pubescent.

HAB. Sandy woods and swamps. June-July. Shrub 2 ft. high; $f$. smaller, decper rose-col.

3. K. glauca: branches ancipitous; leaves opposite. subsessile, oblong, smooth, glaucous beneath, revolute on the margin, corymbs terminal and axillary, bracteate; peduncles and calyx very smooth.

HAB. Sphagnous swamps and mountain bogs. Tuly. Shrub 18 in. high; fl. small, rose-col. 
3. rosmarinifolia: leaves linear, conspicuously revolute. nearly green beneath.

HAB. Sphagnous sivamps. Shrub low; leav. 2 lin. broad; umbel. term.

284. RHODODENDRON. Mountain-laurel. Rhododendracece.

* Flowers pentandrous.

1. R. nudiflorum: flowers somewhat naked; leaves lanceolate-oblong, nearly snsooth, and green on both sides; the midrib bristly beneath ; margin ciliate; flowers not viscous; tube longer than the divisions; teeth of the calyx short, somewhat rounded; stamens much exseried.

HAB. Woods and copses. May. Shrub $2-6 f t$. high; fl. in terin. clust., red.

2. R. viscosum: flowers leafy ; branches hispid ; leaves oblong-obovate, acute, smooth and green on both sides, ciliate on the margin ; midrib bristly ; flowers viscous; tube as long again is the segments; teeth of the calyx short, rounded; stamens scarcely longer than the corolla.

HAB. Wet woods. June. Sirub $4-8 f t$. high; $f$. wh., viscous, srveet-scen.

ß. glaucum: leaves glaucous beneath.

3. R. calendulaceum : flowers some what naked; leaves oblong, pubescent on hoth sirles, at length hairy; flowers not viscous; teeth of the calyx oblong; tube of the corolla hairy, shorter than the segments. HAB. Pennsylvania. May. Shrub $2-6 f i$. high; fl. large, flame-col.

4. R. arborescens: flowers leafy; lcaves obovate, rather obtuse, smooth un both sides, glaucous beneath, ciliate on the margin ; nerves almost smooth, flowers not viscous; tube longer than the segments; calyx leafy, with the segments oblong. acute ; filaments exserted.

HAB. Mountains. Shrub 10-20 ft. high; $f$. lurge, rose-col.; scales of the fl.-buds yell-br., with a cil. white bord.

5. R. nitidum: flowers leafy; branches somewhat smooth; leaves oblanceolite, submucronate, co- 
riaceous, smooth on both sides, shining above, nerve bristly beneatlı; margin revolute-ciliate; flowers viscous; tube a little longer than the segments ; calyx very short ; filaments exserted.

HAB. Mountain swamps. Leav. durk green, small; fl. reddish white.

6. R. trispidum: flowers leafy; branches straight, very hispid; leaves long-lanceolate, hispid above, smooth beneath, glaucous on both sides; nerve beneath bristly; margin ciliate; flowers very viscous; tube scarcely longer than the segments; teeth of the calyx oblong, round; filaments exserted.

HAB. Mountains, near lakes. July-Aug. Shrub 10-15 ft. high, bluish, upright; $f$. wh., bord. and tube red.

** Flowers campanulate; stamens 5-10.

7. R. lapponicum: leaves elliptical, roughened with excavated punctures.

HAB. High mountains. July. A small evergreen shrub; fl. in term. clust., large purp., 4 cl.; stam. 8.

8. R. maximum : arborescent; leares oblong, acute, paler beneath; umbels terminal ; segments of the calyx oval, obtuse ; corolla campanulate.

HAB. Cedar swamps and mountain bogs. JuneJuly. Shrub 10-15 ft. high; leav. semperv.; $f$. large, rose-col.

285. RHODORA. Rhododendracect.

R. canadensis :

HAB. Mountain bogs. May. Shrub 2 ft. high; leav. oval; fl. in term. umb., purplish; stam. not cxsert.

286. EPIG $\mathrm{AA}$. Ground-laurel. Ericece.

E. repens: branches, nerves of the leaves, and petioles, very hairy; leaves cordate-ovate, very entire ; corolla subcylindrical.

HAB. Rocky woods. April. 4. A trailing evergreen; fl. clust., reddish wh. 
287. PTEROSPORA. Monotropece.

$P$. andromedea.

HAB. Steril hill-sides. July-Aug. 4. ? Stem $1 \frac{1}{2}-$ $2 \mathrm{ft}$. high, simple, scaly, brownish; leaves $0 ; f$. in a long raceme, nutant, reddish rohite.

288. MONOTROPA. Bird's-nest. Monotropec.

* Scapes many-flowered. Нy popithys.

1. M. lanuginos $\alpha$ : scape bearing the flowers in a spike; bracts and flowers woolly.

HAB. Beech woods. Aug. 4.? Scape scaly, aggreg., 4-6 in. long; $f$. secund, yellowish, parasitic on roots.

2. Hypopithys: scape bearing the flowers in a spike; scales and flowers smooth externally ; lateral flowers octandrous.

HAB. Beech woods. July. 4.? Plant dingy yellow. Parasitic. + .

\section{* Scape 1-flowered.}

3. M. unifiora: scape straight, elongated, 1-flowered; flowers decandrous, erect or cernuous.

HAB. Woods. June. Root roundish; plant white: scape 5-8 in. high; fl. large.

989. PYROLA. Winter-green. Monotropece.

* Flowers racemed, pointing in various directions.

1. P. rotundifolia: leaves rounded or dilated oval, obsoletely serrulate, subcoriaceous and shining; petiole about as long as the lamina; scape many-flowered; style declinate.

HAB. Dry woors. July-Aug. 4. Leav. rad. semperv.; fl. large, reddish white.

$\therefore$ P. elliptica: leaves membranaceous, elliptical-ovate; serrulate, rather acute; lamina longer than the petiole; scape nearly naked; bracts subulate; calyx 5 -toothed; style declinate.

HAB. Dry woods. July-Aug. 4. Scape 10 in.

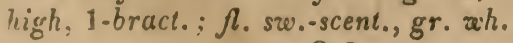

Q 2 
3. P. asarifolia: leaves subreniform, generally emar ginate, coriaceous; lamina shorter than the petiole: scape mostly convolute, many-flowered; calyx appressed; stamens slightly ascending; style declinate, clavate.

HAB. Sandy woods. July. 4. Leav. small, dark gr.; scape 8-10 in. high; bract solit.; $f$. greenish, inodor.

4. P. minor: leaves roundish-oval, serrulate; scape naked; style short, straight, as long as the erect stamens.

HAB. New-York, \&c. June. 24. Fl. small, reddish wh.; stig. pelt.

** Flowers racemed, secund.

5. P. secunda: leaves roundish-ovate, acute, serrate; racemes secund; styles straight.

HAB. Sandy woods. June-July. 24. Plant a span high; stem assurg.; $f . g r$. reh.

*** Flower solitary, terminal.

6. P. uniflora: leaves orbicular, serrate; scape 1 flowered; style straight.

HAB. Dry woods and sphagnous swamps. July. if Fl. large, red. wh.

**** Flowers somezhat umbellate; styles very short.

Chimaphila.

\%. P. umbellata: leaves cuneate-lanceolate, acute at the base, serrate, uniformly green, scape corymber. HAB. Dry woods. July. 4. Stem uscend.; leav. coriac., semperv.; fl.purpl. white.

8. P. maculata: leaves lanceolate, rounded at the base, remotely serrate, discoloured; scape $2-3$-flowered.

HAB. Dry woods. July-Aug. 24. Evergreen.: leav. varieg.; fl. purp. wh., nod.

290. LEDUM. Labrador tea. Rhododendracea.

1. L. latifolium: leaves oblong, replicate on the mar: 
gin, ferruginous-tomentose beneath ; stamens 5, along as the corolla.

HAB. Sphagnous sivamps. June. 4. A small evergrcen shrub; $f$. corymb., wh.

2. L. palustre: leaves linear, revolute on the margin ; ferruginous-tomentose beneath ; stamens 10 , longer than the corolla.

HAB. Borders of mountain lakes.

\section{LEIOPHYLLUM. Rhododendracece.}

L. buxifolium.

HAB. Pine barrens and high mountains. May. $A$ small evergreen shrub, erect; leav. oval, half in. long: fl. num. in term. umb., wh.

\section{CLETHRA. Ericece.}

C. alnifolia : leaves cuneate-obovate, acute, coarsely serrate, smooth and green on both sides; racemes spiked, simple, bracteate, hoary-tomentose.

IIAB. Swamps. Aug. Shrub 4-8 ft. high; lear. alt.; $f$. woh., sweet-scent.

\section{CASSIA. Wild-pea. Leguminosa.}

1. C. marilandica: nearly smooth; leaves in 8 pairs. lanceolate-oblong, mucronate; gland on the petiole obovate ; racemes axillary, many-flowered; legume linear, arcuate.

HAB. Wet meadors. Aug. 24. Stein $\approx-4 \mathrm{ft}$. high; $\lambda$. yellow, in large axill. racem.

2. C. Chamcecrista: smoothish; leaves in many pairs, linear, with the gland on the petiole subpedicellate; buds 2-3-flowered; 2 of the petals spotted; legume pubescent.

HAB. Sandy fields. June-May. ๑. I foot high; leav. somezhat sensitive; $\mu$. yellow, in bract. jas cic.; base of 3 pet. spotted.

3. C. niclitans: steun spreading, pubescent; leaves in many pairs, linear; gland on the petiole pedicellate; peduncles fasciculate, few-flowered ; llowers pentandrous; stamens equal. 
HAB. Sandy fields. Aug. ๑. A foot high, slend.; fl. viery small, axill.

4. C. fasciculata: nearly smooth; leaves at the summit in 9 pairs; gland sessile near the middle of the petiole; fascicles lateral, many-flowered; petals and stamens of the same colour; legume smooth. HAB. Dry fields.' June-Aug. $\odot$. Fl. smaller than No. 2 ; leg. curved.

294. BAPTISIA. Wild Indigo. Leguminosce.

B. tinctoria: very smooth, much branched; leares ternate, subsessile; leaflets rounded-obovate; racemes terminal, (flowers yellow ;) legume on il long stipe.

HAB. Sandy woods. July.-Sept. 4. Stern 2$3 \mathrm{ft}$. high, much branch. Dries black.

295. CERCIS. Judas' tree. Leguminosce.

C. canadensis: leaves roundish-cordate, acuminate. villous at the axils of the nerves; legumes on short footstalks.

HAB. Woods. April. A small tree; leav. alt., large : fl. red, in small lat. fascic., pracoc.; leg. compress.

\section{DIGYNIA.}

296. HYDRANGEA. Saxifragece.

1. H. vulgaris: leaves oblong-ovate, smooth beneatb, obtuse at the base, acuminate, dentate; cymes naked.

HAB. Shady rocks. July. Shrub 3-6 ft. high : leav. oppos.; $f$. wh.

2. H. radiata: leaves ovate, acuminate, serrate, tomentose and white beneath; cymes radiate.

HAB. Pennsylvania. July. Shrub $6 \mathrm{ft}$. high; ext. fl. of the cymes large, rch. neut.

29\%. SAXIFRAGA. Saxifrage. Saxifragece.

$\therefore$ S. virginica: pubescent; leaves oval, obtuse, crenate, narrowed at the base into a petiole; stem nearly leafless, corymbose-paniculate ; flowers sub- 
sessile; petals oval, with branched nerves; capsule half inferior.

HAB. Rocks and hills. April-May. 4. Stem: succul., 3-12 in. high; leav. thick; fl. zoh.

2. S. pennsylvanica: pubescent, leaves oblong-lanceolate, acute at each extremity, obsoletely denticu-

- late; stem naked; panicle oblong; flowers fasciculate; petals linear, longer than the calyx ; capsule superior.

HAB. Swamps. May-June. 4. Leav. rad., 58 in. long; scape $1 \frac{1}{2}-2 \mathrm{ft}$. high; pet. yell. green.

298. CHRYSOSPLENIUM. Golden Saxifrage. Saxifragece.

C. oppositifolinum: leaves opposite, roundish-cordate. HAB. Wet places. April-Mlay. H. Pl. succul., yell. gr., dichot.; fl. sess., 8-an.

\section{TIARELLA. Saxifragece.}

T. cordifolia: leaves cordate, acutely lobed, dentate ; teeth mucronate; scape racemed.

HAB. Rocky hills. May. 4. Leav. rad., pubes.; fl. in a simp. racein., woh.

300. MITELLA. False Sanicle. Saxifragece.

1. M. diphylla: leaves somewhat lobed, with the lobes acute and dentate; stem erect, with two opposite leaves above the mildle.

HAB. Banks of creeks. May. 4. A foot high, simp. slend.; racem. simp.; fl. wah.

2. M. cordifolin: leaves orbicular-reniform, doublycrenate, with scattered hairs above; scape erect or prostrate, naked, or with a single leaf.

HAB. Moist rocks. June. 4. Stem. slend. raith. creeping stolons; $f$. few', wh.

301. SAPONARIA. Soap-wort. Caryophyllece.

S. officinalis : leaves ovate-lanceolate; calyx cylindric, smooth.

HAB. Waste places, \&c. June-Sept. H. Stem $18 \mathrm{in.}$ high, scab.; fl. large, rosc-col. 
302. DIANTHUS. Pink. Caryophyllece.

D. Armeria: flowers aggregate; fasciculate; scales of the calyx lanceolate, villous as long as the tube. HAB. Sandy soils. July. ○. A foot high, pubes.; leav. lin. lan.; fl. red, punct. \$?

302. SCLERANTHUS. Knawel. Caryophyllex.

S. annurs: calyx of the fruit spreading, acute ; stem spreading.

HAB. Sandy fielis. July. ○. Stem num.; pro-

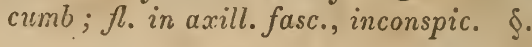

\section{TRIGYNLA.}

304. CUCUBALUS. Campion. Caryophollece.

1. C. Behen: smooth and glaucous, decumbent; leaves oblong-oval, acute, nerveless; calyx membranaceous, with reticulated veins.

HAB. Rocky hills. July. 4. Stem $18 \mathrm{in.} \mathrm{high,}$ panic.; fl. large, nod.; pet. roh.

2. C. niveus: stem livaricate and dichotomous above; leaves oblong-linceolate, minutely and pulverulently pubescent ; the uppermost ovate ; calyx obtuse, campanulate, inflated, subpilose; petals small, reflexed, bifid at the extremity; claws exserted beyond the calyx, nearly naked; flowers solitary, dichotomal, terminal.

HAB. On an island in the Susquehanna. Fl. remote, solit.; pet. wh.

3. C. stellatus: erect, pubescent; leaves verticillate, in fours, oval-lanceolate, long-acuminate.

HAB. Woods. July-Aug. 4. Stcm 2-3 ft. high; pan. term.; $f$. wh.; cal. not reticul.

305. SILENE. Catch-fly. Caryophyllece.

1. S. pennsylvanica : viscidly-pubescent; radical leaves cuneate; stem leaves lancerlate ; panicles trichotomous ; petals slightly emarginate, very obtuse, subcrenate.

HAB. Rocks and sandy woods. Mav-June. 4. 
Stems num., 8-12 in. high; fl. in term. trichot. panic., purp.

2. S. virginica: erect or decumbent, viscidly pubescent; leaves oblong-lanceolate, scabrous on the margin; panicle dichotomous; petals bifid; stamens exserted.

HAB. Pennsylvania. June. H. A foot high, mostly simp. ; fl. fer , crimson.

3. S. antirrhina: leaves lanceolate, minutely ciliate; panicles trichotomous; calyx ovate; petals small, bifid, stamens included.

HAB. Hills and rocky banks. June. ๑. Stem 1 -2ft. high, slender; fl. small; pet. wh.

1. S. nocturna: flowers spiked, alternate, sessile, secund ; petals bifid.

HAB. Pennsylvania. ๑. Fl. scss., obov.; pet. $\approx h$.. bifid, rather large.

306. STELLARIA. Stitchwort. Caryophyllece.

1. S. pubera: pubescent; stem decumbent; leaves ovate-oblong, sessile, acute, subciliate, somewhat undulate, pedicels dichotomal, recurved; petals longer than the calyx.

IIAB. Shady rocks. May-June. 4. Stem 6-$10 \mathrm{in.}$ long; fl. large, weh.

$\therefore$ S. media: stem procumbent, with an alternate, pubescent, lateral line; leaves ovate, smooth; petals 2-parted ; stamens 5--10.

HAB. Cultivated grounds, \&c. ๑. Stem zeeak; leav. petiol.; pedun. 1- $f$.

3. S. longifolia : very smooth ; stem erect, quadrangular, weak ; leaves linear-lanceolate ; panicle terminal ; peduncles divaricate, very long, bracteate; petals broad-obovate, 2-parted, longer than the acute 3-nerved calyx; styles 3-4.

IIAB. Wet places. June. 4. Stem $1 \mathrm{ft}$. high, dichot., leav. $1 \frac{1}{2}-2$ in. long; pan. loosc.

1. P. lanceolata : very smooth, procumbent or ascending; leaves lanceolate, acute at each end; petals about as long as the calyx ; stigmas mostly 4 , or wanting.

HIB. Mountain bogs. June. It. Stem diff., 6- 
3 in. long, $f$. axill. and term., sometimes apel.: stam. 8-10.

\section{ARENARIA. Sandwort. Caryophyllece.}

* Leaves without stipules at the base.

1. A. peploides: stem dichotomous; leaves ovate, acute, fleshy; calyx obtuse.

HAl3. Sea-coast. July. 4. Stem decumb.; 8-12 in. long, thick; fl. subsol. axill.

1. A. lateriflora: stem filiform, simple or branched, pubescent; leaves ovate, subtriply nerved; peduncle lateral, solitary, elongated, bifid; one of the pedicels with 2 opposite bracts near the middle; corolla longer than the calyx.

HAB. Bog meadows. June. 4. Stem 5-8 in. high; leav. sess., pale gr., punct.

3. A. serpyllifolia: stem dichotomous, diffuse; leaves ovate, acute, subciliate; calyx hairy; exterior leaflets 5-ribbed.

HAB. Sandy fields. May-JuIy. ○. Stem 3-8 in. high; leav. minute, approx.

1. A. squarrosa: cespitose ; inferior leaves squarroseimbricate, canaliculate pungent ; stem simple, fewleaved ; flowers few, terminal, erect ; petals much longer than the roundish calyx.

HAB. Pine barrens. May-Aug. 4. Stem num.. $6 \mathrm{in}$. long; leav. crowed.; $f$. large.

\%. A. glabra: very smooth; stems numerous, erect, filiform; leaves subulate-linear, flat, spreading; pedicels 1 -flowered, elongated, divaricate; leaflets of the calyx oval, obtuse, smooth, shorter than the petals.

HAB. Mountains. June. 4. Stem 4-6 in. high; leav. remote; ped. trichot.

i. 1. stricta: smooth; stems numerous, filiform, simple; leaves subulate-linear, subfasciculate, spreading; leaflets of the calyx ovate, acute, conspicuously 3-nerved, half as long as the petals.

HAB. Mountains. May-June. 4. Sterns 6-19 in. high; leav rather rigid. 
* Leaves with stipules at the base.

\%. A. rubra: stems prostrate; leaves narrow-linea:, acute, flat, somewhat fleshy, mucronate; stipules: sheathing, ovate, cleft ; capsule as long as the calyx; seeds compressed, angular, roundish.

HAB. Sandy fields. ๑. Sinooth, spreading; cal viscid-pubes.; fl. red; stam 5-10.

3. marina: leaves cylindrical, fleshy, unarmed.

HAB. Salt marshes. May-Nov. $\odot \hat{\mathcal{E}}$. Stem smooth; $f$. dark rose-col.; seed compr.

\section{PENTAGYNIA.}

308. SPERGULA. Spurrey. Caryophyllec.

i. S. arvensis: leaves verticillate; panicle dichoto mous ; peduncles of the fruit reflexed.

HAB. Sandy fields. June-Aug. ๑. Stom $6-10$ in. high; leav. fliff., 8-10 whorled. \$.

2. S. sasinoides: glabrous; leaves opposite, subulate, awnless ; perluncles solitary, very long, smooth.

HAB. Sandy fields. July. ○. Stem spread., :3 in. long; ped. axill. and term.

309. CERASTIUM. Nouse-ear Chickweed. Caryo. phyllece.

* Petals not exceeding the calyx in length.

1. C. vulgatum: hairy and viscid, cespitose, suberect, leaves ovate; flowers capitate, longer than their pedicels.

HAB. Ficlds and dry hills. May-Sept. ๑. Plant pale green, viscid when young. §.

2. C. viscosum: hairy and viscid, spreading; leaver oblong-lanceolate; florvers somewhat panicled, shorter than their pedicels.

HAB. Ficlis and road sides. May-Sept. 4. Darker green, spreading, rarely visc.

3. C. semidecandrum: hairy and viscid, suberect; leaves oblong-ovate; flowers somewhat panicled R. 
shorter than their panicles; stamens 5 ; petals slightly notched.

HAB. Dry hills. ○. Stems num., assurg. ; pet. often shorter than cal.

4. C. hirsutum: very hairy, diffuse; leaves obtuse, connate ; flowers clustered; petals 2-cleft, a litlle longer than the acute calyx.

HAB. New-York, \&c. $\odot$. Leav. subovate, half in. long ; $f$. in dense clust.

* Petals longer than the calyx.

5. C. nutans: viscid and elongated; stems erect, straight, deeply striate; leaves elongated, distant,

- linear-oblong, acute; petals oblong, bifid at the tip, longer than the calyx; peduncles at length much longer than the flowers.

HAB. Rocky hills. June. ○., Pale green, 8-12 in. high, very visc.; pan. loose, term.

6. C. oblongifolium: cespitose, pubescent; stem erect, terete, even; leaves lanceolate-oblong, rather acute, shorter than the joints; tlowers terminal. shorter than the pedicels; petals obovate, bifid at the tip ; twice the length of the calyx.

HAB. Mountains. June. 24. Stem 8 in. ligh, retrors. pubes.; ped. bract., fl. large.

\%. C. tenuifolium: pubescent, cespitose; leaves nar. row-linear, longer than the internodes; stems short, 3-flowered at the summit; petals obovate, emarginate, 3 limes as long as the calyx.

HAB. Mountains. June. 4 . A span high; leav. long, 1 line broad; pedunc. elong.

310. AGROSTEMMA. Corn-cockle. Caryophyllea.

A. Githago: hairy; calyx much longer than the corolla; petals entire, without a crown.

HAB. Corn-fields. June-July. ๑. Plant paie green, 2 fi. high; fl. large, purp.

311. OXALIS. Wood-sorrel. Oxalidece.

* Stemless.

1. O. Acctosella : stemless; scape 1.flowered, longes 
than the leaves; leaves ternate, dilated-obcordate, pilose; styles as long as the interior stamens; root rentate.

HAB. Mountains. June. 4. Leav. often purple beneath; fl. large, woh. veinerd.

2. O. violacea : stemless; scape umbelliferous; flowers nodding; leaves ternate, obcordate, sinooth ; segments of the calyx callous at the tip ; styles shorter than the exterior stamens ; root squamose.

HAB. Rocky woods. April-June. H. Root thick, with ciliate scales; $f$. violet.

\section{** Caulescent.}

3. O. corniculata: pubescent; stem prostrate; leaves ternate, obcordate; umbels as long as the petioles; petals obovate; slightly emarginate ; styles as long as the interior stamens.

HAB. Cultivated grounds, \&c. May-Sept. $\odot$. Stem 6-8 in. long; $f l$. sm., yell.

1. O. stricta: hairy; stem erect, branched; leaves ternate, obcordate; umbels longer than the petioles; petals obovate, entire; styles as long as the interior stamens.

HAB. Sandy fields. May-Aug. 4.? Stem 4$10 \mathrm{in.} \mathrm{high,} \mathrm{never} \mathrm{creeping;} \mathrm{fl.} \mathrm{sm.,} \mathrm{yell.}$

312. PENTHORUM. Virginia Stone-crop. Semperviver.

P. sedoides: stem branched, angular above; leaves lanceolite, subsessile, unequally serrate; spikes simple, secund, recurved, paniculate; seeds elliptical.

HAB. Wet places. July-Aug. 4. Stein $12-18$ in. high; leav. alt.; pet. mostly 0.

313. SEDUM. Stone-crop. Semperrive.

1. S ternatum : creeping; leaves flat, rounded-spathulate, ternate; cymes mostly 3-spiked; terminal flowers decandrous; the rest octandrous.

$\mathrm{HAB}$. On rocks. May. If Stem $1-6 \mathrm{in}$. long : Leav. entire; $\Omega$. wh.; unth. darl. 
2. telephioides: leaves broad, flat, ovate, acute at each end; corymbs many-flowered.

HAB. Rocky hills. «. A foot high, leafy, purplish; fl. pale purp.

\section{DECAGYNIA.}

314. PHYTOLACCA. Poke. Atriplices.

P. decandra: leaves ovate, acute at each end; flowers with 10 stamens and 10 styles.

HAB. Waste places. June-Oct. 4. Smooth. 4-8 ft. high. purp.; fl. racem. wh.; ber. purp.

\section{ICOSANDRIA.}

\section{MONOGYNIA.}

315. CACTUS. Cal. superior, many-cleft; segments imbricate. Pet. numerous, inserted in several series; the interior ones larger. Stig. manycleft. Berry 1-celled, many-seerled.

316. PRUNUS. Cal. inferior, campanulate, 5-cleft, deciduous. Pet. 5. Drupe even. Nut with a prominent suture.

317. LY'THRUM. Cal. tubular-campanulate, 6-12toothed. Pet. 6, equal, inserted upon the calyx. Caps. 2-4-celled, many-seeded.

31. CUPHEA. Cal. ventricose, tubular, 6-12-toothed, unequal. Pet. 6, unequal, inserted into the calyx. Cups. with the calyx bursting longitudinally, 1 -celled.

\section{DI-PENTAGYNIA.}

319. AGRIMONIA. Cal. inferior, 5-cleft, with a lobed calicle at its base. Pet. 5. Stam. 12. Achen. 2 , in the bottom of the calyx.

390. CRAT 1 AGUS. Cal. superior, 5-cleft. Pet. 5. Styles 1-5. Fruit pulpy, closed, with from $2-$ 51 -seeded nuts.

321. SORBUS. Cal. 5-cleft. Pet. 5. Styles 2 or 3. Berry inferior, farinaccous, with 3 cartilaginous seeds. 
392. SESUVIUM. Cal. 5-parted, coloured. Pet. 0. Caps. superior, ovate, 3-celled, opening transversely all round, many-seeded.

323. ARONIA. Cal. 5-toother. Pet. 5. Berry inferior, 5-10-celled; cells 1-2-seeded. Seeds cartilaginous.

324. PYRUS. Cal. 5-cleft. Pet. 5. Apple inferior, large and fleshy, 5-celled, many-seeded. Seeds cartilaginous.

225. SPIRÆA. Cal. inferior, 5-cleft, expanding. Pet. 5 , equal, roundish. Stum. numerous, exserted. Caps. numerous, (3-12.) 1-celled, 2-vaived. each 1-3.3 seeded.

226. GILLENIA. Cal. tubular-campanulate; border 5-toothed. Cor. partly unequal. Pet. 5, lanceolate, attenuate at the base. Stam. few, included. Styles 5. Caps. 5, connate at the base, opening on the inner side, each 2 -seeled.

\section{POLYGYNIA.}

327. ROSA. Cal. urceolate, fleshy, contracted at the orifice; border 5 -cleft. Pet. 5. Seeds numerous, hispid, attached to the interior side of the calyx.

328. RUBUS. Cal. 5-cleft, inferior. Pet. 5. Fruit composed of many 1 -seeded jricy acines, on a dry receptacle.

329. DALIBARDA. Cal. inferior, 8-cleft, spreading. Styles 5-8, long and deciduous. Secds dry.

330. DRYAS. Cal. 8-10-cleft, segments equal. Seeds $5-8$, with long plumose awns.

331. GEUMI. Cal. 10-cleft, inferior; the alternate segments smaller. Pet. 5. Seeds awned, with the a zan naked or bearded, mostly geniculate.

332. POTEN'TILLA. Cal. 1lat, 10-cleft, (rarely 8 or 12-cleft ;) segments alternately smaller. Pet. 5 , (rarely 4.) Seeds or acines subovate; moslly rugnse, immerged in a common receptacle, which is juiceless or spongy, more or less hemispherical.

333. Fragralild. Cul. 10-cleft. Pct, 5. Acines $\mathrm{R} \approx$ 
naked, fixed on a large, pulpy, deciduous receptacle.

334. CALYCANTHUS. Cal. urceolate, many-cleft above; segments squarrose, coloured, petaloid. Cor. 0. Styles numerous. Seeds numerous, naked. smooth, included in a ventricose, succulent caly $x$.

\section{ICOSA NDRIA.}

\section{MONOGYNIA.}

315. CACTUS. Indian Fig, \&c. Cacti.

C. Opuntia: articulately proliferous; articulationa compressed, ovate; spines setaceous ; fruit succulent, smooth.

HAB. Rocks and Sandy fields. June-July. 4. Procumb., leafl., fleshy; fl. yell.

316. PRUNUS. Plum and Cherry. Rosacea.

* Fiozerers racemose.

1. P. virginiana: racemes erect, elongated; leaves deciduous, oral-oblong, acuminate, shining above, serrate, smooth on both sides; petioles with $2-1$ glands.

HAB. Woods. May. A large tree; racem. simp.: fl. rh. ; drupe black.

2. P. serotina: racemes loose, at length pendulous; leaves deciduous, ovate, with a short acumination, doubly and very acutely serrate; millrib bearded on each side towards the base; petiole with: glands.

HAB. Woods. May. A small tree; leav. thin: drupe red.

3. P. canadensis: flowers in racemes; leaves decidu. ous, without glands, broad-lancenlate, rugose, pubescent on both sides.

HAB. Pennsylvania. Branch smooth; leuv. withoul glands; base atten. +.

* Peduncles subumbellate or solitary.

4. P. pennsylvanica: flowers subcorymbed; pedicels 
elongated ; leaves ovate-oblong, acuminate, erosely denticulate, smooth; petioles with 2 glands; branches punctate; fruit subovate.

HAB. Mountains. June. A small tree; leav. memb.; corymb. 6-8-fl.; drupe red.

5. P. nigra: umbels sessile, solitary, few-flowered; leaves deciduous, ovate, acuminate, unequally and acutely serrate; smooth on both sides; petioles with 2 glands.

HAB. Mountains. June. Shrub 6-8 ft. high; branch. red; ped., smooth, short.

6. P. pygmcea: umbels sessile, few-flowered ; leaves ovate, acute, smooth on each side, acutely serrate, with 2 glands at the base.

HAB. New-England, \&c. Shrub 3-4 fl. high; fruit small, black, indifferent.

7. P. pubescens: leaves sessile, aggregated, few-flowered; peduncles and calyx pubescent ; leaves shortoval, serrulate ; drupe spherical.

HAB. Sandy shores and hill sides. May. Slirub 23 ft. high; fruit br. purp., glauc.

3. P. pumila: umbels aggregated, sessile, few-flowered; calyx acute; branches virgate, terete; leaves narrow-lanceolate, serrate above, pale beneath.

HAB. Banks of rivers. May. Shrub 2-3 ft. high ; leav. long; fruit red, acid.

7. P. depressa: umbels sessile, aggregated, few-flow. ered; calyx obtuse; branches angular, depressedprostrate ; leaves cuneate-lanceolite, remotely serrate, smooth, glaucous beneath.

HAB. Sandy shores. A shrub, low, spreading; fr. sinall, black, agreeable.

10. P. maritima: peduncles subsolitary ; leaves ovateoblong, acumiuate, duubly serrate.

HAB. Sea-coast. Fruit large, dark purp.

19. P. mollis: younger branches, leaves and peduncles pubescent; umbels sessile, $2-3$-llowered ; leaves ovate, long-acuminate, doubly dentate-serrate: stipules setaceous, denticulate; calyx nearly smooth ; segments linear-lanceolate, serrate.

HAB. Woods. A small tree; leav. 2-3 in. long ; $f r$ oval, large, nearly black. 
317. LYTHRUM. Loose-strife. Salicaria.

* Calyx subcampanulate, 10-toothed; capsule 3-4celled. Decodon.

1. L. verticillatum: pubescent; leaves opposite and ternate, lanceolate, petiolate ; flowers axillary, verticillate, decandrous; petals undulate; fruit subglobose.

HAB. Swamps. Aug. 4. Stem $2 \mathrm{ft}$. high, 6ang. ; $f$. in axill. corymbs, purp.

* Calyx tubular, capsule 2-celled.

2. L. Salicaria. $\beta$. pubescens: pubescent; leaves opposite and ternate, sesstle, lanceolate, cordate at the base ; flowers with 12 stainens, terminal, verticillate-spiked; capsule oblong.

HAB. Wet meadows. 24. About $2 \mathrm{ft}$. high; $\mathrm{fl}$. purp., very showy. +..

3. L. hyssopifolium: leaves alternate and opposite, linear-lanceolate and subeliiptic ; flowers axillary. hexandrous.

HAl3. New-York. 24. Stem mostly simp., quadrang., submargined; fl. pale purp.

\section{CUPHEA. Salicarice.}

C. viscosissima: viscous; leaves opposite, petiolate, ovate-oblong; flowers lateral, solitary, on short peduncles; stamens 12.

HAB. Fields and mountains. Sept. ๑. Stem 1218 in. high, pubes.; fl. purp.

\section{DI-PENTAGYNIA.}

319. AGRIMONIA. Agrimony. Rosacece.

1. A. Eupatoria: hairy; cauline leaves interruptedly pinnate; leaflets ovate, with the terminal one petiolate, acutely dentate, smoothish; spike virgate; petals twice as long as the calyx; fruit turbinate, hispid, smooth at the base. 
HAB. Woods and hedges. June-Aug. \&. Stem 2 fi. high, sirrip.; fl. small, yellow.

3. hirsuta: whole plant very hairy.

2. A. parviflora: hairy ; leaves interruptedly pinnate, with the terminal one sessile; leaflets numerous, mostly linear-lanceolate, incisely serrate; spike virgate; flowers on very short pedicels; petals one and a half the length of the calyx ; fruit roundish, divaricately hispid.

HAB. Woods. Aug. 4. Fl. small, yellore.

320. CRATAEGUS. Hawthorn. Rosacece.

1. C. coccinea: thorny ; leaves on long petioles, orate, subcordate, acutely lobed and serrate, petioles and the pubescent calyx glandular; flowers pentagynous.

HAB. Woods. May. A large shrub; spines long; fl. corymb., wh. ; fr. red, edible.

2. C. cordata: thorny; leaves cordate-ovate, pinnatifid, lobed and angled. smooth ; petioles and calyx without glands; flowers pentagynous.

HAB. Hedge rows and river hanks. A large shrub; corymbs. comp. ; fr. red, glob.

3. C. pyrifolia: thorny or unarmed; leaves ovateelliptic, incisely serrate, somewhat plicate and hairy; calyx villous; segments linear-lanceolate, serrate, flowers trigynous.

HAB. Rocky woods, \&c. June. $\Upsilon_{2}$. Leav. large, sublob.; pedunc. and cal. toment

4. C. elliptica: thorny; leaves elliptical, unequally serrate, smooth; petioles and calyx glandular; fruit globose, 5-seeded.

HAB. Copses and dry swamps.

c. C. glandulosum: thoruy; leaves obovate-cuneiform, smonth and shining; petioles, stipules and calyx glandular ; fruit oval. 5 -seeded.

IJAB. Mountains. May. $T_{2}$. Fruit middle-sized, scarlet.

b. C. purvifulia: thorny ; leaves ohovate, subsessile. deeply and obtusely serrate, subtomentose; leaflets of the calyx laciniate; flower's subsolitary, pentagynous. 
HAB. Sandy woods. June. Shrub $4 \mathrm{ft}$. high: branch. toment.; fr. large, yellow.

7. C. punctata: thorny or unarmed; leaves obuvatecuneate, subplicate, smooth, incisely serrate, decurrent at the base into a petiole; calyx villous ; segments subulate, very entire; berries subglobose, with the summit depressed.

HAB. Woods and swamps. May. A small tree; vcins of the leav. hairy; fr. yellow or red.

3. C. Crus galli: thorny; leaves obovate-cuneiform, subsessile, shining, coriaceous ; corymbs compound; segments of the calyx lanceolate, serrate ; llowers digynous.

HAB. Woods, \&c. June. A small tree, with long spines; style often solit.; fr. red.

\section{SORBUS. Service-tree. Rosacece.}

1. S. americana: leaves pinnate; leaves somewhat equally serrate, and with the common petiole very smooth.

HAB Mountains. MIay. A large shrub; leafl. pairs; corymbs term. ; fr. fulv.

2. S. microcarpa: leaves pinnate; leaflets acuminate, acutely and incisely serrate, and with the common petiole smooth; serratures setaceously mucronate. HAB. High mountains. A large shrub; young branch. glossy; fr. sm. scarlet.

\section{SESUVIUM. Ficoidece.}

S. sessile: flowers sessile; leaves linear-oblong, flat. HAB. Sea-coast. Succul.; leav. oppos.; $f$. axill.. solit., rosaceous.

\section{ARONIA. Rosacece.}

1. A. arbutifolia: unarmed; leaves ovate-oblong, acute, crenulately serrulate, tomentose beneath: flowers in corymbs; calyx tomentose.

HAB. Low thickets. May. Shrub o-4 ft. high: fruit scarlet, sreetish and astring.

5. inelanocarpa: leaves beneath, and the calyx emooth; fruit black. 
HAB. Mountains; rarely in bogs. Leav. narrower; fruit black.

z. A. Botryapium: unarmed; leaves cordate, oval, conspicuously acuminate, glabrous when mature; flowers in racemes; petals linear-lanceolate.

HAB. Low woods. May. A small tree; young leav. silky; fr. purp., eatable.

3. A. ovalis: unarmed; leaves roundish-elliptical, acute, smooth; flowers in racemes; petals obovate; germens and segments of the calyx pubescent.

HAB. Swamps. A small shrub; ber. black and eat= able.

324. PYRUS. Apple and Pear. Rosacece.

8. P. coronaria: leaves broadly-oval, round at the base, somewhat angular, serrate, smooth; pedun. cles racemose.

HAB. Woods. May. A small tree; fl. large, frag., fr. yell., depress., acid.

?. P. angustifolia: leaves lanceolate-oblong, acute at the base, slightly crenate-dentate, shining; peduncles corymbose.

IIAB. Pennsylvanial. May. A small tree; lear. and fr. smaller than $\mathcal{N}$ o. 1 .

325. SPIRÆA. Meadow-sweet. Rosaceæ.

* Frutescent.

1. S. salicifolia: leaves lanceolate, acutely serrate, smooth; racemes terminal, compound, panicled; flowers pentagynous.

HAB. Wet meadows. July. Stem 2-4 ft. high; leav. obluse or acute; fl. whh.

3. S. tomentosu: leaves ovite-lanceolate, unequally serrate, tomentose beneath; racemes terminal, compound, crowded; flowers pentagynous.

HAB. Low grounds. July $-A$ ug. Suffrut. $2-3 f t$. high ; racem. elong. ; $A$. pærp.

3. S. hypericifolia: leaves obovate, very entire, or dentate at the summit, smooth ; umbels sessile.

IIAB. Dry swamps. May. Shrub $3 \mathrm{ft}$. high; branch。 slend; fl. num., wh. 
4. S. corymbosa: lenves oblong-obovate, incisely-den tate; pubescent beneath ; corymb terminal, pedunculate, compound, fastigiate, sumewhat leafy : flowers pentagynous.

HAB. Mountains. Suffrut. $18 \mathrm{in.} \mathrm{high;} \mathrm{corymb.}$ many-fl., wh. or rose-col.

3. S. crenuta: leaves obovate, acute, toothed at the summit, 3-nerved; corymbs crowded, pedunculate. HAB. New-York. +.

6. S. opulifolia: leaves ovate, lobed, douhly toothed and crenate, smooth ; corymbs terminal, with the flowers crowded and trigynous; capsules inflated. HAB. Rocky banks. June-July. Shrub 3-5 ft. high; corymb simp.; $f$. zeh.

\section{* Herbaceous.}

*. S. aruncus. B. americana: leaves twice or thrice pinnate, shining; spikes slender, in an oblong panicle; flowers trigynous, perfect.

HAB. Mountains. June. 4. Stem 4-6 ft. high ; fl. wh.

8. S. lobata: leaves pinnate, smooth; terminal leaflet much larger and 7-lobed; lateral leaflets 3-lobed: corymbs proliferous.

HAB. Pennsylvania. June. 4. Leafl. subpalm., lobed; fl. rose-col., 3-5-gyn.

\section{GILLENIA. Rosacece.}

1. G. trifoliata: leaves ternate, lanceolate, serrate; stipules linear, entire; flowers in terminal loose panicles.

HAB. Rocky hills. June. 4. Stem $2 \mathrm{ft}$. high, red; fl. large, wh. ; pet. elong.

2. G. stipulacea: radical leaves pinnatifid; stem leaves ternate; leaflets incisely-serrate; stipules foliaceons, ovate, incisely dentate; flowers in loose terminal panicles.

HAB. Mountains. i4. Stem $2-3 \mathrm{ft}$. high, red, stip. very large; $f$. wh. 


\section{POLYGYNIA.}

\section{ROSA. Rose. Rosacece.}

^. R. parviflora: fruit depressed-globose, and with the peduncles hispid; petioles pubescent, some what prickly; stem smooth; stipular pricliles straight ; leaflets elliptical-lanceolate, simply serrate, smooth when mature; flowers mostly by pairs.

HAB. Woods and copses. June-July. Shrub $3 f t$. high; fl. large red.

2. R. nitida: fruit subglnbose; calyx, peduncles, an $\mathbf{t}^{3}$ branches hispid; petioles somewhat hairy, un. armed; leaflets (7) oblong-lanceolate, on both sides smooth and shining.

HAB. Pennsylvania. +.

3. R. lucida : fruit depressed-globose, and with the peduncles somewhat hispid; petioles smonth, a little prickly; stem smooth; stipular prickles straight. leaflets ovate-lanceolate, rather obtuse, conrsely serrate, smooth and shining; flower's generally by pairs; segments of the calyx entire.

HAB. Mountain bogs. July. Shrub 3 ft. high; $f$ large, red.

4. R. gemella: fruit depressed-glohose, and with the peduncles glabrous ; flowers mostly by pairs, leaf. lets oblong, acute, opaque; petioles and under sur. face of the veins pubescent ; stipular prickles uncinate, by pairs.

HAB. Dry hills. June. A lore shrub; fl. large, reat

5. R. carolina: fruit globose, and, with the peduncles somewhat hispid ; petioles hairy, somewhat prickly: stem smooth; prickles uncinate; leaflets $(5-i$; oblong-lanceolate, acute, sharply serrate, glaucons beneath ; flowers in corymbs.

HAB. Swamps and wet thickets. June-July. Shrub 3-8 ft. high; prickles strung; corymnbs 57.fl. ; pet. obovate, large.

15. Rubiginosa : fruit ovate; peduncles and petioles slandular-hispid; stem smooth; branches anf 
prickles recurved; leaflets oblong, opaque, pubez cont above, ferruginous and glandular beneath.

HAB. Herlges, \&c. Shrub slend., climb.; leafl.51\%, swect-scent.; $A$. pale red.

328. RUBUS. Bramble. Rosacece.

* Frutescent.

1. R. Idcuis: leaves pinnate ; leaflets $3-5$, rhombicovate, acuminate, white and very downy beneath; petioles canaliculate; stem nearly erect, prickly. $H A B$. Borders of lakes.

2. R. villosus: pubesceni, hispid and prickly; stem angular ; leaflets in fives, digitate, elliptical, acuminate, scrrate, pubescent on both sides ; calyx shortacuminate; racemes loose, naked; pedicels solitary. HAB. Fields and hedges. June. Shrub 4-6 fto high; $f$. wh.; fr. large, blk.

3. frondosus: pubescence simple; racemes leafy, few-flowered; the upper flowers opening first; petals orhicular-ovate, approximate.

HAB. Road-sides. Fl. larger.

3. R. strigosus : unarmed; strongly hispid; leaflets in threes, or pinnate in fives, oval, obtuse at the base, marked with lines, and whitish-downy beneath ; the terminal one often subcordate; peduncles and calys bispicl.

HAB. Rocky hills. May. Shrub upright; raceme ferv-fl.; fr. red, agreeable.

1. R. euncifolius: branches, petioles, and peduncles pubescent; stem erect; slightly angular; leaflets ternate, cuneate-obovate, entire at the base, subplicate, tomentose beneath ; racemes loose; pedicels solitary, 1 -flowered.

HAB. Sandy fields and woods. June. Shrul $\approx f t$. high; fr. black, ovate, agreeable.

i. R. canadensis: smoothish; leares digitate, in tives and threes; leaflets phombic-larceolate, naked on both sides, acutely serrate; stem unarmed, with lanceolate bracts; pedicels elongated, 1-3-flow. ered; calyx 5-7-cleft.

HAB. Sandy woods and swamps. June. Stem sar. 
ment., procumb. or reclin.; leafl. thin; pet. short; fr. dark red.

6. R. occidentalis: branches and petioles glaucous and aculeate ; leaves ternate; leaflets ovate-acuminate, somewhat lobed, coarsely serrate, hoary tomentose beneath; petioles terete; racemes terminal. HAB. Rocky plices. May-Aug. Stems long, slenel.; racein. long; fr. biack, round.

7. R. hispidus: sarmentose-procumbent; stems, petioles and peduncles very hispid with rigid bristles; leaves ternate; leaflets incisely serrate, naked,; the intermediate one pedicellate.

HAB. Pennsylvania. June.

3. R. trivialis: sarmentose-procumbent; petioles ant peduncles aculeate-hispid, with the prickles recurved; stipules subulate; leaves ternate and quinate oval, acute, unequally serrate; pedicels solitary, elongated.

HAB. Stony fields. May-June. Stems long; leav. subsemperv.; fr. large, black.

9. R. odoratus: unarmed, erect, viscidly pubescent; leaves simple, acute, $3-5$-lobed ; corymbs terminal, divaricate; calyx appendiculate; petals nearly round.

HAB. Rocky places. June. Shrub 3-4 ft. high.; fl. large, purp.; fr. yellow, large.

* Somerehat herbaceous.

10. R. saxutilis $\beta_{\text {. canadensis : herbaceous, pubescent ; }}$ stems creeping; leaves ternate; leaflets rhombic, acute, incisely dentate, naked; the terminal one petiolate; flowers somewhat in threes; pedicels elongated.

HAB. Mountains. Fruit small, black.

11. R. obovalis: stem becoming a little woody, hispia with stiff hairs; leaves ternate ; leaflets roundobovate, serrate, naked; stipules setaceous; ra. cemes subcorymbed, few-flowered; bracts ovate; pedicels elongated.

HAB. Mountain swanps. May-July. Fr. with few acini, black, szerect.

12. R. Chumemorus: herbaceous, stnall; stem unarm 
ed, 1-flowered, erect; leaves simple, subren:form, with rounded lobes, petals oblong.

HAB. High mountains. $F l$. wh.; fro. yellow, or amber-col.

\section{DALIBAPDA: Rosacece.}

A. D. repens: villous; stolons creeping; leaves simple, cordate, crenate; peduncles 1-flowered.

HAB. Woods and mountains. June. 4. Scape filif.; $凡$. wh. ; pet. ovate.

2. D. fragarioides: leaves ternate; leallets ovate, incisely serrate, cuneate at the base; peduncles many-flowered.

HAB. Woods. May. Petiol. 3-4 in. long.; $f$ small, yellow.

330. DRYAS. Mountain avens. Rosacea

D. integrifolia: leaves elliptical-ovate, entire. HAB. High mountains. 4. Suffrut.; cespit.: sempvir.; $f$. zah., solit.

331. GEUM. Arens. Rosacece.

1. G. strictum: hairy; leaves all interruptedly pinnate; the terminal leaflet larger; leaflets ovate, dentate; stipules incised; calyx with 5 alternate segments, linear and short; flowers crect; petals roundish, a little longer than the segments; awns naled, uncinate.

HAB. Swamps. Aug. 24. Stem simp., 2 ft. high: fl. large, yellow, clust.

2. G. agrimonoides: very hairy; leaves all pinnate; leaflets nearly equal, unequaily and incisely dentate; stipules ovate, nearly entire; flowers erect; segments of the calyx subequal; petals oval, as long as the calyx.

IHAB. Rocky banks. \#. Fl. wh. Cfr. PotenTILLA confertiflora.

3. G. virginianum : pubescent ; radical and lower cauline leaves ternate; the upper lanceolate; stipules. ovate, nearly entire; flowers erect; petals shorter than the calyx; awns uncinate, naked, hairy, and twisted at the summit. 
HAB. Woods and thickets. June-July. 4. Stcm 2 ft. high; fl. pedunc., yell.-reh.

1. G. aibum: pubescent; radical leaves pinnate; stem leaves ternate, the upper one simple, 3-cleft; lower stipules incised; flowers erect; petals as long as the calyx ; awns uncinate, naked, hairy at the summit.

HAB. Banks of rivers, \&c. June-July. Stem $2 f t$. high; fl. wh., on short ped.

5. G. rivale: pubescent; stem simple; radical leaves interruptedly pinnate; cauline ones 3-cleft ; flowers nodding; petals as long is the calyx; awns plumose, nearly naked at the summit, minutely uncinate.

HAB. Bogs. May-June. Stem $1 \frac{1}{2} f t$. high; term. leafl. large; $f$ l. purp.

G. Peckii : somewhat hairy; stem few-flowered; radical leaves reniform; incisely toothed, and somewhat lobed; petioles elongated, with minute lenflets; petals roundish, longer than the calyx.

HAB. High mountains. July-Aug. 4. A span: high; fl. middle sized, yellow.

332. POTENTILLA. Rosacer.

* Leaves ternate.

3. P. tridentata: assurgent ; stipules subulate ; leaves ternate, oblong cuneiform, 3-toothed at the summit, smoothish above, pubescent beneath ; corymb loose, ferw-flowered; petals oblong-obovate, longet than the calyx.

HAB. MIountiins. June-July. Cespit, 3-6 in. high; lcav. coriac.; fl. weh.

2. P. hirsuta: stem erect, simple; leaves ternate; leaflets roundish, hairy, unequally dentate-serrate; teeth obtuse; petils shorter than the calyx.

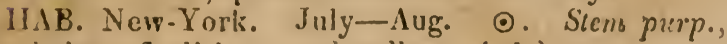
hairy; fl. dichot.; pale yellow; (woh.)

3. P. norvegica: stem erect, dichotomous above; leaves ternate, petiolate; leaflets oblong, acutely serrate, with spreading hairs; pedicels axillary; netals obovate, shorter than the calyx. 


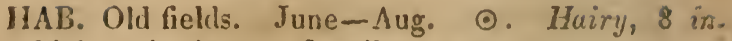
high ; stip. large; $f$. yellow.

\section{** Leaves digitare.}

A. P’. concudensis: whole plant silky villows; stem procumbent and ascending, somewhat branched; leaves quinate-obovate, incisely dentate; peduncles solitury, elongated; segments of the calyx linear-lanceolate; petals orbicular, nearly as long as the calyx.

1MAB. Fields and woods. April-May. 4. Stem 2-10 in. high; $f$. yellow ; recept. hairy.

6. P. simplex : stem sarmentose ; simple; leaves quinate; leaflets oblong-ovitl, coarsely serrate, hairy heneath; stipules incised; preduncles axillary, soli tary, elongated; petals roundish obcordate, a little longer than the calyx.

IIAB. Fields and woods. May-Aug. 4. Hairy; ped. $1 . f ; f$. yellore.

C. P. argentea: stem ascending; leaves quinate; leaflets cuneiform, incised, revolute on the margin; white and tomentose beneath; petals retuse, a little longer than the calyx.

HAB. Rocks and fields. June-Sept. \& Ofters sprcading; leav. gr. above; fl. yellow.

\section{*** Leaves pinnate.}

17. P. fruticost: stem fruticose; leares pinnate; leat lets oblong-lanceolate, very entire; petals longes than the calyx.

HAB. Bog meadows. Iune. A shrub, I ft. high: much branch.; $f$. yellow.

8. P. Anserina: stem creeping; leaves interruptedly pinnate; leaflets numerous, incisely and very acutely serrate, silky ; pedicels axillary, solitary, as long as the leaves; stipules many-cleft.

HAB. Wet meadows. June. 4. Stems long; leaft. 7-10 pairs, wh. beneath; $A$. yelloze.

@. P. supina: stem decumbent, dichotomous; leaves pinate; lenflets oblong, incisely serrate; pedun. cles axillary, solitary, 1-flowered.

AAB. Overflowed banks. June-Aug. ๑. $F l_{\text {: }}$ sinall, yellow. 
10. P. palustris: root creeping; stem ascending; leaves pinnate; leaflets lanceolate, acutely serrate; petals lanceolate, acuminate, much shorter than the calyx.

HAB. Swamps. June. 4. Stem $18 \mathrm{in.} \mathrm{high.}$ pubes.; leafl. 5-7; fl. large, dark purp.

11. P. pennsylvanica: erect, very soft, and somewhat whitish villous; leaves pinnate; leaflets oblong. obtuse, subpinnatifid, woolly; panicle straight, many-flowered; seginents of the calyx semioval. HAB. Pennsylvania, \&c. July-Aug. 4. Sterns num., 18 in. high; lcav. intcrrupt., pin.

12. P. confertiflora: viscous and very hairy; stem erect, nearly cimple ; leaves somewhat interruptedly pinnate; leaflets roundish-ovate, doubly and inciscly dentate, oblique; stipules ovate, acute, entire; flowers clusiered, subsessile ; lenflets of the calyx unequal, oblong, acute; petals obovate, longer than the calyx.

IIAB. Rocky hills. June. 4. Stem brownishpubes., 2 ft. ligh ; $f$. yell.-reh.

333. FRAGARIA. Strawberry. Rosaceo.

F. virginiana : leaflets broad-oval, smoothish above: the lateral oacs distinctly petiolate; hairs of the petiole sprearling; of the peduncles appressed; fructiferous; calyx spreading.

IIAB. Fields aud woods. May-June. If. Stem sarment.; $\Re_{\text {. }}$ wh. ; fr. red.

334. CAI,YCAN'THUS. Carolina Allspice. Rosacea:

C. levigatus: segments of the calyx linceolate leaves oblong or oval, gradually acuminate, somewhat rugose, smooth and green on both sides, branches straight, erect.

HAR. Mountains. Shrub 4-6 ft. high ; leav. nplo". f. large, purp., odor.

\section{POLYANDRIA.}

\section{MONOGYNIA.}

335. TILIA. Cal, inferior, 5-parted. deciduous. Pet. 
5. Cap. globose, 5-celled, 5-valved, opening at the base ; (by abortion 1-celled, 1-2-seeded.) 336. HELIANTHEIUM. Cal. 5-leaved; exterior leaflets smaller. Pet. 5. Caps. superior, 1celled, 3-valved; valves septiferous in the middle. Seeds angular.

337. HUDSONIA. Cal. tubular, 5-parted, unequal. Pet. 5. Stam. 15-30. Caps. 1-celled, 3-valved, 1-3-seeded.

338. PORTULACCA. Cal. inferior, bifid. Pet. 5. Caps. 1-celled, opening circularly. Recept. unconnected, 5-lobed.

339. TAlinUm. Cal.2-5-leaved, inferior. Pet. 5. Caps. 1-celled, 3-valved, many-seeded.- Recept. globose. Seed's axillate.

340. CHELIDONiUn. Cal. 2-leaved, deciduous. Pet. 4. Stig. small, sessile, bifid. Cajs. elongated, silique-form, 2-valved, 1-celled, linear. Seeds numerous, crested.

341. MIECONOPSIS. Cal. 2-leaved, deciduous. Pet. 4. Style distinct; stig. 4-6-rayed. Caps. oblong, mostly echinate, 1-celled, 4-6-valved: recept. slender.

342. ARGEMONE. Cal. 3-leaved, deciduous. Pet. 4-6. Stig. sessile, capitate, 4-7-rayed. Caps. ovate, angular, 1-celled, opening at the summit by valves; recep. linear. Seeds spherical, scrobicular.

343. SANGUinaria. Cal. 2-leaved, deciduous. 'Pet. about 8. Stig. didymous. Caps. oblong, 2valved, 1 -celled, acute at each end; valves deciduous; recept. 2 , persistent.

34. PODOPHYlluM. Cal. 3-leaved. Pet. abont 9. Stig. large, crenate, sessile. Caps? 1-celled, many-seeded, at length berry-like; recept. unilateral, large and pulpy.

345. ACT ÆA. Cal. 4-leaved, deciduons. Pet. 4, often wanting. Stig. sessile, capitate. Berry superior, 1-celled, many-seedet. Seeds hèmispherical.

346. SARRACENIA. Cal. double, persistent ; exterior smaller, 3-leaved; interior 5-leaved. Pet. 5 , deciduous. Stig. very large, persistent, pel- 
tate, covering the stamens. Caps. 5-celled, 5valved, many-seeded.

347. NUPHAR. Cal. 5-6-leaved. Pet. numerous: minute, inserted with the stamens upon the receptacle. Stig. disk-form, radiate. Pericarp berry-like, many-celled, many-seeded.

340. NYMPHÆA. Cal. 4-5-leaved. Pet.numerous, inserted upon the germens beneath the stamens. Stig. disk-form, radiate. Pericarp berry-like, many-celled, many-seeded.

\section{DI-PENTAGYNIA.}

349. DELPHINiUM. Cal. petaloid, deciduous, irre: gular.

350. ACONITUM. Cal. petaloid, irregular.

351. AQUILEGIA. Cal. 5-leaved, petaloid, deciduous. Petals 5, terminating below in a spurred nectary. Caps. 5, erect, acuminate with the styles. many-seeded.

352. CiMICIFUGA. Cal. 4-5-leaved. Pet. 4-8; (sometimes wanting.) Caps. 4-5, oblong, many-seeded.

353. ASCYRUM, Cul. 4-leaved; the 2 interior leaflets larger and cordate. Pet. 4. Stum. subpolyadelphous. Siyles 1-3. Caps. 1-celler.

354. HYPERICUM. Cal. 5-parterl; segments equal. Pet. 5. Stum. polyariclphous. Styles $1-5$. Caps. membranaceous, $1-5$-celled.

\section{POLYGYNAL.}

355. MAGNOLIA. Cal.3-leaved. Pet.6-9. Capsules 2-valved, 1-seeded, imbricated in a cone. Seedls herried, pendulous on a very long funiculus.

356. LiRiodendion. Cal. 3-leaved. Pet.6. Samarce sublanceolate, 1-2-secled, imbricated in a cone.

357. ASIIIINA. Cal. 3-parterl. Pet. 6, spreading, ovate-oblong; the interior smaller. Anth. subsessile. Berries several, ovate, sessile, manyserded.

353. CLFMATIS. Invol. 0, or calyciform. Cal.4-8- 
leaved, coloured. Pet. 0 , or very short. Seeds with a long, mostly plumose cauda.

359. ANEMONE. Invol. 3-leaved, distant from the flower ; leaflets divided. Cal. petaloid, 5-15leaved. Pet. o. Seeds numerous.

36u. THALICTRUM. Invol. 0. Cal. 4-5-leaved, petaloid. Pet. 0. Seeds without awns.

361. HYDRAS'IIS. Cal. 3-leaved, petaloid; leaflets ovate. Pet. 0. Berry composed of many 1 seeded grains.

362. CALTHA. Cal. coloured, 5-leaved; leaflets orbicular, petaloid. Caps. 5-10, compressed, spreading, 1-celled, many-seeded.

363. COPTIS. Cal. 5-6-leaved, coloured and petaloid, deciduous. Pet. small, cucullate. Capsules $5-8$, stipulate, diverging, ovate-oblong, 4-6seeded, rostrate.

364. TROLLIUS. Cal. 5-15-leaved, petaloid, deciduous. Pet. 5-20, small, tubular at the base. Cripsules sessile, subcylindrical, many-seeded.

B65. HEPATICA. Invol. 3-leaved, near the flower, resembling a calyx ; leaflets entire. Cal. 6-9leaved, petaloid, disposed in several series. Seeds without awns.

366. RANUNCULUS. Cal. 5-leaved. Pet. 5, with a nectariferous pore at the base, on the inside. Capsules numerous, ovate, shortly mucronate, 1-seeded, not opening.

36\%. BRASENIA. Cal. 3-leaved. Pet. 3-4. Pericarp oblong, 2-seeded. Seeds pendulous.

368. NELUMRIUM. Cal. petaloid, 4-6-leaved. Pet. numerous. Pericarps numerous, deeply imznersed in the upper surface of a turbinate receptcicle; (torus.) Seed large, round, solitary.

\section{POLYANIRIA.}

\section{POLYGYNIA.}

335. TILIA. Lime, or Linden. Tiliacece.

1. T. glabra: leaves round-cordate, abruptly acumibate, acutely serrate, subcoriaceous, smooth; pe. 
tals truncate at the apex, crenate ; style as long as the petals, equal ; fruit ovate, subcordate.

HAB. Woods. June. A large tree; $f$. cymose; yell.-wh., ped. winged.

2. T. pubescens: leaves truncate at the base, oblique, denticulate-serrate, pubescent beneath; petals emarginate; styles longer than the petals; fruit globose, smooth.

HAB. Banks of rivers. June. A large tree.

3. T. laxiflora: leaves cordate, gradually acuminate, sparingly toothed, membranaceous, smooth ; panicle loose; petals emarginate; styles longer than the petals ; fruit globose.

HAB. Near the sea-coast. June.

\section{HELIANTHEMUM. Cistinece.}

1. H. canadense: without stipules; erect, hairy; leaves erect, linear-lanceolate, flat, paler beneath ; raceme terminal, few-flowered; segments of the calyx broad-ovate; capsule shorter than the calyx. HAB. Dry fields and woods. June. 24. A foot high; fl. yellow; cal. hairy.

2. H. ramulifiorum: without stipules, erect, pulverulent-tomentose; leaves oblong, aciste; margin a little revolute, whitish beneath; racemes very short; segments of the calyx roundish, pulverulent ; capsule globose, as long as the calyx.

LAB. Sandy fields. 4. July. Fl. yellow; style very shorl; cal. hairy.

3. I1. corymbosum: suffrutescent, without stipules; erect, branched, pubescent; leaves alternate, lanceolate, hoary-tomentose bencath; flowers crowded, in fistigiate corymbs; segments of the cilly $x$ ovate, acute; capsule scarcely as long as the calyx.

IIAB. Sandy fields. June-Aıg. Fl. very small. often apet.

337. HUDSONIA. Cistinece.

1. H. ericoides : pubescent; suberect; branches elongated, leaves filiform, subulate, somewhat imbricate; peduncles numerons, elonguted; calyx cy- 
lindrical, obtuse; capsule pubescent, 1-seeded : valves oblong.

HAB. Pine barrens. May-June. 万. Stem 46 in. high ; leav. persist. ; fl. sm. yellow.

.H. tomentosn: cespitose, hoary-pubescent ; leaves minute, closely imbricate, ovate, acute ; flowers aggregated, subsessile; calyx subcylindric; segments obtuse ; capsule smooth.

HAR. Sea-shore. June. 万. Stem 6 in. high, intricate; $f$. num., yellow.

338. PORTUlaCCA. Purslane. Portulacea.

P. oleracea: leaves cuneiform, smooth; flowers sessile.

HAB. Cultivated grounds, \&c. May-Aug. (). Spreading, succul.; fl. yellow. §.

\section{TALinUm. Portulacece.}

T. teretifolium: leaves terete, subulate, fleshy: scape cymose ; flowers pedunculate, polyandrous : calyx 2-leaved.

HAB. Rocks. July. 3. Subcespit.; leav. crowed. ; rad.; fl. purp.

340. Chiflidonium. Celandine. Papaveracea.

C. ancjus: leaves pinnate, lobed; segments rounded ; umbels axillary, pedunculate ; petals elliptical, entire.

HAB. Along fences, \&c. May-Oct. 4. Plant yielding an orange juice, branch.; leav. glauc.; ff yellow. \$.

311. MECONOPSIS. Papaveracere.

11. diphylla: leares 2, sessile, hairy ; lobes rouncled and obtuse, subundulate : capsule 4-valved, echinate.

HAB. Shady woods, \&c. May. 4. A foot high: glauc. and subpilose; flo yellow.

342. ARGEJONE. Prickly poppy. Papaveracece.

A. mexicana. 
IIAB. Banks of rivers. July. ๑. Leav. pinnatif., spiny; $f$. axill. and term., large, yellow or white.

343. SANGUINARIA. Blood-root. Papaveracece.

S. canadensis,

HAB. Fertile wonds. April-May. 4. Root tab. with a bitter juice; leav. rad., renif.; fl. large, zh.h., solit.

344. PODOPHYLLUM. May-apple. Podophyllaces.

P. peltatum : stem erect, 2-leaved; leaves peltate. HAB. Woods. May. ४. Stem 1' ft. high; lear. lobed; fl. sol. weh.; fr. ovate.

345. ACT ÆA. Bane-berry. Ranunculacea.

A. americana: leaves twice and thrice ternate; raceme ovate; petals shorter than the stamens; berries ovate-oblong.

a. alba: petals truncate; pedicels of the fruit thicker than the peduncle; berries white.

ß. rubra: petals acute; pedicels of the fruit slender ; berries red.

HAB. Rocky woods. May. 4. Stcm $2 f t$. high : leafl. ovate-lan., incis.; fl. wh.

\section{SARRACENIA. Side-saddle flower.}

S. purpurea: leaves much shorter than the scape. inflated; ventral wing arched; appendix erect. broad-cordate, undulate, not mucronate.

HAB. Sphagnous swamps. June-July. Leav. all rad., large and tubular, open at the top; scape $1 \mathrm{ft}$. high; fl. large, sol., purp.

ß. - : flowers yellow.

347. NUPHAR. Yellow Pond-lily. Nymphacacec.

1. N. adzena: calyx 6-leaved; petals numerous ; fruit sulcate; leaves cordate, with divaricate lobes: petioles semicylindrical. 
HAB. In water. June-July. 4. Leav. upright or floating; fl. large, depress, yellow.

2. N. Kalmiana: calyx 5-leaved; stigmas incised, 812-rayed ; leaves cordate, with approximate lobes ; petioles roundish.

$\mathrm{HAB}$. In water. July-Aug. 4. Leav, and $f$. small; cal. equal.

348. NYMPHÆA. Water-lily. Nymphocacece.

N. odorata: leaves orbicular-cordate, entire; nerves and veins prominent; calyx 4-leaved, equal to the petals; stigma 16-20-rayed; rays inflexed.

HAB. In water. June-July. 4. Leav. float.: fl. large, wh., odorous.

\section{DI-PENTAGYNIA.}

349. DELPHINIUM. Larkspur. Ranunculacex.

1. D. exaltalum: petioles not dilated at the hase; leaves flat, 3-cleft below the middle ; lobes cuneiform, 3-cleft at the apex, acuminate ; lateral ones often 2-lobed ; raceme straight ; spur longer than the calyx ; capsules 3.

HAB. Rncky woor's. July 4. Stem 2-4 ft. high; fl. öluse; spur straight.

2. D. azureum: petioles slightly dilated at the base; leaves 3-5-parted, many-cleft; lobes linear ; raceme straight; petals densely bearded; flowers on short pedicels.

HAB. Woods. May. 4. Stem \& ft. high; fl: large, light blue.

350. ACONITUM. Wolf's-bane. Ranuncularece.

A. uncinatum: panicle rather loose, with diverging branches ; galea conical ; spur inclined, somewhat spiral; styles 3-5; leares 3-loberl; lobes equal. HAB. Mountain swamps. Sept. 4. Fl. large, blue.

351. AQUILEGIA. Columbine. Ranunculacer.

A. canadensis: spurs straight; styles and stamens exserted; calyx rather acute, longer than the pe- 
tals ; division of the leaves 3-parted, rather obtuse, incisely toothed.

HAB. Rocks. April-May. 4. Fl. pend., scarl. ext., yell. int.; fr. erect.

\$52. CIMICIFUGA. Black Snake-root. Ranunculacee.

C. racemosa: leaves ternately decompound; leaflets ovate-oblong, incised and dentate; racemes paniculate, elongated; flowers with one style; capsule ovate.

HAB. Rocky woods. June-July. 4. Stem 48 ft. high; $\lambda_{\text {. wh. ; pet. } 0 .}$

353. ASCYRUM. St. Peter's-wort. Hypericinece.

1. A. Crux-Andrece: stems numerous, assurgent, suffruticose ; leaves lanceolate-oblong, obtuse; interior leaflets of the calyx suborbicular; pedicels bibracteate; flowers sessile, with 2 styles.

HAB. Pine barrens. July-Aug. Stems 6-s in. high fl. num., yellow ; pet. narrow.

2. A. stans : stem fruticose, winged, straight; leaves ovate-elliptical, obtuse, glancous; interior leaflets of the calyx cordate, orbicular; stamens united at the base, styles 2.

HAB. Pine barrens. Aug. Stem $1 \mathrm{ft}$. ligh; branch. straight; fl. yellow.

354. HYPERICUM. St. John's-wort. IIypericinece.

* Styles 5; Stam. numerous, indefinite; flowers yellow.

1. H. ascyroides: smooth; stem quadrangular, winged at the base; leaves sessile, oblong-lanceolate, acute; calyx ovate-lanceolate; styles as long as the stamens.

HAB. Low grounds. July. Siem 2 ft. high, simp. or branch.; fl. and leav. large.

a. II. Kalmianum: frutescent, much branched; branches qualrangular; leavez linear-lanceclate; flowers $3-7$, in a terminal corymb; calyx lanceolate, rather obtuse.

HAB. Wet rocks. August. Stem 2-4 ft. high; lcav. num., fascic., ofien revol. 
* Styles 3 ; stamens numerous, indefiniie; flowers yellow.

\section{$\dagger$ Frutesrent.}

3. H. prolificum: branches ancipital; leaves lanceolate-linear, rather obtuse, corymbs axillary and terminal, few-flowered; calyx lanceolate.

HAB. Pennsylvania. July.

1. H. galioides: branches quardrangular ; leaves linear, sessile, revolnte on the margin, punctate ; panicles terminal, dichotomous, and divaricate ; caly x linear ; styles often united.

HAB. Wet santly places. July-Sept. Stem $2 \mathrm{ft}$. high.

\section{††. Herbaceous.}

5. H. perforatum: stem ancipital; leaves obtuse, oblong, with pellucid punctures; flowers paniculate; calyx lanceolate; styles diverging.

HAB. Fields. June-Aug. 4. A foot high; branch.; anth. black-punctate. $\S$.

6. H. punctatum: stem terete, black-punctate; leaves oblong-oval. obtuse, amplexicaul ; flowers corymbed, punctate ; calyx lanceolate.

HAB. Meadows and woouls. June-Aug. 4. Stern 2 ft. high; branch.; fl. small.

7. H. quinquenervium : erect, much branched, smooth; stem quadrangular ; leaves ovite, subcordate, obtuse, sessile, about 5-nerved; corymb dichotomous; calyx linear-lanceolate, longer than the petals.

HAB. Wet places. June-Sept. ๑. Stem 6-10 in. ligh; fl. very small; yelloz.

3. H. canadense : stem erect and straight, 4-winged; leaves linear, attenuate at the base, rather obtuse ; panicle elongated, dichotomous ; calyx lanceolate; styles very short; capsule conical.

HAB. Sandy places. June-July. ○ . Stem 610 in. high; $f$. minute; caps. red.

9. H. angulosum : erect ; stem quulrangular; leaves distant, oblong-lanceolate, subamplexicaul, acute ; panicle dichotomous, with the flowers alternate: calyx lanceolate, acute, subcarinate. 
HAB. Cedar swamps. Aug. 4. Stem 1-1 $\frac{1}{2} f t$. high; fl. copper-colour.

10. H. Sarothra: erect, much branched above; branches setaceous; leaves minute, subulate, appressed; flowers terminal, subsolitary; stamens few; capsule 1-celled.

IIAB. Sandy fields. June-Aug. $\odot$. Stem $3-6$ in. high; leav. inconspic.; $f$ minute.

*** Caly: of 5 equal leares; styles 3 ; stamens numerous, somezohat definite; $(9-15-18$.$) distinctly$ polyadelphous.

11. II. virginicün: stem terete, leaves oblong, amplexicaul, punctate, very obtuse, flowers pedunculate, in terminal and axillary peduncles; calyx lanceolate; stamens mostly 9.

HAB. Swamps. July-Sept. 4. Stem $1 \mathrm{ft}$. high; $f$. middle-sized, reddish.

\section{POLYGYNIA.}

\section{MAGNOLIA. Magnoliacece.}

1. M. acuininata: leaves deciduous, oval, acuminate, pubescent beneath ; flowers 6-9-petalled; petals obovate, rather obtuse.

IIAB. Mountain valleys. June-July. Tree middle-sized; $f l$ yell.-gr.

2. M. tripetala: leaves deciduous, oblong-lanceolate, acute, spreading; pubescent when young, smooth when mature; petals $9-12$, rather acute, the 3 exterior ones reflexed.

HAB. Mlountain woods. June. A small tree; leav. and fl. large.

3. M. glauca: leaves perennial, elliptical, obtuse ; glaucous beneath; flowers $9-12$-petalled; petuls obovate, concave.

HAB. Swamps. Niay-June. A small tree; leav. $2-3$ in. long ; $f$. odiorous.

356. Lifiodindion. Tulip.tree. Magnoliacer.

I. Tu! ipifora. 
HAB. Woods. July. A large tree; leaz. 4-lobed, truncate; $f$ l. large, sol., yell.-gr.

\section{ASIMINA. Anonacere.}

A. triloba: leaves oblong-cuneate, acuminate, smoothish; flowers on short peduncles; exterior petals suborbicular, four times as long as the petals.

IIAB. Banks of rivers. May. A large shrub; $f$. dark br.; fr.fleshy, large, edible.

358. CLEMATIS. Virgin's-bower. Ranunculacece.

1. C. virginiana: climbing; leaves ternite; leaflets ovate, subcordate, incisely toothed and lobed; acute; flowers paniculate, dicecious.

HAB. Shady thickets. July-Aug. h. Stem long; fl. white, in large dichot. pan.

2. C. Viorna: climbing; leaves pinnately compound; segments ovate, entire, or 3-lobed, acute ; flower solitary, campanulate; leaflets of the calyx thick, acuminate, reflexed at the apex.

11 AB. In copses, \&c. June. Ћ. Fl. large, violet, nodding; lear. smooth.

3. C. ochroleuca: herbaceous, erect, simple, pubescent; leaves simple, ovate, very entire; the younger ones with the calyx silky; flower terminal, pedunculate, solitary, nodding.

IIAB. Dry copses. June. 4. A foot high; $f$. yeil.-※.h.; fr. erect.

$†$ Calyx 4-leaved; petals numerous, minute. Atragene.

1. C. verticilluris: leaves verticillate in fours, ternate; leaflets cordate, nearly entire; peduncles 1 -flowcred; petals acute.

HAB. Mountains. MIay-June. $\boldsymbol{~}$. Climbing; $f$. rery large, purp.

359. ANEAIONE. Wind-flower. Ranunculaced.

1. A. nemorosa: $\beta$. quinquefolia: leaves ternate; segmenis 5-parted, incisely dentate, lanccolate, acute ; involucrum similar, petiolate; stem 1.flowered; calyx 6-leaved; capsules awnless. 
HAB. Woods. April-May. u. Root black, tub.; slem 6 in. high; $f$ l. wh. or purplish.

2. A. lancifolia: leaves ternate; leaflets lanceolate, crenate-dentate; calyx 5-leaved, leaflets ovate, acute; stem 1 -flowered.

HAB. High mountains. May-July. 4. Fl. wh.; $f r$. ovate, with a short uncinate style.

3. A. pennsylvanica: leaves 3-parted; lobes oblong, incisely toothed at the apex ; involucrum similar, sessile, bearing several pedicels, one of which is naked and 1-flowered, the others involucellate; fruit pubescent.

HAB. Meadors:- June-July. 4. A foot high ; pedunc. elong.; $f$. yell.-zoh., large.

4. A. virginiana: leaves ternate; segments 3-cleft, acuminate, incisely toothed; involucrum similar. petiolate; leatlets of the calyx acuminate; fruit oblong.

HAB. Woods. July. 4. Sten 2 ft. high; fl. gr.yell.; cal. hairy; caps. woolly.

360. Thalictrum. Meadow-rue. Ranunculacece.

* Stamens longer than the calyx.

1. $T$. dioicum: very smooth; leaves decompound; leaflets roundish, with obtuse lobes, glaucous beneath ; filaments filiform; flowers dioecious.

HAB. Rocky woods. April. 4. Stem $2 \mathrm{ft}$. high; fl. panic., rose-col.; fr. oval, striate.

2. T. pubescens: leaves decompound; leaflets ovate, 3-lobed, minutely and densely pubescent beneath ; margin revolute; filaments filiform ; flowers polygamous.

HAB. Wet meadows. June-July. 4. Stem 4$5 \mathrm{ft}$. high; $\mathrm{fl}$. in large pan. ; cal. decid.

3. T. Cornuti: leaves decompound; leaflets ovate, ol)tusely 3-lobed, glaucous and a little pubescent on the veins beneath; flowers diocious; filaments clavate; fruit sessile, striate.

IIAB. Wet meadows. June-July. Stem $2-5 f l$. high; $\lambda$. gr.-yell. +.

4. 'T. rugosum: leaves decompound; leaflets ovatelanceolate, rugose, reined, obtusely lobed; flowers dio:cious, lilaments filiform. 
HAB. Swamps. June-Aug. Stem 3-5 ft. high; fl. in large pan., wh.

5. T. purpurascens: leares compound, shorter than the stem; leaflets roundish, 3-cleft and incised; panicles nearly leafless; flowers cernuous; stamens coloured.

HAB. Dry hills. May-June. 4. Plant small; stem and filam. purp. +.

** Stumens shorter than the petaloid calyx.

6. T. anemonoides: root tuberous; flowers umbelled; floral leaves petiolate, resembling an involucrum; flowers perfect ; calyx 8-10-leaved.

HAB. Woods. April-May. 4. Stem 6-8 in. high; leafi. smooth; fl. large, wh.

361. HYDRASTIS. Yellow-root. Ranunculacece.

H. canadersis.

HAB. Rocky woods. May. 4. Stem $6 \mathrm{in}$. high, 1-2-leav.; leav. incis., pubes.; fl. solit., red-roh., fr. fleshy, red.

362. CALTHA. Marsh-marigold. Ranunculacea.

1. C. palustris : stem erect; leaves cordate, suborbicular, obtusely crenate.

HAB. Swamps. April-June. 4. A foot high, dichot.; leav. petiol.; fl. large, yellore.

2. C. integerrima : stem erect, corymbed; leaves orbicular-cordate, very entire, with the sinus closed; floral ones sessile, reniform, obscurely crenate at the base; leallets of the calyx obovate.

IIAB. Wet meadows, \&c. May-July. 4. F\%. corymb., smaller than in No. 1.

3. C. parnassifolia: stem erect, 1-flowered, 1-leaved; radical leares, cordate-ovate, very obtuse; manynerved; leallets of the calyx elliptical.

HAB. Cedar swamps. June-July. 24. Fl. middle-sized, deep yellow.

1. C. Alabellifolia: stem procumbent, many-flowered; leares dilated-reniform; lobes widely spreading, acutely dentate; leaflets of the calyx obovate; capsules uncinate. 
HAB. Mountain springs. July-Aug. 2f. A foot high ; fl. middle-sized, yellow.

\section{COPTIS. Ranunculacec.}

C. trifolia: leaves ternate; leaflets obovate, obtuse, dentate, obscurely 3-lobed; scape 1-flowered.

HAB. Swamps. May-June. 24. Leav. on long pet.; scape 6 in. high; $f l$. wh.

\section{TROLLIUS. Rununculacee.}

T. laxus : calyx spreading; leaflets $5-10$, oblong; petals (nectaries) shorter than the stamens.

HAB. Wonds. June. 24. Stem 1 foot high; leaves palm.; fl. large, yellow.

365. HEPATICA. Liverwort. Ranunculacee.

H. triloba: leaves cordate, 3-lobed ; lobes entire ; petioles and scape hairy.

\%. obiusa: lobes of the leaves rounded, obtuse.

B. acuta: lobes of the leaves acute.

HAB. $\alpha$. In woods: $\beta$. On mountains. AprilMay. 24. Scape 1.fl.; fl. blue.

366. RaNUNCULUS. Crowfoot. Ranunculacece.

*. Pericarps transversely rugose-striate; petals white.

1. R. aquatilis : $\beta$. capillaceus : :tem filiform, floating; leaves all immersed, and filiformly dissected; petals obovite, longer than the calyx.

HAB. In brooks, \&c. July-Aug. 24. Stem long; leav. petiol.; fl. small; cal. pubes.

**. Pericarps smooth, short, ovate, collected into a rounclish head; fluwers yellow.

†. Leaves undivided.

9. R. I ingua : leaves lanceolate, subserrate, sessile, semiamplexicaul; stern erect, smooth. 
HAB. Borders of lakes, \&c. July. 4. Stem 2$3 \mathrm{ft}$. high, succul.; fl. large, yeliow.

3. R. Flammula : leaves smooth, linear-lanceolate, or subovate, nearly entire, the lower oues petiolate; stem more or less decumbent, rooting; peduncles opposite to the leaves.

HAB. Swamps. June-July. 24. Stem 12-18 in. high, succul.; fl. smaller than No. 2.

4. R. pusillus: leaves all on long petioles; the inferior ones ovate, subdentate; superior linear-lanceolate ; stems numerous, erect; petals mostly 3 , as long as the calyx.

HAB. Wet places. July. 24. A span high; rad. leav. cord.; fl. minute.

5. R. Cymbalaria: radical leares petiolate, smooth, somewhat fleshy, cordate-reniform, coarsely crenate; scapes naked, elongated, $1-4$-flowered; petals linear, as long as the calyx.

HAB. Salt marshes. -July-Aug. 2\%. Scapes 26 in. high, often stolonif.; fl. sm. ; fr. oblong.

๑. R. reptans: $\beta$. filiformis: leaves linear-subulate; stens filiform, creeping, geniculate; joints 1 . flowered.

HAB. River banks. July-Aug. 24. Stems 6$10 \mathrm{in}$. long; fl. small; fr. very smooth.

\section{††. Leaves divided.}

7. R. abortivus : leaves smooth; radical ones petiolate, cordate-orbicular, crenate; stem leaves ternate and 3-cleft, with linear segments ; calyx smooth, a little longer than the petals.

HAB. Rocky woods. April-June. 2f. A foot high; fl. small; fr. ovate, compress.

3. R. sceleratus: leaves smooth; radical ones petiolate, 3-parted; divisions 3-lobed, obtuse, subincised ; superior 3-parted, with oblong-linear entire lobes ; calyx pubescent ; fruit linear-oblong. HAB. Ditches, \&cc. May-Aug. 24. A foot high, much branch.; stem succul.; $f l$ s smull. 
3. R. multifidus: floating; leaves all cleft into numerous capillary segments, with axillary leallets; petals $5-8$, obovate, twice as long as the calyx ; nectary concare.

HAB. Stagnant waters. May-June. 4. Stem 3-4 ft. long; fl. large, shining.

10. R. acris: leaves mostly pubescent, 3-parted; lobes incisely toothed, acute ; upper ones linear; stem erect, many-flowered; peduncles not sulcate ; calyx spreading, subvillous.

HAB. Wet meadows. June. 4. Stem $2 f t$. high, pubes.; fl. large; cal. reflex. §.

11. R. hispiclus: erect, branched; stem and petioles with stiff spreading hairs; leaves ternate; leaflets acutely lobed; pubescence of the pedicels appressed; calyx appressed.

HAB. Wet places. May. 2f. Stem $1 \frac{1}{2} f t$. high, very hairy; fl. large.

12. $R$. recurvarus: erect; stem and petioles with spreading hairs ; leaves 3-parted, hairy ; segments broad-oval, subincised; the lateral ones 2-lobed; calyx reflexed; petals lanceolate; pericarps uncinate.

HAB. In woods. May-July. 4. A foot high; leav. subpentang.; fl. very small.

13. R. pennsylvanicus : erect; stem and petioles very hairy; the hairs rigid and spreading; leaves ternate, villous; segments subpetinlate, acutely 3-lobed, incisely serrate; calyx reflexed; pericarps with a straight style.

HAB. Wet mearlows. June-Aug. 4.? Stem 18 in. high; branch.; $A$ l. large; fr. in obl. heads.

14. R. fascicularis: erect, branched; leaves pubescent, ternate; the middle segment deeply 3 -cleft ; lateral ones remotely 3 -lobed; calyx spreading? villous, shorter than the petals.

HAB. Rocky woods. April-May. H. Rootfascic.; fl. large; nect. flat, cuneif.

15. R. bulbosus : hairy; radical leaves ternate; leaflets petiolate, 3-cleft, incisely dentate, stem erect, bulbous at the base; calyx reflexed; peduncles sulcate. 
HAB. Pastures, \&c. May-July. 4. Stem 1218 in. high; fl. large; plant acrid.

16. R. marilandicus: stem erect, somewhat branched, soft-hairy ; leaves smoothish, ternate ; leaflets 3 lobed ; lobes oblong, acute, incisely-dentate ; calyx smooth, spreading, shorter than the petals. HAB. Woods. May-July. 4. Pericarps compres., acum., with a straight style.

17. R. repens: leaves ternate; leaflets cuneate, 3 lobed, incisely-dentate; middle one petiolate; main stems prostrate; flowering ones erect; peduncles sulcate; calyx appressed.

HAB. Wet meadows. June-Sept. 4. Stem 1-2 ft. high; fl. middle-sized.

367. BRASENIA. Water-target. Podophyllacece.

B. Hydropeltis.

HAB. Ponds. July-Aug. if. Floating; leaves oval, centrally peltate, very entire; purp. and gelat. beneath; $f$. sol., purp., on long pedunc.

363. NELUMBIUM. Water-chinquepin. Nympheacece.

N. luteum : corolla many-petalled; anthers produced into a linear appendage at the extremity; leaves peltate, orbicular, very entire.

$\mathrm{HAB}$. In lakes. July. 4. Petioles and pedunc. scab.; fl. very large, yellow.

\section{DIDYNAMIA.}

\section{GYMNOSPERMIA.}

* Calyx mostiy 5-cleft, subregular.

369. TEUCRIUM. Upper lip of the Cor. wanting, but a fissure in its place, through which the stamens - are exserted.

370. MENTHA. Cor. subregular, 4-lobed; the broader segment emarginate. Stam. erect, distant.

371. ISANTHUS. Cal. subcampanulate. Cor. 5 parted; tube straight and narrow; segments of 
the border equal. Stam. subequal. Stig. linear, recurved.

372. HYSSOPUS. Lower lip of the Cor. 3-parted; middle segment crenate. Stam. straight, distant. 373. NEPFiTA. Cul. arid, striate. Tube of the Cor. rather long, intermediate segment of the lower lip crenate, margin of the orifice reflexed. Stam. approximate.

374. LAMIUM. Upper lip of the Cor. vaulted, entire; lower lip 2-lobed, toothed on each side.

375. GALEOPSIS. Upper lip of the Cor. vaulted, notched; lower lip 3-lobed, 2-toothed above.

376. STACHYS. Upper lip of the Cor. vaulted, lower lip 3-lobed; the lateral lobes reflexed. Stam. reflexed to the sides after flowering.

377. LEONURUS. Cal. 5-angled. Upper lip of the Cor. entire, flat, erect; lower lip 3-parted; middle segment eutire. Anth. sprinkled with shining dots.

378. GLECHOMA. Upper lip of the Cor bifid. Anth. approaching each other in pairs, and forming at cross.

379. MARRUBIUM. Cal. 10-ribbed. Upper lip of of the Cor. linear, straight, cleft.

379. PYCNANTHEMUM. Heads surrounded by an involucrum of many bracts. Cal. tubular, striate. Upper lip of the Cor. nearly entire; lower lip 3-cleft. Stain. nearly equal, distant; cells of the Anth. parallel.

$$
\text { * Calyx iilabiate. }
$$

381. CLINOPODIUM. Whorls surrounded by a setaceous involucrum.-Upper lip of the Cor. flat, emarginate.

382. ORIG INUM. Flowers collected into a dense, 4sided spike. Upper lip of the Cor. straight, flat, emarginate.

383. DRACOCEPHALUM. Orifice of the Cor. inflated; upper lip concave. Stam. unconnected. 384. PRUNELLA. Upper lip of the Cal. dilater. Filam. forked, one of the points antheriferous.

335. SCUTELLARIA. Upper lip of the C'al. corering the fruit like an operculun. 
386. TRICHOSTEMA. Cal. resupinate. Upper lip of the Cor. falcate. Stam. very long, and incurved.

\section{ANGIOSPERMIA.}

\section{*. Calyx 5-cleft.}

387. PHRYMA. Cal. cylindric; upper lip longer, 3cleft; lower lip bidentate. Upper lip of the Cor. emarginate; lower much larger. Seed so. litary.

388. VERBENA. Cal. with one of the teeth truncate. Cor. infundibuliform; limb 5-cleft, unequal. Stam. 2-4. Seeds 2-4, enclosed in a thin evanescent pericarp.

389. ZAPANIA. Flowers capitate. Cal. 5-toothed. Cor. 5-lobed. Stam. 4, fertile. Stig. peltately capitate, oblique. Seeds 2, at first enclosed in an evanescent pericarp.

390. HERPESTIS. Cal. unequal, bibracteate at the base. Cor. tubular, subbilabiate. Stam. included, all fertile. Caps. 2-valved, 2-celled; dissepiment parallel with the valves.

391. LIMOSELLA. Cal. 5-cleft. Cor. 4-5-lobed, equal. Stam. approximating by pairs. Caps.2-1 valved, partly 2-celled, many-seeded.

392. SCROPHULARIA. Cor. subglobose, resupinate, shortly bilabiate, with an internal intermediate scale. Caps. 2-celled.

393. BIGNONIA. Cal. 5-toothed, cyathiform, partly coriaceous. Cor. campanulate, 5 -lobed, ventricose on the under side. Silique 2-celled. Seeds membranaceously winged.

394. RUELLIA. Cal. often bibracteate. Cor. subcampanulate; border 5-lobed. Stam. approximating by pairs. Caps. attenuated at each extremity, bursting with elastic teeth. Seeds few. 395. BUCHNERA. Cal. 5-toothed. Tube of the Cor. slender; border 5-cleft, nearly equal; lobes cordate. Caps. 2-celled.

396. ANTIRRHINUM. Cal. 5-parted. Cor. personate or ringent, with a prominent or spurred 
nectary at the base. Caps. 2-celled, bursting at the summit, with reflected teeth.

397. COLLINSIA. Cal. 5-cleft. Cor. bilabiate, orifice closed; upper lip bifid; the lower trifid; intermediate segment carinately saccate, and closed over the declinate style and stamens. Caps. globose, partly 1-celled, and imperfectly 4-valver. Seeds 2-3, umbilicate.

398. GERARDIA. Cal. half 5-cleft, or 5-toothed. Cor. subcampanulate, unequally 5-lobed; segments mostly rounded. Caps. 2-celled, opening at the summit.

399. PEDICULARIS. Cal. ventricose, half 5-cleft. Upper lip of the Cor. arched, laterally compressed, emarginate. Caps. 2-celled, oblique, mucronate. Seeds angular.

400. MIMULUS. Cal. prismatic, 5-toothed. Cor. ringent; upper lip reflexed at the sides; palate of the lower lip prominent. Stig. thick, bifid. Caps. 2-celled, many-seeded. Seeds minute.

101. CHELONE. Cal. 5-parted, with 3 bracts. Cor. ringent, ventricose. Sterile filam. shorter than the rest; anthers woolly. Caps. 2-celled, 2 valved. Seed.s membranaceously margined.

402. PENTSTEMON. Cal. 5-leaved. Cor. bilabiate, ventricose. The fifth steril filament longer than the rest, and bearded on the upper side. Caps. ovate, 2-celled, 2-valved. Seeds numerous, angular.

403. MARTYNIA. Cal. 5-cleft. Cor. ringent. Caps. ligneous, corticate, 4-celled, 2-valved, each of the valves terminating in a long hooked beak.

$$
\text { ** Calyx 4-cleft. }
$$

404. SCHWALBEA. Cal. ventricose-tubular; upper segment shortest; the lower large and emarginate. Cor. ringent; upper lip entire, arched. Caps. ovate-oblong, 2-celled, 2-valved; dissepiinent produced by the inflected margin of the valves, and parallel with the longitudinal receptacle. Seeds numerous, imbricated, linear, vinged. 
105. EUCHROMA. Cal. ventricose, 2-4-cleft. Cor。 bilabiate; upper lip very long and linear, embracing the style and stamens. Anth. linear, with unequal lobes, all cohering in the form of an oblong disk. Caps. ovate, compressed, 2 celled. Seeds numerous, surrounded with a membranaceous inflated vesicle.

406. BARTSIA. Cal. 4-cleft. Cor. ringent; upper lip concave, entire. Anth. equally lohed, uncombined. Caps. 2-celled. Seeds angular.

40\%. MELAMPYRUM. Cal. 4-cleft. Upper lip of the Cor. compressed ; margin folded back ; lower lip groored, trifid, subequal. Caps. 2-celled, oblique, opening on one side; cells 2 -seeded. Seeds cartilaginous, cylindric-oblong, smooth.

408. OROBANCHE. Cal. 4-5-cleft; segments often unequal. Cor. ringent. Caps. ovate, acute, 1-celled. Seeds numerous. A gland beneath the base of the germen.

409. EPIPHEGUS. Polygamous.-Cal. abbreviated, 5-toothed. Cor. of the infertile flower ringent, compressed, 4-cleft; lower lip lat : corolla of the fertile flower minute, 4-toothed, deciduous. Caps. truncate, oblique, 1-celled, imperfectly ${ }_{2}$ valved, opening only on one side.

\section{DIDYNAMIA.}

\section{GYMNOSPERMIA.}

369. TEUCRIUM. Germander. Labiatce.

1. T. canadense: hoary-pubescent; leaves ovate-lanceolate, serrate, all petiolate; stem erect; spike verticillate, crowded, long.

HAB. Low grounds. July-Aug. 4. Siem 12$18 \mathrm{in.} \mathrm{high;} \mathrm{bracts} \mathrm{longer} \mathrm{than} \mathrm{the} \mathrm{cal.;} \mathrm{fl.} \mathrm{purp.}$

2. T. virginicum: pubescent; leaves ovate-oblong, serrate; the upper ones subsessile; stem erect; spikes verticillate, crowded; bracts longer than the calyx.

HAB. Low grounds. June-Aug. 4. Resem. bles the foregoing. + 


\section{MENTHA. Mint. Labiatce.}

1. M. viridis: spikes interrupted; leaves sessile, lanceolate, acute, naked; bracts setaceous, and with the teeth of the calyx somewhat hairy.

HAB. Low grounds. Aug. 4. A foot high; leav. smooth; spik. long; fl. purp. $\S$.

2. MI. canadensis: flowers verticillate; leaves lanceolate, serrate, petiolate, hairy ; stamens as long as the corolla.

HAB. Sandy soils. Aug.-Sept. 4. A foot high; fl. pale purp.

3. M. borealis: ascending, pubescent; leaves petiolate, oval-lanceolate, acute at each end; flowers verticillate; stamens exsert.

HAB. Low grounds. July-Sept. 4. Stem $2 \mathrm{ft}$. high, hairy backwards.

\section{ISANTHUS. Labiatce.}

S. cceruleus.

HAB. River shores. July-Aug. ๑. Viscid-pubes., 1 ft. high; leav. oval-lan., acute, 3-nerved; fl. axill., pedunc., blue.

372. HYSSOPUS. Hyssup. Labiatce.

H. nepetoides: spikes verticillate, cylindric ; leaves subcordate, ovate, acuminate, dentate.

HAB. Woods. July. 4. Stem 4-6 ft. high, pubes. ; $f$. yell.-zoh., or pale purp.

373. NEPETA. Catnep. Labiatce.

N. Cataria: flowers spiked; whorls slightly pedunculate; leaves petiolate, cordate, dentate, serrate. HAB. Fields, \&c. June-Sept. 4. Stem 2-3 ft. high; fl. wh. punc. §.

374. LAMIUM. Dead-nettle. Labiate,

L. amplexicaule: Aloral leaves broadly cordate, sessile, amplexicaul, crenate, or ingised ; lower ones petiolate. 
HAB. Cultivated grounds. April-Nov. ๑. Slem 6-8 in. high, suberect; $f$. red. $\S$.

375. GALEOPSIS. Hemp-nettle. Labiatc.

1. G. Tetrahit: stem hispid incrassated between the joints ; leaves ovate, hispid, serrate ; corolla twice as long as the calyx; upper lip nearly straight.

HAB. Waste grounds. July-Aug. $\odot$. Stem 1$2 f t$. high; $f$. verticill., purp. §.

2. G. Ladunum: stem hairy ; internodes not swollen; leaves lanceolate, subserrate, hairy; upper lip of the corolla slightly crenate.

HAB. Waste grounds. July. ○. A foot high; pet. very short; $f$ l. rose-col. $\S$.

376. STACHYS. Woundwort. Labiatce.

1. S. hyssopifolia: scarcely pubescent, erect, slender; leaves sessile, linear-lanceolate, remotely subdentate; whorls about 4 -flowered.

HAB. Meadows. July. 4. Stem 6-10 in. high; leav. sublin.; fl. purp.

2. S. aspera: stem erect, retrorsely hairy; leaves subpetiolate, lanceolate, acutely serrate, whorls about 6 -flowered; calyx teeth divaricate, spiny.

HAB. Fields and wet places July 2\%. A foot high; leav. pubes.; fl. purp.

3. S. sylvatica: leaves shortly petiolate, ovate, subcorrlate, acuminate; whorls 6 -flowered; stem retrorsely pilose-hispid; calyx hispid, with lanceolate, very acute segments.

HAB. Woods. Aug. 4. Stem 1-2 ft. high, erect; leav. hairy, obtusely ser.; fl. purp.

377. LEONURUS. Motherwort. Labiatce.

L. Cardiaca: inferior leaves ovate, 3-lobed; uppermost oncs entire.

HAB. Waste place.. July-Aug. 4. Stem 2-3 ft. high. bran; leav. spread.; fl. vert., red-col.

373. GLECHOMA. Ground-Iry. Labiato.

f. hederacea: leaves reniform, crenate. 
HAB. Hedges, \&c. April-June. 4. Root crecp.; stem decumb.; fl. axill., in threes, blue. $\S$.

379. MARRUBIUM. Horehound. Labiatce.

M. vulgare: stem erect; leaves roundish-ovate, dentate, rugose ; calyx with 10 setaceous, uncinate teeth.

HAB. Road-sides, \&c. Stem 12-18 in. high, whitish pub. ; leav. woolly beneath.

380. PYCNANTHEMUM. Mountain-mint. Labiate. *. Stamens exserted.

1. P. incanum: leaves oblong-ovate, shortly petiolate, hoary-tomentose; heads of flowers pedunculate, compound, lateral and terminal; bracts setaceous.

IIAB. Rocky hills. July-Aug. 4. Plant whitish, soft, 2 ft. high; fl. pale red.

2. P. aristatum: leaves lanceolate-ovate, subserrate, on very short petioles, whitish; heads sessile; bracts awnerl.

IIAB. Dry woods. July-Aug. 4. Stem 1-2 ft. high; upper leav. hoary.

3. P. linifolium: stem straight, much branched, somewhat scabrous; leaves linear 3-nerved, very entire, smooth; heads terminal, in a fasculate corymb.

HAB. Dry swamps. Aug. 4. Stem 12-18 in. fastig. branch; $f$. minute, wh.

**. Stamens included.

4. P. lanceolatum: stem straight, branched, subpabescent ; leaves subsessile, linear-lanceolate ${ }_{2} \mathrm{en}$ tire; heads sessile, fisciculate-corymbed.

HAL. Dry hills. Aug. 4. Stem 2 ft. high; lcav. long, nerved; 月. minute, wh.

j. P. verticillatum: leat es ovate-lanceolate, very en. tire ; whorls sessile, compact ; bracts acuminate. HAB. Mountains. July-Aug. 4 .

6. I'. muticum: leaves ovate-lanceolate, subdentate, smoothish ; heads terminal; bracts lanceolate, rit. ther acute. 
HAB. Rocky hills. July-Aug. \&. Stem 2 fi. high; leav. large; $f$. woh.

\section{CLINOPODIUM. Wild-basil. Labiatce.}

C. vulgare : leaves ovate, subserrate; whorls hairy ; pedicels branched; bracts setaceous.

HAB. Rocky woods. July. 4. Stem 2 ft. high, hairy; leav. peitol. ; fl. purplish.

382. ORIGANUMI. Wild marjoram. Labiatce.

O. vulgare: spikes roundish, panicled, fasciculate, smooth; bracts ovate, longer than the calyx; leaves ovate, entire.

HAB. Fields, \&c. June-Oct. 24. Stems num., $1 \mathrm{ft}$. high; fl. rose-col., crowded.

383. DRACOCEPHALUM. Dragon's head. Labiatce.

1. D. virginicum : spikes elongated, crowded; bracts subulate; teeth of the calyx short, subequal; leaves linear-lanceolate, serrate.

HAB. Mountain meadows. Aug. 4. Stcm 1-2 ft. high, simp., sm. ; fl. spik., purp.

2. D. denticulatum: spikes elongated, with the flowers remote; bracts subulate; teeth of the calyx subequal ; leaves obovate-lanceolate, toothed above. [HAB. Mountains. June. 4. Stenn $1 \mathrm{ft} . \mathrm{high}, \mathrm{sm}$. : leav. subundul.; fl. purp.

384. PRUNELLA. Self-heal. Labiatce.

P. vulgaris: leaves ovate-oblong, petiolate; upper lip of the calyx truncate, 3 -awned; stem ascending. HAB. Meadows. May-Aug. 24. A foot high, hairy; $f l$ in large ovate spikes, purp.

385. SCUTELLARIA. Skull-cap. Labiata:

*. Flowers axillary, solitary.

1. S. galericulata: somewhat branched; leaves cord. ate-lanceolate, subsessile, crenate; flowers axillary, solitary. 
HAB. Marshes. Aug. 4. Stem 12-18 in. high, minutely pubes.; fl. large, blue.

2. S. gracilis: stem mostly simple; leaves remote, broad-ovate, dentate, smooth and sessile, scabrous on the margin; upper ones smaller, entire; flowers axillary.

HAB. Shady rocks. June. 4. Stem 8-12 in. high, slend., erect; leav. veined; $A$. very small.

\section{**. Flowers racemose.}

3. P. lateriflora: much hranched, smoothish; leaves on long petioles, ovate, dentate, sometimes cordate, membranaceous; racemes lateral, leafy.

HAB. Wet meadows. July-Aug. 4. Stem 12 ft. high; racem. long; fl. small, blue.

4. S. integrifolia: stem nearly simple, densely pubescent ; leaves subses sile, oblong, obtuse, cuneate at the base, obscurely toothed; racemes loose, leafy.

HAB. Swamps. June. 4 . Stem $18 \mathrm{in}$ high; $f$. large, blue.-Plant very bitter.

j. S. hyssopifolia: minutely and densely pubescent, branched; leaves lanceolate-linear, very entire; rare.

HAB. Swamps. June. 24. Stem $1 \mathrm{ft}$. high; leav. obtuse; fl. very large.

C. S. ovalifolia: nearly simple, pubescent ; leaves remote, rhombic-ovate, obtuse, attenuated at the base into the petiole, rounded-crenate; racemes terminal, loose ; bracts lanceolate, entire.

HAB. Rocky woods. July. 4. Stem $1 \frac{1}{2}-2 f t$. high ; lower leav. cord.; fl. large.

7. S. cunescens: branched; leaves ovate, acute, petiolate, acutely toothesl, under side, with the bracts and flowers hoary-villose ; lower leaves subcordate; racemes pedicellate, suhpaniculate, axillary and terminal; bracts ovate-lanceolate, longer than the calyx.

HAB. Woods. July. 4. Stem $2-3 \mathrm{ft}$. high; leav. large, whitish beneath; $\Omega$. deep blue. 
386. TRICHOSTEMA. Blue curls. Labiate.

T. dichotoma: leaves rhombic lanceolate, aitenuate at the base, pubescent.

HAB. Dry hills. July-Sept. ○. Plant aromatic.-Siem 6-10 in. high, brach.; fl. blue.

B. linearis: leaves linear, smoothish.

HAB. Sandy fields. Stem viscidly pubescent.

\section{ANGIOSPERMIA.}

\section{PHRYMA.}

P. leptostachya.

HAB. Rocky woods. July-Aug. H. Stem 2$3 \mathrm{ft}$. high ; leav. loose, ovate, dent., petiol.; spikes very long, slend.; fl. purp.; fr. reflex.

388. VERBENA. Vervain. Verbenacec.

*. Leaves laciniate.

1. V. hastata : erect; leaves lanceolate, acuminate, incisely-serrate; inferior ones lobed or subhastate; spikes filiform, erect, panicled ; flowers tetrandrous. HAB. Low grounds. July-Aug. 4. Stem 35 ft. high; leav. rough; fl. purp.

2. V. spuria : stem decumbent, much branched, divaricate ; leaves many-cleft; spikes filiform, loose ; bracts longer than the calyx.

HAB. Sandy fields. Aug. Nov. $\odot$ s. Stem 1-2 ft. long; leav. scab.; fl. pink.

**. Leaves entire.

3. V. urticifolia : erect, subpubescent; leaves ovalacute, serrate, petiolate spikes filiform, loose, axillary and terminal; flowers tetrandrous.

HAB. Fields and road-sides. July-Aug. 24. Stem 2-3ft. high; spik. not imbric.; $f$. wh.

4. V. angustifolia: erect, mostly simple; leaves linear-lanceolate, attenuate at the base, remotely toothed, with elevated vens ; spikes filiform solitary, axillary and terminul. 
HAB. Rocky hills. July. 4. A foot high, hairy; leav. rugose; fl. blue.

\section{ZAPANIA. Verbenacec.}

1. Z. nodiflora: leaves ovate-cuneiform, serrate above ; spikes capitate-conic, solitary, on long peduncles; stem herbaceous, creeping.

HAB. Banks of rivers. July. 4. Stem $6-8$ in, long; pedunc. flif.; head small, blue.

9. Z. lanceolata : leaves linear-lanceolate, acutely serrate; spikes capitate-conic, on elongated peduncles; stem herbaceous, creeping.

HAB. Banks of rivers. July. H. Fl. white. (Blue?)

\section{HERPESTIS. Scrophularice.}

H. cuncifolia: very smooth; leaves cuneate-obo. vate, obscurely crenate bove; peduncles as long as the leaves.

IIAB. Overflowed banks of rivers. Aug. 4. Leav. thick . $f$. minute, pale blue.

\section{LIMOSELLA.}

L. subulata: leaves linear, very narrow, scarcely dilated at the apex; scape 1 -flowered, as long as the leaves.

IIAB. Muddy shores. Aug. 4. An in. high; leav, rad.; fl. minute, bl.-rehite.

\section{SCROPHULARIA. Figwort. Scrophularice.}

1. S. marilandica: leaves cordate, serrate, acute, rounderl at the base ; petioles ciliate below ; panicle fasciculate, loose, few-tlowered ; stem obtuseangled.

IIAB. Woods. July-Oct. 4. Stem 2-4 ft. high, smooth; leav. thin; $f$. gr.-purp.

$\therefore$ S. lanceolata: leaves lancenlate, unequally and incisely serrate, acute at the bise; petioles naked; fascicles of the panicle corymbed.

HAB. Woods. Aug. 4. Stem 3 ft. high, smooth; leav. repand.-scrr. ; $f$. gr.-purp. 
393. BIGNONIA. Trumpet-flower. Bignoniaced.

B. radicans: leaves pinnate; leaflets ovate, dentate, acuminate; corymb terminal ; tube of the corolla three times as long as the calyx ; stem creening.

IIAB. Banks of rivers. July-Aug. $\zeta_{2}$. Stem climbing by radicles; $f$ l. very large, scarl.

\section{RUELLIA.}

R. strepens : erect, hairy ; leaves petiolate, lanceolate-ovate, very entire; peduncles 1 -3-flowered; segments of the calyx lanceolate, hispid, half as long as the tube of the corolla.

HAB. Woods. July. 4. Stem 8-12 in. high; leav. opp.; fl. axill. and term., blue or white.

\section{BUCHNERA.}

B. americana : stem simple; leaves lanceolate, subdentate, rough, 3-nerved; spikes with the flowers remote.

HAB. Sandy woods. Aug. 4. A foot high; lear. opp.; fl. blue.-Plant dries black.

396. ANTIRRHINUM. Toad-flax. Scrophularice.

$$
\text { *. Steins procumbent. }
$$

1. A. Elatine: procumbent, hairy; leaves alternate, hastate, very entire; peduncles solitary, very long.

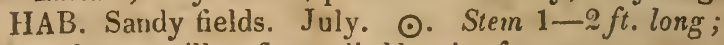
pedunc. axill.; fl. small, bl.-wh. §.

\section{**. Stems erect.}

2. A. Linaria: leaves linear-lanceolate, crowded; spikes terminal ; flowers imbricate; calyx smooth, shorter than the spur.

HAB. Road sides, \&c. June-Nov. 24. Stem 12 ft. high; leav. pale.gr.; fl. yellow.

3. A. canadense: assurgent, smooth, mostly simple; leaves scattered, erect, linear, obtuse; flowers racemose ; scions procumbent. 
HAB. Wet or dry soils. July-Nov. ๑. A foot high, very slend.; $f$. very sm., blue.

\section{COLLINSIA.}

C. verna: leaves ovate-oblong, sessile, obtuse, the inferior ones attenuater into long petioles.

IIAB. Kiver banks. July. ๑. A foot high, mi . nutely pubes.; pedınc. axill.; fl. particoloured.

\section{GERARDIA. \\ *. Flowers purple.}

1. G. purpurca: stem angular, much branched; leaves scabrous linear, long and acute; flowers subses. sile; segments of the calyx subulate.

HAB. Sandy soils and swamps. August-Oct. $\odot$. Stem 1-2 ft. high, slend.; fl. large, axill.

9. G. maritima: stem angular; leaves linear, fleshy, short, rather obtuse ; flowers pedunculate ; uppe: segments of the corolla ciliate; calyx truncate, with minute teeth.

IIAB. Salt marshes. July-Sept. ๑. Stem 6-.. $12 \mathrm{in.} \mathrm{high;} f$. middle-sized.

3. G. tenuifolia: much branched; leaves linear, acute scabrous; peduncles axillary, longer than the flowers; teeth of the calyx acute.

HAB. Fields and woods. Aug.-Sept. ๑. Slem 6-10 in. high ; fl. spread., smooth.

1. G. auriculatu: subsimple, scabrous; leaves ovate, lanceolate, auriculate at the base, very entire. sessile; flowers sessile, nxillary.

"HAB. Fields and mountains. Aug. ๑. Stcm \&--

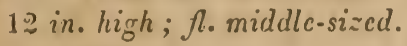

**. Flowers yellow.

5. G. Mara; pubescent; stem nearly simple; learesubsessile, lanceolate, very entire or dentate; the inferior ones incised; flowers subsessile.

HAB. Woods. Aug.-Sept. 4. Stcm 2-3 fi. high. fl. large.

f. (i. glauca: smooth; stem paniculate; leaves pe$\mathrm{X}$ 
tiolate, pinnatifid; upper ones lanceolate; flowers pedicellate.

HAB. Woods. Aug.-Sept. Stem 3-5 ft. high, glauc; leav. pale beneath.

i. G. pedicuslaria: stem paniculate, pubescent ; leaves smoothish, oblong, pinnatifid; segments uncinate, serrate; flowers axillary, pedicellate ; segments of the calyx leafy, incisely dentate.

HAB. Woods and mountains. July-Aug. 4. Stem 2 ft. high, much branch.

399. PEDICULARIS. Lousewort. Scrophularince.

1. P. pallida: stem smooth, branched, with pubescent lines; leaves subopposite, lanceolate; pinnatifidly toothed and crenate, scabrous on the margin; galea of the corolla truncated at the apex; calyx bifid with rounded segments.

HAB. Swamps. Sept. 4. Stem 1-2 ft. high: fl. large, straw-yell. and purp.

2. P. canadensis : stem simple : leaves pinnatifid, incisely toothed; spike leafy at the base, hairy : galea of the corolla with two setaceous teeth; calyx truncate downward.

HAB. Wet meadows. May-July. 4. Stem 8-12 in. high; fl. yell. and purp.

3. P. gladiata: stem simple ; leaves lanceolate, pinnatifid, dentate; spikes leafy, hairy, with the flowers alternate; capsule terminating in a long flat point.

HAB. Wet meadows. May-June. 4. Fl. yell. and purp.-A var. of No. 2?

\section{MIMULUS. Monkey-flower.}

1. M. ringens : erect, smooth; leaves sessile, lanceolate, acuminate; peduncles axillary, longer than the flower.

HAB. Wet meadows. Aug. 4. Stem 2 ft. high, ang; fl. large, pale purp.

9. M. alatus : erect, smooth; leaves petiolate, ovate, acuminate, serrate; peduncles axillary, shorter than the flowers; stem quadrangular, winged.

HAB. Wet meadows. Aug. 4. Stem $2 \mathrm{ft}$. high; teeth of the cal. obl. acum. 
401. CHELONE. Shell-flower.

C. glabra: smooth; leaves opposite, lanceolate oblong, acuminate, serrate; flowers in dense spikes.

HAB. Wet places. Aug.-Oct. 4. Stem $2 \mathrm{ft}$. high, simp.; fl. large, or rose-col.

\section{PENTSTEMON.}

1. P. pubescens: stem pubescent; leaves lanceolateoblong, sessile; repindly serrulate ; flowers paniculale; sterile filament bearded above the middle. HAB. Mountain meadows. June. 4. Stem $1 \frac{1}{2}$ ft. high, slend.; $f$. pale purp.

2. Y. lavigatum: smocth; leaves ovate-oblong, amplexicaul at the base, denticulate; the inferior very entire; flowers paniculate; sterile filament berrded above.

HAB. Low stony grounds. July. 4. Stem 1-2 ft. high ; fl. pale purp.

403. MARTYNIA.

M. proboscidea: stem lecumbent, branched; leaves alternate, cordate, very entire, villous.

HAB. River banks. Aur.-Sept. ○. Stem thick, viscid-pub.; fl. large, wh., punct.

404. SCHWALBEA. Scrophularince.

S. americana.

HAB. Sphagnous swamps. Sept. 4. Stem 1218 in. high, simp.; leav. alt. ob!. entire; $f$. racem., subsess., dark-purp.

405. EUChroMA. Painted-cup. Scrophularince.

E. coccinea : leaves and coloured bracts pinnatifidly 3-cleft ; segments diraricate; calyx 2-cleft, nearly equal with the corolla; segments retuse and emarginate.

HAB. Wet meadows. May-June. 4. Stem 8-1? in. high; bracts scarl; cor. yell. 
406. BARTSIA. Scrophularince.

B. pallida: leaves alternate, linear, undivided; upper ones lanceolate; floral ones subovate, slightly toothed at the end ; all 3-nerved; teeth of the calyx entire.

HAB. High mountains. Aug. 4. Stem pubes.; fl. yell., pubes.

107. MELAMPYRUM. Cow-wheat. Melampyracece.

M. lineare: leaves linear-lanceolate; the floral ones with setaceous teeth at the base; flowers axillary.

IIAB. Woods. June-July. ๑. Stem 8-10 in. ligh, bran.; fl.-leav. ovate-lan.

408. OROBANCHE. Broom-rape. Orobanchece.

1. O: americana: stem very simple; imbricate with oval-lanceolate scales; spike terminal, smooth; corolla recurved, with the stamens exserted.

HAB. Woods. July. 4. Stem 4-6 in. high, very thick, br.-yell., leafless, scaly; ft. bract.

2. O. uniflora: stem very short; peduncles 2 , elongated, scapiform, 1-flowered, naked; scales smooth, concave; lobes of the corolla oblong-oval, with a pubescent coloured margin.

IIAB. Woods. May-July. 4. Pedunc, 4-6 in. long, subpubes.; fl. recurv., bl.-rch.

409. EPIPHAGUS. Beech-drops. Orobanchea.

E. virginiana.

MAB. Beech woods. Sept. 4. Leafless, branched, smooth; 8-12 in. high; $f$. distant ; abort. ones larger, purplish.

\section{TETRADYNAMIA.}

\section{SILICULOSA.}

110. CAKILE. Silicle compressed, of 2 single-seederi joints ; superior joint with an erect single: 
seed; inferior with a pendulous (sometimes abortive) seed.

411. THLASPI. Silicle compressed, emarginate, manyseeded; valves carinate, (often winged) Filam. without teeth, distinct. Cal. equal at the base.

112. DRABA. Silicle entire, oval or oblong; valves flat or convex; cells many-seeded. Seeds not margined ; cotyledons accumbent.* Filam. with. out teeth.

413. ALYSSUM. Silicle orbicular, or elliptical; valves flat, or convex in the centre. Seeds $2-4$ in each cell, compressed, sometimes membranaceously winged; cotyledons accumbent. Cal. equal at the base. Pet. entire. Stam. mostly toothed.

414. LEPIDIUM. Silicle ovate or subcordate; valves carinate, dehiscent; cells 1 -seeded. Cotyledons incumbent.

115. CAMELINA. Silicle subovate, many-seeded; valves turgid. Cotyledons incumbent. Filam. without teeth.

116. SUBULARIA. Silicle oval, many-seeded; valves convex. Stig. sessile. Cotyledons incumbent, linear, biplicate.

\section{SILIQUOSA.}

117. DENTARIA. Silique. lanceolate; valves, Hat, nerreless, often opening elastically ; receptacles not winged. Funiculus dilated. Seeds in a single series, ovate, immarginate; cotyledons accumbent.

118. CARDAMINE. Silique linear, with the margins truncate; valves flat, nerveless, often opening elastically, narrower than the dissepiment. Seed not margined, with a slender funiculus.

419. $\Lambda$ RABIS. Silique linear; valves flat, 1-nerved. Sceds in a single row. Cotyledons accumbent. Cul. erect.

The cotyledons are uccumbent when the back of one of theni is applied to the curved radicle: incumbent when the edges of the cotyleclons are applied to it. 
420. BARBAREA. Silique 4-edged. Cotyledons accumbent. Seeds in a single row. Cal. equal at the base, erect. Shorter filaments with inter" mediate glands.

121. NASTURTIUM. Silique subterete, often short; valves concave, nerveless, not carinate. $\mathrm{Cal}$. equal, spreading. Cotyledons accumbent.

422. SISYMBRIUM. Silique subterete. Cotyledons incumbent, sometimes oblique, flat. Cal. mostly spreading, equal at the base.

423. HESPERIS. Silique subquadrangular, or ancipital. Stig. subsessile, of 2 connivent lobes. Cotyledons incumbent, flat. Cal. closed, sulcate at the base.

424. SINAPIS. Silique subterete, 2-valved; (sometimes of 2 articulations, of which the upper one is nerveless.) Cotyledons conduplicate. Seeds globose, in a single row. Cal. spreading.

425. RAPHANUS. Silique valveless. Cotyledons conduplicate. Cal. closed.

426. CLEOME. Cal. 4-leaved, spreading. Pet. 4, unequal. Stam. 6, unequal, often connected at the base. Silique stipitate in the calyx.

12\%. POLANisia. Cal. 4-leaved, spreading. Pet. 4, unequal. Stam. 8-32. Silique sessile in the calyx.

\section{TETRADYNAMIA.}

\section{SILICULOSA.}

\section{CAKILE. Sea-rocket. Cruciferu.}

C. maritima: $\beta$. americana : leaves cuneate-oblongobtuse, sinuately toothed; joints of the silicle 1 seeded; the uppermost ovate, acute.

HAB. Sea-coast. July-Oct. ๑. Plant flesh! heavy, branch.; $f$. purp., corymb.

\section{THLASPI. Penny-cress. Cruciferce.}

1. T'. arvense: leaves oblong-sagittate, coarsely tooth- 
ed, smooth; silicle suborbicular, longer than the pedicel ; its wings dilated longitudinally.

HAB. Fields. June. $\odot$. Stem erect, 8-12 in. high; leav. smooth; $f$. woh.

2. T. tuberosum: leaves rhombic-ovate, obsoletely dentate, smooth, sessile; radicle ones on long petioles; stem pubescent; root tuberous; silicle orbicular.

HAB. Pennsylvania. April-May. Stem 4-5 in. high; $f$. rather large, rosaceous.

3. T. Bursa-pastoris: silicle triangular-obcordate, without wings ; cells many-seeded; radicle leaves pinnatifid.

HAB. Pastures, \&c. March-Oct. ๑. Stem 612 in. high; fl. small, corymb., wh. $\S$.

412. DRABA. Whitlow-grass. Cruciferc.

$$
\text { *. Petals entire. }
$$

1. D. arabisans: stem leafy, somewhat branched, sulpubescent; leaves lanceolate, acutely dentate; silicle linear, smooth, longer than the pedicel.

HAB. Rocks. §. Silicle elongated, acuminate, contorted.

$$
\text { **. Petals bifid. }
$$

2. D. verna: scapes naked; leaves lanceolate, subincised, hairy ; silicle elliptical-oblong.

HAB. Fields and hills. March-May. ○. leav. all rad.; scape 2-6 in. long; $f l$. wh.

3. D. caroliniana: stem leafy at the base, hispid, taked and smooth at the apex; leaves ovate-roundish, hispid, entire, hispid; silicle linear, smooth, longer than the pedicels.

HAB. Fields. April. O. Stem $1-2$ in. high ; silicle 4-5 in. long; fl. wh.

413. AlySSUM. Gold-of-Pleasure. Crucifera.

1? dentatum: stem erect and herbiceous; raceme: paniculate; radical leaves subruncinately dentate. somerhat scabrous; cauline linear-linceolite, 
sessile, nearly smooth; silicle elliptical, pubescent sborter than the pedicel.

HAB. Rocks. May. 4. Stem 6 in. high; pules. thin; style long, persist.

\section{LEPIDIUM. Pepper-wort. Cruciferce.}

1. L. virginicum: leaves linear-lanceolate, incisely serrate, smooth ; flowers with 2-4 stamens; silicle orbicular, emarginate; stem branched above.

HAB. Sandy fields. June-Oct. $\odot$. A foot high, panic. abowe; fl. minute, wh.

2. L. campestre: cauline leaves sagittate, dentate; silicle ovate, winged, emarginate, scaly-punctate. HAB. Hills. May. ๑. A foot high, erect; leav. pubes.; $f$. wh.

\section{CAMELINA. Cruciferce.}

C. sativa : silicle obovate-pyriform, margined, tipped with the pointed style; leaves subentire, lan ceolate, sagittate.

HAB. Hills. June. ๑. Stem $1 \frac{1}{2} f t$. high, branch.; fl. yell., corymb.

416. SUBULARIA. Owl-wort. Cruciferce.

S. aquatica.

HAB. Margins of ponds. July. ๑. Leav. rad. subul.; scape 1-2 in. high; fl. wh.

\section{SILIQUOSA.}

417. DENTARIA. Toothwort. Cruciferce.

1. D. laciniata: leaves in threes, ternate; leaflets 3parted; segments oblong, unequally and incisely dentate; root moniliform.

HAB. Rocky woods. April-May. Stem 8 in. high; root tub.; ft. purplish.

6. pinnatifida: leaflets suhpinnatifidly laciniate.

2. D. diphylla: stem 2-leaved; leaflets ternate, suborate, unequally and incisely dentate; root dentate. 
IIAB. Woods. May. 4. Stem 6-8 in. high; leafl. large, fl. yellowish.

3. D. heterophylla: stem 2-leaved; leaves ternate, petiolate, leaflets linear, sublanceolate, acute, entire ; radical leaflets ovate-oblong, incisely and coarsely toothed.

HAB. Shady woods. June. 4. Root tub.; stem lozs; leafl. ciliate; fl. purp.

4. D. maxima: leaves numerous, alternate, petiolate; leaflets ternate, suboval, incisely dentate; lateral ones lobed; axils without glands; racemes lateral and terminal.

HAB. Woods. 4. Stem $1 \frac{1}{2} f t$. high; leav. 5-7; leafl. very broad; $f$. purp.

413. CARDAMINE. Lady's smock. Cruciferce.

*. Leaves undivided.

1. C. rhomboidea: leaves ovate-rhomboid, obscurely repand-dentate, smooth; inferior ones on long petioles; root tuberous and tibrous.

HAB. Wet meadows. May-June. 4. Stem 8$10 \mathrm{in}$. high, subpubes.; fl. large, wh.

9. C. rotundifolia: leares suborbicular, nearly entire, smooth, petiolate; stem weak, procumbent; root fibrous.

HAB. High mountains. July. H. Siliques spreading, Slend.; fl. sm., woh.

\section{**. Leaves divided.}

3. C. pennsylvanica: smooth, branched; leaves pinnate, often sublyrate; leaflets roundish-oblong obtuse, angularly toothed; stem erect; petals ob. long-linear.

HAB. Wet places. May. July. 4. Stem. 8$12 \mathrm{in.} \mathrm{high;} \mathrm{tcrm.} \mathrm{leafl.} \mathrm{large;} \mathrm{sil.} \mathrm{erect.}$

ß. grucilis: stem slender, weak; leaves with few and narrow segments.

HAB. Wet woods. July. 4. Stem subdecumb. 4-6 in. long; fl. minute, wh.

1. C. virginica: smooth, erect; leaves pinnate; leaflets lanceolate, subauriculate; stem erect ; siliques long, straight and erect. 
HAB. Moist rocks. May-June. 4. Stem 5-8 in. high; leafl. small, subpubes.; fl. wh.

5. C ? teres: leaves sublyrate-pinnatifid; segments oval-oblong; the terminal ones somewhat 3-lobed; siliques short, erect, terete.

HAB. Wet places. June-July. 4 .

6. C. hirsuta: leaves pinnate, hairy; leaflets of the radical ones roundish, petiolate; those of the upper ones oblong, subsessile; petals oblong; siliques erect.

IAB. Pennsylvania. May. ๑ .

\section{ARABIS. Wall-Cress. Cruciferce.}

1. A. sagittata: leaves subdentate, rough, with the pubescence often branched; radical ones ovate or oblong, attenuated into a petiole ; of the stem lanceolate, sagittate-cordate; pedicels as long as the calyx ; siliques striight and erect.

HAB. Hills. June. $\odot$ \& . Stem $1 \frac{1}{2} f t$. high, simp., straight; $f$. small, wh.

2. A. thaliana: leaves hairy, subdentate; radical ones oblong, petiolate; stem branched, hairy at the base, siliques ascending; pedicels much longer than the calyx.

HAB. Dry hills. April-May. ๑. Stem 2-4 in. high, erect; siliq. slend.

3. A. lyrata: stem and upper leaves smooth and glaucous; radical leaves lyrate-pinnatifid, often pilose ; stem branched at the base ; pedicels spread. ing; silique erect.

HAB. Rocky hills. April-July. đ. Stem 812 in. high; caul. leav. lin.; fl. large.

4. A. levigata: stem leaves linear, oblong, sagittate, smooth ; the lower ones subdentate; radical ones obovate; pedicels erect; siliques very narrow and long, at length pendulous.

HAB. Rocky hills. May. 4. Stem $2 \mathrm{ft}$. high; sil. $2 \frac{1}{2}$ in. long; root nerved.

4. A. canadensis: stem leaves sessile, oblong-lanceolate, narrow at the base, pubescent, pedicles pubescent, reflexed in the fruit ; siliques pendulous, sub-falcate, nerved. 
HAB. Rocky hills. June. 4. Stem $2 \mathrm{ft}$. high; ped. 3 times as long as cal.

420. BARBAREA. Winter-cress. Cruciferce.

B. vulgaris: lower leaves lyrate; terminal lobe roundish; upper ones obovate, dentate.

IIAB. Old fields. June. 4. Stem 1-2 ft. high, smooth, leav. very sm.; fl. yell.

421. NASTURTIUM. Nasturtium. Cruciferce.

1. N. officinale: leaves pinnate; leaflets ovate, subcordate, repand.

HAB. In water. June-July. 4. Stems decumb. term. leafl. large; $f$. wh.

2. N. palustre : leaves lyrate-pinnatifid; lobes confluent, unequally dentate, smooth ; petals as long as the calyx ; siliques short, turgid ; root fusiform. HAB. Wet places. July. ๑. Stem 11 ft. high, fl. yell.; pedicel short.

3. N. amphibium: leaves oblong-lanceolate, pinnatifid or serrate; root fibrous; petals longer than the calyx ; siliques elliptical.

HAB. Wet places. June-July. 4. Stem 1-2 $f t$. high; fl. yell.; sil. subreflex.

122. SISYMBRIUM. Hedge-mustard. Cruciferce.

1. S. officinale: leaves cuncinate, and with the stem, hairy ; siliques subulate, appressed.

HAB. Fields. Jure-Oct. $\odot$. Stem $1-2 \mathrm{ft}$. high, branch.; fl. minute, yell.

2. S. Sophia: leaves bipinnate; segments oblong-linear, incised; petals shorter than the calyx; calyx twice as short as the pelicel.

HAB. Sandy fields. July. ๑. Stem \&-12 in. high ; leav. sm. or pubes. ; $\lambda$. yell.

423. HESPERIS. Dame's violet. Cruciferce.

II ? pinnatifida: leaves acutely serrate; superior ones linceolate; inferior spinnatifid-ly rate ; pedicel, at length longer than the calyx. 
HAB. Woods. July. 4. Stem $1 \mathrm{ft}$. high; $f$. pale purp.; pet. entire.

424. SINAPIS. Mustard. Cruciferce.

S. nigra: siliques smooth and even, subquadrangular, appressed ; inferior leaves lyrate ; upper ones lanceolate, entire, petiolate.

HAB. Waste grounds. June-July. ๑. Stem 2-4 ft. high; upper leav. deflex.; fl. yell.

425. RAPHANUS. Radish. Crucifera.

R. Raphanistrum: silique jointed, 1-celled, striate. 3-3-seeded; style long; leaves simply lyrate.

HAB. Fields. June-July. $\odot$. Stcm $1-2 \mathrm{ft}$. high, hispid; leav. scab:; fl. yell. §.

420. CLEOME. Capparides.

C. pentaphylla: smoothish; leaves quinate; the lower and floral ones ternate; leaflets entire and subserrulate; stamens inserted on the pedicel of the germen.

HAB. Cultivated grounds. July. ๑. Stern $2 f t$. high, viscid; pet. wh., on filif. claws.

\section{POLANISIA. Capparides.}

P. graveolens : riscidly pubescent, leaves ternate ; leaflets elliptical-oblong; stamens $8-12$; :ilique : oblong, attenuate at the base, muricate with a glandular puhescence.

HAB. Sandy shores. June. H. Stem 1 ft. high : Al. racem., red.-roh.-Plant fetid.

\section{MONADELPHIA.}

\section{PENTANDRIA.}

428. PASSIFLORA. Cal. 5-parted, coloured. Pet. 5, inserted upon the calyx. Nect. a filamentous crown. Pepo (berry) pedicellate. 


\section{DECANDRIA.}

499. GERANIUM. Cal. 5-leaved, equal. Pct. 5, equal. Stam. $10 ; 5$ alternate ones longer, with nectariferous glands at the base. Pericarps 5, with long awns, united to elongaterl receptacles, at length separating elastically from the summit to the base: arons smooth internally.

\section{POLYANDRIA.}

130. SIDA. Cal. 5-cleft, simple, often angular. Style many-cleft at the summit. Capsules numerous, arranged circularly, 1-celled, 1-3-seeded.

131. ALTHEA. Cal. double; the exterior 6-9. cleft. Capsules numeruus, 1 -seeded, arranged circularly.

432. MALVA. Cal. double; the exterior mostly 3leaved. Capsules numerous, 1-celled, 1-seeled, arranged circularly.

133. HIBISCUS. Cal. double; the exterior many. leaved. Stig. 5. Caps. 5.celled; cells many seciled.

\section{MONADELPHIA.}

PEN'TANDRIA.

128. P'ASSIFlORA. Passion flower. Passiflora.

1. P. lutea: leaves corlate, 3 -loberl, obtuse, smooth; petioles without glinds; peduncles axillary, by pairs ; petals much longer than the calyx.

HAB. Banks of rivers. June--Aug. H . Climb., slend.; fl. small, gr.-yell.

$\therefore$ P. incarnatre leaves 3-lobed, serrate; lobes oblong, acute; petioles with two glands; involucrum 3-leaved; leaflets lanceolate, glandularly dentate; rays of the nectary longer than the corollia.

IIAB. Banks of rivers. Sept. If . Stem long, climh, , pet. white; nect. purp. 


\section{DECANDRIA.}

\section{GERANIUIT. Geraniacece.}

1. G. maculatum : stem angular, erect, retrorsely pubescent, dichotomous; leaves 3-5-parted, incised; radical ones on long petioles; upper ones opposite, sessile; petals entire; filaments scarcely ciliate at the base.

HAB. Woods. May-June. 4. Stem 1-2 ft. high; leaves hairy; fl. large, purp.

2. G. carolinianum: diffuse, pubescent; leaves 5parted; segments 3-cleft; incised peduncles crowded, subfasciculate; petals emarginate, as long as the awned calyx ; pericarps hairy ; seeds smooth.

HAB. Hills and fields. May-June. ๑. Stem 1218 in. long, much bran.; fl. small, wh.

3. G. pusillum: leaves subreniform, 7-lobed; lobes 3-cleft ; petals subemarginate, as long as the pointless calyx; pericarps pubescent, not rugose ; seeds smooth.

HAB. Pennsylrania. May. $\odot$.

1. G. dissectum: diffuse, pubescent ; leaves 5-parted ; segments 3-cleft, linear; petals emarginate, as long as the awned calyx; pericarps hairy ; seeds reticulate.

IIAB. Dry hills. June-July. ๑. Stem $1 \mathrm{ft} . \mathrm{high}$, pubes.; fl. small, pale red.

$\therefore$ G. Robertianum: leaves 3-5-parted; segments pinnatifidly 3-cleft; petals entire, much longer than the angular awned calyx; pericarps small, reticulate, rugose ; seeds smonth.

HAB. Rocky places. May-Sept. $\odot$. Stcm long, red; l. small, pale purp.

\section{POLYANDRIA.}

430. SIDA. Indian Mallow. Malvaces.

1. S. spinosa: leaves ovate, lanceolate, dentate, with the tubercles at the base spiny ; pedicels axillary, 
solitary, shorter than the stipules and petioles; capsules 5 , birostrate.

HAB. Pine barrens. July-Aug. ๑ . Stem 1-? ft. high, lranch.; fl. yell.

$\therefore$ S. Nipcea: leaves palmately 5 -lobed, smooth; lobes oblong, acuminate, dentate; peduncles manyflowered; capsules 10, pointless, acuminate.

HAB. Shady rocky places. July. 4. Stem 3-1 ft. high; fl. small zoh.

3. S. dioica: leaves palmately 7-lobed, scabrous ; lobes lanceolate, incisely dentate; peduncle: many-flowered, bracteate, subcorymbed ; flowers diøcious ; capsules 10 , pointless.

HAB. Stony ground. Oct. 4. Stem 4-5 ft. high. branch.; fl. small reh.

4. S. Abutilon: leaves roundish-cordate, acuminate, dentate, tomentose; peduncle shorter than the petioles; capsules 15 , trur, cite, birostrate, hairy. IIAB. Waste places. July-Aug. ๑. Stem 2-5 ft. high; leav. large; fl. orange. §

431. ALTHAA. Marsh Mallow. Malvacex.

A. officinalis: leaves soft-tomentose, cordate and orate, dentate, entire or somewhat 3-lobed; peduncles axillary, many-flowered, much shorter than the leaves.

HAB. Borders of salt marshes. Aug.-Sept. 4 Stern $2 f t$. high ; fl. large, purp. $\S$

432. MaLVA. Mallow. Malvacea.

I. rotundifolia: stem somewhat prostrate; leave: roundish, cordate, obtusely 5 -Inbed; fructiferous pedicels bent downward, as well as the petioles, pubescent; corolla twice the length of the calyx. IIAB. Waste places. June-Oct. H. Root fusif., pedunc. axill.; fl. small pinli.

\section{HIBISCUS. Malvacer.}

1. H. Moscheutos: leaves ovate, acuminate, serrate. tomentose beneath; petioles bearing the pedun cles; calys tomentose ; capsule sinooth. 
HAB. Brackish marshes. Aug. 4. Stem 3-5fi. high; fl. large, wh., with a crim. centre.

2. H. palustris: leaves ovate, dentate, somewhat 3 lobed, whitish-tomentose beneath, pedicels axillary, distinct from the petioles, articulate above the middle.

HAB. Borders of salt marshes. Aug. 4. $F i$. large, entirely red.

3. H. militaris: leaves 3-lobed-hastate, acuminate, serrate, smuoth ; pedicels articulate in the middle ; corolla subcampanulate; capsule ovate, acuminate, smooth ; seeds silky.

HAB. River banks. July. 4. Stem $3 \mathrm{ft}$. high ; A. solit., axill., rose-col.

4. 11. virginicus: leares acuminate, unequally dentate, subrillose; inferior ones entire, cordate; superior ones ovate cordate, 3-lobed; pedicels longer than the petioles; flowers cernuous; pistils nodding.

IHAB. Borders of salt marshes. Aug. H. Stcm. $3 \mathrm{ft}$. high ; fl. sm., purple.

\section{IIADELPHIA.}

\section{IEXANDRIA.}

434. CORY DALIS. Cal. 2-leaved. Cor. 4-petalled. Filam. in 2 sets. Silique, 2 -valved compressed, oblong, many-seeded.

135. FUMARIA. Cal.2-leaved. Cor. 4-petalled, onc of the petals gibbous at the base. Silicle ovate. or globose, 1-seeded, not opening.

\section{OC'TANDRIA.}

130. POLYGALA. Cal. 5-leaved, persistent; 2 of the leaflets wingshaped and coloured. Caps. obcordate, 2-celled, 2-valved. Seeds pubesGent. 


\section{DECANDRIA.}

* Stamens all connected, (monadelphous.)

437. AMORPHA. Cal. subcampanulate, 5-cleft. Cor. with the vexillum ovate, concave; wings and keel wanting. Legume 1-2-seeded, falcate.

438. LUPINUS. Cal. bilabiate. Anth. alternately oblong and roundish. Legume coriaceous, torulose.

139. CROTALARIA. Vexillum large and cordate; keel acuminate. Filam. united, with a dorsal fissure. Legume pedicellate, turged.

110. GENISTA. Cal. bilabiate; upper lip with 2. lower with 3, teeth. Vexillum reflexed.

* Stamens diadelphous.

† Stigma pubescent.

141. PISUM. Cal. with the segments leafy, equal. Vexillum with 2 protruding plaits. Style compressed, carinate, villous on the upper side. Suture of the legume naked.

142. JA'THYRUS. Style flat, villous on the upper side, dilated upward. Cal. with the 2 upper segments shortest.

443. VICIA. Style transversely bearded beneath the stigma. Cal. with the 3 inferior segmentstraight and longer. Vexillum emarginate.

44. LRVUM. Stig. capitate, every where pubescent.

$\dagger \dagger$ Stigrna smooth.

115. ASTRAGALUS. Keel obtuse. Legume more or less completely 2-celled; inferior suture in flexed.

It Legume mostly 1-seeded. (Not of the preceding sections.)

116. MELILOTUS. Flowers racemose. Cal. tubu lar, 5-toothed. Keel simple, shorter than the wings and vexillum. Legume longer than the calyx, rugose.

117. TRIFOLIÜM. Flowers subcapitate. Legume covered by the calyx, without valves, $2-4$. seeded. 
148. STYLOSANTHES. Cal. tubular, very long bearing the corolla. Germen beneath the corolla. Loment 1-2-jointed, hooked.

449. LESPEDEZA. Cal. 5-parted; segments nearly equal. Keel transversely obtuse. Loment len. ticular, unarmed, 1-seeded.

$f \nmid \uparrow+$ Legume mostly articulate. (Not of the preceding sections.)

150. HEDYSARUM. Cal.5-cleft. Keel transversely obtuse. Loment of several 1 -seeded joints; joints truncate, compressed, mostly hispid.

651. ASCHYNOMENE. Cal. bilabiate. Loment compressed; one of the sutures straight, the other lobed; joints truncate, 1-seeded. Stam. divided into 2 equal sets.

$\dagger+\dagger \dagger+$ Legume 1-celled, many-seeded. (Not of the pre ceding sections.)

152. PHASEOLUS. Keel, with the stamens and style spirally twisted. Legume compressed, falcate. Seeds compressed, reniform.

153. STROPHOSTYLES. Keel, with the stamens and style spirally twisted. Legume terete, somewhat 2-celled. Seeds reniform, nearly cylindrical.

454. APIOS. Cal. subbilabiate, truncate, 1-toothed. Keel falcate, reflexing the apex of the vexillum. Germen sheathed at the base. ' Legume coriaceous, many-seeded:

455. AMPHICARPA. Cal. 1-toothed. Pet. oblong: vexillum with the sides appressed. Stig. capitate. Legume compressed, stipitate, 2-4. seeded.

156. GALAC'TIA. Cal. 4 -toothed, with 2 bracts at the base. Pet. all oblong; vexillum broad, incum. bent. Anth. oblong. Stig. obtuse. Germen on a naked stipe. Legume terete, many-seeded.

15\%. CLITORIA. Cal. tubular or campanulate, 5toothed. Cor. resupinate; vexillum large, expanding, covering the wings. Liegume linear: acuminate, many-seeded:

458. ROBINIA. Cal. campanulate, 4-cleft; upper segment :-cleft.. Fexillum roundish, expandest 
and reflexed. Legume compressed, elongated, many-seeded. Seeds compressed, small.

159. TEPHROSIA. Teeth of the calyx subulate, nearly equal. Stam. monadelphous. Legume compressed, coriaceous.

160. MEDICAGO. Keel of the cor. deflexed from the vexillum. Legume compressed, spiral.

\section{DIADELPHIÃ.}

\section{HEXANDRIA.}

434. CORYDALIS. Fumariacea.

* Corolla with 1 spur at the base.

1. C. glauca: stem erect, branched; leaves glaucous decompound; segments cuneate, 3-cleft; bract= linear, shorter than the pedicels; siliques linear. HAB. Rocks. May-Aug. ○. đ. Stem 8-18 in. high; rool fusif.; $f$. red and yell.

2. C. aurea: stem branched, diffuse ; leares glaucous, doubly pinnate; lobes oblong-linear; bracts lanceolate; siliques linear, 4 times as long as the pedicel.

HAB. Mountains. May. ๑) Stem 8-12 in. long ; fl. small yell.

** Corolla with 2 spurs at the base.

3. C. Cucullaria: spurs straight, divaricate, acute; scape naked; raceme simple.

HAB. Sharly rocks. Apr.-Miay. 4. Root bulb.; leav. 2, decomp.; fl. large, wh.

16 C. furmosa: spurs slightly curved, obtuse ; raceme naked, somewhat compound; stigma 2-angled.

HAB. Shady rocks. May. 4. Root bulb. scape and pedunc. red; $f$. large, rose-col.

$$
\text { ** Petals united, spor:gy. }
$$

5. C. fungosa : stem climbing; leaves furnished with tendrils; racemes axillary, corymbose, nodding: corolla bigibbous at the base.

HAB. Rocky hills. July-Aug. \&. Stem long; lear', decomp.; fl. num.,flesh-col. 


\section{OCTANDRLA.}

436. POLYGALA. Milk-wort. Polygalea.

1. P. vulgaris: leaves linear-lanceolate, rather obtuse stem ascending; calycine wings ovate, obtuse, a little longer than the capsule; flowers in a terminal raceme.

HAB. River banks. 4. Stems num.; fl. blue. cristate; fructiferous cal. green. +

Q. P. scnega: stems numerous, erect, smooth, simple: leaves alternate, ovate-lanceolate; upper ones acuminate; racemes spiked ; calycine wings orbicular ; capsule elliptical, emarginate.

HAB. Woods. June-July. 4. Stems $1 \mathrm{ft}$.higi ; leav.pale gr.; $f$. wh.

3. P. paucifolia: stem simple, erect, naked below; leaves ovate ; terminal flowers, large, cristate; radical flowers apterous.

HAB. Woods and swamps. May-June. 4. Stem 3-4 in. high; leav. acute; fl. 3-4, purp.

1. P. polygama: stems numerous; leaves linear-oblong; attenuate downwards; racemes terminal and lateral, elongated; flowers sessile; radical racemes procumbent, with apterous flowers.

HAB. Fields. June-July. 4'. Stems 4-8 in. high, angular; $f$. purp.

5. P. lutea: stem simple or branched; lower leave: spathulate; upper ones lanceolate; spike subcapitate, obtuse ; calycine wings ovate, mucronate: bracts shorter than the flowers.

IIAB. Swamps in pine barrens. June-Oct. \& . Fl. deep yell.; heads oblong.

ij. P. purpurea: stem fastigiately branched; leaves alternate, oblong-linear ; spikes cylindrical-oblong; flowers beardless; calycine wings cordate-ovate, twice as long as the capsule.

IIAB. Wet pine barrens. July-Aug. ๑ Stem 12-18 in. high; fl. subimbric., rose-col.

$\because$ P. sanguinea: stem fastigiately branched; leaves alternate, narrow-linear; spikes oblong; Howers: 
beardless; calycine wings obovate, as long as the capsule.

HAB. Wet meadows. July-Oct. ๑. Stem 8-

12 in. high; fl. loose, dark rose-col.

3. P. verticillata: leaves verticillate, linear, whorls remote; racemes spiked, acute, pedunculate; flowers cristate; calycine wings roundish; stem erect, branched.

HAB. Dry hills. July-Oct. ๑. Stem very slend., quadrang.; fl. small, greenish-zeh.

3. P. ambigua: leaves linear; the losvest ones verticillate; the rest scattered; spikes acute on very long peduncles; flowers cristate; calycine wings round and veined, as long as the fruit; stem erect. virgately branched.

HAB. Wet woods. ๑. Fl. purplish, distincily pedicellate.

10. P. fastigiata: stem slender, fastigiate; leaves alternate, linear, acute; spikes subcapitate, pellunculate; flowers subcristate; calycine wings spreading, ovate, acute, scarcely larger than the capsule.

HAB. New-Jersey. $\odot$.

11. P. cruciata: stem fastigiate, winged at the angles; leares verticillate in fours, linear-oblong, punctate; spikes dense, sessile; flowers subcristate; calycine wings cordate, cuspidate.

HAB. Open swamps. Aug.-Sept. ๑. Stem often spreading; fl. greenish-purp.

12. P. Urevifolia: stem erect, subfastigiate, winged at the angles; leaves verticillate in fours, oblonglinear, short, sprinkled with resinous dots; spikes pedunculate. partly capitate; flowers subcristate; calycine wings cordate-orate, acute scarcely longer than the capsule.

HAP. Sandy swamps. July-Aug. ๑. Slender; branch. erecl; $A$. red.

13. P. corymbosa: stem simple, angular, corymbose at the summit, many-headed ; radical leaves spathulate-obovate; cauline subequal, linear; calycine wings oblong, cuspidate.

HAB. Sphagnous swamps. July-Aug. Stern 1 ? in. high; fl. yell., dark green when dry. 


\section{DECANDRIA.}

43\%. AMORPHA. Bastard indigo. Leguminosce.

A. fruticosa $\beta$. emarginuta: smooth, subarborescent; leaves petiolate, emarginate ; spikes aggregated, long; calyx hoary, pedicellate, one of the teeth acuminate, the rest obtuse; legume fewseeded.

HAB. New-Jersey? A shrub with spikes of purp. flowers.

\section{LUPINUS. Lupine. Leguminosce.}

L. perennis: perennial; root creeping; stem and leaves smoothish; leaves digitate; leaflets $8-9$, obcuneate, lanceolate, obtuse; flowers alternate; calyx without appendages; upper lip emarginate; the lower entire.

HAB. Sandy woods and hills. May-June. Stem ascend., $12 \mathrm{in.} \mathrm{high;} \mathrm{leav.} \mathrm{digit.;} \mathrm{f.} \mathrm{blue.}$

433. CROTALARIA. Rattle-box. Leguminosce.

1. C. sagittalis: hairy, erect, branching; leaves simple, ovate-lanceolate; stipules lancenlate, acuminate, decurrent; racemes opposite the leaves, about 3-flowered; corolla smaller than the calyx. HAB. Sandy fields and woods. July-Aug. ๑. Stem 12 in. high; Al. yell.; legum. infl., black.

@. C. parviflora: hairy, erect, branched; leaves simple, linear-lanceolate; upper stipules decurrent, with 2 very short teeth; racemes opposite the leaves; corolla smaller than the calyx.

HAB. Sandy fields. June-July. ๑. Leares : -3 in. long; fl. yell.

\section{GENISTA. Broom. Leguminose.}

G. tincloria; leaves lanceolate, smooth; branches terete, striate, erect, unarmed; flowers in a leafy raceme; legume smooth.

HAB. Dry hills. July. Suffruticose, $1 \mathrm{ft}$. high. branches num.; fl. yell., subsess. §. 
441. PISUM. Pea. Leguminosce.

P. maritimum: stem quadrangular, compressed; petioles flat above ; stipules sagittate; leaflets numerous, subalternate, obovate; peduncles longer than the leaves, many-flowered.

HAB. Sandy shores. May-July: 24. Plant pale green; stem decumb.; fl. blue; leg. hairy.

\section{LATHYRUS. Vetchling. Leguminosce.}

1. L. palustris : stem quadrangular, winged; stipules semisagittate, large, ovate, mucronate; leaflets in 3 pairs, oblong-ovate, mucronate; peduncles 4-6-flowered, rather longer than the leaves.

HAB. Wet thickets. June-July. 4. Stem weak; leav. broad, or narrorv-ovate; fl. purp.

2. L. myrtifolius: stem naked, quadrangular; stipules semisagittate, lanceolate, acuminate; leaflets 4 , oblong-lanceolate, acute, mucronate, reticulately veined; peduncles longer than the leaves, about 3-flowered.

HAB. Salt marshes. July-Aug. 4. Fl. purp. and rose-coloured.

3. L. venosus: stem naked, quadrangular; stipules semisagittate, ovate, acuminate; leaflets numerous, (about 5 pairs,) subalternate, ovate, obtuse, mucronate, veined; peduncles shorter than the leaves ; 5-10-flowered.

HAB. Mountains. July. H. Leaflets large; fl. purp.

413. VICIA. Vetch. J.eguminoso.

1. V. satiza : flowers mostly by pairs, sessile; legumes erect; lower leaves retuse; stipules toothed, or laciniate, with : rlark spot beneath.

HAB. Rocky shore. June. $\odot$. Stem $1-2 \mathrm{ft}$. long; leav. pubes. or smooth; fl. sm., blue.

$\therefore$ V. americana: peduncles 4 -10-1lowered, shorter thin the leaves; stipules semisagittate, dentate; leaflets 8-12, elliptical-linceolate, obtuse, smooth.

HAB. Shady thickets. Junc. 4. Stem long; lea: distant; fl. large, purp. 
3. V. Cracca: spikes many-tlowered, retrorsely imbricate, longer than the leaves; leaflets lanceolate, pubescent; stipules semisagittate, mostly entire.

HAB. Meadows and thickets. Aug. 4. Stem subpubes.; leav. pinnate; fl. sm., purp.

4. V. caroliniana: peduncles many-flowered, as long as or longer than the leaves; flowers distant; stipules ovate-lanceolate, entire; leaflets $3-10$, elliptical-lanceolate, rather smooth.

HAB. Rocky hills. May-June. 4. Stem long, climb. ; f. small, num., white.

\section{ERVUM. Tare. Leguminosce.}

1. E. hirsutum: peduncles many-flowered; legumes hairy, :-seeded; leaflets linear-oblong, truncate. HAB. Rocky places, near salt water. May-June. ○. Fl. minute, blue.

2. E. tetraspermum: peduncles mostly 2-flowered; legume smooth, $4-5$-seeded; leaves oblong, truncate.

HAB. With the preceding. May-June. ๑. Stem very slend.; fl. minute, blue.

445. ASTRAGALUS. Milk-vetch. Leguminosa.

1. A. carolinianus: caulescent, erect; leaflets (41) ob). long, pubescent beneath; spikes pedunculate: bracts lanceolate, as long as the peduncles; legumes ovate, tumid, rostrate.

HAB. Mountains. June-July. 4. Spikes dense; A. num., pale yellnw.

2. A. canadensis: caulescent, diffuse; leaflets (21) smooth on both sides; legume subcylindrical, mu. cronate.

IIAB. Barren fields. June. 4. Stem $2 \mathrm{ft.high}$; legume ovate, tumid?; fl. yell.

446. MELILOTUS. Melilot. Leguminosk.

II. officinalis: legumes naked, 2 -seeded, rugose; leaflets ovate-oblong, dentate; stern erect.

HAB. Wet meadows. Aug. ๑. o. Stem 2-3 fi. high; f. yell. in long racem.-Plant odor. 
447. TRIFOLIUM. Trefoil. Leguminosa.

* Legumes 3-8-seeded.

1. T. repens: heads umbellate; legumes 4 -seeded, covered by the persistent corolla; stem creeping.

HAB. Meadows, sc. May-Oct. 4. Stem 612 in. long; pet. very long; $f$. wh.

ค. T. reflexum: decumbent, pubescent; leaflets obovate; stipules obliquely cordate; heads manyflowered; flowers pedicellate, all at length reflexed; legumes mostly 4 -seeded.

HAB. Rocky upland. June. 4. Very pubes.; fl. in large heads, red.

$$
\text { * * Legume 1-sceded. }
$$

$\dagger$ Vexillum deciduous; (Flowers not yellow.)

3. T. pratense: hearls dense, ovate; lower tooth of the calyx shorter than the tube of the monopetalous corolla; leaflets oval, entire; stem ascend. ing.

HAB. Mearows, \&c. May-Oct. 4. Stem 1ft. high; fl. red, frag. \$.

4. T. pennsylvanicum: ascending; stem much branched; flexuous; leaflets ovarn-elliptical, obtuse, very entire; stipules awned; spikes ovate-cylindrical, solitary, dense; lower tooth of the caly $\mathbf{x}$ shorter than the monopetalous corolla.

HAB. Woods and fields. June-Sept. 4. Fl. red. 5. T. arvense : hearls very hairy, oblong-cylindrical; teeth of the calyx setaceous, longer than the $\mathrm{co}^{-}$ rolla; leaflets villous, narrow-obovate.

HAB. Dry fields. June-Sept. ๑. Stem 6-12 in high; $f$. minute, wh. or pink.

it Vexillum persistent, scarious. (Flowers yellow.)

¿. T. procumbens: heads oval, densely imbricate; vexillum sulcate, reflexed; stem procumbent; middle leaflet petiolate. 
HAB. Sandy soils. June. ๑. Stem 2-8 in. long; leafl. obovate; $f$. brown when old.

7. T. agrarium: erect, subpubescent; leaflets lan. ceolate-cuneate, obtuse ; intermediate one sessile; stipules lanceolate, acute ; heads oval, imbricate ; vexillum deflexed, persistent ; teeth of the calys subulate, smoeth, unequal.

HAB. Sandy soils. June. ๑. Slem $1 \mathrm{ft}$. high; $f$. small.

8. T. campestre: spike orate, imbricate; vexillum deflexed, persistent; leaflets lanceolate-ovate, middle one petiolate; stem sub-diffuse; branches decumbent.

HAB. Sandy soils. June. ๑.

448. STYLOSANTHES. Leguminosa.

S. elatior : stem erect, pubescent on one side; leaflets lanceolate, smonth, acute ; bracts lanceolate, ciliate, 3-4-flowered; lomerit inlurated, 1 -seeded.

HAB. Sandy woods. July-Aug. 4. Stem 12 ir. high, branch.; bracts hisp.; fl. yell.

449. LESPEDEZA. Bush-clover. Leguminose.

1. L. frutescens: stem erect; leaflets elliptical, obtuse, silky-pubescent; flowers in subcapitate fascicles, shorter than the leaves, conglomerate towards the summit of the stem; loments hairy, shorter than the villous calyx.

HAB. Dry woods. Sept. 4. Stem 2-3ft. high, hairy; petiol. short; cor. wh. and red.

9. L. angustifolia: stem erect ; leaflets linear-lanceolate, hoary, pubescent ; racemes capitate, longer than the leaves; corolla longer than the calyx.

HAB. Sandy woods. Sept. 4. Stem 2-3 $\mathrm{ft}$. high; leav. 2-3 lin. broad; heads sm.; fl. wh.

3. L. Stuvei: stem erect, simple, silky-villous; leaflets elliptic-oval; racemes pedunculate scarcely longer than the leaves, loose; loments naked, pubescent.

IIAB. Sandy fields. 4. Stem 2-3 ft. high, pisbes. soft; pedunc. 1 in. long; cor. purp. 
4. L. hirta: erect, branched, very villous; leaflets roundish ; racemes capitate, axillary, on peduncles longer than the leaves; corolli and loments as long as the calyx.

HAB. Dry woods. Aug.-Sept. 4. Stem 2-4 ft. high; racem. ovate, dense; $f$. reddish-wh.

כ. L. sessiliflora : erect, somewhat branched; leaflets oblong-oval, obtuse ; fascicles of flowers subsessile; axillary ones partly racemose; loment naked, acute.

HAB. Dry woods. Aug.-Sept. 4. Stem $2 f t$. high, slend.; pet. long; $f$. violet.

6 . L. reticulata : stem erect, simple or branched, nearly smooth; leaflets oblong-linear, hairy beneath; fascicles of flowers subsessile, numerous ; axillary ones sub-racemose; loment ovate, reticulate, acute.

HAB. Dry woods. Aug. 4. Stem $2 \mathrm{ft}$. high, slend.; leaves 2-lin. broad; $f$. violet.

7. L. violacea: diffuse, much branched; leaves on long petioles; leaflets nval-elliptic, obtuse, substrigose beneath; racemes setaceous, longer than the petioles, sub-umbellate; flowers by pairs, distinctly pedicellate; loments rhomboidal, reticulate, and smooth.

HAB. Dry,woods. Aug. 4. Stems long, slend., nearly procumb.; fl. violet.

3. L. procumbens : slender, procumbent, every where pubescent; leaflets oval; perluncles very long. setaceous ; racemes short; loments suborbicular; pubescent.

IIAB. Sandy fields and woods. Aug.-Sept. 4. Stems 2-3 ft. long; $f$. purp.

9. L. prostrata: smooth, prostrate; leaves on very short petioles; leaflets obovate-elliptic, obtuse ; racemes axillary and terminal, subpiniculate; peduncles very long; loments oval, subpubescent. HAB. Sandy soils. Aug. 4. Stem very slend. branch.; $f$. violet.

450. HEDYSARUM. Sainfoin. Leguminosa.

1. H. canadense: leaves ternate, oblong-lanccolate ; 
stipules filiform; flowers racemed; joints of the loment obtusely triangular, hispid.

HAB. Woods. July. 4. Stem 3 ft. high; lear. 3 in. long ; fl. purp.; bract. long.

1. H. canescens : erect, hairy ; stem with ciliate angles, hispid; leaves ternate, roundish, with appressed hairs beneath; stipules ovate, acuminate ; racemes paniculate; bracts cordate; joints of the loment triangular, hispid.

HAB. Dry barren woods. June-Aug. 4. Fl. pale purp.

3. H. marilandicum : erect, branched, hairy ; leaves. ternate, oblong, subvillous beneath; stipules subulate; racemes paniculate; loments 3 -jointed; joints rhomboidal. reticulate, a little hairy.

HAB. Dry woods. July-Aug. 4. Stem hairy above; petiole long; pan. loose.

4. H. ciliare: erect, somewbat hairy ; leaves ternate, approximate, on very short petioles; leaflets small, oval, obtuse; stipules subulate; panıcle terminal; joints of the loment $(2-3)$ semiorbicular, hispid.

HAB. Dry swamps. Aug. 4. Stem $2 \mathrm{ft}$. high, slend.; leav. small, numerous.

3. H. obtusum: stem erect, very slender, smoothish ; leaves ternate, ovate, obtuse, subcordate at the base ; stipules subulate; panicle terminal ; joints of the loment semiorbicular, reticulate, hispid.

HAB. Wonds. Ang. 4. Stem 2 ft. high, branch. : leav. 1 in. long; lom. 3-joint.

6. H. viridiflorum: stem erect, branched, scabrous; leaves ternate, ovate, obtuse, scabrous abnve, villous and very soft beneath; panicle terminal, very long, naked; joints of the loment triangular.

HAB. Woods: Aug. 4. Stem 3 fl. high, pubes.; fl. greenish externally.

7. H. humifusum: stem prostrate, smooth; leaves ternate; leaflets ternate, slightly hairy ; racemes elongated; joints of the loment subrhomboidal.

HAB. Wnods. Aug. 4. Leav. subacute; pan. loose; joints $4-5$, hisp.

3. H. rotundifolium: stem prostrate, hairy ; leaves ternate, suborbicular, hairy; stipules cordate. 
reflexed; racemes axillary, paniculàte ; joints of the loment subrhomboidal.

HAB. Rocky woods. Aug. 4. Stem 2-3 ft. long; leav. large ; racem. fere-fl.

9. H. paniculatum: erect, smooth; leaves ternate, oblong-lanceolate, or elliptical, smooth; stipules subulate; joints of the loment (4-5) rhomboidal.

HAB. Woods. $\Lambda$ ug. 4. Stem $3 \mathrm{ft}$. high, slend., striate; leav. long; loment large.

10. H. strictum : erect, subpubescent ; leaves ternate ; leatlets sublinear, smooth, reticulate; stipules subulate; racemes axillary and terminal ; loments mostly 2-jointed; joints lunate-triangular, hispid.

HAB. Pine barrens. Aug. 4. Stem slender, branch.; leav. 2-3 lin. troad.

11. H. laevigatum: very smooth; stem erect, or subdecumbent; leaves ternate, on long petioles, ovate, acute, subcoriaceous, somewhat glancous ; panicle terminal; bracts ovate, acute, shorter than the flower buds; joints of the loment triangular.

HAB. Woods, Aug. 4. Petiol. long; lear. large; loment 3-5-joint, hisp.

12. H. nudiflorum : leaves ternate, broad oval, acuminate, slightly glaucous beneath; scape panicled, smooth, radical; joints of the loment obtusely triangular.

HAB. Woods. Aug. 4. Stem 8-10 in. high; pet. long; scape 2-3 fi. long, slend.

13. H. acuminatum : erect, simple, pubescent; leaves ternate, ovate, conspicuously acuminate, a little hairy ; panicle terminal, on a very long naked peduncle; joints of the loment roundish.

HAB. Woods. July-Aug. 4. Stem 8-12 in。 high; pan. 1-2 fi. long.

14. H. cuspidatum: erect, smooth; leaves ternate, ovate, conspicuously acuminate; stipules lanceolate; panicle terminal; bracts large, ovate, acuminate; joints of the loment subtriangular.

$\mathrm{HAB}$. Woods. Aug. 4. Stem 3-5 fl. high; leaves and $f$. large; loment $3-6$ jointed.

15. H. alpinum $\beta$ : erect; leaves pinnate; leaflets Z 2 
oval-oblong, hairy; stipules partly sheathing ; joints nearly round, smooth.

IIAB. Mountains. 4. Fl. purp.

\section{ESCHYNOMENE. Leguminosœ.}

E. hispida: stem erect, and with the petioles, peduncles, and loments hispid; leaves in many pairs : leaflets linear, obtuse; racemes simple, few. flowered.

IIAB. River marshes. Aug. ○. Stem $2-3 f t$. high; leafl. very num. ; $f$. yell. and red.

452. PHASEOLUS. Kridney-bean. Leguminosu.

P. perennis : twining, pubescent ; racemes paniculate, mostly by pairs; leaflets ovate, acuminate, triply nerved; legumes pendulous.

IIAB. Dry woods. July. 4. Stem long; pan. 6-12 in. long; leg. broad-falcate.

453. STROPHOSTYLES. Leguminosa.

1. S. angulosa: leaves ternate; leaflets angular, 23-lobed; flowers capitate, on peduncles longer than the leaves.

HAB. Rocky banks. Aug. ○. Stem prostrate, somewhat hairy; lower leaves entire.

3. S. helvolus : climbing or prostrate ; leaves ternate : leaflets oblong-ovate; flowers capitate, on very long peduncles.

HAB. Sandy fields. July-Aug. 4. Stem branch.; pedunc. $6-8 \mathrm{in}$. long.

454. APIOS. Leguminosa.

1. tuberosa.

HAB. Sharly thickets. July-Aug. 4. Root tub.; stem twining; leaves pirnate; leafl. $5-7$; racem. axill.; fl. dark purp.

\section{AMPHICARPA. Leguminosœ.}

Ł. monoica: leaves ternate, ovate, smooth ; stem hairy : racemes of the stem pendulous, petaliferous, ste- 
rile; radical peduncles, bearing apetalous fertile flowers.

HAB. Woods and thickets. July-Aug. 4. Stem twin., slend.; fl. racem., pale purp.

\section{GALACTIA. Leguminosce.}

1. G. glabella: leaves ternate, ovate, and elliptic, sub. coriaceous, shining, emarginate at each end, smooth above; racemes perlunculate, as long as the leaves; calyx smooth ; legumes pubescent.

HAB. Pine barrens. Aug. 4. Stem prost.; $f$. reddish-purp., large.

9. G. mollis: plant villous-hoary; leaves ternate, oval or elliptic, pale beneath; racemes axillary, simple, elongated, few-flowered; legumes villous.

HAB. Pine barrens. July-Aug. 4. Fl. purp., with yell. and white.

\section{CLITORIA. Leguminosa.}

1. C. mariana: leaves ternate, ovate, acute, pale beneath; calyx tubular-campanulate, 5-cleft, much longer than the linear bracts; legume torulose.

IIAB. Shady river-banks. Aug. 4. Erect or twin.; fl. large, pale blue.

2. C. virginiana : leaves ternate, orate; calyx 5-parted, scarcely as long as the bracts; segments subulate, diverging; legumes suhensiform.

IIAB. Hedges. Aug. 4. Stem twining; $f$. very large, pale violet.

\section{ROBINIA. Locust. Leguminosa.}

R. Pseuducacia: leaves pinnate, with an odd leaflet; stipules prickly ; racemes pendulous ; teeth of the calyx unarined; legumes smooth.

HAB. About plantations. May. A large tree; $f$. large, wh. in ling racein.

459. TEPHROS!A. Leguminosce.

T. virginica : erect, villous; leaflets numerous, ob long-lanceolate, acuminate; raceme terminal, sub sessile; legumes falcate, villous. 
HAB. Dry woods. July. 4. Stem $12 \mathrm{in.} \mathrm{high:}$ leav. pale $g r . ; f$. wh. and purp.

460. MEDICAGO. None such. Leguminosce.

M. lupulina: spikes ovate; legumes reniform, 1seeded, veined and rugose ; stems procumbent.

HAB. Fields, \&c. May-Oct. ○. Stems diffuse; spikes small, yell.

\section{SYNGENESIA.}

\section{EQUALIS:}

* Florets all ligulate.

461. CICHORIUM. Cal. surrounded with leafy scales. Recept. somewhat chaffy. Pappus plumose, sessile, unequal.

462. APARGIA. Cal. imbricate. Recept. naked, punctate. Pappus plumose, sessile, unequal.

463. LEONTODON. Cal. imbricate, with Haccid scales. Recept. naked. Pappus simple, stipitate.

464. PRENANTHES. Cal. surrounded with leafy scales. Florets few, (5-20.) Recept: naked. Pappus simple, subsessile.

465. LACTUCA. Cal. imbricate, cylindrical; scales membranaceous at the margin. Recept. naked. Pappus simple, stipitate.

466. SONCHUS. Cal. imbricate, swelling at the base. Recept. naked. Pappus simple, sessile. 167. HIERACIUM. Cal. imbricate, ovate. Recept. nearly punctate. Pappus simple, sessile.

468. KRIGIA. Cal. many-leaved, simple. Recєpt. naked. Pappus double; exterior 5-8-leaved; interior of 5,8 , or 24 scabrous bristles.

* Florets all tubular, forming a hemispherical head. 469. ARCTIUM. Cal. globose, with the scales hooked at their extremity. Recept. chaffy. Pappuss setaceous-chaffy.

170. ONOPORDON. Cal. ventricose, imbricate with 
spreading spinous scales. Recept. pitted. Pappus capillary, deciduous, scabrous.

471. CNICUS. Cal. ventricose, imbricate with spinous scales. Recept. hairy. Pappus deciduous, plumose.

172. LlATRIS. Cal. oblong, imbricate. Recept. naked. Pappus plumose, persistent, (mostly coloured.) Seed pubescent, striate, obconic.

473. VERNONIA. Cal. ovate, imbricate. Stig. bifid. Pappus double ; exterior short and chafiy ; interior capillary.

** Florets all tubuiar, erect, forming a nearly level top.

174. KUHNIA. Cal. imbricate, cylindric. Recept. naked. Pappus plumose, sessile. Seed pubescent with nume rous striæ.

475. EUPATURIUM. Cal. imbricate, oblong. Style much exsert, deeply cleft. Recept. naked. Pappus scabrous. Seed 5-striate, or argular.

476. MIKANIA. Cal. 4-6-leaved, 4-6-flowered. Recept. naked. Pappus hairy.

477. CHRYSOCOMA. Cal. imbricate, oblong or hemispherical. Style scarcaly exserter. Kecept. naked. Pappus hairy, scabrous. Seed pubes. cent.

478. CACALIA. Cal. cylindric, scaly at the base. Recept. naked. Pappus hairy.

179. SPAKGANOPHORUS. Cal. subglobose, imbricate; scales recurved at the point. Recept. naked. Seed crowned with a cartilaginous shining cup.

\section{SUPERFLCA.}

* Florets of the ray obsolete.

480. TANACETUM. Cal. imbricate, hemispherical; scales acuminate. Rays of the corolla obsolete, trifil. Recept. naked. Pappus somewhat margined.

161. ARTEMISIA. Cal. ovate, imbricate; scales rounded, connivent. Florets of the ray subulate. Recept. subvillous or naked. Pappus 0 
182. BACCHARIS. Cal. imbricate, cylindric; scales ovate, subcoriaceous. Fertile florets mixed with the perfect. Recept. naked. Pappus hairy.

183. CONYZA. Cal. imbricate; scales often subscarious. Recept. naked. Marginal florets fertile, 3-cleft. Pappus simple and capillary.

484. GNAPHALIUM. Cal. imbricate; scales scarious, mostly coloured. Recept. naked. Florets of the ray subulate; fertile florets entire. Pappus scabrous.

* Florets of the ray ligulate.

185. ERIGERON. Cal. imbricate, subhemispherical. Florets of the ray very numerous and narrow. Recept. naked. Pappus double; exterior minute; interior hairy, of few rays.

186. INULA. Cal. imbricate. Florets of the ray numerous, (yellow.) Recept. naked. Pappus simple, scabrous; (sometimes with a minute exterior chaffy papjus.)

437. ASTER. Cal. imbricate, with the lower scales often spreading. Florets of the ray generally more than 10, rarely fewer, (not yellow.) Recept. naked. Pappus simple.

438. SOLIDAGO. Cal. imbricate, scales connivent. Florets of the ray about 5, (yellow.) Recept. naked. Pappus simple, scabrous.

489. ARNICA. Cal. bemispherical; leaflets equal, longer than the disk. Recept. naked. Pappus simple, hairy. Florets of the ray (yellow) often destitute of anthers.

490. SENECIO. Cal. subcylindric, equal, scaly at the base; scales withered at the points. Reoept. naked. Pappus simple. (Rays sometimes wanting.)

491. TUSSILAGO. Cal. simple, equal, membranaceous, swelling. Recept. naked. Pappus simple, sessile. (Flowers mostly discous.)

192. BOLTONIA. Cal. imbricate. Rays numerous. Recept. conic, punctate. Seeds flat and submarginate. Pappus consisting of many minute 
bristles, with 2 of them opposite, and mostly elongated.

493. CHRYSANTHEMUM. Cal. hemispherical, imbricate; scales with membranaceous margins. Recept. naked. Pappus 0.

494. VERBESINA. Cal. many-leaved; leaflets disposed in a double series. Rays about 5. Recept. chaffy. Pappus 2-awned.

495. ANTHEMIS. Cal. hemispherical; scales nearly equal, with scarious inargins. Recept. convex, with flat rigid chaff. Seed crowned with a membranaceous border.

496. ACHILLEA. Cal. ovate, imbricate, unequal. Rays 5-10, roundish-obcordate. Seeds without pappus ur border.

497. HELIOPSIS. Cal. imbricate; scales suborate, lined. Rays large and linear. Recept. chaffy, conic; chaff lanceolate. Seed quadrangular. Pappus 0.

498. HELENIUM. Cal. simple, many-parted. Rays 3-cleft. Recept. naked, glohose, chaffy on the margin. Seed villous. Pappus of 5-awned scales.

\section{FRUSTRANEA.}

499. HELIANTHUS. Cal. imbricate, subsquarrose, leafy. Recept. chaffy, flat. Pappus chaffy, 2 . leaved, caducous.

500. RUDBECKIA. Cal. subequal, mostly double. Recept. conic, chaffy. Pappus a 4-toothed margin.

501. BIDENS. Cal. subequal, leafy or scaly at the base. Rays often wanting. Recept. chaffy, flat. Pappus consisting of 2-4 retrorsely scabrous awns. Seed quadrangular.

502. COREOPSIS. Cal. double ; each many-leaved; the interior equal, subcoriaceous and coloured. Recept. chaffy ; scales flat. Seed compressed, emarginate, bidentate.

503. AC'TINOMERIS. Cal. simple, many-leaved, foliaceous, subequal. Rays remote, elongated, (4-8.) Recept. small and chaffy ; the leaflets 
embracing the margin of the seed. Seed compressed, margined, crowned with 2 persistent awns.

\section{NECESSARIA.}

504. SILPHIUM. Cal. squarrose; scales broad and leafy. Recept. chaffy. Seed flat, obcordate, emarginate, bidentate.

505. POLYMNIA. Cal. double; exterior 4-5. leaved; interior 10-leaved; leaflets concave. Recept. chaffy. Pappus 0.

506. IVA. Cal. about 5-leaved, or 5-parted. Florets of the ray 5. Recept. hairy. Seed obovate, naked.

\section{SEGREGATA.}

507. ELEPHANTOPUS. Partial Cal. 4-flowered. Florets 5-cleft, ligulate, perfect. Recept. naked. Pappus setaceous.

\section{SYNGENESIA.}

\section{EQUALIS.}

461. CICHORIUM. Succory. Composite.

C. Intybus : flowers axillary, sessile, in pairs; leaves runcinate.

HAB. Fields. July-Sept. 4. Stem 2-3 ft. high, branch., rough ; $f$. large, bl. $\oint$.

462. APARGIA. Composita.

A. autumnalis : scape branching; peduncles squamose ; leaves lanceolate, dentate or pinnatitid, smoothish; caly $x$ elongated.

HAB. Fiells, \&c. June-Nov. 4. Leav. rad.; scape spread., assurg.; $f l$. yell. §.. 
463. LEONTODON. Dandelion. Composita.

1. Taraxacum : exterior scales of the calyx reflexed: leaves runcinate, smooth, dentate. HAB. Pastures, \&c. April-Nov. 4. Smooth; leav. rad.; fl. large, yell.

\section{PRENANTHES. Compositce.}

1. P. alba: radical leaves angular-hastate, somewhat lobed; stem-leaves roundish-ovate, dentate, petiolate; racemes panicled, nodding; calyx 8-cleft, 9-12-flowered.

HAB. Low woods. Aug.-Sept. 4. Stem \&$3 \mathrm{ft}$. high ; fl. white or purp.

a. serpentaria: radical leaves palmate-sinuate; those of the stem un long petioles, with the middle segment 3-parted.

IIAB. Mountains. Aug.-Sept. Fl.purp.

G. nana: stem low; lower leaves 3-parted; middle ones hastate; upper ones ovate or lanceolate: sometimes all are entire.

HAB. High mountains. Aug. Stem 4-15 in. high; fl. purp., often erect.

2. P. altissima: stem branched; leaves 3-lobed, petiolate, angular, denticulate, scabrous on the margin : racemes axillary; flowers nodding; calyx about 5-flowered.

HAB. Woods. Aug. 4. Stem 3-5ft. high; fl. yellowish.

3. P. cordata: stem paniculate above; leaves petio. late, cordate, denticulate, ciliate; the uppermost ones sessile, oblong, entire; flowers racemose, in a loose panicle, nodding; caly $x$ 6-cleft, 6 -flowered.

HAB. Rocky woods. Aug. 4. Stem 3-4 ft. ligh; fl. yellowish.

1. P. virgata: smonth; stem simple ; leaves all lyratesinuate; flowers subsecund, pendulous; calys smooth, 8-cleft, 10-flowered.

HAB. River banks. Sept. 4. Stem $2-4$ fto high, slend. ; $f$. purp.

5. P. racemosa: stem simple; leaves all undivided, smuothish; radical ones oval-lanceolate, denticu- 
late; radical ones closely sessile; raceme oblong; flowers in nodding fascicles; calyx very hairy, 8-9-parted, 9-12-flowered.

HAB. Swamps. Aug.-Sept. 4. Stem 2 ft. high; fl. purp.

\section{LACTUCA. Lettuce. Composita.}

1. L. elongata: leaves smooth; the lower ones runcinate, amplexicaul; upper ones lanceolate, sessile ; flowers paniculate.

HAB. Wet woods. 4. J. July-Aug. Stem 4-6 $f t$. high; pan. large, loose; $f$. yell.

?. L. hirsuta: lower part of the stem and leaves hairy; radical leaves lyrate; segments truncate, subdentate; upper ones partly runcinate-pinnatifid ; flowers in racemes.

HAB. Pennsylvania. Aug. 4. Fl. yell. and purp.

3. L. integrifolia: leaves sagittate, entire, unarmed, amplexicaul ; flowers panicled.

HAB. Road sides. July. \&̊. Stem 3-4 ft. high, smooth; fl. yell.

1. L. sanguinea: leaves amplexicaul, runcinate, glaucous beneath; with the midrib filamentous ; flowers panicled.

HAB. Woods. July-Aug. ơ? Stem $2-3 \mathrm{ft}$. high, purp.; cal. purp. ; fl. criin.

466. SONCHUS. Sow-thistle. Composita.

1. S. oleraceus : perluncles tomentose; calyx smooth; leaves runcinate, dentate.

HAB. Waste places. July-Sept. ๑. Stem 2-3 ft. high, hollow; fl. yell. $\S$.

$\because$ S. arvensis: peduncles and calyx hispid, subumbellate; leaves runcinate, denticulate, cordate at the base; root creeping.

HAB. Banks of rivers. 4. Stem 2 ft. high: glandular-hairy; fl. large, yell. §.

3. S. leucophous: peduncles squamose; flowers racemose ; leaves runcinate, acuminate; stem virgate and panicled.

IlAB. Swamps. Sept. 4. of. Stem 2 ft. high: yl. yellorish. 
4. S. acuminatus: peduncles subsquamose; flowers paniculate; radical leaves subruncinate; cauline ones ovate, acuminate, petiolate, denticulate in the middle.

HAB. Woods. Aug. fo Stem 4-5 ft. high; $f$. small, blue.

3. S. pallidus: raceme compound, terminal; leaves lanceolate-ensiform, amplexicaul, dentate.

HAB. Fields. July. 4. Stem 2-3 ft. high; f. small, yell.

6. S. floridanus : peduncles subsquamose ; flowers paniculate; leaves lyrate-runcinate, minutely dentate, petiolate.

HAB. Road sides. Aug. \&. Stem 3-5 ft. high; pan. long; $f$. sm., blue.

7. S. macrophylhus: perluncles hairy, naked; flowers paniculate; leaves lyrate; cordate at the base, hairy beneath.

HAB. Low grounds. Aug.-Sept. 4. Stem 4-i ft. high; fl. large, blue.

3. S. spinulosus: lenves amplexicaul, oblong, undulate, spiriulnse; flowers somewhat umbelled.

HAB. Salt marsbes. Ang. $\odot$. Stem $2 \mathrm{ft}$. high; pedun. smooth, or subpilose; fl. yell.

467. HiERACIUM. Hawk-weed. Composite.

1. H. venosum: scape naker, paniculate, smooth; leaves obovate-lanceolate a little hairy above, naked beneath, ciliate; the veins coloured; calyx smooth.

HAB. Wonds. July-Aug. 4. Scape 1-2 ft. high; leav. rad.; fl. yell.

9. H. Gronovii : scapie leafy, paniculate ; calyx glandular-hairy; leaves obovate, obtuse, entire, strigose ; midrib beneath very villous.

HAB. Dry hills. Aug.-Sept. 4. Stem $2 \mathrm{ft}$. high, nearly naked; pedicels gland.

3. H. paniculatum: smoothish; stem erect, leafy, whitish tomentose ; pedicels capillary ; leaves lanceolate, naked, dentate, iriembranaceous.

HAl3. Woods. Aug.-Sept. 4. Stem 1 ft. high; leav. smooth; pedicelslong. 
4. H. Kalmii: stem erect, subrillose ; leares sessile. lanceolate, acuminate, acutely and divaricately dentate; panicle subcorymbose; pedicels tomentose.

HAB. Rocky wools. Aug. 4. Siem 2 ft. high; pedicels thick.

э. H. marianum : stem erect, villous; leaves obovate or elliptic, strigose and hispid, villous on the midrib; the lower ones slightly dentate; pedicels and calyx tomentose.

HAB. Sandy hills. Aug.-Sept. Stein 2 ft. high. pan. dense; ped. gland.

\section{KRIGIA. Compositce.}

1. K. virginica : glaucous; primary leaves roundish, entire; the rest lyrate, nearly smooth ; scape 1 flowered, smooth, at length longer than the leaves; calyx smooth.

HAB. Sandy soils. May-July. ○. Scape 1-6 in. high; fl. sm., orange.

2. K. amplexicaulis: glaucous; leaves oblong-orate ; radical ones subdentate, spathulate; scape somewhat leafy, often bifid; branches 2-3-flowered; pappus with many bristles.

HẢB. Woods. June. 4. Stem 1 ft. high; pe. dunc. elong.; $f$ l. large, orange.

469. ARCTIUM. Burdock. Compositc.

A. Lappa: leaves cordate, petiolate, without prickles.

HAB. Road sides, \&c. July-Dec. \&. Leaz. large, spread.; fl. term., purp. $\$$.

470. ONOPORDUM. Cotton Thistle. Composita.

O. Acanthium: scales of the calyx spreading, subulate; leaves ovate-oblong, decurrent, sinuate and spinous, woolly on both sides.

HAB. Waste places. July. \& Plant rehite and woolly; fl. large, solit., purp. \$. 
471. CNICUS. Thistle. Compositce.

* Leaves decurrent.

1. C. lanceolatus : leaves decurrent, pinnatifid, hispid ; the segments divaricate and spinous; calyx ovate, tomentose ; scales lanceolate, spreading.

HAB. Waste places. July-Sept. §. Sten 2-4 ft. high; leav. toment. beneath. $\S$.

\section{* Leaves sessile.}

9. C. altissimus: leaves sessile, oblong-lanceolate, scabrous, tomentose beneath, dentate, ciliate; radical ones pinnatifid; calyx ovate, bracteate; scales ovate-lanceolate, spinous, appressed.

HAB. Old fields. June-Sept. 4. Stem $3-8$ ft. high: Al. subsolit., large, purp. or wh.

3. C. arvensis: leaves sessile; pinnatifid, spinous; stem paniculate; calyx ovate or globose ; scales ovate-lanceolate, mucronate, appressed.

HAB. Road sides, \&c. July. 4. Root creep.; stem 2-3 ft. high; fl. sm., purp. §?

4. C. horridulus: leaves sessile, pinnatifid, acutely laciniate, very spinous, woolly beneath; involucrum many-leaved, 1-llowered, scales acute.

11AB. Fields. June-Aug. s. Stem 1-2 $f t$. high; fl. axil. and term., large, yeilowish.

כ. C. muticus: leaves all pinnatifid, woolly beneath : segments spinulous, somewhat acute; branches naked, 1 -flowered; calyx globose; scales unarmed.

IIAB. Mearlows. Aug.-Sept. 8. Stem subsimp., 3-4 ft. high; cal. reolly.

ij. C. discolor: leaves sessile, lanceolate, pinnatifid, smooth above, white and tomentose beneath; segments 2-lobed, spinous; calyx subglobose; scales appressed, with reflexed points.

HAB. Thickets. July_Sept. 8े. Stem 3-5 ft. high, much branch.; fl. red.-purp.

7. (C. virginianus: stem simple, attenuated, mostly 1 flowered; leaves sessile, lanceolate, hoary and tomentose beneath, very smooth above, remotely dentate; teeth spinous; calyx ovate; scales ap. pressed, with short points, carinite. 
HAB. Woods. June. 4. Stem 3-5 ft. high, slend.; cal. glutin.; fl. purp.

3. C. pumilus : stem hairy; leaves amplexicaul, green on both sides, oblong-lanceolate, pinnatifid; seg. ments irregularly lobed, ciliate and spinous; calyx subglobose, naked; scales appressed, spinous.

HAB. Sandy fields. Aug. of. Stem thick, $1 \mathrm{ft}$. high; $f$. few, large, purp.

9. C. glutinosus: leaves pinnatifid, with divaricate segments; calyx ovate, with unarmed glutinous scales.

HAB. Damp soils. Aug.-Sept. §. Stem 4-6 fi. high;f. small, purp.; cal. webbed.

\section{LIATRIS. Composita.}

1. L. squarrosa : stem simple, pubescent; leaves linear, very long; raceme few-Howered, leafy; calyx large, about 20-flowered; scales leafy, lanceolate, mucronate, rigid and spreading; segments of the florets linear, villous internally.

HAB. Dry woods. Sept. 4. Root tub.; stem 2-3 ft. high; $f$. few', very large.

․ 1. scariosa : stem erect, hairy; leaves lanceolate: pubescent, scibrous along the margin; raceme long; calyx 14-flowered; scales obovate, nearly smooth, with the margin scarious; the lower ones spreading.

IIAB. Sandy hills and woods. Sept. 4. Stem 3-4 ft. high; fl. num., large.

3. L. spicata: leaves linear, entire, smooth, ciliate at the base, nerved and punctate; raceme spiked; scales of the calyx linear, obtuse.

IfAB. Moist mearlows. Aug. 4. Stem 3-5 fie high, smooth; cal. 8- $l$.

A. L. pilosa: stem simple, pubescent ; leares linear, hairy, ciliate; flowers racemose, loose ; scales of the calyx oblong, obtuse; pedicels bracteate.

HAB. Pine barrens. Sept.-Oct. 4. Root bulb.: stem. slend.; fl. sinall.

473. VERNONIA. Compositc.

1. V. noveboracensis: leaves numerous, lanceolate, scabrous, serrulate; corymb fastigiate; scales of the calys fuliform at the extremity. 
HAB. Low grounds. Aug.-Oct. 4. Stem 4-6 ft. high; fl. large, purp.

2. V. procalta: stem angular, densely pubescent; leaves numerous, lanceolate, acutely serrate, pubescent beneath; corymb fastigiate; scales of the calyx ovate, acute, unarmed.

HAB. Meadows. Aug.-Oct. 4. Verytall; $f$. purp.

474. KUHNIA. Compositce.

1. K. eupatorioides: smooth ; leaves petiolate, broadlanceolate, serrate, corymb terminal, few-flowered, crowded.

HAB. Shady woods. Aug.-Sept. 4. Stem 2-3 ft. high : fl. roh. ; cal. 10-ft.

2. K. Critonia: leaves linear, nearly entirc, punctate beneath; panicle long, expanding.

HAB. Rocky banks. Sept. 4. Stern slend., striate, pubes.; fl. whh.; seed striate.

475. EUPATORIUM. Hemp Agrimony. Compositu:

* Calyx 3-5.fowered.

1. E. hyssopifolium: stem erect; lowest leaves opposite, linear, very entire or subdentate, pubsscent, 3 -nerved; corymb subfistigiate; style much longev than the corolla.

HAB. Pine barrens. Aug. 4. Slem 2 ft. high: leav. punc; $f$. wh.

2. E. linearifolium: stem subprocumbent, villous above; stem-leaves opposite, lanceolite-linear, rarely dentate, sometimes fasciculate; style is long as the corolla.

UAB. Sandy fields. Aug.-Nor. 4. Stem 1-? ft. high, subviscid; $A$. wh.

3. E. sessilifoliun : leaves sessile, amplexicaul, distinç, ovate-lanceolate, rounded at the base, very smooth; stem smooth.

HAB. Rocky woods. Aug.-Sept. 4. Stem? ft. high; leav. large; fl. wh.

A. E. Iruncalum : leaves sessile, amplexicaul, distinct, 
lanceolate, truncate at the base, serrate, nearly smooth ; stem pubescent.

HAB. Rocky woods. July-Oct. 4. Leav. punct., coarsely serrate; $f$. wh.

5. E. album: leares subsessile, oblong-lanceolate, scabrous, serrate; interior scales of the calyx long, scarious, and white.

HAB. Pine barrens. Sept.-Oct. 4. Stem 18 in. high, slend., pubes., cal. gland.

6. E. lanceolatum: leaves sessile, distinct, oblonglanceolate, scabrous, deeply serrate at the base; scales of the calyx of one colour.

HAB. Low grounds. Aug.-Nov. Fl. white.

1\%. E. teucrifolium: leaves sessile, distinct, orate, sca. brous, coarsely serrate at the base; uppermost ones entire.

HAB. Low grounds. Aug.-Sept. 4. Stem 2 ft. high; leav. subamplex.; $f$. wh.

3. E. melissoides: leaves petiolate ovate, rather obtuse, serrate, veined, smoothish.

HAB. Pennsylvania. 4. Fl. wh.

3. E. rotundifolium: leaves sessile, roundish-cordate, obtusely serrate, veined; scales of the calyx acuminate.

HAB. Sandy fields. Sept.-Oct. 4. Stem 18 in. high; leav. large; $f$. subfastig.

10. E. pubescens: leaves sessile, distinct, orate, scabrous, veined; the lower ones doubly serrate; upper ones subserrate; stem paniculate, pubescent ; branches fastigiate.

HAB. Sandy woods. Aug.-Oct. 4. Branched; leav. acute; $f$. wh.

11. E. ceanothifolium : leaves petiolate, ovate, acuminate, dentate, triply nerved, smooth.

IIAB. Rocky woods. Aug.-Nov. 4. Stem $2 \mathrm{fl}$. high; leav. large; $f$. wh.

12. E. ovatum: hairy, scabrous; leaves opposite, sessile; ovate, obtusely dentate; corymb fastigiate : calyxes about 8-flowered.

IIAB. Low grounds. July-Aug. 4 . Stem 3-4 ft. high ; fl. wh. ; cal. hairy.

13. E. altissimum: leaves subsessile, lanceolate, 3 - 
nerved, attenuate it each end, pubescent; the in. ferior ones serrate about the middle.

HAB. Sandy woods. Aug.-Oct. 4. Stem 3-r ft. high; fl. white.

14. E. ameenam : leaves on short petioles, opposite and ternate, lanceolate-oblong, acute at each extremity . serrate, nearly smooth, subrugose ; reticulately veined beneath ; corymb crowded; scales of the calyx acute, coloured.

HAB. Mountains. Sept.-Oct. 4. Stem $2 \mathrm{ft}$. high, solid; pedunc. toment.; fl. purp.

15. E. trifoliatum: leaves petiolate, ternate and quaternate, ovate, acuminate, seriate, subscabrous, punctate.

HAB. Woods. Aug.-Sept. 4. Stem 3-4 ft. high, solid; corymb. large, purp.

* Calyx more than 5-flowered.

16. E. purpureun : leaves petiolate by fours or fires, ovate-lanceolate, serrate, rugosely veined, slightly scabrous ; stem hollow.

HAB. Low grounds. Aug.-Sept. 4. Stem 5-6 ft. high, purp.; fl. purp.; cal. 8-leav.

17. E. muculatum s leaves petiolate, by fours or fives, ovate-lanceolate, unequally serrate, pubescent beneath; stem solid, sulcutc.

HAB. Low grounds. Aug.-Sept. 4. Stem 4-5 ft. high, punct.; cal. 5-8-fl.

18. E. verticillatum: leaves petiolate, by threes or fours, ovate-lanceolate, acuminate at each end, unequally serrate, nearly smooth; stem solid

HAB. Wet woods. Aug.-Sept. 4. Stern 3-4 fl. high, slend.; cal. wh.; fl. purp.

19. E. punctatum: leaves petiolate, by fours and fives, acuminate, serrate, scabrous on both sides ; stem solid, terete.

HAB. Mountains. Aug.-Oct. 4. Fl. purp.

20. E. perfoliarum : leaves connate-perfoliate, pubescent.

HAB. Low grounds. Aug.-Sept. 4. Stem $2 f t$. high, branch., vill.; leav. large; $f$. roh.

11. E. celestinum: leaves petiolate, cordate-ovate, ob. 
tusely dentate, triply nerver, subscabrous ; calyx many-leaved, many-flowered; flowers corymbed. HAB. Mountains. Aug.-Sept. 4. Stem 2 fi. high; fl. large, blue, dense.

92. E. aromaticum : leaves petiolate, ovate, acute, obtusely dentate, subscabrous; stem paniculate above: flowers corymbed; calyx simple.

HAB. Dry woods. Aug. 4 . Stem $2 \mathrm{fl}$. high, pubes.; leav. triply nerv.; fl. large, wh.

3. E. ageratoides: leaves on long petioles, subcor. date, ovate, acuminate, dentate, nearly smooth, triply nerved; corymb many-flowered; calyx nearly equal.

HAB. Rocky hills. Aug.-Oct. 4. Stem $2 \mathrm{ft}$. high, smooth; fl. num., wh., small.

476. MIKANIA. Composita.

.1 M. scandens : stem climbing, smooth; leaves cordate. repand-rlentate acuminate, with divaricate unequa! lobes; fluwers corymbed.

HAB. ioist thickets July-Sept. 4. Leav. oppos. ; corymh. axill., piink.

2. M. pubescens : stem climbing, pubescent; leaves cordate, acuminate, angularly dentate, pubescent on both sicles; lobes divaricate, equal.

HAB. Low grounds. Sept. 4. Fl. pale purp.; odor.; cal. pubes.

477. CHRYSOCOMA. Goldy-locks. Compositæ.

C. virgata: herbaceous, smooth; leaves all narrow and linear; stem virgately brinched; branches corymbiferous, fastigiate; caly $x$ oblong, 3-4flowered; scales glutinous and appressed.

HAB. Swamps. 4. Stem 18 in. high, branch.; leav. 1-2 in. long; fl. yell.

478. CACALIA. Composita.

1. C. suaveolens : stem herbaceous; leaves petiolate, hastate-sagittate, serrate, smooth, green on both sides; flowers corymbed, erect; calyx many. flowered. 
HAB. Banks of rivers. Aug. 4. Stem $3-4 \mathrm{ft}$. high; leav. large; fl. wh.; anth. yell.

9. C. atriplicifolia : stem herbaceous; leaves petiolate, smooth, glaucous beneath ; radical ones cordate, dentate; of the stem rhomboidal, subdentate on each side; flowers corymbed, erect; calyx 5flowered.

HAB. Banks of rivers. Aug. 4. Stem 3-6 ft. ligh; pedunc. reh. ; fl. sm., yell.

3. C. reniformis: stem herbaceous; leaves petiolate, smooth, hairy on the veins beneath; radical ones cordate, reniform, repandly dentate; of the stem oblong, dentate, cuneate and very entire at the base ; corymb fastigiate; calyx many-flowered.

HAB. Banks of rivers. July. 4. Stem $5-7 \mathrm{ft}$. high; fl. yell.-zoh.

479. SPARGANOPHORUS. Compositce.

S. verticillatus: leaves linear, verticillate; flowers few, terminal; pappus campanulate, 5 -toothed.

HAB. Swamps. Aug. 4. Stem simp.; $1 \mathrm{ft}$. high, whorl. 6-8 leav.; fl. purp.

\section{SUPERFLUA.}

480. TANACETUM. Tansey. Compositce.

'T. vulgare: leaves doubly pinnate, incisely serrate. HAB. Old fields, \&c. Aug.-Sept. 4. Plant odor.; fl. dense, yell. §.

481. ARTFMISIA. Wormwood. Compositce.

1. A. canadensis: stem herbaceous, paniculate, mostly erect; radical leaves subpinnate. slightly tomentose; cauline ones pinnate, segenents subsetaceous, incised, flat, nearly smooth; llowers subglobose, sessile; scales of the calyx scarious.

HAB. Sandy shores. Aag. H. Sten 3-4 ft. high, brownish; fl. glomerate.

$\therefore$ A. cauduta: sten simple, herbaceous, much branch ed, pyramidal; radical and cauline leaves bipinnate, pubescent; ujper oncs pinnate; scgments 
subsetaceous, alternate, divaricate, somewhat con. vex ; flowers pedicellate, erect, glubose-ovate. HAB. Sea shores. 4. Stem 2 ft. high.

3. A. vulgaris: leaves tomentose beneath; cauline ones pinnatifid; divisions lanceolate, subdentate, acute; floral leaves linear-lanceolate, entire; flowers subsessile, oblong, erect; calyx tomentose.

HAB. Rocky hills. Sept. 4. Stem 2 ft. high; leav. green above. $\S$ ?

482. BACCHARIS. Ploughman's Spikenard. Com. posita.

B. halimifolia: leaves obovate, incisely dentate above ; panicle compound, leafy ; fascicles pedur. culate.

HAB. Sea coast. Sept.-Oct. h. Plant pulve. rulent; leav. thick; pap. long, silkg.

483. CONYZA. Marsh-fleabane. Compositoe.

C. camphorata: leaves ovate-lanceolate, somewhat pubescent, acute, serrate; the serratures mucronate; flowers in crowded corymbs.

HAB. Salt marshes. Aug. 4. Strong-scented; stem 1 ft. high; fl. purp.

434. GNAPHALIUM. Cud-rveed. Composito.

1. G. plantagineum : shoots procumbent; stem simple; rarlical leaves ovate, 3-nerved, mucronate; flowers diøcious.

11AB. Dry hills and woods. Apr.-Sept. 4. Plant dozeny; rad. leav. large; fl. pale purp.

2. G. polycephalum : herbaceous, erect; leaves linear: lanceolate, acute, smooth above, pubescent beneath ; stem paniculate, tomentose ; corymbs terminal, crowded.

HAB. Woods and low grounds. Aug. ○. Strongscented, branch.; $f$. clust., pale yell.

3. G. margaritaceum: herbaceous; leaves linear. lanceolate, acuminate, alternate; stem branched at the top; corymb fastigiate ; flowers pedicellate. HAB. Fields and woods. Aug. 4. Stem 1-2 ft. high, woolly; fl. large, few, reh. 
7. G. uliginosum: stem much branched; branches spreading, woolly; leaves linear-lauceolate; flowers in terminal, leafy crowded hearls.

HAB. Low grounds, \&c. Aug. ๑. Stem low, spread.; cal. yellowish.

5. G. americanum : stem herbaceous, erect, branched; leaves obovate-spathulate, pubescent beneath; flowers axillary and terminal, in glomerate spikes. HAB. Fields and woods. June. ๑. Stem 6-8 in. high; fl. yell.-roh.

G. G. purpureum: herbaceous; leaves linear-spathulate, tomentose beneath; stem erect, simple; flowers sessile, glomerate, axillary and terminal.

HAB. Fields. June. 4. Stolonif.; stem 8-12 in. high; $f$. purplish.

7. G. germanicum: stem erect, proliferously branched upward; leaves lanceolate, cottony ; flowers small, in dense round heads, axillary and terminal.

11AB. Fieldls. July. ๑. Stem 6-8 in. high; branch. fero, horizontal; fl. yell.

3. G. decurrens: stem erect, much branched; leaves linear-lanceolate, very acute, decurrent, white and woolly beneath, naked above; flowers in dense terminal roundish clusters.

IIA B. Hills and fields. Aug. 4. Stem $2 \mathrm{ft}$. high; leav. green above; M. yell.

9. G. sylvaticum: sten erect, simple, downy ; flowers in a leafy spike; leaves linear-lanceolate, downy。 particularly beneath.

HAB. Woods. July. 4. Stem $12 \mathrm{in.}$ high; base subdecumb.; cal. brownish.

185. ERIGERON. Flea-bane. Compositu.

1. E. bellidifolium : hairy, gray; radical leaves obovate, subserrate; stem leaves remote, oblonir-ovate, amplexicaul, entire; stem 3-5-flowered; lay's neariy iwice as long as the hemispherical calys.

HAB. Hills. May. 4. Stem erect, simp., 12-18 in. high; fl. pule purp.

2. E. integrifolium : stem simple, leafy, smooth; leavez lanceolate, entire, 3-nerved ; flowers corymbe.l; calyx hemispherical; scales acute. 
HAB. Woods, \&c. June-Aug. 4. Stem $2 f t$. high, ; leav. rarely subdent.; fl. woh.

3. E. philadelphicum: pubescent ; leaves cuneate-oblong, subserrate; those of the stem semiamplexicaul; florets of the ray capillary, as long as the disk; stem branched above, many-flowered.

HIAB. Old fields. June-Oct. 4. Stem 2-3 $f t$. high, slend.; $A$. pale purp.

4. E. purpureum : pubescent; leares oblong, dentate, amplexicaul ; upper ones very entire; peduncles corymbed, thick; the inferior ones elongated; rays twice as long as the calyx.

HAB. Woods. June. 4. Stem $2 f t$. high; $f$. large, purp.

5. E. strigosum : hairy and strigose; leaves lancenlate, attenuate at ench end, with a few coarse teeth in the middle, or entire; flowers in a corymbose panicle.

HAB. Fields and woods. June-Oct. ô. Stem 2-3 ft. high, sulc.; rays capill., wh.

6. E. heterophyllum: radical leaves roundish-ovate, with large teeth, petiolate; stem leaves lanceolate, acute, serrate in the midrlle; corymb terminal.

HAB. Meadows. June-Oct. ż. Stem 2-3 fto high; leav. large; $f$. wh.

* Subgenus CENOTUS. Calyx oblong ; rays very numerous, minute; pappus simple.

7. E. canadense: stem hairy or hispid, paniculate : leaves lanceolate; lower ones subserrate; rays crowded, very short.

HAB. Fields and cultivated grounds. Aug.-Nor. ๑. Stem 4 in. -5 ft. high. Variable.

\section{INULA. Composita.}

1. I. Helenium: leaves amplexicaul, ovate, rugose, tomentose beneath; scales of the calyx.

HAB. Road sides. July-Aug. 4. Stem 3-4 ft. high; leav. large; $f$. yell. 
* Subgenus CHRYSOPSIS. Calyx imbricated; rays of the corolla mostly yellow; anthers naked at the base; pappus double; exterior paleaceous minute; interior scabrous, many-rayed; seeds obovate, villous.

2. I. mariana: hairy; leaves oblong-lanceolate, serrate; upper ones sessile, acute ; lower spathulate, roostly obtuse ; corymb simple; calyx viscidly pubescent.

HAB. Woods. Aug. 4. Stem $18 \mathrm{in.high;} \mathrm{rays}$ 10-12 oblong.

3. I. falcata: woolly and villous; leaves sessile, linear, acute, rigid, subfalcate; calyx subtomentose.

HAB. Pine-barrens. Sept-Uct. 4 . Stem 6-12 in. high; fl. small.

4. I. graminifolia: silky; leaves lanceolate, linear, acute, entire, nerved; corymb compound; stem leafy towards the summit.

HAB. Sandy woods. Aug.-Oct. 4. Stem $2 \mathrm{ft}$. high; leav.long, subrigid.

487. ASTER. Starwort. Composito.

* Calyx rohite, green at the summit; florets of the ray about 5 , white.

1. A. solidaginoides: leaves linear-lanceolate, entire, scabrous on the margin; flowers sessile aggregate; calyx imbricate, with the scales obtuse, appressed.

HAB. Dry woods. July-Aug. 4. Stem $2 \mathrm{ft}$. high ; fl. fastig.-corymb.

2. A. conyzoides : leaves oval-lanceolate, acute, serrate towards the summit, triply nerved; the lower ones attenuate at the base; upper entire ; scales of the calyx oval, obtuse, appressed, slightly reflexed at the surnmit.

HAB. Woods and copses. July-Aug. 4. Stem $2 \mathrm{ft.}$ ligh; leav. thick; cal. cylind.

* * Florets of the ray numerous; pappus single.

\section{$\dagger$ Leaves entire.}

3. A. hyssopifolius : leaves linear-lanceolate, 3-nerved, punctate, acute, scabrous on the margin; branches 
fastigiate, clustered ; florets of the ray about 5 calyx imbricate, half as long as the disk.

$H A B$. Sandy fields. Aug. H. Stem $18 \mathrm{in} . h i g h$ slend. ; fl. small, wh., or purplish.

4. A. subulatus: smooth; stem much branched from the base, paniculate; leaves long and linear, very acute; uppermost subulate; calyx subcylindric, with subulate scales; radical forets minute.

HAB. Salt marshez. Aug.-Nov. ๑! Stem 12 -18 in. high; fl. small, purp.

5. A. foliolosus: leaves linear-lanceolate, attenuate at each end, acuminate; stem pubescent, paniculate, erect; branches few-flowered; calyx imbricate, with linear acute appressed scales.

HAB. Dry fields. Aug.-Oct. 4 . Fl. small, white.

6. A. paludosus: stem simple ; leaves linear subulate, smooth, amplexicaul; peduncles few, one-flowered, pubescent, leafy; calyx squarrose.

HAB. Open swamps. Aug. 4 . Stem 3-5 fl.; f. large, blue; cal. subfoliaceous.

\%. A. tenuifolius: leaves linear-lanceolate, tapering at each end, very entire; margin slightly scabrous ; stem smooth, branched, erect, with 1-flowerect branches; scales of the calyx acute, loose.

HAB. Fields. Sept. 4. Stem 2 ft. high, pubes. above; $f$. sm., pale purp.

ß. dumosus: branches paniculate, with very short leaves.

HAB. Fields. Sept. Fl. wh. or pale purp.

$\%$ ericoides: leaves linear; those on the branches short and approximate; stem smooth.

HAB. Rocky fields. Sept. Lozer leaves long; very smooth; $f$. pale purp.

3. A. inultiflorus : leaves linear, entire, nearly smooth: somewhat ciliate; stern diffusely branched, pubescent, calyx imbricate squarrose, with oblong, ciliate scales.

HAB. Rocky fields. Sept. 4. Stem 2 ft. high, very pubes.; flo reh.; disk yell.

ß. ciliatus: leaves distinctly ciliate; those of the branches very short.

HAB. With the preceding. 
9. A. sparsiflorus: very smooth; stem sparingly branched, somewhat flexuous; leaves long and succulent; the inferior ones sublanceolate-linear; superior subulate; branches one-flowered, leafy ; scales of the calyx lanceolate, acuminate, appressed; rays numerous, shorter than the calyx. HAB. Salt marshes. Aug.-Oct. 4. Sten 12 in. high; fl. large, purp.

10. A. concolor: leaves oblong-lanceolate, entire, whitish-pubescent on both sides; stem simple, erect, pubescent; raceme terminal; scales of the caly $x$ lanceolate, silky, appressed.

HAB. Pine barrens. Oct. 4. Foot often tuber.; stem virg.; fl. large, blue.

11. A. salicifolius: leaves linear-lanceolate, nearly entire; stem smonth, paniculate above; calyx loose, imbricate; scales acute, spreading at their points.

HAB. Low grounds. Sept. 4. Stem tall; $f$. middle-sized, reddish-tlue.

19. $\Lambda$. cestivus : leaves lanceolate, subamplexicaul, attenuated at the apex; margin scabrous; stem branched from the bise, erect, bispid; branches hairy; scales of the calyx loose, linear, acute, equal.

HAB. Dry swamps. Sept. 4. Stem 2 ft. high: fl. middle-sized; rays blue.

13. A. novae anglict: leaves linear-lanceolate, amplexicaul, auriculate at the base ; stem hairy and paniculate; flowers subsolitary on the branchlets, somewhat fustigiate; scales of the calyx loose, linear-lanceolate, rather longer than the disk.

IIAB. Fields. Aug.-Oct. 4. Stenb 4-6 ft. high ; fl. large, purp.-bl.; rays numerous.

14. A. cyaneus: leaves linear-lanceolate, amplexicaul, smooth; stem branched, with the branches expanding, very smooth; flowers in paniculate ritcemes; scales of the calyx loose, lanceolate, its long as the disk.

HAB. Along fences. Sept. 4, Stem 3 fl. high, subgluuc.; fl. blue, large.

15. A. phlogifulius: leaves lanceolate, cordate, amplexicaul, pubescent beneath, scabrous on the B b 2 
margin; stem simple, pubescent; panicle terminal, loose, few-flowered; scales of the caly $\mathbf{x}$ loose, lanceolate.

HAB. Sandy woods. Sept. 4. Stem 12-13 in. high, slend.; $f$. violet.

16. A. patens : leaves oblong-lanceolate, ciliate, cordate and amplexicaul, scabrous and hairy on both sides, those on the branches minute; branches spreading, elongated, few-flowered; scales of the calyx spreading, lanceolate.

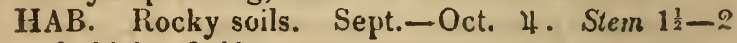
ft. high; $f$. blue.

It Leares lanceolate and ovate; the lower ones serrate.

a Flowers corymbed.

17. A. nudiflorus: stem simple and smooth; leaves sessile, ovate-lanceolate, subacuminate, all sharply serrate, scabrous above; corymb few-flowered; peduncles pubescent, naked, mostly 1-Howered; calyx hemisphericat, closely imbricate; scales linear-oblong, ciliate.

HAB. Swamps. 4. Stem 2-3 ft. ligh; pedunc. long; fl. large, pale purp.

18. A. Radula: leaves lanceolate, serrate, acuminate, rugous and very scabrous; stem erect, angular, simple; corymb terminal ; calyx imbricate ; scales lanceolate, rather obtuse, subsquarrose.

11AB. High mountains. Sept. 4. Fl. middle sized; rays short, white.

19. A. strictus : leaves sessile, narrow-lanceolate, serrate, scabrous; stem one or few-flowered at the sumunit ; scales of the calyx imbricate, appressed, oblong, acute, about equal to the disk.

HAB. High mountains. Sept.-Oct. 4. Stem 4-8 in. high; $\AA$. pale violet.

20. A. surculosus: stem simple, low, slender, minutely pubescent ; lower leaves linear-lanceolate, entire, or subserrate, scabrous above ; upper ones linear, amplexicaul; corymb $3-5$ llowered, somewhat naked; calyx imbricate, subsquarrose; scales ciliate, linear-oblong; inner ones obtuse; rays uumerous. 
HAB. Margin of swamps. 4. Root stolonif., creep.; fl. large, purp.

21. A. spectabilis: leaves oblong-lanceolate, somewhat scabrous, subamplexicaul; lower ones serrate in the middle; branches corymbed; calyx hemispherical, with a subglandular pubescence, foliaceous, and squarrose; leuflets ciliate, cuneateovate, partly acute.

HAB. Sandy swamps. Oct. 4. Branch. 2-3-fl.; fl. large, blue.

ß. bcllidifolius: leaves oblong-obovate, serrate, corymb nearly simple, or with the branches mostly 1 -flowered.

HAB. With the preceding. Siem not hairy and glandular above.

22. A. serotinus : leaves oblong-lanceolate, acuminate, sessile, smooth, scabrous on the margin; inferior serrate; branches corymbose, smooth ; branchlets one-flowered; scales of the calyx lanceolate, acu. minate, spreading.

IIAB. Low grounds. Sept.-Oct. 4. Stem $3 f t$. high ; fl. large, blue.

23. A. puniceus: leaves amplexicaul, lanceolate, serrate, somewhat scabrous; branches paniculate; cally $x$ loose, longer than the disk; leaflets linearlanceolate, subeciual ; stem hispid.

HAB. Brackish swamps. Sept. 4. Stem $6 \mathrm{ft}$. high, purp. ; fl. large, purp.

2.1. A. fluribundus : leaves subamplexicaul, linceolate; inferior serrate; stem smooth; branches corymbed; branchlets many-flowered, hairy; sciles of the calyx loose, lanceolate, imbricate.

HAB. Pennsylvania. 24.

35. A. novi belgii: leaves subamplexicaul, lanceo. late, smooth, scabrous on the margin : inferior subserrate; branches subdivided; calyx loose, imbricate; leaflets linear-lanceolate ; stem terete, smooth.

HAB. Fields. Aug.-Sept. 4. Stern 3 ft. high; fl. middle sized, purp.

26. $A_{4}$ acuminatus: leaves broad-lanceolate, narrowed and entire at the base, unequally serrate above, long-acuminate ; stem simple, flexuous, angular ; 
panicle divaricately dichotomous; scales of the calyx loose, linear.

HAB. Mountains. Sept. 4. Stem 12 in. high; leav. large; $f$. $x$ h.

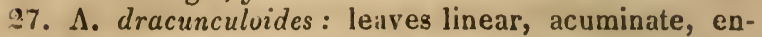
tire; lower linear-lanceolate, slightly serrate; branches corymbose; calyx imbricate; stem nearly smooth.

HAB. Fields. Sept.-Oct. 4. Stem 3 ft. high; fl. small, reh.

b Flowers panicled.

98. A. amplexicaulis: leaves ovate-oblong, acute, amplexicaul, cordate, serrate, smooth ; stem pauiculate, smooth; branches 1-2-flowered; scales of the calyx closely imbricate.

HAB. Dry woods. Sept.-Oct. 4. Stem 18 in. high ; $f$. blue.

29. A. laevigatus: leaves subamplexicaul, broad-lanceolate, subserrate, smooth; stem much branched, smooth; branches many-flowered; scales of the calyx lanceolate, loose, equal to the disk.

IIAB. Low grounds. Oct. 4. Stem 2-3 $\mathrm{fl}$. high; $f$. pale purp.

30. A. versicolor: leaves subamplexicaul, broad-lanceolate, subserrate, smooth; radical ones serrate in the middle; stem much branched, smooth; scales of the calyx lanceolate, loose, shorter than the disk.

IIAB. Fields. Sept.-Oct. 4. Stem 2 ft. high; fl. pale blue or zchite.

31. A. mutabilis: leaves subamplexicaul ; upper ones lanceolate, acuminate, very entire; inferior lanceolate, narrow at the base, serrate; branches virgate; calyx loose, shorter than the disk; stem smooth.

IIAB. Fields and diry woods. Sept. 4. Stem 2-3 fi. high; fl. purp.

32. A. laevis : leaves subamplexicaul, remote, oblong, very entire, shining; radicul ones subserrate; branches simple 1-flowered, scales of the calyx subcuneiform, acute, thickened at the tip ; stem smooth angular. 
HAB. Low grounds. Oct. 4. Stem 2 ft. high: f. large, blue.

33. $\Lambda$. concinnus: leaves lanceolate, subamplexicanl: inferior ones subserrate, smooth; stem simple, paniculate above; calyx closely imbricate.

HAB. Fields. Sept. 4. Stein $2 \mathrm{ft}$. high; $\pi$. purple.

34. A. bellidiflorus: leaves amplexicaul, narrow-lanceolate, scabrous above ; inferior ones subserrate: stem much branched; scales of the calyx lanceolate, spreading.

HAB. Pennsylvania. A var. of A. spectabalis?

35. A. tardiflorus: leaves sessile, spathulate-lanceolate, serrate, smooth, attenuate at the base; margin reflexed; branches divaricate; calyx loose; scales lanceolate-linear, subequal, snooth.

HAB. Low grounds. Sept.-Oct. 4. Fl. middlesized, violet.

36. A. Tradescanti: leaves lanceolate, serrate, sessile, smooth; branches virgate ; calyx loose, imbricate; stem terete, smooth.

HAB. Fields. Aug.-Oct. Stem $3 \mathrm{ft}$. high; $f$. small, wh. or blue.

$\beta$. recurvatus : leaves narrow-lanceolate ; stem recurved.

37. A. eminens : leaves linear-lanceolate, acuminate, scabrous on the maryin; inferior ones subserrate; stem paniculate; branches one-flowered; calys loosely-imbricate ; scales lanceolate.

HAB. Fields. Sept. 4. Fl. lilac.

38. A. laxus: leaves linear-lanceolate, acuminate, scabrous on the margin ; the lower ones subserrate, those of the stem subreflexed, of the branches much spreading; stem luose, paniculate above; calyx imbricate; scales lanceolate, acute, reflexed at the apex.

HAB. Fields. Sept._Oct. 4. Fl. small, zehite.

39. A. simplex : leaves lanceolate, acuminate, scibrous on the margin, those of the stem serrate at the end, of the branches very entire; stem quite smooth, paniculate above ; calyx loose ; scales linear-subulite.

IIAB. Pennaylvania. 4. Fl. wh. 
40. A. polyphyllus : leaves linear, very entire; radical ones oblong, subserrate; stem much branched, pubescent ; calyx loose.

HABB. Pennsylvania. 24. Fl. white.

41. A. junceus: leaves lanceolate-linear, sessile, smooth ; lower ones subserrate ; stem paniculate, smooth ; branches virgate; calyx imbricate.

HAB. Low grounds. Oct. 4. Stem tall, slend.; f. ilesh-col.

6. lanceolatus : stem branched, diffuse, rather smooth.

42. A. fragilis : leaves linear, acuminate, very entire; radical ones serrate, oblong ; branches corymbosepaniculate; scales of the calyx appressed; stem nearly smooth.

HAB. Old fields. Sept. 4. Stem 2 ft. high; $f$. small, wh.

13. A. miser: leaves sessile, lanceolate, serrate, smooth; scales of the calyx icute; disk equal to the ray; stem somewhat villous.

HAB. Old fields. Sept.-Oct. Fl. small, white. a. divergens: leaves elliptical-lanceolate, those of the stem elongated ; branches spreading.

ß. diffusus : leaves all proportioned.

$\%$ pendulus : leaves of the branches rather remote; branches very divaricate, pendulous.

c Leaves cordate, serrate.

44. A. undulatus : leaves oblong-cordate, amplexicaul, very entire, hairy, somewhat undulate; inferior ones ovate, cordate, subserrate; petioles winged; stem paniculate, hispid; branches secund, leafy, 1-flowered.

HAB. Dry woods, \&c. Sept. 4. Stem $2 \mathrm{ft}$. high ; fl. rniddle-sized, bl.

45. A. sagittifolius: leaves oblong-lanceolate, acuminate, sessile, serrate in the middle ; radical ones oblong, corlate-sagittate, serrate, petiolate ; stem branched, smooth; scales of the calyx loose, lanceolate.

HAB. In rocky woods? Sept. 4. Fl. middlesized, blue.

46. A. paniculatus : leaves ovate-lanceolate, subserrate. netiolate, smooth; radical ones ovate-cordate: 
serrate, scabrous, petiolate; petioles naked ; stem. mnch branched, smooth ; branches hairy ; calyx loose, subimbricate.

HAB. Fields and woods. Sept. 4. Stem 2-3 $f t$. high; $f$. pale blue. Near A. undulatus.

47. A. cordifolius : leaves cordate, hairy beneath, acutely serrate, petiolate; petioles winged; stem paniculate, smoothish; panicle divaricate; calyx loose, subimbricate.

HAB. Rocky woods. Sept. 4. Stem 2 ft. high; fl. rather small, pale blue.

18. A. corymbosus : leaves ovate, acutely serrate, acuminate, smoothish ; inferior ones cordate, petiolate; petioles naker!; stem smooth, corymbosefastigiate above; branches hairy; calyx oblong, imbricate; scales obtuse, closely appressed.

HAB. Dry woods. July-Aug. 4. Stem $2 \mathrm{ft}$. high; $f$. middle-sized, wh.

49. A. macrophyllus: leaves ovate, petiolate, serrate, scibrous; upper ones ovate-cordate, sessile ; inferior cordate, petiolate; petioles somewhat margined; stem branched, diffuse; calyx cylindrical; closely imbricate ; scales oblong, acute.

IIAB. Woods. Ang. H. Stem $2 \mathrm{ft}$. high; $\mathrm{fl}$ pretty large, wh. or pale $b l$.

* * Pappas double; flowers mostly corymbed.

50. A. linariifolius: leaves linear, mucronate, thick. without nerves or punctures, carinate, scabrous, rigid; those of the branches recurved; stem subrecumbent; branches fistigiate, elongated, 1 flowered ; calyx imbricate, as long as the disk.

HAB. Dry fields, and on rucks. Sept.-Oct. A foot high; fl. violet.

i) 1. A. rigilus : leaves linear, mucronate, subcarinate, rigid; margin scabrous-ciliate; stem-leaves reflexed, of the branches spreading, subulate; stem erect, somewhat branched above; branches 1 . flowered, corymbed; calyx imbricite, half the length of the disk; scales carinate, rather obtuse; rays about 10 -flowered, reflexed.

HAB. Sandy tields. Sept. 4. A foot high; $A$. middle-sized, violet. 
52. A. linifoirus: leaves linear, without nerves or punctures, scabrous, reflexed-spreading; branches corymbose-fastigiate, leafy; caly $x$ imbricate, short; rays equal to the disk.

HAB. Dry woods, \&c. Sept.-Nor. \&. Stem 1-2 ft. high; fl. large, wh. or pale blue.

53. A. humilis: leaves oblong-ovate, acuminate, aeute at the base, scabrous on the margin; petiole short; stem smooth, dichotomously paniculate above, few-flowered; calyx loose, subimbricate.

HAB. Woods, \&c. Aug. 4. Stem 1-2 ft. high; leav. large; fl. wh.

54. A. amygdalinus: leaves lanceolate, attenuate at the base, acuminate, scabrous on the margin; stem simple, corymbose-fastigiate at the summit; calyx loose, imbricate : scales lanceolate, obtuse.

HAB. Fields and low grounds. July-Aug. 4.A foot high; $f$. reh.

488. SOLIDAGO. Golden-rod. Composita.

* Pacemes secund; leaves triply nerved.

1. S. canadensis : stem villous, leaves lanceolate, serrate, triply nerved, scabrous; racemes paniculate, secund, recurved; rays short.

HAB. Old fields, \&c. Aug.-Sept. 4. Stem 3-4 ft. high; leav. large.

2. S. procera : stem villous, erect; leaves lanceolate, serrate, scabrous, villous beneath; racemes spiciform, erect, when young drooping; rays short. HAB. Low grounds. Sept.-Oct. 4. Stem 4-6 ft. high.

3. S. serotina : stem erect, terete, smooth ; leaves linear-lanceolate; racemes paniculate, secund; peduncles pubescent.

HAB. Meadows. Sept. 4. Stem 4 ft. high: young leaves ciliate.

4. S. gigantea: stem erect, smooth ; leaves lanceolate, serrate, scabrous on the margin, obscurely triplynerved; racemes paniculate, secund; peduncles hairy ; rays short.

HAB. Low meadows. Sept.-Oct. 24. Stem 4-7 ft. high, purplish. 
J. S. ciliuris: stem erect, smooth; leaves lanceolate, subtriply nerved, smooth, except on the margin, subserrate; racemes paniculate, secund; pedun. cles smooth ; bracts ciliate; rays short.

HAB. Borders of woods. Aug. 4. Stem $3 \mathrm{ft}$. high, angul.; radical. leav. long.

3. S. reflexa: stern erect, villous; leaves lanceolate, subserrate, triply nerved, scabrous, reflexed; branches paniculate, subsecund, reflexed.

HAB. Fields. Aug. 4.

7. S. lateriflora: stem erect, somerwhat hairy ; leaves lanceolate, subtriply nerved, smooth, scabrous on the margin; lower ones subserrate; racemes paniculite, subrecurved, secund.

HAB. Meadows. Aug. 4. Stem $2-3$ ft. high; rays elongated.

* Rucemes secund; leaves veined.

צ. S. aspera : stem erect, terete, hairy ; leaves ovate and somewhat elliptical, very rough, rugous, serrate, without nerves; racemes paniculate, secund. HAB. Fields. Sept. 4. Stem 3 ft. high; leav. acute; racerr. dense.

3. S. altissina: stem erect, hirsute; leaves lanceolate, the lower ones deeply serrate, very rough, rugons; panicles secund; rays short.

HAB. Old fields. Sept.-Oct. 4. Stem 3-5 fl. high. - Variable.

10. S. rugosa: stem erect, hairy ; leaves lanceolate, very rough and rugous; the lower ones with ap. pressed serratures; racemes paniculate, widely spreading.

IIAB. Stony fields. Sept.-Oct. H. Stem $4 \mathrm{ft}$. high.

11. S. scabra: stem erect, hairy, sulcnte ; deaves oblong, attenuate at each end, acuminate, smooth above, rugous and scahrous beneath, with ap. pressed serratures in the millile; racemes s:cund.

HAB. Meadows. Aug.-Sept. 4. Stem $3 \mathrm{fth}$ high.

12. S. nemoralis: stem erect, woolly; cauline leave$\mathrm{C}$ c 
lanceolate, hispid, rery entire ; radical ones sui cuneiform, serrate; racemes paniculate; secund.

HAB. Barren fields. Sept. 4. Plant gray, 1$2 \mathrm{ft}$. high.

13. S. patula: stem erect, smooth; leaves elliptical. serrate, smooth; lower ones oblong-spathulate; racemes paniculate, secund, spreading; peduncles pubescent.

HÄB. Dry woods. Sept. 4. Stem \& ft. high, virgate; racemes short.

14. S. ulmifolia: stem erect, smooth, striate; leaves elliptical, deeply serrate, acuminate, villous be. neath ; radical ones obovate; racemes paniculate, secund; peduncles villous; rays short.

HAB. Low grounds. Aug.-Sept. 4. Stem $3 \mathrm{ft}$. high; leaves large.

15. S. arguta: stem erect, smooth; leaves smooth, acutely and unequally serrate; cauline ones elliptical ; radical ovate-oblong; racemes paniculate, secund; rays elongate.

HAB. Meadows. Aug. 4. Stem $2 f t$. high.

16. S. juncea: stem erect, smooth; leares lanceolate, smooth except on the margin ; lower ones serrate; racemes paniculate, secund.

HAB. Meadows and dry woods. Sept. 4. Stem $3 \mathrm{ft}$. ligh, slender.

17. S. elliptica: stem erect, smooth ; leaves elliptical, smooth, serrate; racemes paniculate, secund; rays of middling length.

HAB. Dry woods. Sept. 4. Stem $3 \mathrm{ft}$. high; lear. large and broad.

18. S. sempervirens: stem erect, smooth; leaves linear-lanceolate, somewhat fleshy, smouth, very entire, scabrous on the margin; racemes paniculate, secund; peduncles hairy.

HAB. Swamps. Sept.-Oct. 4. Stem 2 ft. high. subglauc.; rays narrow, elong., 5-6.

19. S. virgata: stem smooth, quite simple; leaves subcuneate-lanceolate, obtuse, very smooth, upper ones very entire, appressed to the stear. growing smaller above; branches of the panicle elongate, racemed at the summit; peduncles smooth, secund. 
HAB. Swamps. Oct. 4. Stem atten., $3-4 \mathrm{ft}$. high; lower leav. subserrate; fl. small.

30. S. odora: stem erect, pubescent, slender; leaves linear-lanceolate, very entire, smooth, scabrous on the margin; racemes paniculate, secund.

HAB. Rocky hills and dry woods. July-Aug. 4. Stem subvirg.; plant odorous.

$$
\text { * * Racemes crect. }
$$

31. S. speciosa: stem smooth, simple, or with virgate branches; leaves lanceolate, entire, somewhat fleshy; lower ones very broad; radical subserrate; racemes erect and compound, pubescent ; rays about 5 , elongate.

HAB. Sharly woods. 4. Stem 4-6 ft. high, sulc.; leav. large; pedunc. short.

22. S. bicolor: stem hairy; leaves elliptic, hairy; lower ones serrate; branches leafy; racemes short, dense, erect; scales of the calyx obtuse.

HAB. Dry hills and woods. July-Aug. 4. Stem 1-2 ft. high; rays white.

23. S. stricta: stem erect, smooth; cauline leaves lancenlate, very entire, smooth, scabrous on the margin; radical ones serrate; racemes paniculate, erect; peduncles smooth.

HAB. Sandy woods. Sept. 4. Stcm 2 ft. high; panic. dense.

24. S. petiolaris : stem erect, villous; leaves elliptic, somewhat scabrous, petioliate; racemes erect; rays elongate.

HAB. Sandly fields. Sept.-Oct. 4 . Stem branch.; racem. numerous, short.

25. S. lanceolata : stem angular, hairy, much branched; leaves lanceolate-linear, entire, $3-5$-nerved, scabrous on the margin, minutely hispid on nerves beneath ; corymbs terminal, fastigiate ; rays $15-$ 20 , as long as the disk.

HAB. Meadows. Sept. 4. Stem s-4 ft. high; f. glomerate; cal. ovate.

26. S. tenuifoliu: stem angular, much branched, smonthish; leaves linear, very narrow, numercus, scabrous-ciliate on the margin, obscurely 3 . 
nerved, punctate above; corymbs fastigiate ; rays ahout 10 , exserted.

HAB. Sandy fields. Sept.-Oct. 4. A foot high; fl. glomerate; axils leafy.

27. S. caesia: stem smooth, glaucous, erect; leaves lanceolate, smooth; racemes erect; rays of mid. dling length.

HAB. In dry woods. Sept. 4. Stem slend., : ft. high, subvirgate; pedicles scab.

28. S. fexicaulis: stem flexuous, sinooth, angular ; leaves ovate-lanceolate, acuminate, serrate, glabrous; racemes axillary; rays half as long agair: as the calyx.

HAB. Fields and woods. Sept. 4. Sicm slend. purp., 2 ft. high; racem. short.

29. S. latifolia: stem somewhat flexuous, angular, smooth; leaves broadly ovate, acuminate, deeply. serrate, smooth, petioles winged; racemes axillary.

HAB. Dry Woods. Sept.-Oct. 4. Stem 18 in. high; leav. 2 in. broad, atten. at base.

30. S. hispida : stem erect, hispid, scabrous; leaves lanceolate, scabrous, very entire; radical ones serrate ; racemes erect ; rays of middling length.

HAB. Fields and woods. Sept. 4. Resembles No. 27.

31. S. laevigata: stem erect, smooth ; leaves oblong lanceolate, fleshy, very entire, very smooth ; racemes paniculate, erect : peduncles scaly, villous; rays elongate.

HAB. Salt marshes. Sept.-Oct. 4. Stem 4$5 \mathrm{ft}$. high; rays about 10 ; radical leaves subob. ovate.

32. S. limonifolia: stem smooth; leaves lanceolate, somewhat fleshy, very entire, smooth; racemes paniculate, suberect, nearly naked; peduncles scaly, pubescent; rays about 10.

HAB. Marshes. Aug. 4. Stem 3-4 ft. high : pedunc. mostly 1-flow.

33. S. viminea : stem erect, subpubescent; leaves linear-lanceolate, membranaceous, attenuate at the base, smooth, scabrous on the margin; lower ones subserrate; racemes erect; rays elongate. 
HAB. Low grounds. Sept. 4.

34. S. rigida: stem hairy and scabrous; leaves ovateoblong, rough, with minute hairs; those of the stem very entire; lower ones serrate; flowering branches paniculate; racemes compact; rays elongate; scales of the calyx obtuse.

HAB. Rocky woods. Aug.-Sept. 4. Stem tall; leaves rigid; $f$. very large.

35. S. noveboracensis: radical leaves ovate oblong, petiolate; stem somewhat naked, fastigiately branched.

HAB. Meadows. Sept. 4. Stem $3 \mathrm{ft}$. high, thick; leav. rough; fl. large.

36. S. squarrosa: stem thick, pubescent above ; leaves smooth; lower ones very broad, spathulate-oval, serrate, acute; upper ones lanceolate-elliptic ; racemes glomerate, rigid, and pubescent; calyx squarrose, many-flowered; rays 10-12, elongate.

HAB. Kocky hills. Sept. 4. Stem 2 ft. high; A. very large.

489. ARNICA. Compositce.

1. nudicaulis: hairy; radical leaves decussate and op posite, oblong-oral, subdentate; stem nearly leafless, divided at the summit into several 1-flowered branches.

HAB. Woods. June. 4. Stem 1-2 ft. high; leav. large, routgh beneath; $f$. large, yellow.

490. SExECIO. Gruundsel. Compositce.

* Florets of the rays wanting.

12. S. hieracifolius: stem virgate, paniculate; leares oblong, amplexicaul, unequally and deeply toothed ; dentures acute; calyx smooth ; seeds hairy. HAB. Fields, \&c. Aug.-Oct. ๑. Stem 2-3 ft. high, subscab.; caly.x ventric., large; $f l$. wh.

2. S. vulgaris: leaves mostly amplexicaul, sinuatepinnatifid, toothed; stem paniculate, erect, angular. HAB. Waste grounds. Ang. ๑. Stem $18 \mathrm{in}$. nigh; leav. thin; fl. pale yell. §.

3. S. elongatus: radical leaves spathulate, aftenuate. C c 2 
into a petiole, serrate; stem leaves pinnatifid toothed, very remote; peduncles elongate, umbellate, corymbed.

HAB. Rocky banks. July-Aug. 4. Resembles S. Balsamitce.

* Flowers radiate.

4. S. gracilis : radical leaves on very long petioles, orbicular, subcordate, crenate; stem-leaves few, very remote, linear-oblong, dilated at the base, incisely dentate; peduncles very short, hairy, subumbellate; calyx smooth; rays few, very short.

HAB. Rocky banks. May-Aug. 4. A foot high; fl. small.

5. S. aureus: radical leaves ovate, cordate, serrate, petiolate; stem leaves pinnatifid, dentate, terminal segment lanceolate; pelluncles subumbellate, thick.

HAB. Rocky woods. May. 4. Stem 18 in. high; umb. simp.; fl. yell.

6. S. Balsamitae : radical leaves oblong, serrate, petiolate; lower cauline leaves lyrate-pinnatifid, serrate; upper ones pinnate, dentate; flowers somewhat umbellate; stem and peduncles villous at the base.

HAB. Meadows, \&c. June. 4. Stem 1-2 ft: high; leav. subpubes.; fl. yelloz:.

7. S. heterophyllus: woolly and downy ; leaves on long petioles, ovate-spathulate and ovate, rather acute and pinnatifid; cauline ones $2-3$, linear, pinnatifid; flowers corymbed.

HAB. Rocks. May-June. 4. A foot high; $f$. bright yellow.

3. S. obovatus: radical leaves obovate, crenate-serrate, petiolate; cauline leaves pinnatifid, dentate; lowers subumbellate, on long peduncles; stem rather smooth.

HAB. Rocky woods. May-June. 4. A foot high: leav. thick; fl. yell.

491. TUSSILAGO. Colt's-foot. Compositic.

1. T. frigida: scape fastigiate, many-flowered, brac. 
teate; leaves roundish-cordate, unequally tooth. ed, woolly beneath.

HAB. High mountains. June. 4. Af span high; rays zeh.; disk purp.

9. T. Farfara: scape 1-flowered, scaly ; leaves cordate, angular, toothed, woolly beneath.

HAB. Banks of rivers. April. 4. A span high: f. yell., appearing before the leav. §?

\section{BOLTONIA. Compositce.}

1. B. glastifolia: lower leaves serrate; flowera on short peduncles; seeds obcordate, conspir.uously winged, pubescent; awns of the pappus of nearly equal length.

HAB. Banks of rivers. July-Aug. 4. Stem 2 -3ft. high; leav. lin., allern.; fl. flesh-col.

3. B. asteroides: leaves very entire; Howers on long peduncles ; seeds oval, smooth, scarcely awned.

HAB. Wet places. July. 4. Stem 1-2 ft. kigh : leav. alter., lanceol.; fl. few, pink.

493. ChrysanthemuM. Ox-eye Daisy. Compositce.

C. Leucanthemum: leaves amplexicaul, lanceolate, serrate, laciniate-dentate at the base; stem erect; branching.

HAB. Fields, \&c. May-Nov. 4. Stem 12... $18 \mathrm{in.} \mathrm{high;} \mathrm{fl.} \mathrm{large} \mathrm{wh.}$.

494. VERBESINA. Compositu.

I. Siegesbeckia: leaves opposite, ovate, lanceolate acuminate at each end, acutely serrate; panicle brachiate, irregularly many-flowered at the summit ; rays $1-3$.

HAB. Dry woods. Sept. 4 . Stem $9-4$ ft. hicklt 4-wing; leav. triply nerv.; fl. yell.

495. ANTHEMIS. Chamomile. Compositue.

f. A. arvensis: receptacle conic; chaff lanceolate; seed crowned with a margin; leaves lanceolate; segments lanceolate-linear? 
HAB. Fields and waste grounds. July-Nov. $\odot$. Stem much branch.; fl. wh., disk yell. $\S$.

2. A. Cotula: receptacle conic; chaff bristly; seed naked; leaves bipinnate; segments subulate, 3 parted.

IIAB. Waste grounds. June-Nor. ๑. Erect; fl. in termin. corymb.; $f$. wh. $\S$.

\section{ACHILLEA. Milfoil. Composita.}

1. A. Ptarmica : Ieares linear, acuminate, acutely and equally serrate, smooth.

HAB. Dry swamps. Aug. 4. Fl. white. §?

2. A. Nillefolium: leaves bipinnatifid, hairy; seg. ments linear, toothed, mucronate; calyz sulcate. HAB. Fields, \&c. June-Sept. 4. A foot high: corymb dense; $f$. wh. or rose-col. $\S$.

\section{HELIOPSIS. Composita.}

H. lovis: leaves opposite, ovate, serrate, triply-nerved. HAB. Banks of rivers. July-Aug. 4. Stem $2 \mathrm{ft}$. high; leav. smooth; fl. large, yell.

493. HELENIUM. False sunflower. Composita.

1. autumnale: leaves lanceolate, serrate, decurrent; flowers corymbed; florets of the disk 5-cleft, of the ray flat, reflexed.

IIAB. Low meadows. Aug.-Sept. 4. Stem $\sim$ $-3 f t$. ligh, winged; $f l$ yell.

\section{FRUSTRANEA.}

499. HELIANTHUS. Sunflower. Composita.

$$
\text { * Florets of the disk dark purple. }
$$

1. H. atrorubens: hispid; stem naked towards the summit; loosely paniculate; leaves spathulate. oblong-ovate, crenate, triply-nerved, scabrous on the upper surface; scales of the calyx ovate-lanceolate, as long as the disk.

H.AB. Dry soils. Aug.-Sept. 4. Stem $3-4 \mathrm{fto}$ high; leav. opp., very long; rays yell. 
3. H. angustifolius: stem slender, slightly scabrous ; leaves narrow-lanceolate, revolute on the margin, scabrous, entire, glaucous beneath; upper ones alternate; scales of the calyx linear-lanceolate, ciliate, expanding; chaff 3-toothed.

HAB. Pine barrens. Oct. 4. Stem 3-5 ft. high; branch. few; leav. 4-8 in. long; fl. small.

** Florets of the disk yellowish.

$\dagger$ Leaves opposite.

3. H. mollis: stem smooth below, scabrous above, leaves orate-lanceolate, acute, serrate, scabrous on the upper surface, pubescent and hoary be neath ; flowers few, terminal.

HAB. Swamps. Aug. 4. Stcm 3-6 ft. high: leav. very acute; $f$. few ; cal. hairy.

4. H. divaricatus: stem smooth, branched; leaves subopposite, sessile, ovate-lanceolate, triply-nerved, scabrous above, smooth beneath; panicle trichotomous, slender; flowers very small.

HAB. Rocky woods. Aug. 4. Stem 4-6 ft. high; leav. subacuminate.

5. H. frondosus: stem smooth below; leaves ovate, acutely serrate; peduncles scabrous; calyx squarrose, undulate, leafy, ciliate; rays 8 .

HAB. Woods. Aug.-Sept. 4. Stem 4-5 fi. high; fl. small.

6. H. trachelifolius: leaves ovate-lanceolate, acumir nate, serrite, triply-nerved, very scabrous above and beneath; scales of the calyx linear-lanceolate, ciliate, the exterior ones longer.

HAB. Rocky woods. Aug.-Sept. 4. Stem 3. -4 ft. high; fl. middle-sized.

\section{$\dagger \dagger$ Upper leares alternate.}

7. H. altissinus: leaves alternate, ovate-lanceolate, serrate, scabrous, triply nerved, attenuite at the apex, petiolate; petioles ciliate; scales of the calyx lanceolate, ciliate.

HAB. Low grouncis. Sept. 4. Stem 4-6 fi. high, purp.; rays 16 ; chaff green.

8. H. giganteus : leaves alternate, lanceolate, serrate 
scabrous, obscurely triply-nerved, subsessile, attenuate at each end, ciliate at the base; scaies of the calyx lanceolate, ciliate.

HAB. Low grounds. Aug.-Sept. 4. Stem 56 ft. high, subscab. ; pan. loose, ferv-fl.

9. H. decapetalus: leaves ivate, acuminate, remotely serrate, triply-nerved, scabrous; scales of the calyx lanceolate, subequal, subciliate ; rays $10-12$.

IIAB. Rocky woods. Aug. 4. Stem $4-5 \mathrm{fl}$. high; florver's large.

10. H. multiflorus: leaves triply-nerved, scabrous : lower ones cordate; upper ovate; rays manyflowered; scales of the calyx lanceolate.

IIAB. Rocky woods. Aug.-Sept. 4. Stem and pedunc. scab.; rays very num.

500. RUDBECKIA. Composita.

* Calyx imbricate; chaff mucronate.

1. R. purpuren: scabrous ; lower leaves broad-ovate. narrowed at the base, remotely toothed; cauline ones lanceolate-ovate, nearly entire, acuminate at each end; florets of the ray very long, deflexed. bifid.

HAB Mountains. June-July. 4. Stem $4 \mathrm{ft}$. high; fl. large; rays purp. ; disk brown.

* Calyx subequal; chaff unarmed.

2. R. pinnata: leaves all pinnate; lower segments sometimes 2-parted; pappus entire; stern sulcate, hispid.

HA B. Pennsylvania. July-Oct. 4. Fl. large, ycll.; roys long, reflex.; disk wirp.

3. R. digitata: lower leaves pinnate; segments pinnatifid; upper ones simple, pinnate, the highest 3 -cleft; pappus crenate; stem smooth.

HAB. Mountains. Aug. 4. Siem 5-6 ft. high: lcav. thin; fl. yell.; pap. subtruncate.

4. R. laciniata: lower leaves pinnate; segments 3 lobed; upper ones ovate; pappus crenate ; stem smooth.

HAB. Swamps. Aug. II. Stem 5-8 ft. high: loreer leav. 3-foliate; fl. yell. 
3. R. triloba: hispidly pilose; stem paniculate; branches divaricate, many-flowered, leafy ; leaves lanceolate, acuminate at each end, serrate; the lower ones 3-lobed; leaflets of the calyx linear, deflexed, as long as the ray.

HAB. Mountains. Aug.-Sept. 4. Stem 4-5 fi. high; rays yell.; disk dark purp.

5. R. fulgida: stem hispid ; branches long, virgate, 1-flowered; leaves oblong-lanceolate, denticulate, hispid, narrowed and slightly cordate at the base; calyx leafy, as long as the ray; disk hemispheri. cal ; chaff lanceolate.

HAB. Mountain meadows. Aug. 4. Stem 2$3 \mathrm{ft}$. high; rays $12-14$, yell.

7. I. hirta: very hairy; stem virgate, somewhat branched, 1-flowered; leaves spathulate, lanceolate, triply nerved, serrate, hairy; scales of the calyx imbricate, in a triple series, shorter than the ray; chaff obovate, acute.

HAB. Mountains. Aug. 4. Stem 2-3ft. high, subsimp.; $f$. solit., yell.; rays 14.

501. BIDENS. Burr-marygold. Composita.

1. B. cernua : flowers subradiate, cernuous; exterior calyx as long as the flower; leaves lanceolate, sub. connate, dentate.

IIAB. Ditches, \&c. Aug.-Oct. ๑. Stem 1-\% ft. high; rays yell., often ranting.

5.. minima: leaves lanceolate, serrate, sessile; flow, ers erect.

11.13. Low wet places. Aug. Stcm 4-8 in. high; fl. very small.

?. B. chrysanthemoides: Howers radiate, cernuous; rays thrice as long as the nearly equal calyx; leaves oblong, attenuate at each end, dentate, connate at the hase.

II.1B. Wet places. Aur.-Oct. ๑. Stem 1-i ft. high; fl. large; rays 3 , lanceol.

3. 13. frondosa: flowers discoid; exterior calyx six times as long is the flower; its leaflets ciliate at the bise; lower leaves pinnate; upper ones ternate, lanceolate, serrate. 
HAB. Shady wet places. Aug. ○. Stem $2 \mathrm{fl}$. high, branch.; rays 0 ; seeds 2 -awned.

4. B. connata: flowers discoid; exterior calyx thrice as long as the flower; cauline leaves ternate; lateral leaflets connate; floral ones oblong-lanceolate.

HAB. Meadows, \&c. Aug.-Sept. ○. Stem 2 -3 ft. high, smooth; leav. subglauc.

כ. B. bipinnata: flowers subradiate ; exterior calyx as long as the interior; leaves bipinnate; segments linear-pinnatifid.

HAB. Fields. July-Sept. ○. Stem 8-12 in. high; leav. finely divid.; rays 0 , or $1-3$.

6. B. Beckii : stem subsimple; submersed leaves, divided into many linear and dichotomous segments; flower erect, solitary, terminal.

HAB. Deep water. Aug. 4 ? Stem $2-5 \mathrm{ft}$. long; emersed leav. lanceol. or trifid; fl. radiate.

502. COREOPSIS. Tickseed-sunflower. Compositce.

* Leaves opposite, undivided.

1. C. rosea: small, very smooth; stem simple; leaves linear, entire; axils leafy ; flowers few, longperlunculate, axillary and terminal; seeds very entire, naked.

HAB. Swamps. Aug. 4. A foot high; leav. subconnate; rays 3 -10oth., red.

* Leaves opposite, dividerl.

9. C. Irichosperma: smooth; leaves subquinate, pin. nate; segments linear-lanceolate, serrate; flowers corymbed; leaflets of the exterior calyx ciliately serrate; rays entire; seerls cuneate, $2-4$-toothed. HAB. Cedar swamps, \&c. Aug.-Sept. है. Stem $2 \mathrm{ft}$. high, dichot.; rays lanceol., yell.

3. C. tripteris: smooth; leaves petiolate, lanceolate, very entire; radical ones pinnate; of the stem ter. nate; rays entire; seeds obovate, naked at the apex.

HAB. Mountain meadows. Aug. 4. Stem $4 \mathrm{ft}$ high, terete: Al. small, yell. 
*** Leaves alternate.

4. C. gladiata: stem smooth, dichotomous above; leaves narrow-lanceolate, very entire, thick, attenuated into a petiole; seed obovate, winged; the wings serrulate ; pappus 2-bristled.

HAB. Sphagnous swamps. Sept. 8. Stem 2 ft. high; rays \&, 3-tooth., yell.

5. C. aspera : leaves lanceolate-linear, rough ; upper ones alternate, inferior opposite; stem 1-flowered. $\mathrm{HAB}$. Maryland.

503. ACTINOMERIS. Composita.

A ? squarrosa: stem erect, winged, pubescent and paniculate above; leaves lanceolate, serrate, scabrous; calyx spreading, loose ; receptacle subglobose, at length squarrose.

IIAB. Swamps. Aug. 4. Stem 3-4 ft. high; leay. altern.; rays iong, wh. or yell.

\section{NECESSARIA.}

504. SILPHIUM. Composits.

1. S. ternatum : stem terete, smooth; leaves verticillate by threes, petiolate, lanceolate, unequally subdentate, somewhat scabrous, ciliate at the base ; upper ones scattered and sessile; panicle dichotomous ; calyx ciliate.

HAB. Mountain meadows. July. 4. Stem 16 ft. high; fl. corymb., loose, yell.

2. S. trifoliatum : stem 6 -angled, smooth ; leaves verticillate by threes, ovate-lanceolate, unequally toothed and serrate, scabrous; upper onps sessile; panicle trichotomous.

HAB. River sivamps. Aug. 4. Stem 5-6 fto high; lower leav. subovate; rays 14, yell.

3. S. perfoliatum : stem quadrangular, smooth; leaves opposite, connate, ovate, serrate.

HAB. Mountains. Aug. 4. Slem $6 \mathrm{ft}$. high, petiol. decurrent; rays 24, yell.

D d 
505. POLYMNIA. Composita.

1. P. canadensis: viscid and villous; leaves denticulate, acuminate ; lower ones pinnatifid ; upper 3 . lobed or entire.

HAB. Shady rocks. July. 4. Stem 2-3 ft. high; leav. thin; $f$. in term. clusters, yell.

2. P. Uvedalia: leaves opposite, 3-lobed, acute, attenuate into a petiole, decurrent, the lobes angular-sinuate ; rays elongate.

HAB. Fields, \&c. July. 4. Stem 3-5 ft. high; leav. scab. beneath; fl. larger.

506. IVA. Bastard Jesuit's bark. Composita.

1. frutescens : fruticose ; leaves opposite, ovate-lanceolate, deeply serrate, subscabrous; flowers depressed-globose.

HÄB. Salt-marshes. Aug.-Sept. h. Stem much branch., 3-4 ft. high; leav. thick.

\section{SEGREGATA.}

507. ELEPHANTOPUS. Elephant's-foot. Composita.

1. E. carolinianus: leaves of the root and stem ob. long, tapering at the base, hairy; stem brancher, leafy, hairy.

HAB. Dry soils. Sept. 4. Stem 2 ft. high; $f$. in term. clust., purp.

\section{GYNANDRIA.}

\section{MONANDRIA.}

* Anther adnate, subterminal and persistent. Polliviu affixed by the base, composed of angular grains, elastically cohering.

508. ORCIIIS. Cor ringent; upper lip vaulted. Lip dilated, with a spur beneath. Pollinia 2, terminal, adnate.

509. HABENARIA. Cor. ringent. Lip spurred at the base beneath. Glands of the stalks of the 
pollinia naked and distinct; cells of the stalks adnate, or separated.

* Anther persistent, parallel with the stigma. Pollinia affixed to the summit of the stigma, composed of angular grains, or farinaceous.

510. GUODYERA. Cor. ringent; the 2 lower petals placed beneath the gibbous lip, which is undivided at the extremity. Column free. Pollen angular.

511. NEOTTIA. Cor. ringent; the 2 lower petals placed beneath the lip, which is beardless; interior petals connivent. Column apterous. $\mathrm{Pol}$ len farinaceous.

512. LISTERA. Cor. irregular. Lip 2-lobed, pendent. Column apterous. Anther fixed by its base.

** Anther terminal, inserted, persistent. Pollinia farinaceous or angular, affixed by the base or below the extremity.

513. POGONIA. Petals 5, distinct, without glands. Lip sessile, cucullate, internally crested. Pollen farinaceous.

514. TRIPHORA. Petals 5, distant, equal, and connivent, without glands. Lip unguiculate, cucullate. Column spathulate, flattened, apterous. Pollen farinaceous.

515. CALOPOGON. Pctals 5, distinct. Lip behind (or inverted,) unguiculate; the lamina bearded. Column free. Pollen angular.

516. ARETHUSA. Petals 5, connate at the base. Lip beneath growing to the column, cucullate above, and crested internally.

**** Anther terminal, moveable, deciduous. Pollinia at length becoming waxy.

517. TIPULARIA. Petals spathulate, spreading. Lip sessile, entire, with a conspicuous spur at the base beneath. Column apterous, porrected, free. Anther operculate, persistent ; pollinia 4 , parallel. 
518. MALAXIS. Petals 5, narrower than the lis spreading or deflexed. Lip flattened, cindi. ded, sessile, often exterior. Pollinia 4, $\mathrm{p}^{2}$ lel with each other, fixed to the stigma by their extremities.

319. CORALLORHIZA. Pctals equal and connivent. Lip mostly produced at the base. Column free. Pollinia 4, oblique, (not parallel.)

520. APLECTRUM. Petals equal, connivent. Lip unguiculate, not produced at the base. Anther situated below the summit of the column; pollinia 4, oblique, lenticular.

\section{DIANDRIA.}

7.1. CYPRIPEDIUM. Lip ventricose, inflated, saccate. Petals 4 ; the under one bifid. Column terminating in a petaloid lobe.

\section{HEXANDRIA.}

522. ARISTOLOCHIA. Cal. 0. Cor.1-petalled, ligulate, with a ventricose base. Caps. 6-celled, many-seeded, inferior.

\section{DODECANDRIA.}

523. ASARUM. Cal. subcampanulate, 3-4-cleft. Cor. 0. Anthers adnate to the middle of the filaments. Caps. inferior, 6-celled, crowned with the calyx.

\section{GYNANDRIA.}

\section{MONANDRIA.}

508. ORCHIS. Orchidec.

1. O. spectabilis: lip obovate, undivided, crenate, retuse; petals straight, the lateral ones longer; horn clavate, shorter than the germen; bracts longe: than the Hower; stem leafless. 
HAB. Shady rocks. May-June. 4. Leav. rad., large; fl. large, purp. and wh.

2. 0 ? flava : lip ovate, entire, partly crenulate; horn attenuate, filiform and about the length of the germen; spike crowded; bracts longer than the flower.

HAB. Meadows. June. 4. $\mathrm{Fl}$. pale orangeyell., rather small.

3. O? obsoleta : lip oblong, lanceolate, undivided; petals erect; horn obtuse, scrotiform, as long as the germen.

IIAB. Shady places. July. 4, Bracts very short.

4. O ? tridentata: lip lanceolate, 3-toothed at the extremity ; petals obtuse ; horn filiform, clavate, ascending, longer than the germen.

HAB. Swamps. July. If. Stern 1-2 ft. high,

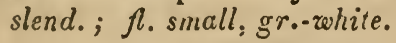

\section{HABENARIA. Orchidea.}

1. H. ciliaris: lip oblong-lanceolate, pinnately ciliate, twice as long as the petals; horn longer than the germen.

HÂB. Swamps. Aug. 4. Stem 2 ft. high; spike dense; $f$. bright orange.

2. H. blephuriglottis: lip lanceolate, ciliate, as long as the upper petal; horn much longer than the germen.

HAB. Swamps. July. 4. Stem 2 ft. high; $A$. pure white.

3. Il. cristuta: lip oblong, pinrately ciliate; petals rounded; the two lateral ones toothed; horn shorter than the germen.

IIAB. Swanps. July. 4. Leav. lanceol.; $A$. crowded, rather small, yell.

4. II. psycodes: lip 3-parted; segments finely divided; petals obtuse ; horn filiform-clavate, ascending, longer than the germen.

HAB. Swamps. July-Aug. 4. Spike long; $\pi$. middle sized, grcenish-woh.

5. II. integra: lip oblong, entire, longer than the inner petals; spur longer than the germen, acute

D d 2 
at the point; stem leafy; bracts shorter than the flowers.

HAB. Swamps. July. 4. Flozers orange yell., sinaller than in No. 1.

o. H. huronensis : lip lanceolate, acuminate, entire, and incurved; petals subulate and connivent; spur about the length of the lip, incurved; petals flat; stem leafy.

HAB. Wet mearlows. Aug. 4. Stem 1-2 ft. high; spike dense, long; f. greenish, small.

7. H. dilatata : lip linear, very entire, rather obtuse, rounded and dilated at the base; horn as long as the lip, shorter than the germen; bracts as long as the flower; stem leafy.

HAB. Mountain woods. July. 4. Stem 2-4 ft. high, very thick; $f$. gr. or wh.

3. H. fuscescens : lip ovate, toothed at the base ; petals spreading; horn subulate, as long as the germen : bracts longer than the flower.

HAB. Mountain meadows. July. 4. Siem thick; spike long; $f$. purp.-yell.

9. H. herbiola : lip oblong, obtuse, toothed at the base; palate 1-toothed; horn filiform, shorter than the germen; bracts longer than the flower.

HẢB. Swamps. June. 4. Stem $1 \mathrm{ft}$. high; $f$. small, yelloreish.

10. H. bracteata: lip linear, emarginate, and obscurely 3 -toothed at the extremity; petals subconnivent; lateral ones ovate and broader; horn obtuse, scrotiform; bracts spreading, twice as long as the flower ; leaves subovate or oblong.

HAB. Shady woorls, \&e. June. 4. Stem 8-12 in. high, leafy; spike loose; $f$. green.

11. H. orbiculata: lip linear, very entire, obtuse; the 3 upper petals connivent; the 2 lateral ones spreading, oblique at the base; horn longer than the germen; scape with 2 orbicular leaves at the base.

HAB. Wet meadows. June. 4. Scape 12-18 in. high; leav. very large; $f$. loose, gr.-rohite.

12. H. macrophylla: lip lanceolate, entire, acuminate; spur longer than the germen, terete, nearly 
straight ; upper petals ovate, acute ; scape with 2 broad-oval suberect leaves at the base.

HAB. Meadows. June. 4. Stem $1 \mathrm{ft}$. high; leav. large; $f$. gr. -yell.

13. H. fimbriata: lip 3-parted, scarcely longer than the petals ; segments cuneiform, ciliately fimbriate ; lateral petals ovate, fimbriate-toothed; horn filiform, clavate, longer than the germen.

HAB. Meadows. July. 4. Stcm 2 ft. high; $f$. large, beantiful purp.

14. H. grandiflora: lip dependent, twice as long as the petals, 3-parted ; segments cuneiform, fimbriate, the middle one largest, with the fimbriæ connivent; lateral petals fimbriate ; spur ascending, clavate, longer than the germen; leaves oval-oblong.

HAB. Wet mearlows. June. 4. Stem $2 \mathrm{ft}$. high, thick; fl. very large, purp.

15. H. incisa : lip 3-parted; segments cuneiform, incisely toothed, the intermediate one emarginate; lateral petals obtuse, subdentate; horn subulate. ascending, longer than the germen.

HAB. Meadows. July. 2. Stem 2-4 ft. high; f. purple.

16. H. fissa: lip 3-parted; segments cuneiform, dentate; intermediate one 2-lobed: horn filiform, clavate at the extremity, ascending, longer than the germen.

HAB. Mountain meadows. July. 4. Stem tall; fl. dark purple.

510. GOODYERA. Orchidece.

1. G. pubescens: radical leaves ovate, petiolate, reticulate; scape sheathed, and with the flowers pu. bescent ; lip ovate, acuminate; petals ovate.

HAB. Woods. July-Aug. 4. Leav. radical, dark green with wh. veins; $f$. wh.

2. G. repens: radical leaves ovate, petiolate, reticulate; scape sheathed, and with the flowers pubescent; flowers unilateral ; lip and petals lanceolate.

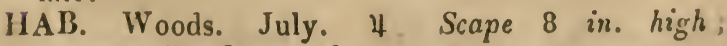
leav. small; $A$. gr,-wh. 
1 . NEOTTIA. Ladies' tresses. Orchidece.

1. N. tortilis : radical leaves linear ; scape sheathed: flowers spirally secund; lip somewhat 3-lobed, the middle lobe larger and crenulate.

HAB. Meadows and woods. July. 4. Scape slend., 12 in. high; $f$. num., wh.; root fascic.

3. gracilis: radical leaves ovate, caducous, membranaceous.

HAB. Dry woods. Scape very slend., smooth.

2. N. cernua: leaves linear-lanceolate, nerved; stem sheathed; spike densely flowered; flowers recurverl-drooping; lip oblong, entire, acute.

HAB. Boggy grounds. Aug.-Oct. 4. Stem 812 in. high, pubes. above; $f$. gr.-wh.

3. major: stem tall, somewhat leafy; flowers very large.

HAB. Woods. Sept. Stem 2 ft. high; fl. 3 times as large as in the common variety.

$\gamma$ ? latifolia: stem short; leaves oblong-lanceolate, subradical, flat.

IAB. Mountain meadows. Stem $4-8$ in. high,; leav. broad; lip ovate, obtuse.

512. LISTERA. Tway-blade. Orchidea.

1. I. cordata: stem with only 2 opposite roundishcordate ; raceme loose ; column without any appendage behind; lip elongate, 2 -toothed at base, deeply bifid; the segments subdivaricate and acute.

HAB. Sphagnous swamps. May. 4. Stem 4$8 \mathrm{in}$. high; raceme $7-15-f_{0}$; $f$. minute, gr.-purp.

2. I. ? convallarioides: stem with only 2 opposite ovalroundish leaves, pubescent above; raceme fewflowered (4-6); column porrected ; lip oblong, dilated, and obtusely 2 -lobed at the extremity.

HAB. Swamps. 4. Stem 6 in. high, slend.; $A$. much larger than in No. 1.

513. POGONIA. Orchidece.

1. I. ophioglossoides: root fibrous; scape furnished 
with an oval-lanceolate leaf and a foliaceous bract near the flower; lip fimbriate.

IIAB. Swamps. July. 4. Root fascic.; scape 1 ft. high; fl. solit., large, purp.

2. I'. verlicilluta: leaves 5, oblong-lanceolate, verticillate; flower solitary; the 3 exterior petals rery long, linear; interior shorter, oblong, obtuse; lip 3-lobed, middle segment undulate.

IIAB. Swamps. July. 4. Stem 8-12 in. high: leav. term.; ext. pet. greenish-brozen.

514. TRIPHORA. Orchidec.

T. pendula : root tuberous; stem leafy, about 3-flowered at the summit ; leaves ovate, alternate ; flowers pedunculate; peduncles at length recurved.

HAB. Rocky woods. Sept. 4. Stem a span high, succul.; $f$. purp.

515. CAlOPOGON. Orchidece.

C. pulchellus: radical leaves ensiform; scape fewflowered.

HAB. Srramps. July. 4. Stem $1 \mathrm{ft}$. high; fl. 3-4, large, purp. ; root bulb.

516. ARETHUSA. Orchidea.

A. bulbosa: leafless; root bulbous; scape sheathed, 1 -flowered.

HAB. Swamps. June. 4. Stern 8-12 in.high, fl. solit., large, purp.

517. TIPULARIA. Orchidec.

'T. discolor.

HAB. Pine barrens. July. Root bulb.; leaf solit., ovate, petiol., plicate; fl. racenn., fere, nodding, greenish.

518. MALAXIS. Orchidea.

1. M. liliifolia: leaves 2 , ovate-lanceolate ; scape triangular; interior petals filiform, reflexed; lip. concave, obovate, acute at the tip. 
HAB. Woods and swamps. June. 4. Leav. large, rad.; scape 6-10 in. high; fl. gr.-rohite.

\section{* Subgenus. MICROSTYLIS.}

Lip sessile and concave, erect, the summit truncate and bidentate; column minute; anthers 2; pollinia 3.

3. M. ophioglossoides: leaf solitary, ovate, amplexicaul; stem pentagonal.

HAB. Swamps. June. 4. Stem a span high, with 1 leaf; $f$. minute, gr.-white.

519. CORALlORHIZA. Dragon's claw. Orchidea.

1. C. verna : petals linear-lanceolate, spreading; lip oblong, without spots, bidentate at the base; apex recurved, ovate; spur obsolete, adnate.

IIAB. Sphagnous swamps. May. 24. Leafless; root coralloid; scape 5-6 in. high; fl. small, yellore-gre.

2. C. odontorrhiza : lip oval, undivided, obtuse, spotted; spur obsolete, adnate; capsule subglobose.

HAB. Shady woods. Aug.-Sept. 4. A foot high, leafless, sheathed; fl. purplish.

3. C. multiflora: scape many-flowered $(15-30)$; lip cuneate-oval, 3-parted, recurved, spotted; spur conspicuous, adnate.

HAB. Shady woods. Aug.-Sept. Scape 12-15 in. high; $f$. purp. and spotted.

\section{APLECTRUM. Orchidea.}

1. hyemale.

HAB. Woods. May. 4. Root bulb.; leaf solit., ovate, striate; scape $12 \mathrm{in.} \mathrm{high,} \mathrm{vugin.;} A . \mathrm{gr}$. purp.; lip 3-cleft, not spotted.

\section{DIANDRIA.}

521. CYPRIPEDIUM. Ladies' slipper. Orchidea.

1. C. candidum: stem leafy; leaves oblong-lanceo. late; lobe of the style lanceolate, rather obtuse; lip shorter than the lanceolate petals, compressed. HAB. Pennsylvania. May. 4. Fl. rh. Resembles C. Calceolus. 
2. C. parviflorum: stem leafy; lobe of the style triangular, acute ; exterior petals ovate-oblong, acuminate, interior linear and contorted; lip shorter than the petals, compressed.

HAB. Woods. May-June. 4. Pet. gr.-brown; lip yell., spotted.

3. C. pubescens: stem leafy ; lobe of the style triangular-oblong, obtuse; exterior petals ovate-oblong, acuminate ; interior very long, linear, contorted ; lip shorter than the petals, compressed.

HAB. Meadows and woods. May-June. 4. Stem 1-3 fl.; $f$. gr.-yell, spotted; leav. pubes.

4. C. spectabile: stem leafy; lobe of the style elliptical-cordate, obtuse ; exterior petals broad-oval, obtuse ; lip longer than the petals, cleft before.

HAB. Swamps. June. 4. Stem 2-3ft. high; $2-3$ fl., thick; lip wh. and purp.

5. C. arietinum: stem leafy ; lobe of the style orbicular ; petals 5 , the 2 lower ones linear-lanceolate, deflexed; the 2 lateral linenr, spreading; upper one oblong-ovate, acute; lip as long as the petals, inversely conical.

HAB. Wet woorls. May. 4. Stem 6-8 in. high; A. small; pet. gr.-brown; lip. retic.

c. C. acaule: scape leafless, 1-flowered; radical leaves 2, oblong, obtuse ; lobe of the style round. ish-rhomboidal, acuminate, deflexed; petals lanceolate; lip shorter than the petals, cleft before. IIAB. Woods. May-June. 4. Scape $1 \mathrm{ft}$. high; f. very large ; lip purp.

\section{HEXANDRIA.}

522. ARISTOLOCHIA. Birthwort.

1. A. Sipho: leaves cordate, acute; stem twining; peduncles 1-flowered, furnished with an ovate bract; corolla ascending, the border 3-cleft and equal.

IIAB. Mountains. June. $\bar{\zeta}_{2}$. Stem long; leav. very large; cor. Inng, dark purp.

2. A. Serpentaria : leaves cordate, oblong, acuminate; stem flexuous; peduncles radical; lip of the cerolla lanceolate. 
HAB. Shady woods. June. 4. Root fibr.; stem 8-10 in. high, genic.; fl. purp?

\section{DODECANDRIA.}

\section{ASARUM. Indian ginger.}

1. A. canadense: leaves broad-reniform, geminate; calyx woolly, deeply 3-parted; the segments sublanceolate, reflexed.

HAB. Shady rocks. May-June. 4. Leav. longpetiol., pubes. ; $f$. axill., pendul., solit.

2. A. virginicum: leaves solitary, roundish-cordate, smooth and coriaceous ; flower subsessile; calyx short-campanulate, smooth externally.

IIAB. Rocky woods. April. 4. Leas. very sinooth; seg. of cal. obtuse.

\section{MONOECIA.}

\section{MONANDRIA.}

524. ZOSTERA. Stam. and pist. inserted in 2 rows upon one side of a spadix. Spath foliaceous. STERIL: FL. Anth. ovate, sessile, alternating with the germens. FERTILE FL. Germ. ovate. Style bifirl. Drupe with 1 seed.

325. CAUlinia. sterile FL. Perianth 0 . .9nth. sessile. Fertile fl. Perianth 0 . Style filiform ; stigma bifid. Caps.1-seeded.

526. ZANNICHELLIA. Sterile Fl. Perianth 0. FERtile Fl. Cal. 1-leaved. Cor. 0. Style 1 : stigma peltate. Capsules about 4, sessile.

527. EUPHORBIA. Involucrum resembling a calyx, ventricose, the alternate segments (petals L. necturies?) petaloid. STERILE FL. 12 or more, mostly simple, each consisting of an anther with its filament articulated to a pedicel, (coming to maturity successively.) Cal, and Cor. very rarely present. FERTILE FL. solitary, central, stipitate. Cal. and Cor. 0. Styles 3, 2.cleft. raps. 3-lobed. 


\section{DIANDRIA.}

528. PODOSTEMUM. Sterile Fr. Perianth 0. Stam. 2, affixed to a common pedicel. FERTILE FL. Germ. ovate ; stig. 1, sessile. Caps. 2celled, 2-valved, many-seeded. Seeds minute.

\section{TRIANDRIA.}

529. TYPHA. Ament cylindrical, dense. STERILF FL. Perianth 0. Stam. 3 together, upon a chaffy or hairy receptacle, united below into 1 filament. FERTILe FL. below the sterile. Perianth o. Pericarp pedicellate, surrounded with a hairy pappus at the base.

530. SPARGANiUM. Ament globose. Sterile Fr. Cal. 3-leaved. Fertile Fu. Cal. 3-leaved. Drupe dry, 1-2-seeded.

531. CAREX. Flowers collected into an imbricate ament. Glume 1-flowered. Cor. ventricose, 1-valved, persistent, including the caryopsis.

532. SCLERIA. STERILE FL. Cal. 2- or 6-glumed, many-flowered. Valres of the Cor. unarmed Fertile fe. Cal. 2 or 6 glumed, 1 -flowered. Cor. 0. Stig. 1-3. Nut coloured, subglobose.

533. TRIPSACUM. STERILE FL. Glume 2-florvered; the exterior flower sterile, the interior neuter. Valve of the Cor. nembranaceous. Fertile FL. Glume 2-flowered; exterior valve resembling an involucrum, perforate near the base Cor. 2-valved. Styles 2. Seed 1.

i34. COMPTONIA. STERILF FL. Ament cylindric; scales 1-flowered. Cor. 0. Fertile fl. Amenl ovate. Cal. at length 6-leaved. Cor. 0. Styles 9. Nut oval, 1-celled.

\section{TETRANDRIA.}

835. ERIOCAULON. Florers collected into a $\mathrm{com}$. pact scaly head. STerile FL. in the disk. Perianth single, 4 -cleft; the 2 inner segments E c 
united nearly to their summit. Stam. 4-6. FERTILE FL. in the margin. Perianth single, deeply 4-parted. Style 1. Stig. 2-3. Caps. 2-3-lobed, 2-3-celled; cells 1-seeded.

336. ALNUS. STERILE FL. Ament composed of 3 . flowered, cuneiform truncate receptacles. Cal. the scales of the ament, 3-lobed. Cor. 4-parted. FERTILE FL. Scales of the ament 2-flowered, subtrifid. Cor. 0 . Seed compressed, wingless. 53\%. BOEHMERIA. sterile FL. Cal. 4-parted. Cor. 0. Vectary 0. FERTILE FL. Cal. and Cor. 0. Seed 1, compressed.

538. URTICA. Sterile Fl. Cal. 4-leaved. Cor. 0. Nectary (abortive germen?) central, mostly cyathiform. FERTILE FL. C'al. 2-leaved. $P e$. ricarp 1 seeded, shining.

539. PARIETARIA. Flowers polygamous. perfect. Cal. 4-cleft. Cor. 0. Stam. elastic. Style 1. Seed 1, superior. FERTILE. Cal. 2-leaved. Seed covered by the dry and elongated calyx.

540. MORUS. Sterile Fl. Cal. 4-parted. Cor. 0. FERTILE FL. Cal. 4-leaved, becoming a berry. Cor. 0. Styles 2. Seeds solitary.

\section{PENTANDRIA.}

541. CROTONOPSIS. STERILE FL. Cal. 5-parted. Cor. 5-petalled. FERTILE FL. Cal. 5-parted. Cor. 0. Stig. 3, twice bifid. Cap. 1-seeded 542. AMARANTHUS. Sterile FL. Cal. 3-5leaved. Cor. 0. Stam. 3-5. Fertile FL. Cal. 3-5 leaved. Cor. 0. Styles 3. Caps. 1celled, opening transversely all round. Seed 1. 543. XANTHIUM. Sterile Fu. Cal. imbricate. Anthers approximate, but not united. Recept. palaceaceous. FERTILE FL. Cal. a 2-leaved involucrum, 1-flowered. Cor. 0. Drupe? dry, muricate, 2-cleft. Nut 2-celled.

544. AMiBROSIA. Sterile Fl. Cal. 1-leaved. Anth. approximate, but not united. Recept. naked. FERTILE FL. Cal. 1-leaved, entire or 5-toothed, 1-flowered. Cor. 0. Nut formed from the indurated calys, 1 -seeded. 


\section{HEXANDRIA.}

545. ZIZANiA. sterile fl. Cal. 0. Cor. 2-valved, subawned. Fertile fl. Cal. 0. Cor. 2-valved, cucullate, awned. Style 2-parted. Seed 1, invested by the corolla.

546. HYYDROCHLOA. Sterile fl. Cal. 0. Cor. 2. valved, awnless. fertile fl. Cal.0. Cor. 2 valved, awnless. Stig. 2, very long. Seed 1, reniform.

\section{POLYANDRIA.}

$54 \%$ CERATOPHYLLUM. sterile Fl. Cal. manyparted. Cor. 0. Stam. 16-20; anth. tricuspidate. Fertilf Fl. Cal. many-parted. Cor. 0 . Stig. nearly sessile, oblique. Nut 1 -seerled. 518. MYRIOPHYLLUM. Sterile FL. Cal. 4-cleft. Petals 4, caducous. Stam. 4, 6, or 8 . Fertile FL. Cal.4-leaved. Petals 4. Stig. 4, pubescent; styles $0 . \quad \mathcal{N}$ uts 4 , subglobose, 1 -seeded.

549. SAGITTARIA. sterile Fr. Cal. 3-leared. Pet. 3. Stam. definite. Fertile Fl. Cal. 3leaved. Pet. 3. Germens numerous. Pericarps aggregated, 1 -seeded, not opening.

550. QUERCUS. STERILE FL. in a lonse ament. Cal. mostly 5-rleft. Cor. 0. Stam. 5-10. FERTILE FL. Involucrum cup-shaped, scaly. Cal. incorporated with the germen, 6-lobed. Germen 3-celled; 2 of the cells abortive. Style 1 ; stig. 3-5. Nut (acorn) 1-celled, coriaceous, 1 seeded, surrounded at the base by the enlarged cup-shaped involucrum.

551. CORYLUS. STERILE FL. in a cylindrical ament, its scales 3-cleft. Perianth 0. Stam. 3 ; anth. 1-celled. fertile, fi. Cal. obsolete. Gierm. several ; stig. 2. Nut ovate, surrounded with the enlarged coriaceous and scaly involucrum.

532. FAGUS. sterri.e FI. in a globose ament. Cal. 5-6-cleft, campanulate. Stam.5-12. FERTILE FL. 2. Involucrum 4-lobed, prickly. Cal. single, 4-5-cleft. Styles 3. Nut 1-seeded, in . closed in the enlarged echinate involucrum. 
353. CASTANEA. STERILE FL. in a long, naked cy lindrical ament. Cal. 1-leaved, 6-cleft. Stam. 10-12. FERTILE FL. 3. Involucrum 4-lobed, thickly muricate. Cal. 5-6-lobed. Styles 6. Nut mostly 1 -seeded, invested with the enlarged involucrum.

554. BETULA. STERILE FI. in a cylindrical ament; scales peltate, 3-flowered. Cal. 0. Cor. 0. Stam. 10-12. FERTILE FL. Scales of the ament 3-flowered. Cal. 0. Cor. 0. Styles 2. Nuts compressed, with a broad-winged margin, 1seeded.

555. CARPINUS. STERILE FL. in a cylindrical ament scales ciliate. Stam. about 10 . FERTILE FL. in a loose ament; scales large, 3-lobed, 1-2flowered. Cal. 3-cleft. Styles 2. Nut ovate. sulcate, 1 -seeded.

556. OSTRYA. STERILE FL. in a cylindrical ament; scales 1-flowered. Cal. 0. Filaments branched. FERTile Fl. Ament naked. Cal. 0. Cor. 0. Caps. inflated, imbricate, 1 -seeded at the base. 55\%. PLATANUS. Flowers in globose aments. STERILE. Cal. 0. Cor. minute. Anth. adnate to the filaments from the base. FERTILE. Cal. manyparted. Cor. 0. Stig. recurved. Caps. subclarate, 1-seeded, mucronate with the persistent style; base surrounded with a hairy pappus.

558. LIQUIDAMBAR. STERILE FL. in a conic ament, surrounded with a 4-leaved involucrum. Cal. and Cor. 0. Filam. numerous. STERILE FL. Ament globose, surrounded with a 4 -leaved involucrum. Cal. 1-leaved, urceolate, 2-flowered. Styles 2. Capsules 2, surrounded by the base of the calyx, 1-celled, many-speded.

559. JUGLANS. STERILE FL. Ament imbricate; the scales mostly 5-parted. Cal. about 5-parted. Stam. 20-30. Fentile Fl. Cal. 4-cleft, superior. Cor. 4-parted. Styles 1-2. Drupe partly spongy; nut rugose and irregularly furrowed.

560. CARYA. sterile Fl. Ament imbricate; scales 3-parted. Cal. and Cor.0. Stam. 4-6. FER- 
TILE Ft. Cal. 4-cleft, superior. Cor. 0. Styles 0 ; stig. subdiscoid, 4-lobed. Pericarp 4valred. Nut subquadrangular, even.

561. ARUM. Spathe 1-leaved, cucullate. Cal. and Cor. 0. Spadix naked above, bearing sessile anthers below the middle, and germens at the base. Berry 1-celled, many-seeded.

562. LECONTIA.* Spathe convolute. Cal. and Cor. 0. Spadix covered with flowers, fertile at the base, sterile above. Berry 1 -seeded.

563. CALLA. Spath ovate, somewhat flattened. Spadix covered with flowers ; barren and fertile inmixed. Cal. and Cor. 0. Berry many-seeded.

\section{MONADELPHIA.}

564. PiNUS. sterile fl. Scales peltate. Cal. and Cor. 0. Anth. 2, sessile, 1-celled. TERTILE $F L$. in an ovate or conical strobile; scales closely imbricate, 2-flowered. Cor. 0. Nut winged, covered by the scales of the cone.

505. THUYA. Sterile fl. Ament imbricate. Cal, and Cor. 0. Anth. sessile, 4. Fertile Fl. 0. Strobile with the scales 2-flowered. Cor. 0. Nut 1 , winged.

566. CUPRESSUS. STERILE FL. in an ovate ament; scales peltate. Cal. and Cor. 0. Anth. 4, sessile. FERTILE FL. Strobile with the scales peltate. Cal. and Cor. 0. Germens 4-8 under each scale of the strobile. Nuts angular, compressed.

567. ACALYPHA. Sterile Fl. Cal. 3-4-parted. Cor. 0. Stam. 8-16. Fertire Fl. Cal. 3leaved. Cor. 0. Styles 3. Caps. 3-celled. Seed 1 in each cell.

56s. PHYllanthuS. Sterile fl. Cal. 5-6parted. Filam. often columnar; anth. 3. FERTILF FL. resembling the sterile. Nectary a 12 . angled margin. Styles 3. Caps. mostly 3-grained. 569. Nelothria. Sterile fl. Cal. 1-leaved, 3-

*. A neir genus proposed by Mr. WV. Cooper, and named inbonour of Capt. Ieconte of the U. S. A.

E e 2 
5-toothed. Cor. campanulate. Stam. 3, growing to the tube of the corolla. FERTILE FL. Cal. and Cor. as in the sterile. Germen inferior; style 1; stig. 3. Berry 3-celled, manyseeded.

570. MOMORDICA. STERILE FL. Cal. 5-6-cleft. Cor. 5-6-parted. Filam. 3. FERTILE FL. Style 3-cleft. Pepo dry, bursting elastically.

571. SICYOS. Sterile Fl. Cal. 5-toothed. Cor. 5-parted. Filam. 3. Fertile Fu. Siyle 3 cleft. Pepo 1-seeded.

\section{MONOECIA.}

\section{MONANDRIA.}

524. ZOSTERA. Grass-wrack. Fluviales.

3. marina: leaves entire, somewhat 3-nerved; stem roundish.

HAB. Sea shore. Leav. linear, 3-4 lin. broad, $2-$ 4 ft. long; spad. lin., sheath.

525. CAULINIA. Fluviales.

1. C. flexilis: leaves verticillate by sixes, linear, denticulate at the extremity, spreading.

HAB. Flowing water. Aug.-Sept. 4 ? Stem submerg., branch.; $f$ l. axill., sess.

2. C. fragilis: leaves by threes, or opposite, linearsubulate, recurved, with aculeate dentures, rigid. HAB. Ditches, \&c. Aug. Stem long, brittle, sub. merg.

596. ZANNICHELLIA. Horned pondweed. Flu. viales.

1. Z. palustris : anther 4-celled; stigmas entire; pericarps toothed on the back.

HAB. Ditches, \&c. ๑. Stem long, filif.; leav. oppos., lin.: fl. axill., small.

2. Z. intermedia* : anther 2-celled; stigmas dentate crenate; seed smooth, entire on the back. 
IIAB. Salt marsh ditches. July. ○. Submerged; stem filif.; leav. entire; fl. inconspic.

527. EUPHORBIA. Spurge. Euphorbiacece.

1. E. hypericifolia: smooth, branched, erect-spreading; branches divaricate; leaves opposite, serrate, ovaloblong, subfalcate, on very short petioles; corymbs terminal and axillary.

IIAB. Fields and dry soils. July-Aug. ○. Very smooth, 12-18 in. high; leav. spot.

6? hirsuta: stem slender, hairy; leaves oblong, smooth, serrulate.

HAB. Sandy soils. Aug. Much branch., 3-12 in. high; stem purp.

3. E. maculata: stem procumbent, spreading flat on the ground, much branched, hairy ; leaves opposite, oval or oblong, serrulate, oblique at the base, on short petioles, smonth above, bairy and pale beneath ; flowers solitary, axillary, much shorter than the leaves.

HAB. Sandy fields. July-Oct. ๑. Stem 6-12 in. long; Al. appearing fascic.

3. E. polygonifolia: procumbent, divaricate, very smooth, succulent; leaves oblong and linearlanceolate, entire, obtuse at the base, sometimes subcordate; flowers solitary in the forks of the stem; stipules simple.

HAB. Sea shore. July-Sept. 4. Stem 8 in. long; stip. subul.; $f$. minute.

4. E. Ipecacuanha: procumbent or suberect, very smooth ; leaves opposite obovate and lanceolate; peduncles axillary, 1 -flowered, elongated.

HAB. Pine barrens. May-June. 4. Root tapering, very long; leav. variable, cllipt., obovate, or linear, (in young plants broad.)

5. E. corallata: umbel 5-cleft, 3-cleft, and dichotomous ; floral and cauline leaves oblong, obtuse, alternate dentures of the involucrum petaloid, obovate.

HAB. Fields, \&c. June. 4. Stem 1-2 $f t$ high, erect, subsimp.; fl. conspic.

i. E. portulaccoides: erect, leaves entire, oval, re- 
tuse ; peduncles axillary, 1-flowered, as long as the leaves.

HAB. Sandy soil. 4. A rariety of the preceding? \%. F. Peplus : umbel 3-cleft, dichotomous; flora] leares ovate, of the stem obovate, very entire, petiolate.

HAB. Sandy soil. July. ๑. Flor. leaves large; fl. conspic.

3. E. obtusata: umbel 3-rayed, twice dichotomous; floral leaves ovate, rather obtuse, subcordate; of the stem alternate, sessile, spathulate, serrulate, smooth ; capsule muricate.

HAB. Fields. July. 4. Stem 12-18 in. high, smooth, subsimple below.

9. E. mercurialina: stem slender and weak, simply 3cleft; leares opposite and ternate, subsessile, oval, entire; peduncles terminal, solitary, 1flowered.

HAB. Shady rocks. July-Aug. 4 .

10. E. pilosa: umbel of 5 primary rays and bifid ; floral leaves ovate; cauline lanceolate, somewhat hairy, serrulate at the extremity; petaloid segments of the involucrum entire.

IIAB. Woods, \&c. June-July. 4. Stem 1-i ft. high.

11. E. dentata: hairy; leaves opposite, oral, dentate: flowers crowded at the summit of the stem.

IIAB. Shady rocks. July-Aug. ๑ Upper leav. spotted.

32. E. helioscopia: umbel of 5 primary rays and bifid: floral leaves obovate; cauline cuneiform serrate. HАB. Rich soil. ๑. Stem erect; leav. scattered: caps. smooth.

\section{DIANDRIA.}

528. PODOSTEMUM. Fluviales.

P. ceratophyllum.

IIAB. Rocks, in streams. July. 4. Stem filif. Poat.; leav' pinnate; f. axill. 


\section{TRIANDRIA.}

529. TYFHA. Reed-mace. Aroidece.

1. T. latifolia : leaves linear; sterile and fertile spikes approximate on the same rachis, both cylindrical. IIAB Water. June-July. 4. Culm $5 \mathrm{ft}$. high; spikes dense; leav. 1 in broad.

๑. T. angustifolia: leaves linear, canaliculate, semicylindric below and flattish above; sterile and fer. tile spikes a little separate.

HAB. Water. June-July. 4. Culm 4-5 ft. high; leav. 5-6 lin. broad.

530. SPARGANIUM. Burr-reed. Aroidece.

1. S. ramosum: leaves triangular at the base, their sides concave; common flower stalk branched; stigma linear.

HAB. Borders of lakes, \&c. Aug.-Sept. 4. Tro ft. high; leav. ensif., long.

2. S. americanum: lower leaves equal with, or exceeding the stem, which is nearly simple; floral ones concave at the base and erect; stigma simple, ovate-oblong, oblique, scarcely more than half the length of the style.

IfAB. Läkes. Aug. 4. Stem $12 \mathrm{in.} \mathrm{high;} \mathrm{lower}$ leav. carinate; fertile heads $2-5$.

3. S. natans: leaves long, flat, floating; common flower-stalk simple; stigma ovate, very short ; head of sterile flowers subsolitary.

HAB. Lakes. Aug. 4. Stem long, slend.; leav. very narrow, linear, thin.

531. CAREX.a Sedge. Cyperoidece.

A. Inflorescence dioecious.

1. C. sterilis: spikes dioecious; sterile $3-5$; fertile about 6, (sometimes androgynous;) fruit ovate,

$a$ For full descriptions of the species enumerated below, sec the Monograph of North Amcrican Carices, by Rev. Mr. Schweinitz and myself, in the Annals of the Lyecum of Nat. History. 
compressed, triquetrous; margin ciliate-serrate, apez recurred and bicuspidate.

HAB. Marshes. May. 4. Culm 8-12 in. high; spik. subapprox., oblong.

B. Inflorescence monoecious.

* All the spikes androgynous.

$\dagger$ Spike solitary.

a. Summit staminiferous.

2. C. Fraseri: spike simple, ovate; fruit ovate-subglobose, entire at the point, longer than the oblong glume; leaves lanceolate, undulate, crenulate; scape sheathed at the base.

IAB. Mountains. April. \& Leav.broad, radi. cal.

3. C. Willdenovii: spike simple, ovate; sterile and fertile flowers about 6 ; fruit ovate, subglobose at the base, rostrate; glumes ovate, acuminate, the inferior ones very long and foliaceous, much exceeding the culm.

HAB. Rocky woods. May-June. \#. Culm a span high; inf. glum. 1-2 in. long.

4. C. polytrichoides : spike simple; fruit oblong-lanceolate, compressed triquetrous, obtuse, emarginate; glumes oblong-obtuse, mucronate.

HAB. Dry hills and bogs. May. 4. Culm foot high, very slend.; leav. narrow-linear.

5. C. pauciflora: spike about 4-flowered; sterile flower subsolitary, terminal; fruit lanceolate, terete, reflexed; fertile glumes caducous.

HAB. Sphagnous bogs. June. 4. A span high: fr. long., yell., caduc.

b. Summit pistilliferous.

6. C. squarrosa: spike mostly simple, (sometimes spikes $2-3$, ) very thick, oblong-cylindrical; fruit imbricate, at length horizontal, smooth, subsquarrose, bidentate at the point, longer than the lanceolate glume.

HAB. Bogs. June. 4. Culm $2 \mathrm{ft}$.high; spike 3 in. long, $\frac{1}{2}$ in. thick. 
* Spikes several, aggregated into a head.

7. C. cephalophora: spikes collected into an elliptical head; fruit ovate, scabrous on the margin above, about equal to the ovate subaristate glume.

HAB. Hills. May. 4. Culm 1-2 fl. high, ces. pit., reiry; head subtrifid.

** Spikes distinct, (not aggregated into a head.)

a. Summit staminiferous.

\section{With 2 stigmas.}

3. C. bromoides: spikes $4-6$, alternate, oblong, erect, uppermost one sterile above, the rest pistilliferous or androgynous (sterile above and below ;) fruit erect, lanceolate acuminate, scabrous, nerved, bifid, longer than the ovate-lanceolate glume.

HAB. Bogs. May. 4. Culm 12-18 in. high, slend.; spik. subdist., $\frac{1}{2}$ in. long.

9. C. retroflexa: spikes about 4, subapproximate, ovate, the lowest one with a short bract; fruit ovate-lanceolate, bidentate, scabrous on the margin, spreading and reflexed, as long as the ovate acute glume.

HAB. Meadows, \&c. May. 4. Culm 8-12 in: high, subhexang.; spik. 3-4-5.

10. C. rosea: spikes 4-6, remote, about 9-flowered, the lowest one with a setaceous bract overtopping the culm; fruit ovate, acuminate, diverging and radiate, scabrous on the distinct margin, twice as long as the ovate obtuse glume.

HAB. Moist woods, \&c. May. 4. Culm $12 \mathrm{in.}$ high; spikes $1 \mathrm{in}$. distant, yell.-gr.

11. C. disperma: spikes about 3, rather remote, mostly 2-flowered, somewhat erect, the lowest one bracteate ; fruit ovate, rather obtuse, nerved, plano-convex, smooth, with a scabrous margin, entire at the point, twice as long as the ovate, obtuse submucronate glume.

HAB. Mountain woods. May-June. 4. Culin 6-12 in. high; fruit small.

19. C. Muhlenbergii: spikes about 5, ovate, crowded at the summit of the culm, bracteate at the base; 
fruit broad-ovate, compressed, nerved, bifid, somewhat diverging, scabrous on the margin, rather shorter than the ovate mucronate glume.

HAB. Woods. May. 4. Culm 1-2 ft. high; plant dark green.

13. C. stipata: spike compound, oblong; spikelets numerous (10-15,) oblong, aggregated, bracteate; bracts a little longer than the spikelets; fruit lanceolate, subterete and suooth below, spreading, bidentate at the point, which is scabrous, twice as long as the glumes.

HAB Swamps. April. 4. Culm 1-3 ft. high, thick, small; spik. crowded.

14. C. sparganioides : spikelets about 8 , many-flowered; upper ones approximate, lower ones subdis: tant, bracteate ; fruit ovate, compressed, acuminate, bifid, diverging, scabrous on the margin. twice as long as the ovate mucronate glume.

HAB. Meadows. May. 4. Culm $2 \mathrm{ft}$. high; 2-4 lowest spik. remote.

15. C. multiflora: spike oblong, decompound, bracteate, interrupted; spikelets glomerate, ovate-oblong, obtuse ; fruit ovate, acuminate, compressed, crowded, bifid, 3-nerved, serrulate on the margin, at length diverging, rather shorter than the ovate cuspidate glume.

HAB. Wet meadows. May. 4. Culm 2 ft. high, obtusely triang.; spike subpanic.

16. C. setacea : spike oblong, decompound, bracteate; spikelets glomerate, ovate, obtuse ; fruit ovate, acuminate, compressed, bifid, subdiverging, as long as the ovate-lanceolate awned glume.

HAB. Meadows. June-July. 4. Culm 1830 in. high, acutely triang., sulcate.

17. C. priniculata: spike rlecompound, paniculate, interrupted, the branches alternate and somewhat remote ; fruit ovate, acuminate, spreading, margined above, bifid.

HAB. Wet meadows. May. 4. Culm $18 \mathrm{in.}$ high; spikes not black in age.

18. C. teretiuscula: spike decompound or paniculate, dense, subacute, (often dioecious, at length brown,) spikelets with short bracts at the base ; fruit ovate, 
acuminate, somewhat gibbous at the base, bidentate, ciliate-serrulate on the margin.

HAB. Bogs. May. 4. Culm 18 in. high; spitie narrow; fr. and glume brown.

\section{With 3 stigmas.}

19. C. pedunculata: spikes about 4, on long peduncles, very remote; fruit obovate, triquetrous, obtuse, smooth, entire at the orifice; glumes ovate, mucronate, (purple and green.)

HAB. Rocky hills. May. 4. Culm filif., 6 in. high; pedunc. mostly radical.

20. C. ovata: spikes about 5 , pedunculate, ovate, pendulous ; fruit obovate, acute at each end, as long as the ovate acute glume.

HAB. Canada. 4. Spikes densely imbricate; glumes brown.

\section{Summit pistilliferous.}

1. With 2 stigmas.

21. C. Dereyana: spikes about 3 , sessile, loose, two of them approximate, the third distant, with long bracts at the base, (except the highest ;) fruit oblong-lanceolate, subcompressed, rostrate, bifid at the point; beak serrulate; culm flaccid.

HAB. Woods. June. 4. Culm 1-4 ft. high, subprccumb.; plant yell.-green.

22. C. loliacea : spikes about 4 , rather distant, few-flowered; fruit elliptical, obtuse, nerved, compressed, erect.

HAB. Swamps. June. 4. Culm 2 ft. high, slend.; spik. 3-5-fl.

23. C. trisperma: spikes 3 , remote, alternate, sessile, ovate, uppermost one without a bract; fruit oblong, acute (or short-rostrate) entire at the point, many-nerved, subscabrous above, somewhat di. verging, longer than the oblong acute hyaline glume.

HAB. Mountain bogs. June. 4. Culm $18 \mathrm{in.}$ high; filif., prostr.; plant pale green.

24. C. arida: spikes 8 , (large) subapproximate, dry; fruit elliptical, compressed, winged, terete in the 
middle, acuminate at each end, divergingly bifid; culm leafy.

HAB. Meadows. June. 4 . Culm 2-3 ft. high; leav. dark green; spik. gray.

จ5. C. lagopodioides: spikes numerous, $(10-16$,$) el-$ liptic, crowded ; bract beneath the lowest overtopping the culm; fruit lanceolate, acuminate, erect, bicuspidate, with a narrow serrulate margin, twice as long as the ovate-lanceolate glume.

HAB. Wet meadows. May. 4. Spikes large, at first subcylind.

26. C. scoparia: spikelets mostly 5, (sometimes 6 or 7 ,) ovate, sessile, approximate, aggregate, lowest one bracteate ; fruit ovate-lancenlate, margined, nerved, smooth, bicuspidate longer than the lanceolate acuminate glume.

HAB. Wet meadows. May. 4. Fruit not wing$e d$, tareny zchen mature.

27. C. straminea: spikes about 5, (4-7,) roundish, approximate, with short bracts at the base; fruit roundish-ovate, rostrate, compressed, broadlyovate, bidentate, serrulate, longer than the lanceolate glume.

HAB. Wet meadows. May. 4. A foot high; spikes yellozish; fr. acuminate.

28. C. fonea: spikes numerous, $(8-10$,$) inferior$ ones distinct, upper ones aggregated and confluent; fruit ovate, acuminated, winged, bidentate, somewhat longer than the ovate glume.

HAB. Marshes. June. 4. Culm obtusely triang.; spik. subglob.; glumes rigid.

29. C. cristata: spikes numerous, $(8-15$,$) aggregated$ into a kind of head; fruit ovate-lanceolate, winged, diverging, serrate, longer than the orate-lanceolate glume.

HAB. Wet thickets. June. 4. Spike crozided into an ovate head.

30. C. festucacea : spikes obovale, $(5-8$,$) subap-$ proximate, bracteate; fruit roundish-ovate, rostrate, bidentate, winged, serrulate on the margin, longer than the ovate-lanceolate glume.

HAB. Woods and mendows. May. 4. Fruit green or silucr-gray, not becoming latuny. 
31. C. stellulata: spikes $3-4$, rather remote, upper one attenuate at the base, the rest ovate; fruit ovate, plano-convex, spreading, and at length reflexed, short-acuminate, scabrous on the margin.

HAB. Wet places. May. 4. Culm 8-18 in. high; fr. broad; point subentire.

32. C. scirpoides: spikes 4 , ovate, obtuse, approximate, uppermost one clavate; fruit ovate, bidentate, plano-convex, erect, and a little spreading, but not reflexed, subcordate, serrulate, longer than the ovate obtuse glume.

HAB. Meadows. May. 4. Culm 6-12 in. high, strict; fr. not reflexed.

33. C. curta: spikes about 6 , subrenote, somewhat cylindric-ovate, tumid, sessile ; fruit short-ovate, plano-convex, rather acute, erect, entire at the point.

HAB. Wet meadows. May. 4. Culm nodding at the end; glume rohitish.

34. C. tenera: spikes about 5 , obovate, rather remote, sessile, attenuate at the base, the lowest bracteate; fruit ovate, compressed, rostrate, serrulate, longer than the oblong-lanceolate glume.

HAB. Moist meadows. May. 4. Culm 15-30 in. high; spik. brownish. (Is it C. sterilis?)

35. C. remota : spikes alternate, remote; bracts leafy, very long; fruit ovate acuminate, bifid, somewhat compressed.

HAB. Woods. Pennsylvania?

2. With 3 stigmas.

36. C. atrata: androgynous spikes 3, pedunculate, crowded, subpendulous in fruit, (black;) fruit roundish-ovate, with a short beak, bidentate.

IIAB. High mountains. June. Culm 6 in. high ; spik. large, black.

**** Terminal spikes androgynous; the rest pistilliferous; stigmas 3.

3i. C. virescens : spikes 3, oblong, erect; upper one pedunculate, sterile below, the rest fertile, subsessile and bracteate; fruit ovate, obtuse, costate, jubescent. 
HAB. Dry woods. May. Culın 1-2 ft. higk; leav. and sheath pubes.; plant dull green.

ß. costata: spikes larger; fruit strongly costate; exterior sheaths purple.

HAB. Rocky hills. Culm $2-3 \mathrm{ft}$. high.

38. C. hirsuta: spikes 3, erect, approximate, densely fruited, upper one ovate-oblong, on a short peduncle; the rest ovate, subsessile, bracteate; fruit roundish-ovate, nerved, obluse, smooth, orifice entire, longer than the ovate acuminate glumes; leares and sheaths pubescent.

HAb. Rocky woods. May. Culm 12-18 in. high; spik. thick; young fr. pubes.

39. C. Buxbaumii: spikes about 4, obovate, subremote, upper one androgynous and pedunculate, the rest sessile, with very long bracts ; fruit ovate, obtuse, rather compressed, orifice entire, shorter than the ovate acuminate (brown) glume.

HAB. Sphagnous swamps. June. Culm $2 \mathrm{ft}$. high; glume dark br.; fr. green.

40. C. digitalis: spikes mostly 4, distant, slender, pedunculate, lonsely-llowered, nodding; uppermost androgynous, fertile above; the rest all fertile; fruit oblong, subtriquetrous, obtuse, smooth, longer than the oblong mucronate glume.

HAB. Meadows. May. Culm 18 in. high, slend.; spik. filif.; plant pale green.

11. C. formosa: spikes 4, oblong, thick, distant, on exsert peduncles, nodding, uppermost one sterile at the base ; fruit oblong, triquetrous, somewhat inflated, rather acute at each end; orifice nearly entire or 2-lobed, obscurely nerved, twice as long as the ovate acute glume.

HAB. Meadows. Culm 12-18 in. high; leav. often subpubes.; plant yellowish-green.

12. C. Torreyana: spikes 4, filiform, pedunculate, somewhat nodding, uppermost one sterile at the base; fruit oblong, triquetrous, acute at each end, slightly 2-lobed, shorter than the oblong aroned glume; leaves and sheaths pubescent.

HAB. Meadows. Culm 18 in. high, leafy; spik. slend.; rachis flex.; glum. hyaline. 
**** Staminiferous and pistilliferous spikes distinct. $\dagger$ Staminiferous spike solitary.

2. With 2 stigmas.

13. C. novoe anglice: sterile spike on a short peduncle; fertile $2-3$, sessile, ovate, few-flowered, rather remote; fruit oval-subtriquetrous, rostrate, minutely pubescent, longer than the ovate-mucronate glume; culm slender, subdecumbent.

HAB. High mountains. Culm 6-8 in. high; leav. sm.; spik. mostly 4-fl., loreest dist.

44. C. aurea: fertile spikes mostly 3 , oblong, looseflowered, subpendulous, rather approximate, lower ones pedunculate; fruit obovate or pyriform, obtuse, nerved, entire at the orifice, longer than the ovate acute glume.

IIAB. Wet rocks. May-June. Culm 3-10 in. high, subprocum.; fruit dark orunge.

2. With 3 stigmas.

\$ Pistilliferous spikes sessile, or with the peduncles inclosed.

15. C. varia : fertile spikes $2-3$, approximate, fewflowered, ovate, subsessile; sterile spike sessile, (or on a short peduncle;) fruit subglobose, acuminate, bitid, obtusely triangular, hispidly pubescent, as long as the ovate acuminate glume.

HAB. Rocky woods. April. Culm 8-12 in. high, filif.: fr. ventricose.

46. C. marginata: sterile spike pedunculate; fertile spikes mostly 2 , approximate, subglobose, subsessile ; fruit globose, woolly, bidentate, longer than the ovate-oblong glume.

IIAB. Dry woods. April. Culm s-12. in. high ; sterile spike subtrigon.; glume margined.

17. C. vestita: sterile spike mostly solitary (rarely geminate, with the upper one elongate,) pedunculite, cylindrical-oblong; fertile 2, ovate-oblong, sessile, subapproximate, sometimes sterile at the summit ; fruit ovate, subtriquetrous, nerved, with a short rostrum, pubescent, rather longer than the ovate mucronate glume.

F f 2 
HAB. Wet sandy soil. June. Culm $2 \mathrm{ft}$. high; glum. brown; fr. bidentate.

18. C. pubescens: sterile spike subsessile; fertile 3, oblong, erect, rather loosely-flowered, the lowest on a short peduncle; fruit ovate triquetrous, rostrate, pubescent, orifice vearly entire, as lung as the ovate mucronate glume; leaves and culm pubescent.

HAB. Woods. May. Culm 12-18 in. high, erest or subdecum.; leav. pale gr.

19. C. flava: sterile spike on a short peduncle; fertile mostly 3, ovate, subapproximate, (the lowest rather remote, on short includer peduncles; fruit ovate, densely imbricate, bidentate, with a curved and reflexed rostrum, shorter than the ovate-lanceolate glune.

HAB. Highland mearlows. Culm 12-14 in. high, (sometimes 3-1;) plunt yellowish.

50. C. Ederi : sterile spike on a short peduncle ; fertile about 3 , ovate, approximate, subpedunculate, densely flowered ; fruit ovate-globose, horizontal, with a straight rostrum.

$\mathrm{H} \Lambda \mathrm{B}$. Wet rocks and banks. June. Culn 6-12 in. high; spik. and fr. small, yell.

51. C. tentaculata: fertile spikes 2-3, (rarely 4,) sessile, ovate or ovate-cylindrical, approximate, horizontal; bracts very long; fruit ovate, ventricose, nerved, with a very long rostrum, orifice bidentate, longer than the lanceolate glume.

11AB. Wet meadows. May-June. Culn 12-18 in. high; spik. very large, thick.

5\%. C. nigra: sterile spike pedunculate; fertile $2-3$, (rarely 4 ,) rather remote, oblong (black,) sessile, erect ; fruit obovate, compressed-triquetrous, subacute, orifice entire, as long as the ovate glume.

HAB. High mountains. July. Culm 3-12 in. high; fr. smooth, nerveless; styles $2-3$.

53. C. lupulina: sterile spike on a short peduncle, (rarely geminate;) fertile 3 , subsessile, ovate-oblong, erect, approximate; bracts very long and leafy ; fruit ovate, inflexed, nerved, long-rostrate, bicuspidate, much longer than the ovate glume. 
H.1B. Swamps. June. Culm 2-3 ft. high, thick and leafy; spike very thick.

B. polystachia: fertile spikes 5, oblong-cylindric, lowest one remote, on a lorig peduncle.

HAB. Swamps. Culm 2 ft. high; spik. 2-3 is. long.

54. C. folliculata: sterile spike pedunculate; fertile 2 , (often solitary,) roundish, approximate, fewflowered, upper one sessile, lower one short peduncle; bracts leafy ; fruit ovate, acuminaterostrate, ovate, reflexed and diverging, bicuspidate.

HAB. Swamps. June. Culın $18 \mathrm{in.} \mathrm{high;} \mathrm{spik.}$ $6-10-f l . ; p l$. dark green.

55. C. Xanthophysu: fertile spikes 3-4, ovate, very remote, pedunculate, few-flowered; fruit oblongconical, somewhat inflated, striate, horizontal when mature, acute, bifid, longer than the ovate acuminate glume.

HAB. Swamps. June. Culm 2-4 ft. high, slend.; $f r . \frac{1}{2}$ in. long ; pl. yell.-gr.

56. C. subulata: sterile epikes short-pedunculate; fertile mostly 4, sessile, or with included peduncles, very remote, few-flowered, sterile at the apex; fruit subulate, reflexed, much longer than the lanceolate glume; culm very slender.

HAB. Cedar swamps. July. Culm $2 \mathrm{ft}$. high; spik. about 4-fl.

57. C. alpestris : fertile spikes 3, 5-flowered, the 2 uppermost approximate and sessile, the lowest radical, on a long peduncle ; fruit obovate-oblong, triquetrous, scarcely rostrate, subpubescent, oritice oblique, as long as the oblong glume.

HAB. IVoody hills. Culm 6 in. high.

ఏ. Pistilliferous spikes on exsert peduncles, parlly sheathed at the base.

5\%. C. alba: sterile spike pedunculate; fertile 2-3, pedunculate, about 5-flowered; fruit obovate, with a short rostrum, obliquely truncate; sheath at the base of the culm hyaline, leafless.

HAB. Limestone hills. June. Culn 4-10 in. high, slend.; bracts wth.; fr. dark. 
50. C. plantaginea : fertile spikes mostly 4 , on pedun. cles scarcely excerted, loosely-flowered; fruit cblong-cuneiform, triquetrous, recurved at the apex; culm sheathed at the base; sheaths of the culn all lenfless, (coloured;) leaves broad.

HAB. Mountain woods. April-May. Culn 812 in. high, leafless; sheath purp.

60. C. anceps: fertile spikes mostly 3, remote, subcylindric, loosely flowered, lower ones pedunculate ; fruit ovate, triangular, acute, striate, narrowed at the base, orifice obscurely bidentate, about as long as the ovate cuspidate glume.

HAB. Woods. April-May. Culm 12-14 in. high; hyemal leav. very broad.

61. C. oligocarpa: fertile spikes 3 , pedunculate, fewflowered; lower peduncles elongate ; fruit shortovate, acutely triangular, with a short rostrum, oritice entire, longer than the orate glume.

HAB. Rocky woods. May. Culm 6 in. high, slend. ; leav. subg!auc. ; spik. 5-8-fl.

62. C. scabrata: fertile spikes 5, subremote, cylindrical, mostly erect, lower ones long-pedunculate ; fruit ovate, with an acuminate rostrum, subventricose, scabrous, orifice oblique and somew bat bifid, longer than the ovate-lanceolate ciliate glume.

HAB. Bogs. May. Culm 18 in. high; leav. broad. very scab.; plant dark green.

63. C. conoidea: fertile spikes $2-3$, oblong, remote, rither loose, uppermost subsessile, lower ones ou long peduncles; fruit oblong-conical, obtuse, recurved at the apex, as long as the awnerl glume.

IIAB. Woods and meadows. May. Culm G-12 in. high, leav. thin.; spik. pale gr.

i.1. C. tetanica: sterile spike long-pedunculate; fertile 2-3, remote, rather densely flowered, upper one subsessile, lowest on a long peduncle; fruit ovate-oblong, acute at each end, nerved, subgibbous at the summit, oblique, orifice entire, longer than the ovate mucronate glume.

HAB. Moist meadows. May. Culm 12 in. high; spikes very remote; fr. subterete.

1). (.. laxiflora: sterile spike subsessile ; fertile mostly 3 , rather loose, remote, pedunculate, erect; 
fruit ovate oblong, ventricose, obtuse, somewhat shining, longer than the ovate cuspidate glume.

HAB. Woods and meadows. May. Culm 12$18 \mathrm{in.} \mathrm{high;} \mathrm{fr.} \mathrm{inflated} \mathrm{when} \mathrm{mature.}$

66. C. granularis: sterile sessile or short-pedunculate; fertile mostly 3 , remote, cylindrical, dense ; uppermost subsessile, lowest on a long peduncle ; fruit globose-ovate, nerved, orifice entire ; rostrum very short and recurved.

HAB. Wet meadows. May. Culm 12 in. high; leuv. subglauc.; spikes cylind., thick.

67. C. sylvatica: fertile spikes mostly 4 , remote, filiform, dense, peduncles nodding; fruit orate, rostrate, bifid, twice as long as the ovate mucronate glume.

HAB. Woods. May. Culm 12-18 in. high; spik. $1 \frac{1}{2}$ in. long; plant pale gr.

63. C. flexuosa; fertile spikes 4, remote, filiform, on nodding peduncles; fruit distant, alternate, oblong, acute at each end, rostrate, bifid, twice as long as the ovate-mucronate glume.

HAB. Meadows. June. Culm 18-24 in. high, spik. 2 in. long; rach. flex.

$\S \S \S$ Pistilliferous spikes on long peduncles, nearly destitute of sheaths.

69. C. umbellata: cespitose; fertile spikes mostly 4, orate, ferw-thowered, one sessile at the summit of the culm, the rest on radical pecluncles and appearing subumbellate; fruit ovate, acuminate-rostrate, subpubescent, as long as the ovate acuminate glume.

HAB. Rocky hills. April. Culm 1-6 in. high; leav. long; spik. $3-5,6-8-8$.

70. C. miliacea : fertile spikes 3 , slender and cylindrical, nodding, slender and filiform; fruit ovate, triangular, without nerves, slightly rostrate, orifice entire, as long as the ovate-lanceolate glume.

HAB. Moist meadows. Culm 12-15 in. high; spik. subapprox., 1-2 in. long, $g r$.

71. C. pullescens : fertile spikes 2-3, ovate-cylindrical, dense, at length somewhat nodding; fruit obovate-oblong, obtuse; sheaths and culm pubes. cent. 
HAB. Wet meadows. May. Culm $12 \mathrm{ir.} \mathrm{high;}$ fert. spik. mostly 3, thick, pale gr.

72. C. hystericina: sterile spike pedunculate; fertile $2-3$, thick, at length cernuous, upper one inclusely pedunculate, the rest on exsert peduncles; fruit ovate, inflated, subhorizontal, many-nerved, rostrate, oritice bifid, twice as long as the oblong awned glume.

HAB. Wet meadows. May. Culm 12-18 in. high; fert. spik. 1 in. long; plant yell.-gr.

73. C. Pseudo-cyperus: fertile spikes 4, cylindrical, pedunculate, upper ones subgeminate; fruit ob long-lanceolate, rostrate, reflexed, many-nerved, apex divaricately bifid.

IIAB. Swamps. June. Culm $2-3 f t$. high, thick: spik. 2 in. long, dense.

74. C. limosa: fertile spikes mostly 2 , ovate or oblong-ovate, pedunculate, somewhat distant, pendulous ; fruit suborbicular-elliptic, compressed, with a very short rostrum, (green,) orifice entire, as long as the ovate mucronate glume.

HAB. Sphagnous swamps. June. Culm 9-15 in. high; glum. variable, ovate, oblong, acum. or cuspid, often very long.

t $\dagger$ Staminiferous spikes 2 or more.

1. With 2 stiginas.

75. C. cespitosa: sterile spike subsolitary (or geminate ;) fertile mostly 3 , cylindrical, obtuse, distant, the lower cn a short exsert peduncle; bracts strict; fruit ovate, somewhat acute, densely fruited in about $\&$ rows, orifice minute; longer than the ovate (black and margined) glume; leaves spreading.

IIAB. Mountain bogs. Culm 12-18 in. high; leav. dark gr.; fr. gr. nerveless.

76. C. crinita: sterile spikes geminate, (sometimes androgynous ;) fertile 4 , distant, pendulous, cylindrical, deuse; fruit roundish-ovate, ventricose, slightly rostrate, orifice entire, much shorter than the lis.ear glume. 
HAB. Swamps. June. Culn 2-4ft. high; leav. pale gr.; spik. 2-3 in. long.

2. gynandra: fertile spikes 3, oblong-cylindrical; fruit short-ovate, somewhat longer than the awned glume.

HAB. Meadows. Culm 12-14 in. high; fr. yellowish when mature.

77. C. acuta: sterile spikes $1-3$; fertile mostly 3 , subpedunculate, somewhat nodding, cylindrical, remote; fruit oblong, entire at the orifice, as long as the oblong acute glume.

HAB. Wet meadows. Culm $2 \mathrm{ft}$. high, sharply triquetr.; glume br.; fr. nerveless.

\section{With 3 stigmas.}

78. C. Barraitii: sterile spikes subgeminate; fertile about 3, oblong-cylindrical, sterile at the summit, nodding, distant ; fruit oblong, subtriquetrous, somewhat scabrous, orifice subentire; a little shorter than the ovate lanceolate glumes; leaves glaucous.

HAB. Sea coast. Culm $12 \mathrm{in.} \mathrm{high;} \mathrm{leav.} \mathrm{very}$ smooth; glum. dark brown.

73. C. trichocarpa: sterile spikes 2-4, pedunculate, (sometimes sterile at the summit;) fertile 3 , distant, pedunculate, erect, oblong-cylindrical ; fruit ovite-lanceolate, acuminate, bicuspidate, hairy, longer than the ovite acuminate glume.

HAB. Swamps. Culm 3 ft. high; fertile spik. thick, subcylindr.; pedunc. appress.

80. C. filiformis: sterile spike geminate; fertile 2 , ovate-oblong, sessile, distant ; fruit elliptical, rillous, bifurcate, as long is the ovate-lariceolate somewhat awned glume; leaves convolute.

HAB. Bogs. Culm 2-3 fl. high, slend.; rad. leav. 2-3 ft. lons, filif. abore.

\$1. C. vesicuria: sterile spikes 3 ; fertile mostly 2 , pedunculate, cylindrical ; fruit oblong, inflated, acnminate-rostrate, bicuspidnte, longer than the linceolate glume; culm acutely triquetrous.

11AB. Wet meadows. May. Culm 2 ft. high; spik. dense, $2--3$ in. long.

32. C. ampullacea: sterile spikes 3 ; fertile $:-3$, 
cylindrical, short pedunculate, erect; fruit sub. globose, inflated, rostrate, bifurcate, longer than the lanceolate glume; culm obtusely triangular.

HAB. Wet meadows. In N. America?

33. C. retrorsa: sterile spikes about 3 , lower one often fertile at the base ; fertile spikes about 5 , approximate, (and clustered in a subcorymbose manner,) oblong-cylindrical, inclusely pedunculate, lowest one often remote; fruit ovate, inflated, reflexed, rostrate, half as long as the lanceolate glume.

HAB. Borlers of ponds. Culm $2 \mathrm{ft}$. high; fert. spik. thick, dense.

84. C. Schweinitzii : sterile spikes 2, upper one elongate, pedunculate; fertile 3 , oblong-cylindrical, subpendulous, rather remote, inclusely pedunculate; fruit oblong-ovate, acuminate-rostrate, inflated, bifurcate, longer than the lanceolate attenuate glume.

HAB. Wet sandy soil. June. Culm 12 in. high; spik. light straw-colour.

\$5. C. bullata: sterile spikes 3 ; fertile 2, oblongcylindrical, rather loose, exsertly pedunculate and somewhat nodding, distant ; fruit ovate-globose, inflated, erect, smooth, costate, rostrate-acuminate, orifice bifid, twice as long as the lanceolate glume.

IIAB. Bogs. Culm 18-24 in. high, slend.; fert. spik. remote, fere-fl.

¿6. C. pellita: sterile spikes 2 , oblong; fertile 2 , cylindrical, remote, erect, upper one sessile; fruit ovate, subtriquetrous, short-rostrate, hairy, bicuspidate, equal to the oblong awned glume.

HAB. Wet meadows. May. Culm $2 \frac{1}{2} \mathrm{ft}$. high; leav. subrigid, flat.

37. C. lacustris: sterile spikes about 4 ; fertile $2-3$, erect, oblong-cylindrical, short pedunculate ; fruit oblong, many-nerved, subrostrate, smooth, bifurcate, somewhat longer than the oblong mucronate glume.

HAB. Deep swamps. June. Culn $3-5$ ft. high; leav. broad; fr. brown.

38. C. longirostris: sterile spikes 3 , short; fertile $2--$ 
3 , cylindrical, loose, at length pendulous, longpedunculate, subdistant ; fruit ovate, subglobose at the base, smooth, bifid, rostrum very long, longer than the lanceolate glume.

HAB. Meadows and rocky woods. Culm $2 \mathrm{ft}$. high, slend.; pedunc. filif.

\section{SCLERIA. Whip grass. Cyperacece.}

1. S. triglomerata: culm erect, acutely triquetrous, scabrous; leaves broad-linear, subscabrous and a little hairy ; spikes lateral and terminal, fascicu. late; glumes ciliate, mucronate; nuts smooth and polished.

HAB. Marshes. July. 4. Culm 3-4ft.high; nut large, white, hard.

2. S. pauciflora: stem triquetrous and with the narrow leaves smooth; spikes lateral and terminal, few-flowered, the lateral ones pendulous, terminal, fasciculate; glumes smooth ; seed roughened.

HAB. Wet meadows. Aug. H. Culm $2 \mathrm{ft}$. high, slend.; lat. spikes 2.

3. S. verticillata: stem simple, triquetrous, and with the leaves smooth; spike glomerate, naked, the clusters alternate, distant; glumes smooth; seed globose, mucronate, transversely corrugate.

HAB. Mearlows. Aug. H. Culm $1 \mathrm{ft}$. high, slcrd.; nut small, tuberculate.

533. TRIPSACUM. Sesame grass. Graminex.

' $\mathrm{A}$. dactyloides: spikes numerous, (3-4,) aggregate; florets sterile near the summit, fertile at the base. HAB. Dry hills. Aug. 4. Culm 5-7ft. high: spik. large; stig. long, purp.

3. monostachyon: spike solitary.

IIAB. With the preceding.

534. COMPTONIA. Sweet fern.

C. asplenifolia.

HAB. Woods. May. Shrub $2-3$ ft. high, aro. matic; leav. lobed-pinnat.

G g 


\section{'TE'TRANDRIA.}

\section{ERIOCAULON. Pipewort. Restiacece.}

1. E. pellucidum: scape slender, about 7-furrowed; leaves linear-subulate, canaliculate, smooth, pellucid, 5-nerved, transversely striate; head small, globose; scales of the involucrum oval, obtuse.

HAB. Water. June. 4. Scape 4-6 in. high; leav. rad., cesp.; fl. white.

2. E. decangulare: scape 10-furrowed; leaves ensiform, smooth; head large, hemispherical ; scales of the involucrum oval, acute; of the receptacle mucronate.

HAB. Water. Aug. Scape 2-4 ft. high; leav. without millrib; fl. wh.

\section{ALNUS. Alder. Salicince.}

1. A. serrulata: leaves obovate, acuminate, with the veins and their axils hairy underneath; stipules elliptical, obtuse.

HAB. Low grounds. March-Apr. Shrub 8-15 ft. high, much branch.

2. A. undulatu: leaves oblong, acute, rounded at the base, doubly serrate; petioles and the veins beneath Lairy; axils of the reins naked; stipules ovate-oblong.

HAB. Mountain swamps. April. Shrub 3-4 ft. high.

\section{BOEHMERIA. Urticco.}

1. B. cylindrica: leaves opposite, ovate-oblong, acuminate, dentate, smooth; flowers diøecious; sterile spikes glomerate, interrupted; fertile cylindrical; stem herbaceous.

HAB. Shady swamps. July-Aug. 4. Stem slend., 2 ft. high; leav. petiol.; fl. minute.

2. B. lateriflora: leaves alternate, ovate-lanceolate, acuminate, serrate, scabrous; stem herbaceous. HAB. Woods. July. 4. Stem smooth; lear. scabrous on both surfaces. 


\section{URTICA. Nettle. Urticex.}

1. U.pumila: leaves opposite, ovate, acuminate, 3nerved, serrate; inferior petioles as long as the leaves; flowers monoecious, triandrous, in corymbed heads, shorter than the potioles.

HAB. Wet places. July-Aug. ๑. Stem succulent, semitransparent; leav. shining.

2. U. urens: leaves opposite, elliptical, about 5-nerved, acutely serrate; spikes glomerate, by pairs.

HAB. Waste places. May. ๑. Stem 2 ft. high; plant stinging. $\hat{\jmath}$.

3. U. dioica: leaves opposite, cordate, ovate-lanceolate, coarsely serrate; flowers diœecious; spikes paniculate, clustered, by pairs, longer than the. petiole.

HAB. Roadsides, sc. May. 4. Stem 2-3 ft. high, erect; fl. sm., green; plant stinging. $\hat{\oint}$.

4. U. procera : leaves opposite, ovate-lan eolate, serrate; petioles cilinte ; flower; diœcinus; spikes somewhat branched, clustered, by pairs, longer than the petioles.

HAB. Shady banks. July-Aug. 4. Stem 3-4 ft. high; leav. subacuminate.

5. U. capitata: leaves alternate, cordale-ovate, acuminute, serrate, 3-nerved, twice as long as the petinle ; clusters spikerl ; spikes solitary, shorter than the leaves, leafy at the summit ; tem naked.

HAP. Shady woods. June-July. 4. Stem 4$5 \mathrm{ft}$. high; leav. scab., on the stem opp ssite.

i. U. divaricata: leaves alternate, ovate, acuminate. rather smooth; petioles long, ciliate; panicles axillary, solitary, much branched an divaricate, longer than the petiole; stem stingin:

HAB. Damp rocky places. July-A 2-3 ft. high, branch.; leav. not cordrite.

7. U. canudensis: leaves cordate-orate, acuminateserrate, hispid on both sides; panicles axillary, generally by pairs, much branched and divaricate, inferior ones sterile and longer than the petiole, upper ones fertile, elongate, stem vory hispid stinging. 
HAB, Shady rocks. Aug.-Sept. 4. Stem 4-$6 \mathrm{ft}$. high; fibres tough.

539. PARIETARIA. Pellitory. Urticece:

P. pennsylvanica: leaves oblong-lanceolate, veined, with opake dots; involucrum 3-leaved, longer than the flower.

HAB. Rocks. July. ๑. Stern slend. 6-12 in. nigh, simple; fl. axill., clustered.

540. MORUS. Mulberry. Urticea.

II. rubra: dicecious; leaves cordate, ovate, acuminate, often 3-lobed, equally serrate, scabrous, pubesa cent beneath; fertile aments cylindrical.

HAB. Woods. May. A large tree, with long branches; leav. in young trees divided.

\section{PENTANDRIA.}

541. CROTONOPSIS. Euphorbiacece.

C. linearis: stem erect, dichotomously branching; leaves stellately pubescent above, covered with silvery scales beneath.

HAB. Swamps in pire barrens. June. 4. Stem 12-1.8 in. high: leav. lanc. or ovate.

\section{AMARANTHUS. Amaranthacea.}

1. A. lividus: flowers clustered, triandrous, in rounded spikes; leaves elliptic, retuse ; stem erect.

HAB. In cultivated grounds. Aug. ๑. Slem 2 fi. high, smooth.

3. A. hybridus: flowers pentandrous; racemes decompound, erect, crowded; leaves ovate-lanceolate.

HAB. Waste grounds. Aug. ๑. Stem 2-3 ft. high; racem. large, thick. §.

3. A. Blitum: flowers triandrous ; racemes somewhat spiked; flowers 3-leared; leaves ovate, retuse : stem spreading.

HAB. Waste places. July. ๑ Stem prostr., branched. $\delta$. 
4. A. spinosus: flowers pentandrous, in terminal compound racemes; axils spiny.

HAB. Waste grounds. June-Aug. ๑. Stem branch., diffuse, mostly coloured. §.

5. A. pumilus: flowers pentandrons, in axillary clusters; leaves ovate, obtuse, emarginate, fleshy, subrugose; stem diffuse, smooth.

HAB. Sandy sea coast. Aug. ๑. Stem and leav. purplish; plant smooth.

543. XANTHIUM. Clot-weed. Urticece.

1. X. strumarium: stem unarmel, branching; leaves cordate, lobed, unequally serrate, scabrous, 3nerved; fruit elliptical, armerl with uncinate rigid bristles; horns straight, spreading.

IIAB. Old fields, \&c. Sept. ๑. Stem 2-3 ft. high; leav. lavge; $f$. axill.

3. X. macrocarpon: stem unarmed, spotted; leaves cordate, lobed, obscurely sinuate-dentate, scabrous, 3-nerved; fruit oval, densely armed with short rigid uncinate bristles; horns incurved.

HAB. Near salt water. Aug. ○. Stem purp.spotted; fr. very large, woolly.

3. X. spinosum: spines ternate; leaves 3 -lobed.

HAB. Waste places. Aug. ๑. Stem $3 \mathrm{ft}$. high, much branch. ; spin. long, yell.; fr. small.

5.44. AllBROSIA. Hog-weed. Urticea.

1. A. trifida: hairy, rough; leaves 3-lobed, serrate, with oval-lanceolate acuminate lobes; fruit 6 spined below the summit.

IIAB. Along ditches, \&c. Sept. ๑. Stem 4-6 ft. high; leav. very large; spik. long, axill. and term.

2. A. elatior: leaves bipinnatifid, nearly smooth; petioles ciliate; racemes terminal, paniculate; stem virgate.

IIAB. Fields. Sept. ๑. Stem 1-3 ft. high, pubes. when young; seg. of leav. acute.

3. A. artemisifolia: leaves bipinnatifid, hoary beneath, the uppermost pinnatifid; racemes by threes, ter minal; branches fastigiate.

$\mathrm{G} g$ 2 
HAB. Fields. Aug.-Sept. ๑. Stem 4 ft. high : leav. subpubes. above, whitish beneath.

4. A. heterophylla: leaves of the stem pinnatifid, subdentate, petiolate; uppermost ones lanceolate, sessile; petioles conspicuously ciliate; racemes terminal, solitary ; stem paniculate.

HAB. Banks of rivers and ditches. Aug.-Sept.

\section{HEXANDRIA.}

545. ZIZANIA. Wild rice. Graminece.

1. Z. aquatica: panicle pyramidal, divaricate and sterile at the base, spiked and fertile above; pedicels of the flower clavate; awns long; seed linear.

HAB. In water. Aug. 4. Culm 4-m fi. high, thick; leav. broad-lin.; fl. decid.

Q. Z. miliacea: panicle effuse, pyramidal ; glumes with short awns; sterile and fertile florets intermixed; style 1; seed ovate, smooth ; leaves glatcous.

HAB. In water. 4. Culm 6 ft. high; joints smooth; leav. broad, perennial.

546. HYDROCHLOA. Graminea.

H. fluitans.

HAB. Lakes. July. 4. Floating; culm long: slender; leav. flat; spik. solit., axill., setaceous: glumes atonless.

\section{POLYANDRIA.}

547. CERATOPHYLLUM. Hornwort. Uncertain.

C. demersum : fruit armed with 3 spikes.

HAB. Under water. July. 4. Stem long, slend, much branch.; leav. verticill.

548. MYRIOPHYLLUM. Water milfoil. Haloragece.

2. M. spicatum: sterile flowers in interrupted leafles: spiked whorls. 
HAB. In water. Aug.-Sept. 4. Stem slend., branch.; leav. verticil. by 4's, finely pectin.

2. M. verticillatum: leaves pinnate, capillary, upper ones pectinate-pinnatifid; flowers axillary: verticillate, upper ones sterile, octandrous.

HAB. In water. July. 4: Floating; upper $A$. sometimes perfect.

3. M. heterophyllum: inferior leaves capillary-pinnate, superior oval-lanceolate, acutely serrate; flowers: hexandrous, the highest often perfect.

IIAB. Lakes. July. ४. Floating; stem simp.; upper leav. in 5's and 6's; fl. purp.

4. M. capillaceum: leaves all capillarly-pinnate ; flowers axillary, opposite, and alternate, tetrandous, mostly perfect; fruit quadrangular; formerl of 4 cylindrical seeds.

HAB. Ponds. July-Aug. 4. Floating; stem branch., dichot.; leaves hair-like.

3. M. ambiguum: leaves petiolate, pinnate, the lowest capillary, emerging ones pectinate, uppermost nearly entire, subserrate ; flowers perfect; anthers partly oblong; fruit quadrangular.

HAB. Ponds. July. 4. Floating, dichot.; leav. atten. at base; $f$. axill. solit.

f. limosum : stem rosting, erect, or procumbent :leaves rigid, partly entire, or divided above, with setaceous divisions.

HAB. Miry shores. July. 4 . Stern $1-4 \mathrm{in}$. high; leav. spread.; seg. 3-5; anth. oblons.

3. M. tenellum: erect, nearly leafless; bracts entire, obtuse; petals linear, conduplicate and revolute; flowers mostly perfect, tetrandrous.

HAB. Borders of ponds. July. 4. Slem simp, 4-12 in. high; fl. alternate; pet. oblong-lin.

SAGITTARIA. Arrowhead. Jancea.

3. S. sagittifolia: leaves sagittate, acute; lobes acule, straight, lanceolate.

c. latifolia: leaves broad-ovate, rather obtuse; lobes ovate, slightly acuminate, straight.

3. hastata: leares oblong-lanceolate, acute; lobes 
spreading, lanceolate, long-acuminate; flowers mostly dinecious.

$\%$ gracilis: leaves linear; lobes much spreading; linear, very long and acute.

$\delta$ pubescens: leaves and stem pubescent; bracts and calyx very pubescent.

HAB. Ponds, \&c. July. 4. Leav. large; scapc 1-2 ft. long; $f$. large, wh., verticil. in 3's.

2. S. obtrısa: leaves sagittate, dilated-ovate, rounder at the extremity, mucronate; lobes approximate, oblong, obliquely acuminate, straight; flowers dioecious ; sterile scape branched at the base.

IIAB. Ponds. Juiy. 4. Leav. large; sap milku, affording gum; fl. wh.

3. S. heterophylla: leaves simple, linear and lanceolate, aciste at each extremity, or elliptical and sagittate, with the lobes linear and divaricate; scape simple, few-flowered; flowers monoecious: the fertile subsessile.

IIAB. Ponds. July. 4. Scape $1 \mathrm{ft}$. high; leav. rarely sagitt.

4. S. rigida: leaves narrow-lanceolate, carinate beneath, rigid, very acute at each extremity ; scape branched ; flowers monoecious.

IIAB. Deep water. July-Aug. 4. Very tall; petioles stiff; $f$. numerous.

5. S. simplex: leaves linear-lanceolate, acute, narrowed towards the base; scape simple, manyflowered; flowers dioecious.

HAB. Muddy banks, \&c. Aug. 4. Scape 8-15 in. high; leav. not rigid; $f$. small.

3. S. acutifolia: leaves subulate, sheathed at the base, convex on the back; scape simple, few-flowered, longer than the leaveg; flowers monoecious.

HAB. Muddy shores. Aug. 4. Scape 6 in. high; fl. pedunc.; stam. 12-15.

\%. S. pusilla: leaves linear, obtuse and short, a little flattened, foliaceous at the summit; scape simple, mostly shorter than the leaves; flowers monoe cious, few; fertile flowers deflexed.

HAB. Muddy shores. Aug. 4. Scape 2-4 ine high: A. very small, fertile subsolit. 
550. QUERCUS. Oak. Corylacex.

* Fructification biennial; leaves setaciously mucronate.

$\dagger$ Leaves entire.

1. Q. Phellos: leaves deciduous, linear-lanceolate, attenuate at each end, very entire, smooth, mucronate; acorn roundish.

HAB. Sandy woods. A large tree; young leav. dentate; acorn small.

2. Q. imbricaria: leaves deciduous, oblong, acute at each end, mucronate, very entire, shining, pubescent beneath ; cup flat ; scales broad-ovate; acorı subglobose.

HAB. Woods and river banks. A large tree.

$\dagger \dagger$ Leaves dentate, or with short lobes.

3. Q. heterophylla: leaves on long petioles, ovatelanceolate and oblong, entire, or coarsely toothed; cup hemispherical ; acorn subglobose.

HAB. River banks. A hybrid?

4. Q aquatica: leaves obovate-cuneiform, smooth, very entire, obscurely 3 -lobed at the end, with the middle lobe largest; cup hemispherical; acorn subglobose.

HAB. Swamps. Tree middle-sized; leaves very variable.

5. Q. triloba: leaves oblong-cuneiform, acute at the base, somewhat 3-lobed at the end; lobes equal and mucronate, tomentose beneath; cup flat; acorn depressed-globose.

HAB. Fine barrens. Tree small.

6. Q nigra: leaves coriaceous, cuneiform, subcordate at the base, dilated, and retusely 3 -lobed at the summit, when young, mucronate, smooth above, rusty pubescent beneath; cup turhinate, with the scales obtuse and scarious; acorn short-ovate.

HAB. Pine barrens. Tree 10-20 ft. high; acorns abundant.

\%. Q. tinctoria: leaves ovate-oblong, slightly lobed, pubescent beneath; lobes oblong, obtuse, mucronate; cup tlat; acorn depressed-globose. 
HAB. Woods. A large tree; bark rough, blackish.

3. Q. discolor: leaves oblong, pinnatifid-sinuate, pubescent beneath ; lobes oblong, dentate, setaceous. ly mucronate ; cup turbinate, acorn orate.

HAB. Upland forests. A large tree; young leaves doreny on both sides.

\section{$\dagger \dagger \dagger$ Leaves decply sinuate and lobed.}

3. Q. coccinea : leaves long-petiolate, oblong, deeply sinuate, smooth ; lobes divaricate, dentate, acute, setaceously mucronate; cup turbinate, scaly ; acorn short-ovate.

HAB. Woods. A large tree; leav. bright green; cup with prom. scales

10. Q. rubra: leaves long-petiolate, smooth, obtusely sinuate; lobes rather acute, dentate, setaceously mucronate; cup flat, nearly smooth; acorn subovate.

HAB. Woods. A large tree; leav. bright green; sinuses large, rounded.

11. Q. Catesbaei: leaves on short petioles, cuneate at the base, oblong, deeply sinuate, smooth; lobes $3-5$, divaricate, dentate, acute, setaceously $\mathrm{mu}^{*}$ cronate; cup turbinate, broad; scales obtuse, those of the margin inflexed; acorn subglobose.

HAB. Pine barrens. 'Shrubby; 6-15 ft. high; leav. subsessile.

12. Q. falcata: leaves on long petioles, obtuse at the base, woolly beneath, 3-lobed and sinuate; lobes falcate, setaceously mucronate, terminal one longer; cup crateriform; acorn globose.

HAB. Dry woods. A large tree; leav. with 3-5 large lobes, shining above.

13. Q. palustris: leaves on long petioles, oblong, deeply sinuate, smooth; axils of the veins villous beneath; lobes divaricate, dentate, acute, setaceously mucronate; cup flat, smooth; acoru subglobose.

IIAB. Wet woods. A large tree; acorns small, abundant.

14. Q. Bannisteri: leaves on long petioles, obovate, cuneiform, 3-5 lobed, entire on the margin, grayish-tomentose beneath; lobes setaceously mucronate ; cup subturbinate; acorn subglobose. 
HAB. Sandy fields and mountains. Shrub $4-6 f t$. high; acorns abundant.

* Fructification annual; fruit pedunculate; leaves arenless.

\section{$\dagger$ Leaves sinuate.}

15. Q. obtusiloba: leaves oblong, sinuate, cuneate at the base, pubescent beneath; lobes obtuse, the upper dilated ; cup bemispherical ; acorn oval.

HAB. Woods. A middling large tree; leaves mostly 5-lobed; cup deep.

16. Q. macrocarpa: leaves woolly beneath, deeply lyrato-sinuately lobed ; lobes obtuse, repand, upper ones dilated, cup deep, with the upper scales bristly ; acorn short-ovate.

HAB. Woods. A large tree; branches scabrous; acorns very large.

17. Q. olivaformis: leaves oblong, smooth, glaucous beneath, deeply and unequally sinuate-pinnatifid; fruit elliptical-ovate; cup very deep, crenate above; acorn elliptical-oval.

$\mathrm{HAB}$. Woods. A large tree.

18. Q. alba: leaves oblong, pinnatifid-sinuate, pale or pubescent beneath ; lobes oblong, obtuse, mostly entire ; cup deep, tuberculate; acorn ovate.

IIAB. Woods. A very large tree; bark rehite; leav. pubes. beneath when young.

\section{$\dagger \dagger$ Leaves entire, dentate.}

19. Q. Prinus: leaves on long petioles, obovate, acute, pubescent beneath, coarsely toothed; teeth unequal, dilated, callous at the tip ; cup deep, attenuate at the base; acorn ovate.

HAB. Shady woods. A large tree.

20. Q. bicolor: leaves on short petioles, oblong-obovate, whitish-tomentose beneath, coarsely toothed, entire at the base, teeth unequal, dilated, rather acute, callous at the tip; fruit in pairs on long peduncles; cup hemispherical; acorn ublong-orate.

HAB. Woods. A large tree; pedunc. long and slender; acorn pubes.

ß. mollis : leaves merely toothed, not pinnatifid; under side partly ferruginous and softly pubescent. 
HAB. Swampy elevated forests. Tres 60-70 $\mathrm{ft}$. high.

21. Q. montana: leaves on petioles of middling length, broad-obovate, oblong, white-tomentose beneath, shining above, coarsely toothed, obtuse and unequal at the base; teeth subequal, very obtuse; fruit in pairs, on short peduncles ; cup hemispherical; scales tuberculate and rugose ; acorn ovate.

IIAB. Rocky woods and mountain sides. A large tree; fr. middle sized.

22. Q. castanea: leaves on long peduncles, oblonglanceolate, obtuse at the base, acuminate, tomentose beneath, coarsely toothed; teeth subequal, dilated, obtuse ; cup hemispherical ; acorn ovatesubglobose.

HAB. Rocky and mountain woods. A large tree; fr. on short pedunc.

23. Q. Chinquapin: leaves on short petioles, obovate, acute at the base, coarsely toothed, glaucous beneath ; teeth subequal, dilated, callous at the tip ; cup hemispherical ; acorn ovate.

HAB. Barren woods. A shrub 3-4 ft. high; acorns small, numerous.

\section{CORYLUS. Hazel-nut. Corylacece.}

1. C. americana: leaves roundish, cordate, acuminate; involucrum roundish-campanulate, larger than the subglobose nut, border dilated, coarsely serrate.

HAB. Fields and woods. April. A shrub $3-5 \mathrm{ft}$. high; nuts large, edille.

2. C. rostrata: leaves oblong-ovate, acuminate; stipules linear-lanceolate; involucrum campanulatetubular, longer than the uut, two.parted, with dentate segments.

HAB. Rocky woods. May. A slirub 2-3ft. high; involuc. with a long rostrum.

552. FAGUS. Beech. Corylacere.

F. ferruginea: leaves ovate-oblong, acuminate, pubescent beneath, ciliate on the margin, coarsely toothed, obtuse at the base and unequally subcordate; nut ovate, acutely triangular, very acute. 
HAB. Woods. A large tree; bark smooth; fruit muricate; leav. ribbed.

?. F. sylvatica; leaves ovate, acuminate, slightly dentate, ciliate on the margin, acute at the base; nut ovate, triquetrous, obtuse, but mucronate.

HAB. Woods. A large tree with num. branch.; leav. bright green.

\section{CaStanea. Chesnut. Corylacere.}

1. C. vesca, f. americana: leaves lanceolate, acuminate, mucronately serrate, smooth on both sides.

HAB. Woods. June. A large tree; trunk straight; leav. large; sterile spik. filif.

2. C. pumila: leaves oblong, acute, mucronately serrate, tomentose and hoary beneath.

HAB. Sandy woods. A shrub or small tree; fruit small, very szeet.

\section{BETULA. Birch. Salicince.}

1. B. populifolia: leaves deltoid, lung-acuminate, unequally serrate, very smooth; petioles smonth; strobile pedunculate; scales with roundish lateral lobes.

HAB. Rocky woods and mountiins. May. A small or middle sized tree.

2. B. excelsa: leaves ovate, acute, serrate; petioles pubescent, shorter than the peduncle; strobile ovite, erect; scales with rounded lateral lobes.

HAB. Woods. A large tree; bark smocth and yellowish, somewhat frasrunt.

3. B. nigra: leaves rhombic-ovate, doubly serrate, acute, pubescent bereath, entire at the base ; strobile ovate ; scales villous, with the segments linear and equal.

HAB. Banks of rivers. A middle sized tree; leav. acutely serrate.

4. B. papyracea: leaves ovate, acuminate, doubly serrate, the veins beneath hairy; perluncles smooth; strobile nodding, pedunculate; scales with the lateral lobes short and suborbicular.

HAB. Woods. A large tree; cuticle tough, com. posed of numerous membranaceous layers.

$\mathrm{H} \mathrm{h}$ 
5. B. lenta: leaves corlate-ovate, sharply serrate. acuminate; nerves bereath and the petiole hairy: strobile erect ; scales smooth, the lobes equal and obtuse, with elevaterl veins.

HAB. Wonds. A large tree; bark aromatic; brunchlets dark, spotted.

8. B. pumila: young brinches pubescent, not punctate; leaves orbicular-ohovate, petiolate, tbickly pubescent benenth ; simbile cylindrical.

HAB. Mountain bogs. June. Shrub 2-3 ft. high; leav, 1 in. long, on short pet.

7. B. glandulosa: branches glandularly punctate, smooth; leaves ohovate. serrate, entire at the ba-e, smooth, subscsile; strobile oblong; scales half 3-cleft; seed orbicular, with a narrow margin.

HABB. Mountain swampls. Shrub $2 \mathrm{ft}$. high.

3. B. nana: very smooth leaves; orbicular, crenate, reticulate beneath; scales of the strobile decply 3-parted; segments oblong ; sced orbicular, nearly wingless.

HAB. High mountains. Shrut $1-2$ ft. high; ?cau. $\frac{1}{3}$ in. long.

\section{CARPINUS. Hornbeam. Coryiacce.}

C. anericana: leaves oblong-rvate, acuminate, unequally serrate; strobile with 3 -parted scales, the middle segment ohlique, toothed on one side.

HAB. Woods. May. A small tree; fertile aments loose; scales foliaceous.

556. OSTRYA. Hop hornbeam. Corylacece.

O. virginica: leaves ovate-oblong, cordate at the base, acuminate. unequally serrite; strobile oblongorate, erect ; buids acute.

IIAB. Woods. A small trce; cones large, resembling hops.

557. PLATANUS. Buttonwood.

P. occidentalis: leaves lobed-argular; branches whitish. HAB. Woods Averylarge trec; leav.verybroad; arients glab., pendulous. 
558. LIQUIDAMB.AR. Gum-tree. Corylacec.

L. slyraciflua: leaves palmately lobed; lobes acuminate, serrate, with the sinuses at the base of veins villous.

IIA B. Woods. A large tree ; leuv. 5-lubed, cordare, dark green.

559. JUGLANS. Waliut. Cory!aceæ.

1. J. nigra : Ioflets numerous, ovate-lanceolate, serrate, slightly cordate, tipering to the summit; under surface and petiol s subpubescent; fruit glohose, scahrons, punct ite.

HAB. Woods. A larse tree; leisv. pinnute; leafl. 15-21; fr. lorge, dible.

2. J. cinerea: leafletr tumerous, lancenlate, serrate, rounicrl at the hise. oft pubesc nt berseath ; petioles villous; fruit oblong-ovate, with a terminal projection, viscid and hairy; nut oblon: acumin tte, deeply and irregularly sculptured.

HAB. Woods. A lavge tree; leafl. 15-17; fruit jery viscid; nut eivible.

\section{CARYA. Hickory. Coryliscece.}

1. C. ementosa: Iraliets ahout 7 , oblong-larceolate, acuminate, slishtly serrate, pubescent and scabrous beneath, the oild one sessle; ament tiliform, tomentose ; firut roundish, snooth ; pericarp very thirk; wut somewhat 6 -angled; shell thick and very hard

HAB Woorls. I larye tree; leav. pinnate; veins pubes. above; nu:s alible.

2. C. albit: leaves 5-7, on long petiolrs, sblong-lanceoliate, acuminite. sharply serrite villows heneath, the ord one secille; atm nts filiorm, smooth; fruit drepressed-globose; nut com. pressed.

HA B. Wools. A large tree with scaly bark; fruit lurgere; nut rehite thin-shel!ed.

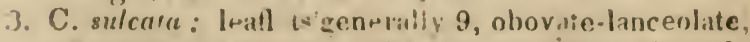
acumnate, serrate, pubescent bericalls, the odr 
one subsessile and attenuate at the base; fruit roundish, 4-angled; nut subglobose, slightly com. pressed, conspicuously mucronate.

HÄB. Mountain woods. A large tree; nuts large, with very thick pericarps.

4. C. amara: leaflets generally 9 , ovate-oblong, aruminate, acutely serrate, smonth on both sides: fruit subglobosé; nut smooth, mucronate, with the shell frigile.

HAB. Dry wods. A large tree; nerves of the leav. pubes. beneaih; nuts bitter.

5. C. porcina : leaflets 7 , orate-lanrenlate, acuminate, serrate, smooth on both sirles; fruit oblong, or obcordate; nut snooth, very hard.

IIAB. IVoods. A. large tree; leafl. $5-7$; nuts. small, very bitter.

\section{ARUM. Wake-robin. Aroidece.}

1. A. dracontium : stemless ; leaves pudate ; leaflets lanceolate-oblong, entire; sparlix =ubulate-fliform, longer than the oblong convolute spathe.

HAB. River banks. June-July. 4. Petiole 12-18 in. long; leav. large; spath. șhort.

2. A. triphyllum: stemless; leaves ternate; leaflets ovate, acuminate, very entire; spadix clavate; spathe ovate, acuminate, convolute below, that and bent above.

HAB. Wet shady places. May-June. 4. Root tub. acrid; spath. green or purp.; berry red.

562. LECONT1..* Aroidea.

1. virginica:

HAB Swamps. July. 4. Leav. petiol., oblong, hastate-cordate, with the Inbes obtuse; spathe lanceolate, invointe; border undul.; spad. slender.

563. CALLA. Water arum. Aroidec.

C. puinstris: leaves cordate; spathe flat; spadix covered with perfect flowers.

* A new genus, separated from Arum by W. Cooper, Esq. 
HAB. In water. July. 4. Ront thick, crceping; leav. acum.; spath. oval, whice iaside.

\section{MONADELPHIA.}

564 PINUS. Pinc. Coniferce.

* Leuves solitary, distinct at the base. aBres.

1. P. balsameat lear: s solitary flit, emarginate or entire, glaucous benesth, sumewhat pectinate, recurved-spreading, sub-erect above; cones cylin dric, erect; hracts abhreviate, obovate, conspicurusly mucronate, subseriniate.

HAB. Mountains. A small tree; leav. 3-10 lin. long, dense; scules of the cones thin.

3. Fraseri: leaves short, emarginate, subsecund, erect alove; cones ovate-oblong; bracts elongated, incisely denticulate.

HAB. High mountains. Smaller than the preced.; leaves shorter; cones small.

2. P. canadensis: leıves :olitary, flat, denticulate, nearly in two rows : cones ovate, terminal, scarcely longer than the leaves.

HAB. Rocky woods and mountains. A large tree; branch. and leav. horizont.; leav. lin., obtuse.

3. P. nigra: leaves solitary, quadrangular, erect, straight; cones ovate; s^ales. elliptical, undulate on the margin, erect, summit denticulate.

$\mathrm{H} \Lambda \mathrm{B}$. Mountain swamps. A middle sized tree; leav. dense, durk green; cones 1--2 in long.

4. P. rubra: leaves solitary, subulate; cones oblong, obtuse; scales rounded, somewhat 2-lobed, entire on the margin.

HAB. Maine.

5. P. alba: leaves solitary, quadrangular, incurved; cones subcylindrical, loose; scales obovate, very entire.

HAB. Mountain swamps. A smull tree; leav. pale green, not crozeded.

* Leaves 2-5, in a short cylindrical sheath. PINUG.

6. P. inops: leaves by pairs, short ; cones recurved, H h 2 
oblong-conical, as long as the leaves; spines of the scales subulate, straight.

HAB. Pine barrens. A middle sized tree; leav. 1-2 in. long; concs 2 in. long.

7. P. resinosa: leaves elongate, by pairs, with long sheaths; cones ovate-conical, rounded at the base, mostly solitary, half as long as the leaves; scales dilated, unarmed.

HAB. Dry wonds: Alarge tree; bark red, smooth; cones large; scales not mucronate.

3. P. Banksiana: leaves short, by pairs, rigid, divaricate, oblique ; cones recurved and twisted; scales unarmed.

HAB. Barren rocky places. A small straggling tree; leav. 1-2 in. long.

3. P. variabilis: leaves slender, geminate and by threes, canaliculate; cones mostly solitary, ovateconical ; scales with a subulate incurved point.

HAB. Woods. A large tree; leav. mostly by pairs, 4-5 in. long; cones small.

10. P. rigida: leares by threes, with short sheaths; sterile aments erect-incumbent: cones ovate, scattered or clustered; spines of the scales reflexed.

HAB. Barren sandy soils. A large tree; bark thick and rough; cones large.

11. P. scrotina: leaves by threes, elongate; sterile aments erect, incumbent ; cones ovate; spines of the scales straight, very slender.

HAB. Swamps. A large tree resembling the preceding.

12. P. Strobus: leares by fives, slender; sheaths very short; cones pendulous, cylindrical, longer than the leaves; scales loose.

HAB. Fertile soil. A very large tree; leav. very delicate; cones 3 in. long.

* * Lcares fasciculate, (deciduous.) I.ARIx.

63. P. microcarpa: cones roundish, few-flowered; scales inflexed; bracts elliptic, obtusely acuminate.

HAB. Swamps and low grounds. A pretty large tree; scales red, brown, or white. 


\section{THUYA. Arbor vitæ. Coniferce.}

T. occidentalis: branches ancipitous; leaves imbricate in 4 rows, ovate-rhomboidal, appressed, naked, tuberculate ; cones obovate ; interior scales truncate, gibbous below the summit.

HAB. Rocks and mountains. A small tree; branch. irreg., tough; leav. perennial.

\section{CUPRESSUS. Cypress. Coniferce.}

1. C. thuyoides: branchlets ancipitous; leaves imbricale in 4 rows, ovate, tuberculate at base; cones subspherical, angular.

HAB. Swamps. A large tree; leav. minute, peren.; cones very small.

2. C. disticha: leaves distichous, flat; sterile florets paniculate, leafess; cones spherical.

HAB. Swamps. A very large tree; leav. small, lin., acute.

567. ACALYPHA. Three-seeded mercury. Euphorbiacece.

A. virginica: pubescent; leaves on short petioles, lanceolate-ıblong, serrate; involucrum cordate, ovate, dentate, axillary, nerved, denate.

HAB. Diy gravelly soil. Ang. ๑. Stem 815 in. high, erect, pubes.; invol. subsess.

563. PHYLLANTHUS. Euphorbiacece.

P. obovatus: leaves alternate, oval, obtuse, smooth, somewhat distichous; flowers few, axillary, pedicellate, nodding ; stem erect ; branches distichous. HAB. Gravelly banks. June-July. $\odot$. Stern 12 in. high; leav. entire; stam. 6.

369. MELOTHRId. Small creeping cucumber. Cucurbitacece.

11. pendula : leaves somewhat reniform, lobed and angled, slightly hispid; berry oval, smooth.

HAB. Shady hanks. June. ๑. Slender, climb. ing; stem hairy; ll $_{\text {. yell. }}$ 
570. MOMORDICA. Balsam-apple. Cucurbitaceu.

M ? echinuta: perirarp a pomacenus berry, 4-secded, inflited, roundish, sstise-echinate, leaves cordate, angularly 5-lobed, acuminate, entire; calyx 6-cleft; corolla 6-parted.

HAB. Banks of rivers. Ang. $\odot$. Climbing; $f$. small; fr. $1 \frac{1}{2} \mathrm{in}$.long.

\section{SICrOS. Single-seeded. Cucurbitacea.}

S. angulata: leaves cordate, 5-angled, dentate, scabrous ; fruit clustereci, hispici.

HAB. Bank of rivers, \&r. ¿. Climbing; stem pubes.; leav. petiol.; fr. small, ovatc.

\section{. DIOHCI.}

\section{DIINDKIA.}

57\%. VALLISNERIA. Stam. FL. Spathovale, 2-parted. Spadix covered "ith minute flowers. Cal. 3-parted. pisticlif. Spalh hifid, 1-flowered. Cal. 3-parted, superior. Corr. 3-petalled. Stig. ligulate. bifit. Caps. valveless, 1-celled. Sceds numerous, attached to the sirles.

573. SALIX. STAM. FL. Ament cylindric; scales 1 flowered, imbricate, with a nectariferous gland. at the bise. Cal. and Cor. 0. Slam. 1-5. FERTILE FL. Scales of the ament 1-flowered. Cal. and Cor. 0. Stig. 2, nosily bifirl. Caps. 1-celled, 2-valved, mary-seeded. Seeds comose.

574. FR tXINUS. Cul. 0, or 3-4-cleft. Cor. 0, or 4-petalled. Stam. \&. Capis. 2 celled, 2-seeded, compressed and foliarenus it the extremity. Seed sulitary, penculous.-Polygamous.

\section{TRIANDRIA.}

575. EMPETRU M. Cal. 3 parted, persistent. STAM. EL. Pet. 3, marcescent. Stam. 3 ; filaments 
long; anth. 2-parted. FERTILE. Germ. superior, depressed; style 0 , or very short ; stig. 9, reflexed, spreading. Berry round, 1-celled, 2-6-seeded. Seed erect, bony.

\section{TETRANDRIA.}

576. MYRICA. Ament ovate-oblong; scales lunulate. STAM. FL. Stain. 4-6; anth. 4-valved. FERtile. Germen 1; stig. 2. Drupe 1 celled, 1 serded

577. VISCUM. Cal. with the margin entire or a litthe pronment. Pet. 4, short, united at the base. STAM. FL. Anth. 4, se-sile, adnate with the petils. FERTILE. Germ. crowned with the margin of the calyx ; stig. 1. Berry globose, 1-seeded.

\section{PEN'TANDRIA.}

578. NYSSA. PERFECT Fl. Cal. 5-parted. Cor. 0. Pistil. 1. Drupe inferior. Nut 1 -seederl. Sterile Stam. 5, 8, 10, or 12, inserted around a peltate gland.

579. ZANTHOXYLUM. stam. FL. Cal: 5-parted. Cor. 0 Stam. 3-5-6. Fertile. Caps. 35-celled, each 1-seeded.

580. ACNIDA. stam. fl. Cal, 5-parted. Cor. 0. fertile. Cial. 3-parted. Cor. 0. Styles 0 ; stig 8, sessile. Caps. 1-seederi.

581. HUMULUS. sтам. FL. Cal. 5-leaved. Anth. with 2 pores at the extremity. Cor. 0. FERrile. Cal. 1-leaved. large, persistent, concave, entire. Cor. 0. Styles 2. Seed 1.

\section{HFXANURIA.}

582. SiIILAX. stam. fl. Cal. 6-leaved. Cor. 0. Anth. adnate to the filaments. FERTILE. Style minute stig. 3. Berry 3-celled, superior, 1, 2 , or 3-seeded.

583. DiOSCOREA. 8ter. Fl. Cal. 6-parterl. Cor. 0. rertile. Styles 3. Caps. 3-celled, trian 
gulsr. compressed; cells 2-sepded. Sceds with membranacerus margin:.

384. GLEDI I'SCHIA. PERFECT. Cal. 6-8-parted, decidunus, 3 or 4 of the exterior segmente smiller. Cor. 0. Stam. 5-6, rarely 8. Leguine flutly compressei, 1, or many-seeded. sterile. Cal. subturbinite, 5-8-pirted, 35 of the seginents interior. Sium. 6-8.

\section{OCIANDHIA.}

385. POPULUS. Aments cylinilrical; scales lacers. ted. STERILE FL. Sia:n. ó--30, stited on a turbinate obilque entire calıx. FERTILE. Cal. turbinate. Stig. 4. Caps. upuerior, 2-cefl.r, 2-valver!, masy-seeded. Seeds surrounded with Iong hairs.

586. DIOSHYRUS. Cal. 4-6-cleft. Cor. ureeolate, 4-6 cleft. Sterile Fi. Stam. 8-16; filam. often producing ? awhirs. FERTILE. Sig. 45. Berry 8-12-sperlerl.

987. SHEPHERDIA sterILE FL. Cal. 4-cleft. Cor. 0. Stail. 8, incluleil, alternating with 8 uglatis. Fенти.е. Cal. 4-cleft, campanulate, superior. Style 1; sigg. oblıque. Berry 1-seeded. -

\section{ENNEANDRI.}

388. ELODEA. Spath bitid. STEhile Fl. Cor. 3puilled Siam. 9. 3 of them interior. FERTuLe. Cal. 3-parted; twhe viry lnng. Pet, 3. Sterile tilanents 3. Utriculus about 3-seeded. Seeds cyludric. .

\section{HECANDIII.}

389. GYMNOCL ADUS. Cal. $\{1+m): r, 5$-cleft. Cor. 4 - lofi. Cir 5.prtillid. STERILE FL. Stam. 10. Fertilf. Sig/e 1. l.egume 1-celleri, intwindly somew hat pulpy. Seeds roundish, latge, hart. 


\section{POLYANDRIA.}

390. MENISPERMUM. STERILE Fl. Cal. 2-bracted, about 6-leaved, caducous. Pet. 6-9, glandular, minute, and retuse. Sitam. 16-24; anth. adnate to the filaments, 4 -Inbed, 2-celled. FERTILE. Germens and stylfs 3-6. Drupes mosily solitary, 1-seeded. Niut lanate, compressed.

\section{MONADE: PIIA.}

j91. JUNIPERUS. STFRILE FL. Ament orate; scales verticillate, veltate. Arth. 4-8, 1-celled. FERTILE. Ament sloboss ; scules 3, cortlunate. Stig. gatping. Berry with 3 hony 1 -ceeded uuts. surrounderi with the united and le-hy scales.

992. TAXUS. Floreers surround ind ivith numerous scales. Sterite fL. Stan. 3--10; anthers peltate. Fentile. Siyle 0; stig. concave. Drupe deshy, open at the extremity. Nut 1seeded.

\section{IHOECIA.}

\section{DIANDRIA.}

572. VALLISNERIA. IIydracharilua.

Y. spiralis, $\beta$. anericana : leaves linear and obtuse, equal the whole length, 3-norved; margiu aculeately serrulate; sterile pectuncles very short, fertile, lons spiral.

HAB. Still or trr. Aug H. Lcay. S-S lin. l.road; caprs cylind., loug ; pet. filiform.

573. SALIX. Wiliow. Salicina.

* Lscazcs entire or obsoletcly scrrate.

1. S. candida : leaves linear-lancenlate, very long, obscurely denticulite at the extreonity, pubescent ahore, white and tomentose beneath: margin re- 
volute; stipules lanceolate, as long as the petioles; aments cylindric ; scales obnvate-lanceolate, with a very long villus.

HAB. Woods. April. h. Shrub 3-1 ft. high.

2. S. viminalis: leaves linear-lanceolate, obscurely crenate, white and silky beneath; stipules very small, sublanceolate; branches it raight; germens subsessile, lanceolate; style filiform; stigma linear, mostly entire.

HAB. River banks, \&c. April. A pretty large tree; fil. yell.; anth. orange. $\$$.

3. S. Muhlenberyiana: leaves lanreolite, rather acute, nearly entire, whitish, pubescerit. rugose and veiny beneath, revolute on the m.ruin; stipules deciduous, lanceolate; scales oblong, villous on the margin; germen ovate-lanceolate, silky-villous, on a conspicuous perlicel; style short; stigma bifid.

HAB. Dry woods. April. . Shrub 3-5 ft. high; branch. gr.-yell.; anth. purp. und yell.

4. S. tristis: leaves linear-lanceolate, acute at each end, revolute, on the margin, smoothish above, beneath rugosely veiny and tomentose; stipules 0 .

HAB. Dry woods. Shrub 3-4 fi. high.

5. S. recurvata : leaves obovate-lanceulite, acute, very entire, smooth, glaucous bereath, silky when young; stipules 0 ; aments rerurved; scales black at the tip, with hairs as lo:, as the ovate, silky, and subpedicellate germen; style very short; stigmas bifid.

HAB. Shady woods. Shrub 2-3 ft. high; Uranch. brozen, smooth; buds yell.

6. S. pedicellaris: branches smonth; leaves obovatelanceolite, acute, very entıre, smonth, and of the same colour on both sides; stipules 0 ; aments pedunculate; scales oblong, scarcely hisiry, half as long as the pedicel; gertnen ovate-oblong, on a very long pedicel; stigmas sessile, bifid.

HAB. Mourtains. April.

7. S. rosmarinifolia: leaves straight, linear-lanceolate, acute at each end, very entire, deciduously pubescent above, silky beneath; scales oblong, ob. 
thse, cilinte; germens pedicellate, lanceolite, vil lous ; stigmas subsessile, bifid.

HAB. adows and mountain swamps. Shrub 3 ft. high; branch.pubes.; leav. $1 \frac{1}{3} \mathrm{in}$. long.

a. S. repens: stem procumbent ; leaves acnte, nearly entire, somewhat downy, glaucous, mostly very silky beneath; germens on a long pedicel, lanceolate, very silky ; styles short ; stigmas bitil.

IIAB. High mountains. Shrub sinall, usually procumb. - V ariable.

* Leares remotely and obtusely serrate.

3. S. conifera: leaves oblong-lanceolate, remotely scr rate, acute, smooth above, tomentose beneath; stipules lunate, subdentate; scales lanceulate, obtuse, villous; germens pedicellate, lanceolute, silky ; style bifid ; stigrnas 2-loberl.

II.AB: Low thickets. April. Shrub $4-3 j$ t. high; frequently with excresences on the branches.

10. S. m:yricoides : leaves oblong-lanceolite, acute, ob. tnsely serrate, smooth, shlucous bene:uth ; stipules ovate, icute, serrate; aments villous, lealy at the base; scales lanceolate, villous, (black ;) germens on long pedicels, lanceolate, smooth; style bitid; stigmas bifil.

HIAB. Woods. A sinall shrub; branch. green; young ones purp., smooth.

11. S. prinoides: leaves ovil-ohlong, :kute, remotely undulate-serrate, smooth, glaucous beneath; stipules semicordate, incisely dentite; germena pediccllate, ovate, acuminite, silky ; style long ; stigmas bitisl.

HIB. Wet thickets, April. A shrub $6-8 j$ ft. high ; ainents early, villous.

1 s. S. discolur : leaves oblong, rather obtuse, smooth, remotely serrate, glaucous beneath; stipules deciluous, lancenlate, serrate; scales oblong, ncute. (black, hairy ; germens subsessile, lanceolate, tomentose, style of middling length ; stigmas?-prarted.

HAB. Low grounds. April. A small erce; brancí. dirk brown; aments rather late.

13. S. angustuta: leaves lancenlate, acute, very long. gradually narrowed toivirils the base, serrulate. I i 
very smooth, colour nearly uniform; stipules semicordate; germens pedicellate, ovate, smooth; style bifid; stigmas 2-lobed.

IIAB. Banks of rivers. Leaves very long. Re sembles No. 11.

\section{** Leaves closely and acutely serrate.}

14. S. nigra: leaves lanceolate, acute at each end, serrulate, green on both sides, smooth except the petiole and the midrib above; stipules dentate; aments cylindric; scales oblong, very villous; filaments $3-6$, bearded at the base ; germens pedicellate, ovate, smooth; style very short; stigmas bifid.

HAB. Banks of rivers. May. A small tree; branch. smooth; first leaves pubes.; aments long.

15. S. lucida: leaves ovate-lanceolate, long-acuminate, rounded at the base, serrate, smooth on both sides. shining; stipules oolong, serrate; aments triandrous; scales lauceolate, obtuse, hairy at the base, serrate and smooth at the tip; germens lanceolatesubulate, smooth; style bilid; stigmas obtuse.

IIAB. Swamps. May. A small tree; leav thick; braneh. yell.-br.; aments late.

16. S. rigida: leaves oblong-lanceolate, acuminate, subcordate at the base, rigid, smooth, acutely serrate, the lowest serratures long; petiole villous; stipules large, corlate, obtuse ; stamens 3; scales lanceolate, (black,) woolly; germens on long pedicels, smooth; style very short; stigmas 2. parted.

HAB. Low thickets. Iray. A small tree; branch. green, tough; aments late.

17. S. cordata: leaves oblong-lanceolate, acuminate, cordate at the base, acutely serrate, smooth; stipules large, ovate-roundish, serrate; stamens 3 ; scales lanceolate, (black;) germens pedicellate, lanceolite, smooth; style very short; stigmas bifid.

HAB. Swamps. April-May. A shrub 6-3 fto high; leav. large, 1 in. broad.

B. falcata: leares narrow-lanceolate, very long, sulkfalcate. 
IIAB. Swamps. Leav. 6-8 in. long.

18. S. grisea : leaves lanceolate, acuminate, serrulate, smooth above, silky or naked beneath; stipule: linear, deflexed, deciduous; scales oblong, hairy, black at the tip; germens oblong, pedicellate, silky ; stigmas sessile, obtuse.

IAL. Low bushy places. April. Shrub 6-8 ft. high; branch. purp., brittle at base.

19. S. vitellina: leaves lanceolate, acuminate, with thickened serratures, smooth above, paler and somewhat silky beneath ; stipules 0 ; aments $\mathrm{cy}$ lindrical, scales ovate-lanceolate, pubescent outside; germens sessile, ovate-lanceolate; stigmas subsessile, 2-lobed.

IAB. Read sides, \&c. May. A pretty large tree; burk and trigs yell. \$.

574. FRAXINUS. Ash. Oleinece.

1. F. sambucifolia: leaflets sessile, ovate-lanceolate, serrate, rugose and shining; base rounded and unequal; axils of the veins villous beneath; flower's naked.

IIAB. River banks and woods. Aprit. A large tree; buds deen blue; young shoots dotted, green.

2. F. acuminata: leatlets petiolate, oblong, shining, acuminate, very entire, or slightly toothed, glaucous beneath; flowers calyculate.

HAB. Woods. May. A large tree; first leav. downy; suinmer leitv. gr. above, wh. beneath.

3. F. pubescens: leatlets petiolate, elliptical-ovate, serrite; under surface, petioles, and young branche: tomentose; flowers calyculate.

11AB. Wnorls. Mily. A large tree; leav. 12-15 in. long; leafl. often nearly entire, 3-4 pairs.

4. F. juglandifoliu: leafles petiolate, ovate, opake, serrate. glaurou ${ }^{\circ}$ beneath, axils of the veins pubescent; branches amooth; Howers calyculate.

II А B. Banks of rivers. I sinall tree; lear. 6-15 iu. long; seeds smaller than No. 2. 


\section{'IRIANDRIA.}

575. KMHETIUM. Crow-berry. Empetrcu.

l. nigrum : procumbent; leaves linear-oblong.

HAB. High mountains. June. Shrub small, evcr srecen; leav. densely imbricate; $f$. axill.

576. MYRICA. Gale. Salicince.

1. MI. Gale: leaves lanceolate, broader above, ser. rate; sterile ament imbricated; scales acuminate, ciliate; fruit sca!y-capitate.

HAB. Borders of ponds and about mountain lakes. May. Shrub 3-4 ft. high; fruit spicy.

2. M. ccrifera: leaves cuncate-lanceolate, with a few serratures at top, acute; sterile aments loose; scales acute ; fruit spherical, naked, distinct.

IIAB. Dry woods and copses. May. Shrub 2-6 ft. high; fruit small, covered with wax.

577. VISCUM. Misseltoe. Loranthex.

1. zericillatum: leaves cuneate-oval, 3-nerved, obtuse; spikes axillary, solitary, about as long as the leaves; sterile flowers mostly trifid.

HAB. Parasitic on trees. Branch. oppos.; sterile A. mostly trifid; berry itite, diaph.

\section{PENTANDRIA.}

\section{NYSSA. Tupelo. Combretacer.}

1. N. rillosa: leaves oblong, very entire, acute at each end, the petiole. midrib, and margin villous; fertile peduncles $3-6$-flowered; nut short-oborate, obtusely striate.

IIAB. Low woods. June. A large tree; branch. horizont.; fl. sinall, gr.; drupe dark blue.

2. N. biflora: leaves ovate-oblong, very entire, acute at each end, smooth; fertile peduncles 2-flowered ; drupe short-obovate; nut obtusely striate.

HAB. Swamps. June. A large trce: drupe dee? blue. 
57. ZANTHOXYLUM. Tooth-ach tree. Riulacece.

Y. fraxineum: prickly; leaves pinnate; leaflets orallanceolate, subentire, sessile, equal at the base; petioles unarmed; umbels axillary.

IIAB. Rocky woods. April. Shrub 3-15ft.high; prickles strong; leafl. $4-5$ pairs.

580. $\Lambda$ CNIDA. Wild-hemp. Chenopodece.

1. A. cannabina: leaves ovate-lanceolate; capsules smonth, acutely ingled.

HAls. Salt marshes. July-Aug. ๑. Stem 2$4 f t$. high, sinooth; leav. alt.; fl. small, gr.

2. A. rusocarpa: leaves oval-lanceolate; capsules obtusely angled, rugose.

IIAB. Fresh-water marshes. July. ๑ +

\section{HUMULUS. Hop. Urticer.}

H. Lupulus.

IIAB. Hedges, \&c. Aug. 4. Stem trein., sctbrous; leav. oppos., 3-5-lob., rough; $f$. gr.

\section{IEXANDRIA.}

582. SHIIIAX. Ruugh bind-weed. Asparager.

* Stern frutescent.

1. S. Sarsaparilla: stem prirkly, slightly 4-angled; leaves unarmed, ovate-lanceolate, cuspidate, somewhat 5-nerved, glaurous beneath; peduncles longer than the petioles.

IIAB. Low thickets. July. Sten climbing; prickles scuttered; ber. black, 3-seeded.

2. S. rotundifolia: stem prickly, subterete; leaves unarmed, roundislı-ovate, short-icuminate; cordate, 5-7-nerved; berries spherical.

IIAB. IIedges, \&ic. June. Stem climb.; branch. quadrang.; ber. black; $f$. umbel.

$\therefore$ S. cadisca: prickly ; leaves ovate, mucronate, membranaceous, 5-nerved; common peduncles gcascely longer than the petioles: 
HAB. Fields and dry woods. June. Climiling: stem subang.; petiol. 5-6 lin. long.

1. S. Pseudo-china: stem unarmed; leaves unarmed, those of the stem cordate, of the branches ovateoblong, 5-nerved; peduncles very long.

HAB. Pine barrens and sandy fields. June. Rools large, tuber.; stem climb.

5. S. laurifolia: prickly; branches unarmed; leaves coriaceous, elliptical or elliptical-lanceolate, obtuse, slightly acuminate, shining, 3.nerved; umbels on short peduncles.

HAB. Pine barrens. July. Stem with few prickles; leav. very thick, smooth.

5. 5. pandurata: prickly; leaves ovate-panduriform, acuminate, 3-nerved; common peduncle twice as long as the petiole.

HAB. Sandy woods. July. Leav. smooth and shining. A var. of the preceding?

* Stems herbaceous, unarmed.

7. S. herbacea : stem slightly angled, erect; leaves 5-7-nerved, acuminate, pubesceit heneath, the lower ones alternate, upper verticillate and crowded; peduncles very long, compressed.

HAB. Moist shady places. June. Stem 2-3 $\mathrm{ft}$. high, nearly simp.; ber. black.

3. S. peduncularis: stem terete, climbing; learcs ovate, nearly round, cordate, acuminate, 9-nerved; umbels on very long perluncles.

HAB. Low grounds. June. Stem climb. by tendrils, 5-8 ft. high; lear. smooth; $f$. fetid.

\section{DIOSCOREA. Yam. Asparagece.}

1. D. villosa: leaves alternate, opposite and rerticillate, cordate, acuminate, jubescent beneath, 9 . nerved, the lateral nerres simple.

HAB. Hedges and woods. June. H. Climbing; ster. $f$. in axill. panic., small.

2. D. quaternata : leaves verticillate by fours, and alternate, cordate, acuminate, smooth on both sicles: 7 -nerved; the lateral nerves divided.

IAB. Dry soils. June. 4. Climbing; leav. longer acum. than the preceding. 
584. GLEDITSCHIA. Honey-locust. Leguminosce.

G. triacanthos: branches spiny; spines thick, triple and compound; leaflets oval and oblong; legumes many-seeded.

HAB. A bout plantations. June. A middle-sized tree; spines very long; legumes falcute, long.

3. inermis : branches unarmed.

HAB. With the preceding.

\section{OCTANDRIA.}

585. POPULUS. Poplar. Salicince.

1. P. candicans: leaves cordate, ovate, acuminate, ob. tusely and unequally serrate, white beneath, somewhat triply nerved, reticulately reined; petiole hairy; buds resinous; branches round.

HAl3. Woods. April. A small trce; leav.large: bud's odorous.

2. P. tremuloides: lcaves roundish, abruptly acuminate, dentate-serrate, pubescent on the margin.

HAB. Woods. April. A middle-sized trce; barti smooth; leav. thin, ruther small.

3. P. hudsonica: leaves rbomboil, with a very long acumination, dentate-serrute, smooth; young branches hairy.

11AB. Banks of rivers. Aprit. I large tree branch. spreading; leav. 3-4 in. long.

1. P. grandidentata: leaves rounclish-ovate, acute, with large unequal sinuate teeth; younger ones villous. IIAB. Woods. April. A middle-sizcd trce; youns leav. downy.

B. pendula: branches pendulous.

IIAB. Mountains. Kare.

7. P. ictcrophylla: leaves roundish-ovate, with the sinus small, cordate, sulamuculate, uncinatcls toothed; young ones tomentose.

586. DIOSPYROS. Persimmon. Eibenacce.

D. virgiviana: leaves ovate-oblong, acuminate, smooth. reticulately reined; petioles pubcscent; budsmooth.

HAB. Woods. June. A small tree; $f$. palc orange: fr. rosudish, yell, astring. 
587. SHEPHERDIA. Eleagni.

S. canadensis: leaves oblong-ovate, nearly smooth, above. stellately hairy and scaly beneath; the scales ferruginous and deciduous.

HAB. Borders of lakes and river banks. Aug. I shrub 6-8 ft. high; fl. clust., minute.

\section{ENNEANDRIA.}

583. ELODEA. Haloragea.

L. canadensis: leaves verticillate by threes and fours, lanceolate, oblong or linear, serrulate; tube of the calyx filiform.

HAB. Creeks and still waters. Aug. 4. Stem subm., dichot.; $f$. minute, white.

\section{DECANDRIA.}

589. GYMNOCLADUS. Coffee-bean tree. Leguminosce.

G. canadensis.

IHAB. Woods. May-June. A middle-sized tree:leav. very large, oipinnate; $A$.racem.

\section{POLYANDRIA.}

j90. MENISPERMUM. Moon-seed. Menispermacec.

I. MI: canallense: leaves peltate, cordate, rounded angular; racemes compound; petals 8 .

IIAP. Woods and hedges. July. 4. Climbing: netiol. long; racem. axill. ; $\mu$. small, yell.

\section{MONADELPHIA.}

591. JUNIPERUS. Juniper. Coniferce.

1. J. communis: leaves ternate, spreading, murronate, longer than the berry.

3. depressa: stems prostrate. 
HAB. Dry woods. April-May. A low spreading shrub; leav. shining above, glauc. beneath.

2. J. virginiana: upper leaves imbricate in four rows, ovate-lanceolate, pungently acute, appressed; young ones acerose expanding; trunk arboreous.

HẢB. Dry rocky places. April. A small tree; leav. sometimes ternate; ber., small, blue.

3. J. prostrata: leaves imbricate in four rows, ovate, submucronate, glandular in the middle, appressed; berries tubercular; stem prostrate, creeping.

HAB. Gravelly shores. A shrub with very lone creeping branches; berries longer than No. 2.

592. 'TAXUS. Yew. Coniferce.

T. canadensis: leaves linear, distichous, revolute on the margin; sterile receptacle globose.

HAB. Shady rocks. April. Shrub evergreen, ?$4 \mathrm{ft}$. high; berries red.

\section{CRYPTOGA MIA.}

\section{FIIII H.}

* Capsules furnished with an articulated elastic ring. transversely opening.

593. POLYPODIUM. Sori (small clusters of capsules) roundish, scattered. Involucrum 0.

594. HYPOPELTIS. Sori roundish, having a cupshaped involicrum beneath, divided into-5-(i irregular segments. Cupsules sessile.

595. WOODSIA. Sori roundish, having an involucrum beneath, which is cut into numerous capillary segmerits. Capsules pedicellite.

596. ASPIDIUM. Sori roundh-h or elliptical, scattered. Involucrum umbilicate, or opening on one side.

597. ASPLENIU.M. Sori linear, straight, more or less transversc. Intolucrum arising from the lateral reins, and opening towards the centrit nerve or rit. 
598. SCOLOPENDRIUM. Sori linear, transverse, scattered. Involucrum double, occupying both sides of the sorus, superficial, and opening like a longitudinal suture.

599. WOODWARDIA. Sori oblong, distinct, straight, parallel with the ribs of the frond, on either side. Involucrum superficial, arched, opening inward.

600. PTERIS. Sori in a continued marginal line. Involucrum formed of the inflexed margin of the frond, opening inwards.

601. ADIANTUM. Sori oblong or roundish. Involucra membranaceous, arising from distinct portions of the frond turned in, opening inwards.

602. CHEILANTHES. Sori punctiform, distinct anct marginal, each covered with a scale-like marginal involucrum opening inwards.

603. HYMENOPHYLLUM. Sori marginal. Capsules sessile, inserted upon a common cylindrical receptacle, within a 2-valved involucrum of the same texture as the frond; valves plane, exterior free.

60\%. ONOCLEA. Sori indeterminate. Capsules covering the whole lower surface of the frond. Involucrum resembling a berry, formed of the frond, opening, but not expanding.

605. STRUTHIOPTERIS. Capsules densely covering the back of the frond. Involucrum squamiform, marginal, opening inwards.

* Capsules destitute of a ring, vascular-reticulate, pellucid, radiate at the tip and substriate, longitudinally (and often externally) opening.

606. (:SMUNDA. Capsules subglobose, pedicellate, striate, half-bivalved, paniculate. Involucrum 0.

607. SCHIZÆA. Spikes unilateral, flabellate, aggre. gate. Capsules subturbinate, striate longitudinally and radiate, opening laterally. Involucrum continuous, formed of the inflexed margin of the spikes.

308. LYGODIUM. Capsules in 2 series on the back of appendages to the frond, radiate-striate, or wrinkled, opening on the inner side, from the 
base to the summit. Involucrum scale-like, covering each capsule.

* * Capsules destitute of a ring, adnate at the base, subglobose, coriaccous, opake, not cellular, semibivalved.

609. OPHIOGLOSSUM. Capsules naked, upon an articulated distichous spike, transversely opening, 2-valved.

610. BOTRYCHIUM. Capsules subglobose, adnate to the rachis of the compound spike, distinct, naked, 1-celled; valves 2, connected behind, transversely opening.

\section{LYCOPODINEA.}

611. IXCOPODIUM. Capsules 1-celled, axillary, sessile; some of them 2-valred, filled with a farinaceous substance; others 3-valved, containing from 1-6 globose corpuscules.

\section{EQLISETACEF.}

מ12. EQUISETUM. Fructification terminal, spiked, consisting of peltate polygonous scales, on the under side of which are from $5-7$ follicular involucra opening longitudinally on the inside. sporce included in the involucrum, numerous, (green,) globose, with 4 filaments at the base which are dilated at the extremity.

\section{RIIZOCARPA.}

7313. S.ALVINIA. Involucra imbricate, $(4-9$,$) con-$ nate, resembling a unilocular capsule. Sporce inserted on a central receptacle.

614. AZOLLA. Monoicous. Sterile? appendages by pairs, contiguous. Involucrum subglobose, resembling a unilocular capsule. Sporce numerous, naked.

615. ISOETES. Capsule menbranacenus, not open. ing, immersed at the basc of the frond, 1.celled. 
- Seeds angular, attached to numerous filiform re. ceptacles.

\section{CRYPTOGAMIA.}

\section{FILICES.}

533. POLYPODIUM. Polypody.

1. P. vulgare: fronds deeply pinnatifid; segments lis near-lanceolate, obtuse, crenulate, approximate; the upper ones gradually smaller; caudex chaffy: stipe smooth.

IIAB. Shady rocks. July. Fronds 8-12 in. high, snooth; segments $3-4$ lin. broad, somexhat curved.

2. P. hexagonopterum : fronds bipinnatifid, rather smooth, circumference triangular, two of the lowe: divisions deflexed; segmerts lanceolate, obtuse, ciliate, the upper ones very entire, lower ones incisely crenate; the lowest adnate-decurrent ; sori minute, solitary; stipe smooth.

HAB. Moist wookl. Aug. Fronds $12 \mathrm{in.} \mathrm{high,}$ very broad; divisions acuminate, separate.

3. P. connectile: fronds bipinnatitid, ciliate, triangular : divisions opposite, contiguous, adnate; sergments subelliptical; stipe chaffy; sori minute.

IIAB. Woods. Aug. Fronds 12 in. high, oblong, triang. ; segments closely approximate at base.

4. P. Dryopteris : fronds ternate, bipinnate, patulous: and deflexed; segments obtuse, subcrenate; sor marsinal ; root filiform.

IIAB. Rocky woods. Aug. Frond $3-12$ in. high, triang.; stipe slend.; sori at length subconfl.

\section{HYPOPEL'TIS.}

H. obtusa.

HAB. Rocky Wroods. Aug. Fronds $8-14$ in. high, bipinnate; divisions subremote; segments oblong, the lower ones crenate-dentate; rachis pubes. ubove; sari subconfluen:. 


\section{WOODSIA.}

W. ilvensis: fronds bipinnatifid; segments oblong, obtuse, the inferior ones repand, upper very entire; hairy beneath; sori submarginal, at length confluent; stipe villous above.

HAB. Rocks. July-Aug. Fronds 6-8 in. high. beneath chaffy and hairy, cespitose.

596. $\Lambda$ SPIDIUM. Shield-fern.

* Involucrum subumbilicate or reniform, attached near. the centre of the sorus and opening on all sides.

$\dagger$ Fronds simply pinnate.

1. A. acrostichoides: fronds pinnate; segments lanceolate, falcate, acute, ciliate-serrulate, auriculate on one side at the base, subsessile, only the upper ones fructiferous; sori at length confluent ; stipe chaffy.

HAB. Shady rocks. Fronds $15-18 \mathrm{in.}$ high; seg. 1 in. long; fructiferous ones smaller.

$\dagger \uparrow$ Frond subbipinnate.

3. A. noveboracense: frond pinnate, the divisions pinnatifid, linear-lanceolate ; segments. oblong, obtuse, entire, ciliate ; sori marginal ; stipe nearly smooth.

HAB. Damp woods. July. Frond 1-2 ft. high; seg. mostly obtuse, pubes. beneath.

3. A. Thelypteris: frond pinnate; the divisions pinnatifid, linear-lanceolate, distinct at the base ; segments ovate, acute, entire; sori marginal, contiguous, at length confluent.

HAB. Wet woods and swamps. Frond a foot and a half high. Distinct from the preceding ?

4. A. cristatum: $\beta$. lancustriense: frond lanceolateovate, smooth, pinnate; divisions subcordate, pin natıfid ; segments triangular-oblong, spinulose-serrate; stipe nearly naked.

†† Frond doubly and triply pinnate.

5. A. Goldianum: frond broad-ovate, smooth, bipin$\mathrm{K}$ k 
nate; divisions lanceolate, pinnatifid; segments oblong, obtuse, with remote uncinate serratures, the lowest decurrent on the rachis; sori near the central nerve; stipe and rachis somewhat chaffy. HAB. Rocky woods. July. Frond very broad, ? $f t$. high; invol. peltate.

6. A. marginale: frond bipinnate; segments oblong; obtuse, decurrent, crenate, sinuate-repand at the base ; sori marginal ; stipe paleaceous.

HAB. Rocky places. July. Frond 18 in. high, very smooth; invol. large, umbilicate.

7. A. spinulosum : frond bipinnate; divisions oblong, decurrent, smaller seginents mucronately serrate; stipe paleaceous; involucrum glandular.

HAB. Rocky woods. July. Frond broad, slender, smooth; sori small.

8. A. dilatatum : fronds bipinnate; divisions oblong; distinct, incisely pinnatifid; segments mucronately serrate; stipe chaffy; involucrum smooth.

IIAB. Shady woods. July. Frond broad, $2 \mathrm{fl}$. high; stipe slightly chaffy.

* * Involucrum oblong, opening on one side.

9. A. asplenioides : frond bipinnate; segments lanceolate, incisely serrate; serratures 2-3-toothed; sori oblong, lunate.

HAB. Moist woods. Two ft. high; frond smooth: sori at length subconfluent.

* * Involucrum hemispherical, bursting at the top.

10. A. tenue: frond bipinnate; segments ovate, decurrent, dentate; sori solitary, near the teeth of the leaflets.

HAB. Rocks. Cespitose, 6-8 in. high, slend.; sori large; invol. deciduous.

11. A. bulbiferum: frond bipinnate, lanceolate, attenuate above; segments oblong, obtuse, serrate, the inferior ones pinnatifid; rachis bulbiferous; sori minute.

$\mathrm{H}+\mathrm{B}$. Rocky woods. Frond narrowe and long, 18 in. high; bulbs ovate, compress., green.

12. A. punctilobulum: frond bipinnate, oblong; divisions oblong, pinnatifid; segments with the supe- 
rior margin incisely dentate; stipe somewhat hairy.

LAB. Shady woods. Frond $18 \mathrm{in}$. high, thin; sori minute, near the margin.

\section{ASPLENIUM. Spleenwort.}

1. A. rhizophyllum: frond lanceolate, stipitate, subcrenate, auriculate-cordate at the base, the point very long, filiform, ronting.

HAB. Rocks. Frond $6-10 \mathrm{in}$. long, rooting at the extrcm., and producing nere plants.

3. pinnatifidum: frond pinnatifid; lobes roundishovate, the lower ones crenate.

HAB. Rocks. Laryer than the common variety.

A. A. angustifolium: frond pinvate; divisions alternate, upper ones opposite, linear-lanceolate, somewhat repand, the base truncate on the upper side, rounded on the lower.

HAB. Low wrods. July. Frond 1-2 ft. high, the fertile one separate; sori large.

3. A. ebeneum: frond pinnate; divisions lanceolate, somewhat falcate, serrate, auriculate at the base; stem smooth and polisherl.

$\mathrm{HAB}$ Rocky woods. Frond $12 \mathrm{in.} \mathrm{high;} \mathrm{stipe}$ slend., brozen; seg. $1 \mathrm{in}$. long, appearing spiral.

4. A. Trichomanes: frond pinnate; divisions roundish, crenate, obtusely cuneate, and entire at the base; stipe polished and blick.

HAB. Rocks. Cespitose $6-8$ in. high, delicate; seg. 4-5 lin. long; sori fere, large.

5. A. thelypteroides: frond pinnate; divisions pinnatifid, linceolate ; segments oblong, obtuse, serrate, contluent at the base; sori in parallel oblique lines.

HAB. Shady woods. About 2 ft. high; frond ovate; sori very numerous; stipe smouth.

ij. A. Ruta muraria : frond hipinnate at the base, simply pinnate at top; scyments cuneate, obtusely dentite at the extremity.

HAB. Dry rocks. Frond 2-3 in. high, rather co. riaceous; seg. subrhombic; stipe smooth.

7. A. montanum: frond smooth, bipinnate; divisions lanceolate, pinnatifid; segments 2-3-toothed.

HAB. Clefts of rocks on mountains. 
598. SCOLOPENDRIUM. Hart's-tongue.

S. vulgare: frond ligulate, entire, cordate at the base. HAB. Limestone rocks. Frond 8-10 in. long, rachis acute, petiolate; sori very large.

\section{WOODWARDIA.}

1. W. onocleoides: sterile frond pinnatifid; segments lanceolate, repand, slightly serrulate; fertile fronds pinnate; segments linear, entire, acute.

HAB. Sivamps. About 1 ft. high; fert. frond with very narrow seg. ; sori large, oblong.

2. W. virginica: frond very smooth, pinnate; divisions sessile, lanceolate, pinnatifid; segments oblong, obtuse, nearly entire; stipe smooth, nearly terete.

HAB. Swamps. About 2 ft. high; frond oblong; sori small, at length subconfluent.

\section{PTERIS. Brake.}

1. P. Aquilina: frond tripartite; divisions bipinnate; segments oblong-lanceolate, the lower ones pinnatifid, upper ones entire.

HAB. Woods. Tro-four ft. high; stipe long, erect, smooth; frond ample, circumf. triang.

2. P. atropurpurea: frond pinnate; inferior divisions ternate or pinnate; segments lanceolate, obtuse, obliquely truncate or subcordate at the base.

HAB. Rocks. A span high; frond grayish green; fructif. conspicuous; stipe dark br., subpubes.

3. P. caudata: frond pinnately 3-parted; sterile divisions bipinnate, with the segments linear, long, obtuse, and very entire; fertile divisions pinnate; segments rather remote, the lower ones pinnatifiddentate at the base.

HAB. Stony woods near creeks. A small species; stipe black and shining.

601. ADIANTUM. Maiden-hair.

1. pedatum : frond pedate; divisions pedate; segments rhomboid-oblong, sublunate, incisely lobed. 
HAB. Rocky woods. About 1 ft. high; stipe slend., black, polish.; frond ample; segm. entire below.

\section{CHEILANTHES.}

C. vestita: frond bipinnate, hairy on both sides; segments oblong, obtuse, very entire; stipe and rachis huiry.

HAB. Rocks. About $4 \mathrm{in}$. high, covered with chaffy broan hair.

\section{HYMENOPHYLLUM.}

H. ciliatum : frond pinnate ; lower divisions larger, upper ones gradually smaller, pinnatifid; segments linear, obtuse, bifid, ciliate, hairy on the veins ; rachis and stipe winged, ciliate.

$H \Lambda B$. Trunks of trees.

\section{ONOCLEA. Sensitive fern.}

O. sensibilis: sterile frond, pinnate ; segments lacinate, the upper ones united; fertile frond bipinnate, resembling a compound spike, with recurved globular seginents.

HAB. Low grounds. Fronds about a foot high; sterile dilated, subtriang.; fertile very narrow.

6. obtusiloba: divisions of the frond opposite, pinnatifid, with rounded lobes.

HAB. Low grounds. Sinaller than the preceding.

\section{STRUTHIOPTERIS.}

S. pennsylaraica: sterile frond bipinnatifid; segments entire, obtuse, the lowest elongated and recurved. HAB. Low grounds. About \& ft. hight; fertile frond resembl. a compound spike; seg. lin., num.

606. OSMUNDA. Flowering fern.

1. O. cinnamonea: sterile frond pinnate; divisions pinnatifid; segments ovate, oblong-obtuse, very entire; stipe woolly; fertile frond bipinate, woolly.

$\mathrm{HAB}$. Low grounds. Fronds $3-5 \mathrm{ft}$. high; stipe $\mathrm{K}$ k 2 
covered with brown hair; fertile fr. spikelike, sometimes bearing a ferv leaves.

2. O. interrupta: frond pinnate, smooth; divisions pinnatifid ; segments oblong, rather acute, entire ; some of the intermediate divisions fructiferous.

HAB. Low grounds. Frond 3-4 ft. high; several central pairs of the divisions fertile, dark-br.

3. 0 . regalis: frond bipinnate; segments oblong, distinct, serrulate; raceme terminal compound.

HAB. Swamps. About $4 \mathrm{ft}$. high, smooth; panicle large, very compound.

\section{SCHIZ无A.}

S. pusilla: frond simple, linear, compressed, tortuous ; spikes few, conglomerate at the summit of a long slender stipe.

HAB. Pine barrens. Aug. Fronds num., cespit. 2 in. long; stipe 4-5 in. long, filif.; spik. brown.

\section{LYGODIUM.}

1.. palmatum : stem flexuous and climbing; fronds conjugate, cordate, palmate, with 5 lobes; lobes entire, obtuse; spikelets oblong-linear, in a compound terminal spike.

HÂB. Shady banks of creeks. July. Frond 35 ft. high, smooth, slend. ; fr. subterm.

609. OPHIOGLOSSUM. Adder's tongue.

1. O. vulgatum: spike cauline; frond oblong-ovate, - obtuse, closely reticulate.

HAB. Wet meadows. June. About a span high; leaf solit. entire; spik. linear, 2-rowed.

2. O. bulbosum: spike cauline; frond subcordateovate, rather obtuse; root bulbous.

HAB. Low sandy grounds. About $6 \mathrm{in}$. high; frond $1 \frac{1}{2}$ in. long, 1 in. broad, reticulate.

\section{BOTRYCHIUM.}

1. B. simplex: stipe with a single frond above; frond subpinnate; segments $3-5$, rarely 7 , roundish oborate, sometimes lobed. 
HAB. Dry woods. July. About 4 in. high; frond very small, variable.

2. B. fumarioides: stipe naked; frond smooth, radical, 3-parted, bipinnate ; leaflets lunate, crenate ; spikes bipinnate.

HAB. Shady woods. Aug. A foot high; stipe rather thick; frond somezohat succul.

ß. obliquum: scape with a single frond below ; leaflets oblong-lanceolate, serrulate.

$\mathrm{HAB}$. Shady low grounds. Flowers later than the preceding.

3. B. dissectum : scape with the frond near the base ; frond tripartite-bipinnatifid; segments linear, 2. parted, 2-toothed at the extremity.

HAB. Shady woods. Aug. A span high; frond much divided; spike compound.

A. B. virginicum: somewhat hairy; scape bearing the frond in the middle; frond 3-parted, bipinnatifid; divisions incisely pinnatitid; segments obtuse, about 3-tonthed; spikes decompound.

HAB. Woods. July. Nearly $2 \mathrm{ft}$. high ; stipe mostly hairy; frond thin; spik. often geminate.

3. gracile: smooth; spike bipinnate, on a long slender peduncle.

HAB. Dry woods. A span high; frond and spike delicate, very sinooth.

\section{LYCOPODINEAE.}

611. LYCOPODIUM. Club-moss.

* Spikes pedunculate.

1. I. carolinianum: stem creeping; leaves distichous, spreading, lanceolate; perluncle erect, solitary, elongated, one-spiked; bracts sublanceolate.

HAB. Sandy swamps. Sept. Stem appressed to the ground; pedunc. 3-4 in. long.

2. L. clavatum: stem creeping, with ascending branches; leaves scattered, incurved, ending in hairs ; spikes geminate or ternate, cylindrical, pedunculate; scales ovate, acuminate, coarsely dentite.

HAB. Woods. July. Stem long, truiling; lear. ending in a diophunous bristle, entire or serrul. 
3. L. complanatum: stem trailing, with dichotowous branches; leaves 2-rowed, connate, superficial ones solitary ; peduncles elongated, supporting 4 cylindrical spikes.

HAB. Woods. Aug. Stem 2-10 ft. long; branch. repeatedly forked; leav. small, pung.

* Spikes sessile; leaves surrounding the stern.

4. L. dendroideum : erect; branches alternate, crowd. ed, dichotomous, erect; leaves linear-lanceolate. in 6 equal rows; spikes numerous, solitary, sessile.

HAB. Woods. July. About a span high; branch. numerous; spikes 3-4 on each plant.

B. obscurum: branches spreading; plant mostly 1 spiked.

HAB. Woods. Branch. diverging, loose; spike as in the preceding.

5. L. annotinum: stem creeping; branches ascending, dichotomously branched; branchlets simple; leaves in 5 rows, linear-lanceolate, mucronate, serrulate, spreadıng; spikes oblong-cylindrical, solitary, sessile, terminal.

HAB. Rocky and mountain woods. Aug. dbout a span high; branch. $4--8$; spik. 1 in. long.

n. L. inundatum: stem creeping; branches simple, solitary, erect, with a single sessile leafy spike at the extremity ; leaves linear, scattered, acute, entire, curved upwards.

HAB. Low wet grounds. July. Stem 3-4 in. long; flowering branch. subradical.

7. L. selaginoides: stem creepinir ; branches ascending, simple; leaves scattered, lanceolate, somewhat spreading, ciliate-denticulate; spikes terminal, solitary.

HAB. Mossy woods. July.

3. L. alopecuroides: stem creeping, somewhat branched; liranches nearly simple, elongated, ascending, with a sessile leafy spike at the summit; leaves linear-subulate, ciliate-dentate at the base, spreading.

$\mathrm{HAB}$. Sandy and sphagnous swamps. Oct. Stem 
long; branches densely leaved, terete, 8-12 in. high.

9. L. rupestre: stem creeping, with ascending subdivided branches; leaves scattered, imbricate, linear-lanceolate, ciliate, ending in hairs; spikes solitary, sessile, quadrangular.

HAB. Rocks. July. Stem 2-3 in. long; branch. numerous, somewhat incurved; plant grayish-green.

** Spikes sessile; leaves distichous.

10. L. apodum: leaves 2-rowed, roundish-ovate, acute, denticulate, flat, acuminate; spikes terminal, sessile, nearly solitary, quadrangular.

HAB. Wet rocky places. July. Resembling a a moss, or Jungermannia ; creeping.

**** Fructification axillary.

11. L. lucidulum: leaves in 8 rows, linear-lanceolate, denticulate, acute, spreading-reflexed; stem ascending, bifid.

HAB. Woods. July. Stem 3-12 in. long, nearly erect, simp. or bifid, dark green.

12. L. Selago: stem dichotomously branched, erect, fastigiate ; leaves scattered in 6 equal rows, linearlanceolate, acuminate, entire, imbricate, rigid; fruit axillary.

HAB. High mountains. About 6 in. high; lear. densely imbricated.

\section{EQUISETACEA:}

\section{EQUISETUM. Horse-tail.}

1. E. hyemale: stem simple, erect, very rough, naked; sheaths whitish, black at the base and sum. mit.

HAB. Woods and marshes. April. Stem $2-3 f t$. high, slend., pale grecn; spik.term., mostly solitary.

2. E. arvense: sterile stems decumbent, with simple branches, which are rough and quadrangular; fertile stem erect, simple; sheaths simple, cylindrical, incisely-dentate. 
HAB. Boggy grounds. April. A span high; sheaths of the fertile stems swelling; teeth black.

3. E. sylvaticum: sterile and fertile stems with compound scabrous deflexed branches.

HAB. Woods and low grounds. May. Branch. verticill., curved downwards; sheaths loose.

4. E. limosum: stems branched upwards, with the branches about 12 in a whorl, simple, pentagonal, smooth.

HAB. Borders of ponds, \&c. July. Stems 2-4 ft. high; sheaths with narrow acute black teeth.

5. E. variegatum: cespitose ; stems somewhat branched at the base, naked, scabrous, filiform; sheaths 3 -toothed, blackish ; teeth membranaceous, lanceolate, whitish, deciduous at the tips.

$\mathrm{H} \Lambda \mathrm{B}$. Borders of lakes. Aug. Stem 3-6 in. long, 5-angled; rough with siliceous dots.

\section{RHIZOCARPA.}

\section{SALVINIA.}

S. natans: leaves ovate, subcordate, obtuse, with fascicles of hairs beneath, scabrous.

HAB. Lakes and still waters. Floating.

\section{AZULLA.}

A. caroliniana: leaves distichous, imbricate, ovateoblong, obtuse, spreading.

HAB. Lakes and still waters. Floating; resembling: a Jungermannia; leav. reddish beneath.

\section{ISOETES.}

1. lacustris.

$\mathrm{HAB}$. Bottom of lakes. Leav. all radical, $2-5 \mathrm{in}$. long, subulate, fleshy, 'senicylindrical. 
[ OMITTED.]

435. FUMARIA. Fumitory. Fumariacex.

E. officinalis : silicles globose-retuse : fructiferous pedicels erect, twice as long as the bract; racemes rather lonse ; stems erect ; leaves decompound, with linear lobes.

HAB. Sandy field!s. ๑. July-Aug. Fl. purp. §. 



\section{INDEX OF THE GENERA.}

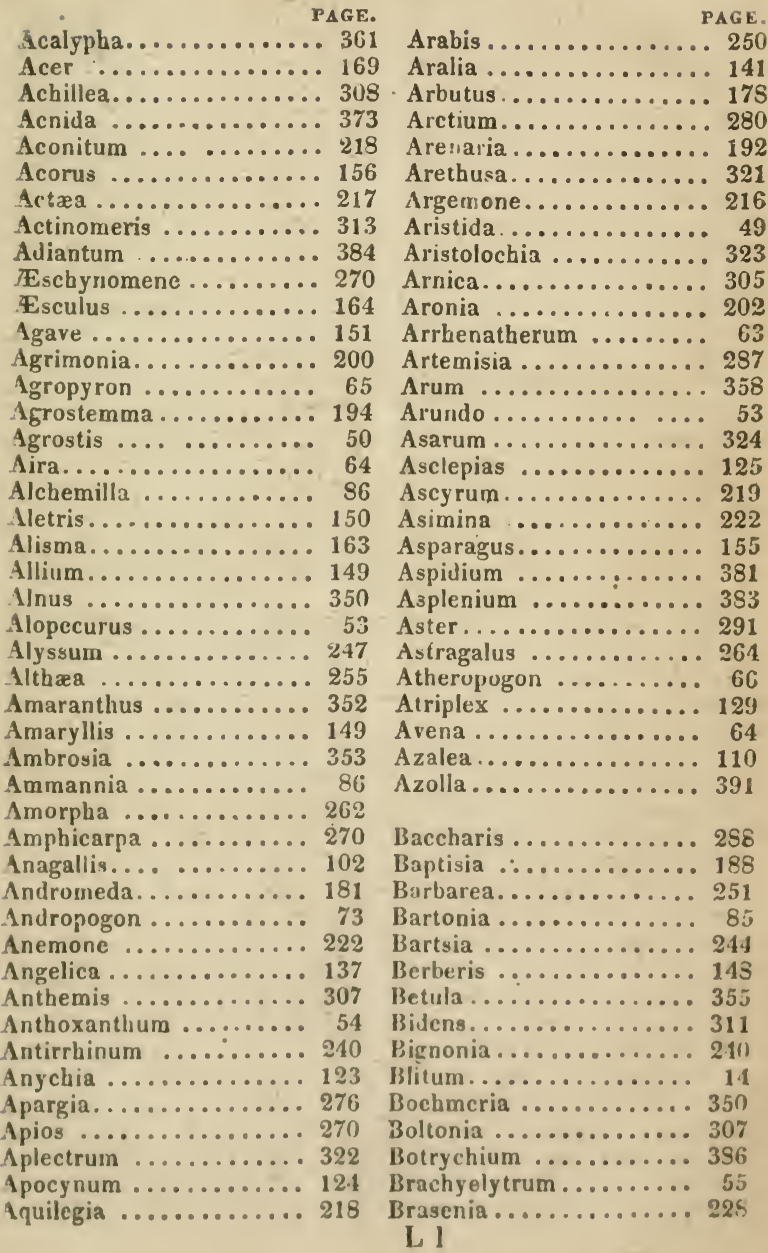


Briza.............. 56

Bromus .............. 63

Buchnera ...........240

Cacalia ............. 286

Cactus............... 198

Cakile............. 246

Calla ............... 358

Callitriche........... 14

Calopogon ........... 321

Caltbs ..............224

Calycanthus .......... 211

Camelina ............ 248

Campanula............ 111

Cardamine ........... 248

Carex .............. 333

Carpinus............. 356

Carya ................ 357

Cassia............... 187

Castanea............. 355

Catalpa.............. 20

Caulinia ............. 330

Ceanothus ........... 119

Celastrus ............. 120

Celtis .............. 132

Cenchrus ............. 46

Cephalanthus .......... 78

Cerastium............ 193

Ceratochloa.......... 62

Ceratophyllum ......... 354

Cercis ................ 188

Chærophyllum ........ 354

Cheilanthes............ 385

Chelone ............243

Chelidonium.......... 216

Chenopodium......... 130

Chionanthus.......... 17

Chrysocoma.......... 286

Chrysosplenium ........ 189

Chrysanthemum....... 307

Cichorium............ 276

Cicuta ............. 135

Cimicifuga .......... 219

Cinna ............. 52

Circæa............. 24

Cissus ............. 121

Claytonia ........... 119

Clematis ............ 222

Cleome .............. 252

Clethra ............ 187

Clinopodium.......... 236

Clitoria...........2 271

Cnicus............. 281
Cnidium ............ 134

Collinsia ............241

Commelina ........... 35

Comptonia ............ 349

Conium............. 136

Conostylis .......... 150

Convallaria........... 153

Convolvulus ........... 107

Conyza............28 288

Coptis ..............225

Corallorhiza.......... 322

Coreopsis ........... 312

Cornus ........... 82

Corydalis .......... 259

Corylus............. 360

Cratxgus ............ 201

Crotalaria............ 262

Crotonopsis .......... 352

Crypsis............ 53

Crypta............. 24

Cucubalus........... 190

Cunila.............. 22

Cuphea............20 200

Cupressus............ 361

Cuscuta ............. 128

Cynodon ........... 54

Cynoglossum ......... 102

Cyperus ............ 42

Cypripedium.......... 322

Dactylis .......... 62

Dalibarda............ 208

Danthonia........... 62

Datura ........... 110

Daucus ............. 135

Delphinium .......... 218

Dentaria ............248

Dianthus ........... 190

Diapensia.......... 110

Diervilla ........... 113

Digitaria ............ 72

Diarrhena........... 62

Diodia............. 80

Dioscorea............ 374

Diospyros........... 375

Dipsacus ........... 78

Dirca............. 170

Dodecatheon ......... 104

Dolichos ...............

Draba .............247

Dracocephalum ........236

Drosera ............. 142

Dryas.............208 
Dulichium........... 42

Echium

101

Elephantopus .......... 314

Eleusine ............. 65

Elodea ............ 376

Elymus............. 66

Empetrum ........... 372

Epiphagus ............ 244

Epizæa ............ 184

Epilohium ............ 168

Equisetum .......... 389

Erigeron ............ 289

Eriocaulon .......... 350

Eriophorum ......... 45

Ervum ............264

Eryngium .......... 132

Erythronium .......... 152

Euchroma...........243

Eupatorium ......... 283

Euphorbia........... 331

Evonymus ........... 119

Exacum ............ 85

Fagus .............. 360

Fedia.............. 34

Festuca............. 60

Floerkea........... 149

Fragaria............ 211

Frasera............. $\$ 5$

Fraxinus ............ 371

Fuirena............ 45

Fumaria ............ -

Galactia ............271

Galcopsis ............ 234

Galium ............ 78

Gaultheria........... 179

Gaura............... 163

Genista ........... 262

Gentiana ........... 127

Geranium ........... 254

Gerardia ............241

Geum .............209

Gillenia...........203

Glaux ............. 124

Glechoma ............234

Gleditschia ........... 375

Glyceria .......... 5.5

Graphalium ............ 2 $2^{24}$

Gonolobus ............ 125

Goodyera........... 319

fratiola!.............
Gymnocladus......... $37 €$

Gymnoprogon .......... 54

Gyromia............ 161

Habenaria ............ 317

Hamamelis .......... S6

Hamiltonia ............ 123

Hedeoma........... 22

Hedyotis ........... 81

Hedysarum .......... 267

Helenium ............ 308

Helianthemum ........ 215

Helianthus ............ 308

Heliopsis ........... 308

Helonias ........... 159

Hemerocallis ......... 151

H-mianthus.......... 20

Hepatica ........... 225

Heracleum ........... 137

Herpestis ........... 239

Hesperis ............281

Heteranthera ......... 36

Heuchera ........... 129

Hibiscus ............ 255

Hieracium .......... 279

Hierochloa .......... 77

Hippuris ............ 13

Hoicus............ 71

Hinlosteum .......... 74

Hordeum ........... 73

Hottonia ............ 104

Houstonia .......... 81

Hudsonia ............ 215

Hunulus ............ 373

Hydrangea ........... 18s

Hidrastis . .......... 224

Hydrocbloa .......... 354

Hydrocotyle.......... 133

Hyilrophyllum ........ 105

Hymenophyllum ....... 385

IIyoscramus........... 107

Hvperirum ........... 219

Hypoxis ............ 150

Hypopeltis ........... 380

Hyssojus ........... 233

J. ffersonia ......... 170

llex .............. 87

Impatiens............ 115

Inula ............. 290

Iponioca............. $10 £$

Iris $. . . \ldots \ldots \ldots \ldots . . . . .35$

19 Isanthus .......... 231 
Isnardia ..............

Isoetes

83 Lygodium........... 38b

Itea

Juglans

Juncus 357

Juniperus

156

Justicia

376

Iva.

Kalmis

Koleria. 182

Krigia

Kubnis

280

Kyllingia .......... 37

Lachnanthes ............

Lactuca................

Lamium .............

Lathyrus ..............

Laurus ............. 174

Lechea ..............

Lecontia ..............

Ledum ........... 186

Icersia............. 47

Leiophyllum.......... 187

Lemna ............ 24

Leontice............ 148

Leuntodon ........... 277

Leonurus ........... 23i

Lepidium ............248

Leptandra............ 19

Lespedeza ........... 266

Liatris . . . . . . . . . . . 282

Ligusticum ........... 156

Ligustrum........... 17

Lilium ............ 152

Limosella ............ 239

Lindernia ........... 19

Linnæa............ 82

Linum ............ 142

Liquidlambar.......... 357

Liriodendron.......... 221

Listeria............. 320

Lithospermum ......... 100.

Lobelia............. 112

Loliun ............. 64

Lonicera ............ 113

Ludwigia............ \$ \$3

Lupınus ............. 252

Luzula ............. 158

Lycopodium .......... 387

Lyenpsis ............. 102

I.ycopus ..............

Lysimachia ........... 102

Lythrum ............200

Magnolia ...........221

Malaxis ............. 321

Malva.............255

Mariscus............ 42

Marrubium ..........235

Martynı..........2 243

Meconopsis .........216

Medicago...........271

Melamipyrum .........2 244

Melanthium .......... 159

Melica ............. 60

Melilotus ............ 264

Melothria............ 361

Menispermum ........ 376

Mentha............. 233

Menyanthes.......... 104

Menziesia........... 169

Mikania .............286

Milium ............. 45

Mimulus ........... 242

Mitchella.......... 81

Mitella ............ 189

Mollugo ........... 74

Momordica........... 361

Monarda............ 22

Monotropa ........... 185

Mnrus .............. 352

Muhlenbergia......... 49

Mvosotis ........... 101

Myrica ............. 372

Myriophyllum ......... 354

Myrrbis ............ 135

Nasturtium ..........251

Naribecium .......... 151

Nelumbium .......... 228

Neottia............ 320

Nepeta...........233

Nicotiana ........... 107

Nuphar .............217

Nymptæa........... 218

Nyssa ...........272

Obolaria ............. 85

Enanthe ..........

Enothera........... 166

Onopordum ..........280

Onoclea ............. 385

Onosmodium ......... 10 1 


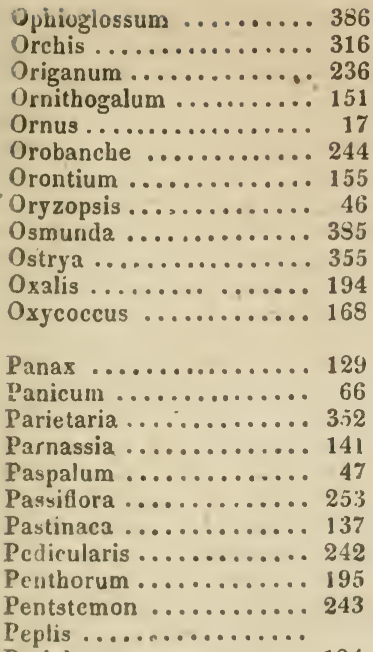

Periploca ........... 124

Phacclia .......... 106

Phalangium ......... 151

Phalaris........... 54

Phaseolus ........... 270

Phleum............. 54

Phlox ............... 103

Phryma............ 238

Phyllanthus ......... 36 l

Phragmites .......... 55

Pliysalis............110

Phytolacca ........... 196

Pinus .............. 359

Piptatherum ......... 48

Pisum .............263

Plantago ............ 84

Platanus ............ 355

Poa .............. 56

Podophyllum .........2 217

Podostcmum .......... 332

Poronia ............ 320

Polanisia ........... 2.52

Polemonium.......... 109

Polyzala ............2 260

Polygonum .......... 171

Polymnia ........... 314

Polypodium .......... 330

Polypogon ........... 52

Pontederia ......... 150
Populus............ 375

Portulacca........... 216

Potamogeton ......... $8 s$

Potentilla........... 209

Prenauthes ...........277

Primula............. 103

Prinos .............. 148

Proserpinaca......... 74

Frunella............ 236

Prunus ............. 198

Psamma............. 53

Pielea............. 86

Pteris .............. 384

Pterospora ............ 185

Pulmonaria .......... 100

Pycnanthemum .......235

Pyrula............ 185

Pyrus..............203

Quercus............. 357

Ranunculus .........225

Rapisanus ...........252

Rhamnus ........... 120

Rhexı............ 165

Rhododendron ........ 183

Rhodora ........... 184

Rhus ............ 140

Rhynchospora ........ 41

Ribes.............. 121

Rivina............ 86

Robinia...........271

Rochelia ............ 102

Rosa.............. 205

liubus .............206

Ruellia ............240

Rudbeckia ........... 310

Rumex ............ 162

Ruppia........... 89

Sabbatia .......... 105

Sagina ........... 87

Sayittaria........... 355

Salicornia ........... 13

Salix ............. 365

Salsola ........... 131

Salvia ............. 23

Salvinia ........... 390

Sambucus ............ 139

Samolus ............. 104

S.ınuinaria ......... 217

Sanguisorba .......... 82

Sanicula ............ 13.3 L. 12 
Saponaria ........... 189

Sarracenia .......... 217

Saururus ............ 163

Saxifraga ........... 188

Scheuchzeria ......... 160

Schizæa ............ 386

Schœnus ............ 41

Schollera ........... 36

Scirpus ........... 37

Schwalbea ........... 243

Scleria............. 349

Scleranthus .......... 190

Scrophularia.......... 239

Scutellaria ............ 236

Secale ............ 6j

Sedum ............. 195

Senecio............. 305

Sesuvium .......... 202

Setaria ........... 72

Shepherdia ........... 376

Sibbaldia ............. 142

Sicyos ............. 362

Sida ............. 254

Silene ............ 190

Silphium .......... 313

Sinapis ........... 252

Sison .............. 134

Sisymbrium ........ 251

Sisyrinchium ......... 36

Sium ............. 136

Smilax ........... 373

Smyrnium .......... 134

Solanum ........... 111

Solidago ........... 300

Sonchus ............278

Sorbus .......... 202

Sparganium ......... 333

Sparganophorus ........ 287

Spartina............. 46

Spergula ............ 193

Spermacace .......... EU

Spigelia ............ 106

Spiræa ............. 203

Stachys............ 23t

Staphylea ........... 141

Statice ............ 142

Stellaria ............ 191

Stipa .............. 48

Streptopus ........... 153

Strophostyles .........270

Struthiopterus......... 385

Stylosanthes .......... 266

Subularia ........... 248
Swertia........... 85

Symphoria .......... 114

S5mplocarpus........ 86

Talinum ...........216

Tanacetum .......... 287

Taxus .............. 377

Tephrosia ............271

Teucrium ........... 232

Thalictrum ........... 223

Thapsia ............. 138

Thesium ............ 123

Thlaspi............. 246

Thuya .............. 361

Tiarella .......... 189

Tilia ............. 214

Tilıæa............ 8 7

Tipularia ............ 321

Tofieldia ........... 160

Tradescantia.......... 147

Trichochloa.......... 52

Trichodium .......... 49

Trichostema.......... 238

Tricuspis .......... 60

Trientalis ............ 164

Trifolium ........... 265

Triglochin .......... 160

Trillium ........... 161

Triosteum ..........115

Triphora .......... 321

Tripsacum ........... 349

Trisetum........... 62

Triticum ........... 65

Trollius............. 225

Tus:ilago ............ 30

Typha .............. 333

Ulmus ............. 132

Uniola ............ 55

Cralepis............. 69

Urtica ............. 351

Utricularia ........... 29

Uvularia ............ 153

Vaccinium ........179

Valisneria ............ 365

Veratrum ........... 159

Verbascum ........... 106

Verbına............23s

Verbesina ........... 307

Vernonia ........... 283

Veronica ........... 17

Yiburaum ............ 13 
Vicia .............263 Xerophyllum ......... 160

Villarsia ............ 104 Xylosteum ........... 114

Viola .............. 115 Xyris .............. ${ }_{36}$

Viscum .............. 372

Vitis ............ 120 Zannichellia ......... 330

Zanthorhiza........... 143

Woodsia............ 381 Zanthotylum .......... 373

Woodwardia ......... 394 Zapania ............. 239

Zizania................. 350

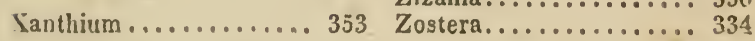




\section{N D E X}

OF THE

PRINCIPAL SYNONYMS.

- Acer striatum. du Roi. See A. pennsylvanicum.

Actcea brachypetala. D. C. racemosa. $\mathrm{L}$.

Agrostis cinna. L.

Cinsicifuga racemosa.

Cinna arundinacea.

diffusa. Muhl.

- filiformis. Muhl.

A. sylvatica.

A. lateriflora. $\beta$.

_ - glauca. Muhl.

Arundo coarctata.

Aira mollis. Muhl.

- pennsylva:ica. Muhl.

- truncata. Mubl.

A. lateriflora.

Koeleria pennsylv.

K. truncata.

Alopecurus aristul. Mx.

Ammi capillaceum. $\mathrm{Mx}$.

A. geniculatus.

Sison capillaceus.

. Ampelopsis quinquef. $\mathrm{Mx}$.

Ammyrsine buxifol. Ph.

Cissus hederacea.

Andromeda paniculata. Walt.

Leiophyllum buxif.

- paniculat. Willd.

- Indropogon ambigium. Mx. - purpurasc. Mubl.

Anemone thalictroides. L. hepatica. L.

A. racemosa.

A. ligustrina.

Gsmnopogon.

A. scoparium.

Thalictrum anemonoid.

Hepatica triloba.

Inthopogon lepturoides. N. Arenaria marina. Sm.

- canadensis. Pers.

Arethusa ophioglossoid, \&c.

- pendula. Sw.

Gymnopogon.

\}

A. rubra. $\beta$.

Pogonia ophiogloss.

Triphora pendula.

Aristida racemosa. Muhl. - Ironia melanocarpa. Ell. - Arum virginicum. L. Arundo agrostoides. Ph.

- arenaria. L. Pliragmites L.

.1sclepias obovata. Ell. - lancifolia. Ives. .9spidium obtusum. Willd. filix mas. $\mathrm{Ph}$.

. Asplenium melanocoulon. IV. Alragene americana. Sims. Avena elatior. L.

- pennsylvanica. Muhl.

. Azalen nudiflora, \&c.

A. purpurascens.

A. arbutifol. $\beta$.

Lecontia virginica.

A. canadensis.

Psamina arenaria.

Pliragmites.

A. viridiflora. $\varangle$.

Hypopeltis obtusa.

A. Goldianum.

A. trichomanes.

Clematis verticillaris. Arrhenatherum avenen. Arr. pennsylvanicum. Rliododendron. 
Bartsia coccinea. L. See Euchroma coccinea.

Batschia canescens. Mx.

Bootia sylvestris. Big.

Briza canadensis. Mx.

Callitriche aquatica. Big. Caprifolium dioicum, \&c. Caulophyllum thalictr. Mx. Centaurella panicul. Mx. Coutumnale. $\mathrm{Ph}$. Cerastium glutinosum. N. Chaerophyll. canad. Pers. Claytoni. Pers.

Chelidonium diphyll. $\mathrm{Mx}$. Chinaphila maculat., \&c. Chironia angularis, \&c. Cistus canadensis. L. Cleome dodecandra. $\mathrm{Pb}$. Cormardra umbellatu. N. Comarum palustre. L. Coreopsis alternifolia. L. Convolvulus stans. Mx. Conyza marilandica. Mx. Cuxila pulegioides. L. Cyamus luteus. Salisb. Cymbidium pulchell. IV. odontorrhizon, \&c. hyemale. W.

Cynogloss, amplexic. Mx. Cyperus cespitosus. Tor. parviflorus. Muhl. uncinatus. $\mathrm{Ph}$.

Dicksonia pilosius. WV. Digitaria passpalod. MIx. Dilutris tinctoria. Ph. Dioscorea glauca. Mubl.

Elod a virginica. $\mathrm{Pl}$. Epipactis convallartoid, \&c. Equisetum scirpoides. Mx. Eriophorum cespitostem. $\mathrm{Pb}$. Euphorbia depres:a. Tor. Erysimum officinale. L.

Festuca diundra. Mx.

- fluitans. L.

— polystachia. Mx. - unioloides. Willd.

Lithospermum canesc. Potentilla coarctata. Poa canadensis.

C. verna. Lonicera. Leontice thalictroid.

\}

Bartonia.

C. nutans.

Myrrhis canadensis.

M. Claytoni.

Meconopsis dipbyll.

Pyrola.

Sabbatia.

Helianthemum.

Polanisia graveolens.

Thesium umbellat.

Potentilla.

Actinomeris squarrosa.

C. spithamæus.

C. camphorata.

Hedeona pulegioides.

Nelumbium.

Calopogon.

Corallorhiza.

Aplectrum.

C virginicum.

C. Nuttallii.

C. dentatus.

C. inflexus.

Aspid. punctilobulum.

D. glabra.

Lachnanthes.

D. quaternata.

Hypericum virginicum Listeria.

F. variegatum.

E. vaginatum.

E. inarulata.

Sisymbrium officinale

Diarrhena americana.

Glvceria.

F. fascicularis.

Ceratochloa.

Galium strictum. Ed. $\left.\begin{array}{l}\text { Galium strictum. Ed. } \\ \text { Geptentrionale, R. \&: S. }\end{array}\right\}$ Gratiola neglecta. Tor.

G. boreale.

G. quercifolia

G. virginica. 
Glycine monoica, L. See Amphicarpa.

helvolus. Muhl. Strophostyles.

Heritiera tinctoria. Bosc. Lachnanthes.

Heteranthera gramin. Wahl. Schollera.

Hippophäe canadens. W. Shepherdia.

Holcus fragrans. W. Hierochloa.

Hydropeltis purpurea. Mx. Brasenia.

Hypericum coryinbos. $W$. H. punctatum.

Ictodes foetida. Big.

Iris gracilis. Big.

- prismatica. $\mathrm{Ph}$.

$\left\{\begin{array}{l}\text { Symplocarpus. } \\ \text { I. virginica. }\end{array}\right.$

Jugluns alba, \&c.

Juncus campestris, \&c.

Juniperus repens. $\mathbf{N}$.

Lathyrus maritimus. Big.

Ledum buxifolium. Ait.

Limnetis juncea, \&c.

Lespedeza polystachya. Mx.

- erpitata. Mx.

Lobelia paludosa. Nut.

- gracilis. $\mathbf{N}$.

Medeola virginica. L.

Micropetalum gramin. Mx.

- lanceolatum. Mx.

Nymphoea advena, \&c.

Neottia pubescens, \&c.

Oldenlandia glomerat. Mx.

Orobanche americana. W.

Orchis ciliaris, \&c.

- discolor. Ph.

Oryzopsis melanocarpa. Muhl.

Panicum viride, \&c.

Pedicularis serotina. Mubl.

Peplis americana. Ph.

Phaseolus trilobus. Vix.

Poa quinquefida. $\mathrm{Ph}$.

- - seslerioides. Mx.

Polypodium calcareum. W.

Porcelia triloba.

Potentilla floribunda. $\mathrm{Ph}$.

Pulnonaris maritina. L.

Pyrola chlorantha. N.

Pyrus arbutifolia, \&c.

Queriu canadensis. L.

Carya.

Luzula.

J. prostrata.

Pisum maritim.

Leiophyllum.

Spartina.

L. hirta.

L. frutescens.

L. Durtmanna.

L. Nuttallii.

Gyromia.

Stellaria longifol. lanceolatum.

Nuphar.

Goodyera.

Hedyotis.

Epiphagus.

Habenaria.

Tipularia.

Piptatherum nigrum.

Setaria.

P. pallida.

Crypta minima.

Strophostyles angulosa.

\} Tricuspis.

P. Dryopteris.

Asimina triloba.

P. fruticosa.

Lithospermum marit

$P$ asarifolia.

Aronia.

Anychia dichotoma. 
R3hynchospora longirost. Mx. See R. laxa.

Rosa suaveolens. $\mathrm{Ph}$.

R. Rubiginosa.

Rubus procumbens. Muhl.

R. trivialis.

Salicornia virginica. L.

Sarothra gentianoid. L.

Schoenus albus, \&c.

Scirpus cyperiform. Muhl.

- robustus. $\mathrm{Ph}$. trichodes. Muhl.

Selinum canadense. Mx.

Serpicula verticill. Muhl.

Sisymbrium officinale.

Sium rigidius. L.

ambiguum. N.

Smilaeina borealis, \&c.

Smyrnium atropurp. Lam. trifoliatum. N. aureum. L.

Smyrnium integrifol. L.

Spartina polystachya. M.

Spergulastrum. Mx.

Spircea stipulacea, \&c.

Stipa sericea. Mx.

Stylophorum diphyll. Nutt.

Sivertia corniculata. $\mathrm{Ph}$.

Trichophorum cyperin. Pers.

Trientalis Europea. Mx.

Trillium pictum. $\mathrm{Ph}$.

Triticum repens, \&ic.

Thlaspi Bursa pastor. L.

Troximon virginicum. Pcrs.

Turritis hirsuta, \&c.

Udora canadensis. Nutt.

Uniola distichophyll.

Utricularia ceralophyl. Mx.

Uvularia amplexif., \&cc.

Veratrum luteum. L.

Veronica virginica. L.

Viburnum opulus. Mx.

Vicia pusilla. Willd.

- Mitchilli. Raf.

Viola bicolor. Ph.

- debilis. $\mathrm{Pb}$.

- slriata. Ait.

Windsoria poceformis.

__ pallida. Spr.

Xanthium maculatum. Liaf. orientale.

Zizania fluilans. Mx.

S. herhacea.

Hypericum Sarothra.

Rhynchospora.

Cyperus mariscoid.

S. macrostachyos.

S. acicularis.

Cnidium canadensis.

Elodea.

Nasturtium.

Pastinaca rigida.

Convallaria.

Cnidium atropurp.

S. cordatum.

Sison aureus.

Sison integrifolius.

S. cynosuroides.

Stellaria.

Gillenia.

Trichochloa capillaris.

Meconopsis.

S. deflexa.

Scirpus erioph.

T. americana.

$T$. erythrocarpum.

Agropyron.

Krigia amplex.

Arabis.

Elodea canadensis.

U. spicata.

U. inflata.

Streptopus.

Helonias dioica.

Leptandra.

V. oxyeoccus.

Ervum tetrasperm:

- hirsutum.

V. tenella.

V. Muhlenbergii.

V. ochroleuca.

Tricuspis.

Poa dentata.

X. macrocarpon.

Ifydrochloa. 

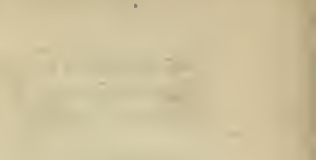




$$
-
$$





\section{QK117 .T6 C.2 \\ Torrey, John/A compendium of the flora 0 \\ || || || || || || | || || [|| | || || || || || || || || || || || \\ || ||||||||||||||||||||||||||||||||||||||||||||||||||||||||||| \\ 35185001250925}




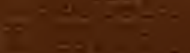

